\title{
Availability of Water in
}

\section{Kalamazoo County,}

\section{Southwestern Michigan}

GEOLOGICAL SURVEY WATER-SUPPLY PAPER 1973

Prepared in cooperation with Kalamazoo County and the State of Michigan

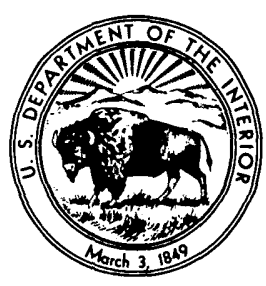





\section{Availability of Water in}

\section{Kalamazoo County,}

\section{Southwestern Michigan}

GEOLOGICAL SURVEY WATER-SUPPLY PAPER 1973

Prepared in cooperation with Kalamazoo County and the State of Michigan

A study designed to provide water facts for planning orderly development and guiding management of water resources of the county, with special reference to quantitative appraisal of ground-water reservoirs 
UNITED STATES DEPARTMENT OF THE INTERIOR

ROGERS C. B. MORTON, Secretary

\section{GEOLOGICAL SURVEY}

W. A. Radlinski, Acting Director

Library of Congress catalog-card No. 78-180654

For sale by the Superintendent of Documents, U.S. Government Printing Office Washington, D.C. 20402 (paper cover) Stock Number 2401-1140

$$
\text { क } 6.25
$$




\section{CONTENTS}

\begin{tabular}{|c|c|}
\hline & Page \\
\hline - & 1 \\
\hline roduction............ & 2 \\
\hline Purpose and scope & 3 \\
\hline Acknowledgments and personnel & 3 \\
\hline Location & 4 \\
\hline Topography & 4 \\
\hline Climate & 5 \\
\hline drologic system & 6 \\
\hline Surface-water system & 6 \\
\hline Ground-water system & 7 \\
\hline Physical characteristics of aquifers & 8 \\
\hline Hydraulic properties of aquifers & 9 \\
\hline Ground-water-surface-water relationships & 11 \\
\hline Recharge from precipitation and the hydrologic budget. $\ldots . .$. & 12 \\
\hline Natural discharge and movement of ground water & 18 \\
\hline Chemical and physical quality of water & 21 \\
\hline Temperature of ground water & 21 \\
\hline Water quality in relation to use & 26 \\
\hline Public and domestic supplies & 26 \\
\hline Industrial supplies. & 29 \\
\hline Agricultural supplies. & 31 \\
\hline ilability of water & 31 \\
\hline Streams & 31 \\
\hline Flow duration & 33 \\
\hline Low-flow frequency & 34 \\
\hline Storage analysis & 40 \\
\hline Floods & 44 \\
\hline Chemical quality & 46 \\
\hline Withdrawals & 48 \\
\hline Lakes. & 54 \\
\hline Austin Lake complex & 55 \\
\hline Water budget in the Austin Lake complex & 59 \\
\hline Ground-water augmentation to Crooked and Eagle Lakes. . . . . & 63 \\
\hline Lakes and reservoirs as a source of water supply & 66 \\
\hline Chemical quality & 71 \\
\hline Austin and West Lakes & 73 \\
\hline Long Lake.... & 73 \\
\hline Sugarloaf and Gourdneck Lakes & 73 \\
\hline Eagle and Crooked Lakes & 75 \\
\hline Gull Lake. & 75 \\
\hline Sherman Lake & 76 \\
\hline Barton Lake & 76 \\
\hline Indian Lake. & 77 \\
\hline
\end{tabular}


Availability of water-Continued $\quad$ Page

Ground-water reservoirs

Kalamazoo River ground-water reservoir

Idealized reservoir................................. 81

Water potentially available to the reservoir

Practical development of existing pumping centers._._. $\quad 85$

Development of the reservoir.

Chemical quality .................. 87

Schoolcraft ground-water reservoir._.

Idealized reservoir.

Water potentially available to the reservoir............ 88

Future development of the reservoir.

Kalamazoo-Portage ground-water reservoir _........... 91

Idealized reservoir................................ 92

Water potentially available to the reservoir......... 92

Practical development of existing pumping centers......... 93

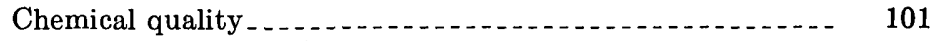

Other ground-water reservoirs......... 102

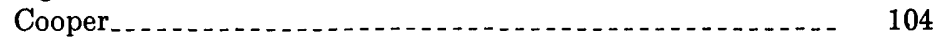

Morrow Lake. ......... 105

Augusta-Galesburg- . 106

Alamo-Oshtemo .

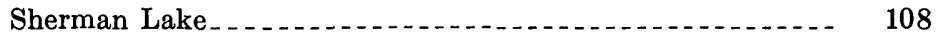

Vicksburg $\ldots$

Texas_._.

Isolated well field...

Summary of potential development.

Nonreservoir areas._._.

Ground-water withdrawals. . .

Legal considerations

Summary and conclusions.

Selected references......

Index

\section{ILLUSTRATIONS}

\section{[Plates are in pocket]}

Plate 1. Map and hydrologic sections showing saturated thickness of upper and lower aquifers in Kalamazoo County, southwestern Michigan.

2. Map showing transmissibility of upper and lower aquifers.

3. Hydrographs showing separation of total runoff into components of ground- and surface-water runoff, water levels, and precipitation, Kalamazoo River and St. Joseph River basins.

4-8. Maps showing:

4. Generalized water-table contours, low-flow discharge of streams, and saturated thickness of upper aquifer.

5. Streamflow. 
Plates 4-8. Maps showing-Continued

6. Chemical quality of surface water and relation of dissolved solids to discharge.

7. Water-level contours for the Austin Lake complex, November 1964 and May 1966.

8. Distribution of ground-water pumpage in 1966 and chemical quality of ground water.

9. Mathematical models of Cooper, Morrow Lake, AugustaGalesburg, Alamo-Oshtemo, Sherman Lake, Vicksburg, and Texas ground-water reservoirs showing assumed pumping wells and array of imaginary wells.

Figure 1. Index map and map of Kalamazoo County showing surface-water system..................................

2. Graph.showing cumulative monthly precipitation departure from long-term monthly norms

3. Map showing distribution of annual precipitation, 196366.

4-7. Graphs showing:

4. Relationship of total runoff to ground-water recharge in Kalamazoo River basin, 1948-62...-

5. Thermographs of selected wells in 1965 and 1966 .

6. Relationship between annual variations in groundwater temperature and depth of well

7. Relationship between depth of well and arrival time of surface heat. .......

8. Records of streamflow data

9-14. Graphs showing:

9. Flow-duration curves of daily discharge for selected gaging stations in 1965 and $1966 \ldots \ldots$....

10. Low-flow frequency curves for Kalamazoo River at Comstock . . . . . . .

11. Frequency-mass curve and draft-storage lines for 20-year recurrence interval, Kalamazoo River at Comstock . . . . . .

12. Regional draft-storage curves for 20-year recurrence interval for southwestern Michigan...............

13. Regional draft-storage curves for 10 -year recurrence interval for southwestern Michigan ...............

14. Flood-frequency curve, Kalamazoo River at Comstock, 1931-67

15. Map showing flood-frequency regions

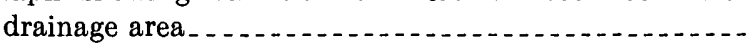

17. Graph showing frequency of annual floods, flood-frequency regions $\mathrm{C}$ and $\mathrm{E}$

18. Comparison of surface-water withdrawal in 1966 with dependable supply

19. Lake hydrographs showing improvement of water levels due to diversion of water from Sugarloaf Lake outlet

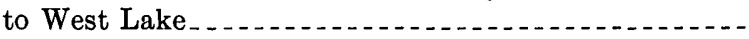

20. Photographs showing effects of drought in 1963 and 1964 
Figures 21-23. Graphs showing:

Page

21. Effects of pumping from deep aquifer on levels of Bass, Crooked, and Eagle Lakes

22. Relationship between lake-level improvement and period of pumping.

23. Effects of adding ground water to Eagle Lake on alternate months.

24. Map showing locations of possible damsites........... 68

25. Map showing area that would be inundated by PortageGourdneck Creeks reservoir...........

26. Mathematical model of Kalamazoo River ground-water reservoir and idealized diagram showing practical scheme of development.-..............................

27. Graphs showing water-level fluctuations, pumpage, and precipitation in Kalamazoo River ground-water reservoir, 1946-66 . . . -

28. Mathematical model of Schoolcraft ground-water reservoir and idealized diagram showing practical scheme of development.......................................

29. Mathematical model of Kalamazoo-Portage groundwater reservoir and idealized diagram showing practical scheme of development..............

30-32. Graphs of Kalamazoo-Portage ground-water reservoir showing relationship between water-level fluctuations, pumpage, and precipitation at pumping centers:

30. A for $1963-66$

31. $\mathrm{E}$ for $1960-66$

32. $F$ for 1954-66

33. Graphs showing change in quality and temperature of water from the Upjohn Co.'s well 5

34. Graphs showing dissolved solids and gage height as affected by industrial waste disposal in Portage Creek near Kalamazoo

35. Chemical quality of water in about 280 domestic wells - -

36. Comparison of ground-water withdrawal in 1966 with

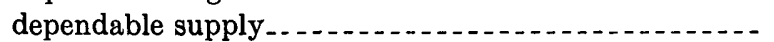

\section{TABLES}

TABLE 1. Summary of hydraulic properties of aquifers and

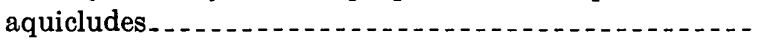

2. Hydrologic budget for Kalamazoo River basin in Kalamazoo County, January 1963 to December 1966..

3. Hydrologic budget for St. Joseph River basin in Kalamazoo County, October 1964 to December 1966........

Page

4. Kalamazoo River discharge and estimated rate of groundwater recharge in Kalamazoo River basin, 1948-62...

5. Portage River discharge and estimated rate of groundwater recharge in St. Joseph River Basin, 1947-51....- 
6. Kalamazoo River discharge and extended estimates of ground-water recharge in Kalamazoo River basin, 1933-47.

Page

7. Source and significance of chemical constituents and physical properties commonly occurring in natural surface and ground water.

8. Selected chemical analyses of ground water, 1960-67 $\ldots$.

9. Streamflow characteristics at gaging and partial-record stations.

10. Estimates of 7- and 30-day low flows for 2- and 10-year recurrence intervals for miscellaneous sites............

11. Chemical quality of water in streams.......

12. Summary of available lake levels . .

13. Inventory of possible damsites.

14. Chemical analyses of lake water

15. Average ground-water pumpage in million gallons per day from ground-water reservoirs and pumping centers, 1960-66

16. Summary of avilability and developmental factors of

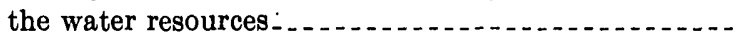





\title{
AVAILABILITY OF WATER IN KALAMAZOO COUNTY, SOUTHWESTERN MICHIGAN
}

\author{
By Willtam B. Allen, John B. Miller, and Warren W. Woon
}

\section{ABSTRACT}

Kalamazoo County comprises an area of $\mathbf{5 7 2}$ square miles in the southwestern part of Michigan. It includes parts of the Klalamazoo, St. Joseph, and Paw Paw River basins, which drain into Lake Michigan. The northern two-thirds of the county is drained by the Kalamazoo River and its tributaries. A small area in the western plart of the county is drained by the Paw Paw River, and the rest, by tributaries of the St. Joseph River. Glacial deposits, containing sand and gravel, form an upper aquifer and a lower aquifer underlying large parts of the county. Areas of high transmissibility and thick saturated deposits are sufficiently localized to be considered as separate ground-water reservoirs having limited areal extent and definite hydrologic boundaries.

Ground-water runoff from the basins constitutes a large part of the streamflow. Hydrograph separation shows that ground-water runoff composed 65 and 73 percent of the discharge of Kalamazoo River at Comstock and 75 and 79 percent of the discharge of Portage River near Vicksburg in 1965 and 1966, respectively. Based on the hydrologic budgets for the same years, ground-water recharge was 9.1 and 9.0 inches in the Kalamazoo River basin and $\mathbf{1 2 . 2}$ and $\mathbf{1 1 . 6}$ inches in the St. Joseph River basin.

Ground-water recharge in the Kalamazoo River basin extrapolated for the 34-year period 1933-66 ranged from 4 to 13 inches and averaged 9 inches. In the St. Joseph River basin average recharge was about 9 inches for the same period.

There is a wide range in runoff in the county. Augusta Creek, Portage Creek near Kalamazoo, and Gourdneck Creek have the highest annual runoff and maintain high yields even during periods of deficient precipitation. Spring Brook also reflects large ground-water contributions to streamflow. Storage in these basins could provide additional water during low flows for municipal and industrial needs.

The primary use of lakes in the county is for recreational and esthetic purposes. Maintaining lake levels is therefore of the utmost importance. Levels at Crooked and Eagle Lakes have been maintained by pumping from lower aquifers. Diversion of water from Gourdneck Creek to West and Austin Lakes has helped in maintaining levels. Several relatively undeveloped lakes could be utilized as reservoirs whose storage could be used to augment streamflow or for water supply. 
Water in streams is generally of good chemical quality; however, several streams, including the Kalamazoo River downstream from Kalamazoo, have been degraded by municipal and industrial waste disposal. Water in the lakes is generally of good chemical quality with the exception of Barton Lake, which has been degraded by waste disposal.

There is sufficient surface water available in Kalamazoo County to meet requirements for development of large quantities of water. The total available supply (average discharge of a stream) is about $680 \mathrm{mgd}$ "(million gallons per day). The dependable supply (7-day $Q_{2}$, or average 7-day low flow having a recurrence interval of 2 years) is about $303 \mathrm{mgd}$. By developing artificial recharge facilities, surface runoff during winter and spring could be utilized to recharge ground-water reservoirs.

Surface-water withdrawal in 1966 was about $58 \mathrm{mgd}$, of which $33 \mathrm{mgd}$ was withdrawn from the Kalamazoo River. The quantity of water now being withdrawn from the ground and surface sources is small compared to the total that may be obtained in the area through full utilization of these resources.

Mathematical models were used to simulate hydrologic conditions in the ground-water reservoirs and to evaluate maximum drawdowns for periods of little or no recharge. The practical limits of development as determined for the ground-water reservoirs are estimated to be at the following average withdrawal rates: Kalamazoo, $39 \mathrm{mgd}$; Schoolcraft, $17 \mathrm{mgd}$; Kalamazoo-Portage, $24 \mathrm{mgd}$; and several small reservoirs, $67 \mathrm{mgd}$. These total $147 \mathrm{mgd}$. Further development. would require additional artificial recharge facilities.

Average ground-water withdrawal in 1966 was about $54 \mathrm{mgd}$. The Kalamazoo River ground-water reservoir furnished about $28 \mathrm{mgd}$, the Kalamazoo-Portage ground-water reservoir, about $21 \mathrm{mgd}$, and the other reservoirs, about $5 \mathrm{mgd}$. Thus, further development without artificial recharge is estimated to be about $11 \mathrm{mgd}$ in the Kalamazoo River reservoir, $17 \mathrm{mgd}$ in the Schoolcraft reservoir, $62 \mathrm{mgd}$ in the several small reservoirs, and only $3 \mathrm{mgd}$ in the KalamazooPortage reservoir.

The ground water is generally of good chemical quality and is suitable for most uses; however, it is usually very hard and may contain objectionable amounts of iron. Some deterioration of water quality. has been observed in several areas because of seepage from stockpiles of industrial minerals.

The presence of many inland lakes, streams having high ground-water runoff, and, in places, relatively undeveloped ground-water reservoirs provides ffexibility in water management.

\section{INTRODUCTION}

Ample supplies of water are vital to the growth and industrial development of Kalamazoo County. Few counties in southern Michigan are blessed with such abundance of well-distributed water resources. The Kalamazoo River with its ample supply is available for dilution of wastes and other industrial uses. Ground water, because of its low content of dissolved solids and its uniform temperature, has special value to municipalities and industry.

In 1963 and 1964 Kalamazoo County experienced a drought. Industry and public supplies were not seriously affected but recreational interests suffered. The levels of Austin, Long, and West Lakes 
declined appreciably. People living on the lakes became concerned. They wanted to know if large ground-water withdrawals by industry were lowering lake levels. Homeowners on Eagle and Crooked Lakes joined together to finance the pumping of ground water to raise lake levels. They were concerned about the effects and effectiveness of programs to maintain lake levels.

In 1964, Kalamazoo County, through the Michigan Department of Conservation, entered into cooperation with the U.S. Geological - Survey to conduct a 4-year investigation of the water resources of the county. That investigation is the basis for this report. At about the same time, the county retained consultants to advise them on planning for future development of the county's resources, including water. It was realized by the consultants that realistic planning would require a knowledge of the total water-supply potential and an appraisal of the impact that future water development would have on existing supplies. The cooperative study was designed to fulfill these needs.

\section{PURPOSE AND SCOPE}

The purpose of this report is to provide a technical appraisal of the water facts that will aid in planning the orderly development of the water resources of the county and will serve as a guide for their management.

The report (1) defines the relationship between surface and subsurface water units, (2) maps variations in water-yielding characteristics of principal ground-water units, (3) describes the availability of water in streams, (4) identifies and shows possible surface-storage sites and estimates how storage can be used to augment streamflow, (5) determines the average annual recharge by means of water budgets utilizing precipitation, temperature, streamflow, and waterlevel data, (6) defines the physical and chemical quality of ground and surface water and what these mean to water users, (7) defines present and potential development of the principal subsurface reservoirs and existing pumping centers with the use of mathematical models, (8) defines areas favorable for development of additional centers of pumping and their practical limits of development, and (9) - evaluates problems that may arise with extensive subsurface reservoir development, such as anticipated changes in streamflow, use of artificial recharge ponds, and water quality.

\section{ACKNOWLEDGMENTS AND PERSONNEL}

The authors thank all persons who contributed information and assistance during the collection, processing, and evaluation of data used in this report. The contributions by the following persons, how- 
ever, warrant special mention: Albert Sabo and Thomas E. Fricke, Utilities Department, City of Kalamazoo; Walker H. Sisson and Frederick M. Rueber, Upjohn Co.; William J. Zinkus, KVP Division, Brown Co.; and Edward N. Ross, Simpson-Lee Paper Co., furnished records of pumpage, production wells, and test holes. Ernest L. Bockstanz, formerly with Kalamazoo County Road Commission, furnished test-hole data and permission to drill test wells and install gaging stations on county land. Abdel Wahid Ibrahim Abdel Wahid, a graduate student at Michigan State University, was helpful in providing data from gravity meter and resistivity surveys.

Many U.S. Geological Survey personnel assisted in the investigation. John R. Rapp and Earl L. Skinner served as project personnel during the early stages of the investigation. Special credit is due to Ted Thompson, who assisted in the collection of the field data, the analyses and computations of data, and the preparation of illustrations.

Herbert O. Larkin, Engineer-Manager, Kalamazoo County Road Commission, and Bruce A. Watts, Director, Kalamazoo County Planning Department, acted as coordinators for the investigation.

Many of the data used in this study were collected over a period of years by the U.S. Geological Survey in cooperation with State agencies, particularly the Michigan Geological Survey, Water Resources Commission, State Highway Department, and State Health Department.

The investigation was made by the U.S. Geological Survey under the direction of A. D. Ash, District Chief, and the general supervision of G. E. Hendrickson, Associate District Chief.

\section{LOCATION}

Kalamazoo County, in southwestern Michigan, includes parts of the Kalamazoo, St. Joseph, and Paw Paw River basins, which drain into Lake Michigan (fig. 1). It is about 24 miles square and has an area of about $572 \mathrm{sq}$ mi (square miles).

\section{TOPOGRAPHY}

The northern half of Kalamazoo County is characterized by irregular hilly areas. The rolling highlands area in the northwest quarter is a part of the Kalamazoo moraine. Altitudes along the moraine reach more than 1,000 feet above sea level. The highland in the northeast is a dissected outwash plain. The remnants of the plain are at altitudes of about 960 feet. The streams dissecting the plain have entrenched as much as 160 feet below these remnants. The central and southwest parts of the county are largely a lower-level outwash plain cut and filled by stream channels; the southeast quarter is a 


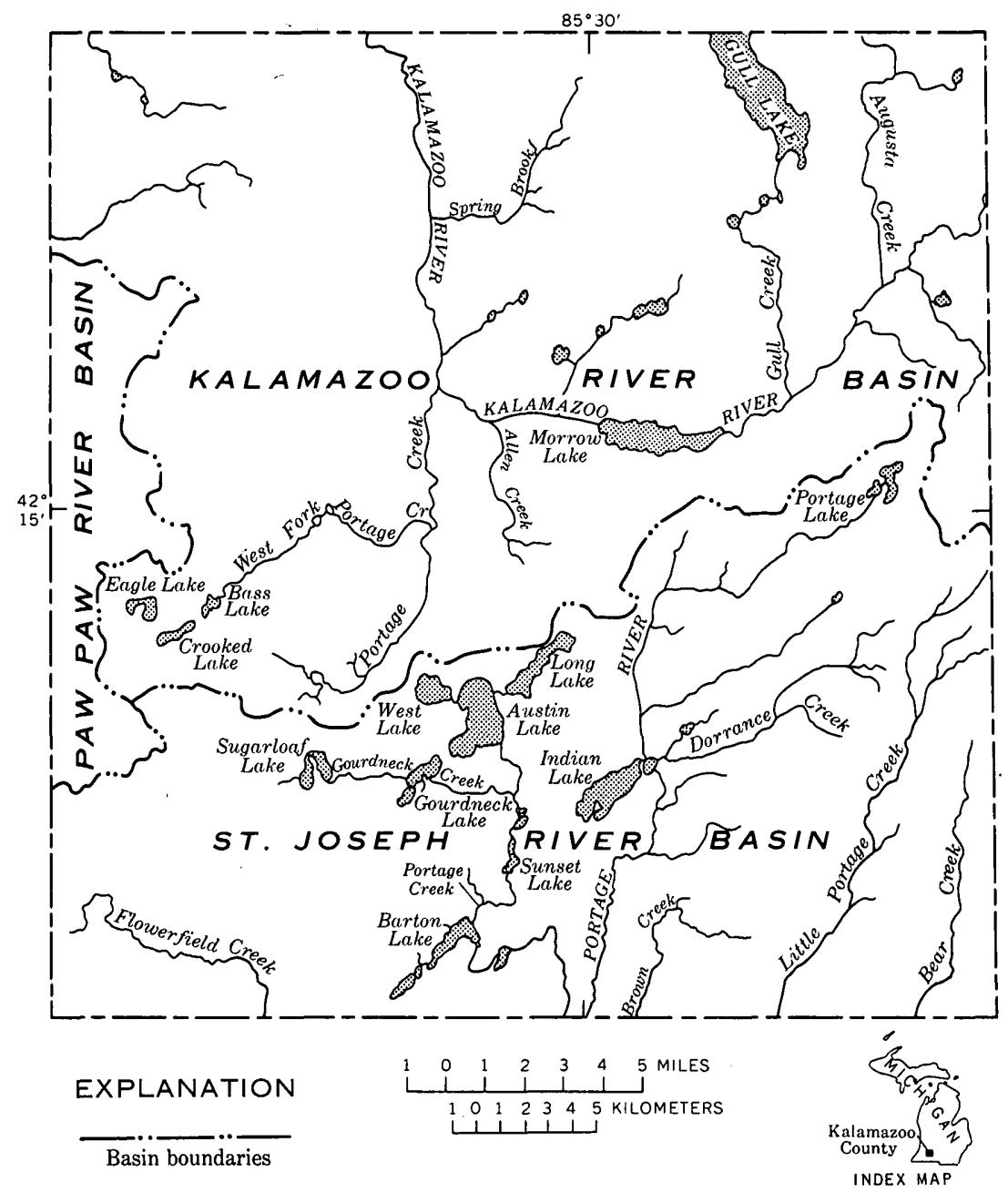

FTGURE 1.-Location of Kalamazoo County and its surface-water system.

rolling till plain. The total relief of the land surface in the county is about 300 feet. The altitude ranges from about 740 feet where the Kalamazoo River leaves the county to about 1,040 feet on the hilltops.

\section{CLIMATE}

Lake Michigan, about 25 miles west of Kalamazoo County, has a general moderating effect upon the climate of the county. Climatic records collected at Kalamazoo State Hospital since 1867, which are considered to be representative of conditions in the county, indicate that mean monthly temperatures range from $27^{\circ} \mathrm{F}$ in February to $74^{\circ} \mathrm{F}$ in July. The records also show that the average annual tem- 
perature is $49.6^{\circ} \mathrm{F}$ and that the growing season is about 5 months, the average dates of the last spring and first fall temperatures of $32^{\circ} \mathrm{F}$ or colder being May 9 and October 9, respectively. Annual precipitation at Kalamazoo State Hospital averages about 35 inches and ranged from about 21 to 42 inches during the 30 -year period 1931-60. During that period the average annual precipitation had been equaled or exceeded about 60 percent of the time. Snowfall averages about 55 inches per winter season.

This study was initiated in 1964, when lake and ground-water levels had receded to record lows owing to several years of deficient precipitation. Deficiencies in precipitation began in July 1960 and continued until July 1965, when the cumulative departure was 19.7 inches below normal for the period 1931-60 (fig. 2). Since 1965, precipitation has been increasing, and by 1968 the deficiency in precipitation was only about 6 inches below normal.

Precipitation is not evenly distributed in Kalamazoo County largely because of the influence of Lake Michigan and differences in land-surface altitudes. It is generally greater in the western morainal (upland) part of the county than in the central and southern lowlands (fig. 3). The years 1963 and 1964 are a part of the drought period; 1965 and 1966. were above average in precipitation.

\section{HYDROLOGIC SYSTEM}

Water occurs in Kalamazoo County in streams, lakes, and marshes and in the glacial deposits, alluvium, and bedrock that underlie the county. The streams, lakes, and marshes form a surface-water system; the unconsolidated deposits and consolidated rocks together form a ground-water system. The surface-water and ground-water parts of the water system are hydraulically interconnected, and water moves from one to the other in response to both natural and man-made hydraulic gradients. A discussion of the two systems and their relationship to each other follows.

\section{SURFACE-WATER SYSTEM}

The largest stream in Kalamazoo County is the Kalamazoo River. It flows westward to the center of the city of Kalamazoo and then northward out of the county. Its principal tributaries from the north and east are Augusta Creek, Gull Creek, and Spring Brook; from the south, they are Allen and Portage Creeks. Principal streams in the south half of the county are Little Portage Creek, Portage River, Flowerfield Creek, and Gourdneck Creek-Portage Creek-all part of the St. Joseph River basin. Headwaters of the Paw Paw River drain the extreme western part of the county (fig. 1). 


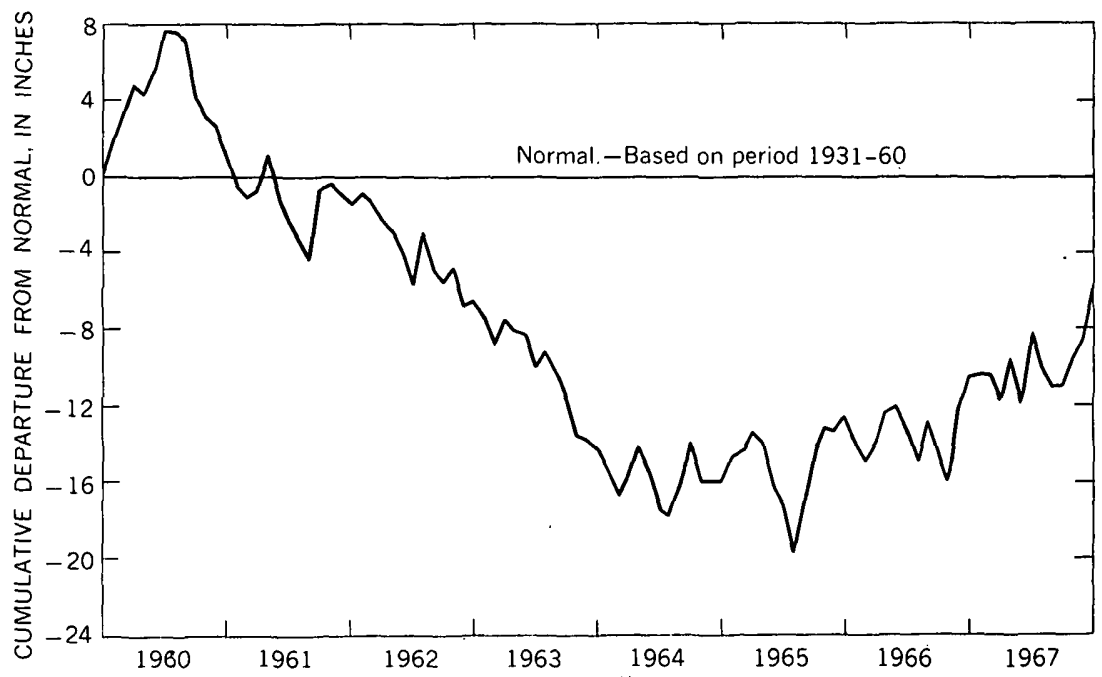

FiguRe 2.-Cumulative monthly precipitation departure from long-term monthly norms:

There are 356 lakes and ponds in the county (Humphrys and Green, 1962) ranging in size from less than an acre to 2,050 acres. The largest lake is Gull Lake, located in the northeastern part of the county. The other large lakes are Indian, Long, Austin, West, Gourdneck, and Barton Lakes (fig. 1). Their use is for recreational and esthetic values. Morrow Lake is an impoundment of the Kalamazoo River 1 mile above Comstock center. The total storage capacity of this impoundment is only about 4,000 acre- $\mathrm{ft}$ :

Marshes in Kalamazoo County are characterized by decayed organic matter (peat and muck) mixed with and generally underlain by silt, clay, and fine sand. They are along streams and around the many lakes, particularly in the lowlands in the south-central part of the county.

\section{GROUND-WATER SYSTEM}

The geologic materials making up the subsurface system underlying Kalamazoo County are of two types: (1) unconsolidated deposits of gravel, sand, silt, and clay, and (2) bedrock (consolidated rocks) composed of shale, limestone, sandstone, and other types of 'sedimentary rocks. The physical characteristics and areal extent of these materials in a large part of the county have been described in some detail by Deutsch, Vanlier, and Giroux (1960). Of these materials, only the unconsolidated sand and gravel deposits have sufficient permeability to yield appreciable quantities of water to wells. They form the principal aquifers and constitute the subsurface reservoirs referred to in the quantitative appraisal. 

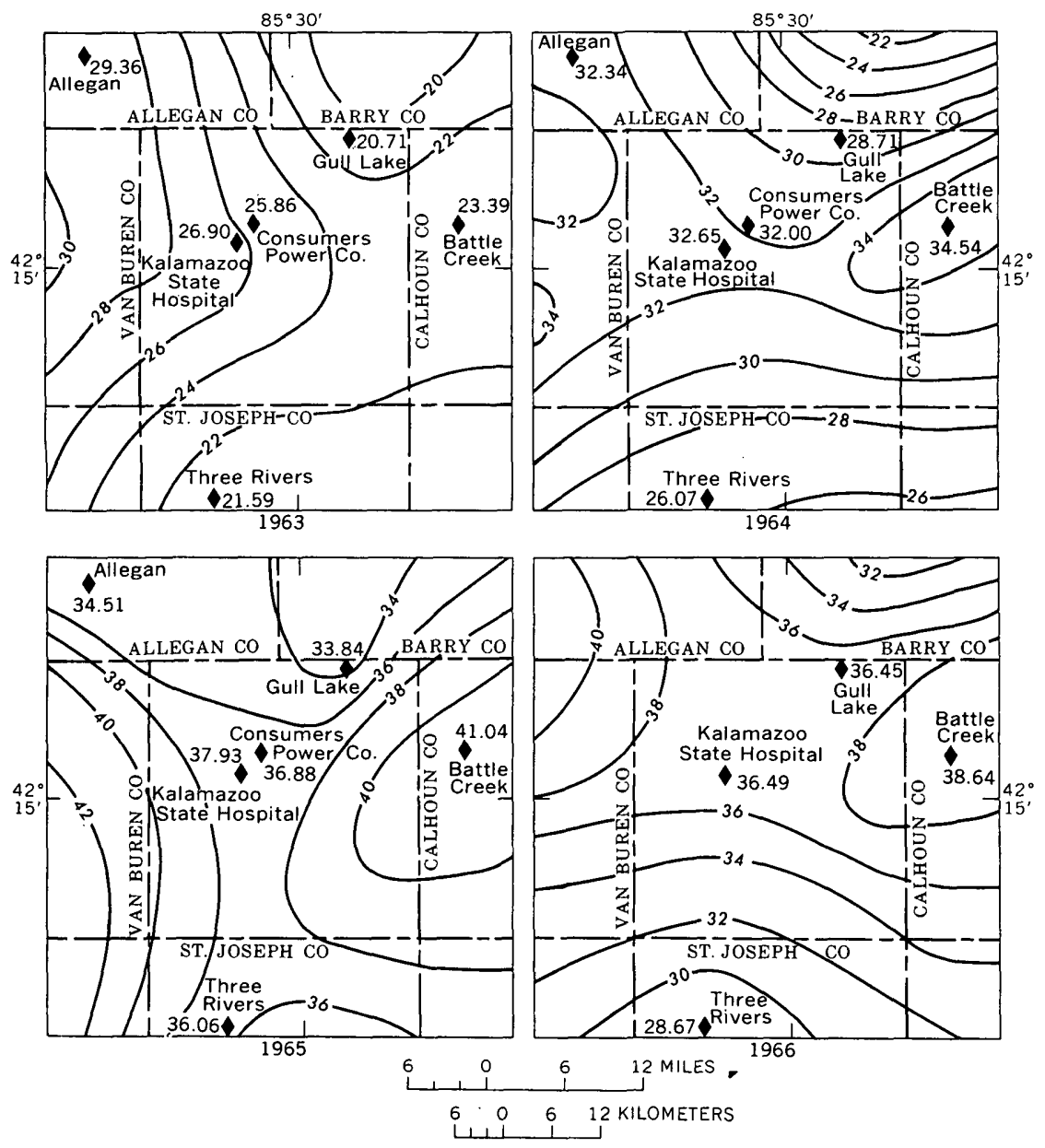

EXPLANĄTION

33.84

Gull Lake

Precipitation station

$\Lambda^{\top} u m b e r$ indicates precipitation, in inches.

IGURE 3.-Distribution of annual precipitation in Kalamazoo County and vicinity, 1963-66.

\section{PHYSICAL CHARAGTERISTICS OF AQUIFERS}

An aquifer is defined as a water-yielding deposit or formation. An aquiclude is a deposit or formation which, although porous, will not yield an appreciable supply of water to a well or spring. The function of an aquifer is to store and transmit water; that of an aquiclude is to impede the vertical and horizontal movement of water. In 
Kalamazoo County, the deposits predominantly of sand and gravel, with minor amounts of fine sand, silt, and clay, that yield large quantities of water comprise the aquifers; the deposits or combinations of them which yield only small quantities of water are the aquicludes. Lenses of poorly sorted fine materials of low permeability are present in the aquifers and lenses of well-sorted, coarse materials of high permeability are present in the aquicludes. Thus, it is possible to obtain small amounts of water, enough for domestic needs, from any of the saturated unconsolidated deposits in Kalamazoo County.

Because of the variation in sorting and stratification, the unconsolidated deposits in Kalamazoo County vary in permeability both vertically and horizontally. They are, however, separated into four general units-an upper aquifer, an intervening aquiclude or semiconfining layer, a lower aquifer, and a basal aquiclude or confining layer. The location and saturated thickness of the upper and lower aquifers and the relationship of the aquicludes to the upper and lower aquifers are shown on plate 1. Twenty-three lithologic sections in northwest-southeast and northeast-southwest directions were prepared to show the physical characteristics of the unconsolidated materials throughout the county. The sections were then generalized and used to delineate the upper and lower aquifers and the aquicludes. The saturated thicknesses of the aquifers, which were determined from the sections, are shown on plate 1. For areas having two lower aquifers, the map shows the combined thickness of these aquifers. Saturated thickness is important because it is needed to determine the amount of drawdown that is available for development and the volume of water in storage. Sections $A-A^{\prime}, B-B^{\prime}$, and $C-C^{\prime}$ pass through the thickest parts of the lower aquifer. The saturated thickness ranges from zero to more than 140 feet in the upper aquifer and from zero to 160 feet in the lower aquifer.

\section{HYDRAULIC PROPERTIES OF AQUIFERS}

The significant hydraulic properties of the upper and lower aquifers are summarized in table 1. Plate 2 shows the transmissibility of the aquifers. The water-transmitting capacity of an aquifer is referred to as its transmissibility. The coefficient of transmissibility is defined as the number of gallons of water transmitted per day through a vertical strip 1 foot wide and total aquifer thickness in height under a hydraulic gradient of 1 foot per foot. It is reported in gallons per day per foot. Specific capacity is the yield of a well, in gallons per minute, divided by the drawdown in the well, in feet. The data used to define the transmissibility of the aquifers were 
TABLE 1.-Summary of hydraulic properties of aquifers and aquicludes

[Wells are numbered from left to right by township, range, and section; the fourth number is well number in section. TD, time-drawdown; DD, distance-drawdown; SC, specific capacity]

\begin{tabular}{|c|c|c|c|c|c|c|}
\hline Well No. and location & $\begin{array}{c}\text { Coefficient } \\
\text { of trans- } \\
\text { missibility } \\
\text { (gpd per } \\
\text { ft) }\end{array}$ & $\begin{array}{c}\text { Coefficient } \\
\text { of } \\
\text { storage }\end{array}$ & $\begin{array}{l}\text { Coefficient of } \\
\text { permeability } \\
\text { (gpd per } \\
\text { sq ft) }\end{array}$ & $\begin{array}{c}\text { Coefficient } \\
\text { of vertical } \\
\text { permeability } \\
\text { of aquicludes } \\
\text { (gpd per } \\
\text { sq ft) }\end{array}$ & $\begin{array}{c}\text { Method of } \\
\text { analysis }\end{array}$ & Aquifer \\
\hline $\begin{array}{r}1-9-29-5 \\
10-14-3 \\
11-34-7 \\
34-40 \\
2-11-6-1 \\
11-14-5 \\
20-12 \\
22-4 \\
29-2 \\
5 \\
31-2 \\
3-11-3-3 \\
11-4-3 \\
4-10 \\
40 \\
42 \\
9-2 \\
14-101 \\
12-11-4 \\
4-11-13-34 \\
11-21-4 \\
12-25-3 .\end{array}$ & $\begin{array}{r}150,000 \\
71,000 \\
190,000 \\
130,000 \\
35,000 \\
80,000 \\
60,000 \\
79,000 \\
44,000 \\
46,000 \\
110,000 \\
40,000 \\
105,000 \\
52,000 \\
110,000 \\
100,000 \\
128,000 \\
84,000 \\
150,000 \\
54,000 \\
100,000 \\
100,000\end{array}$ & $\begin{array}{l}0.02 \\
.00006 \\
.02 \\
.0051 \\
.0024 \\
.00057 \\
.19 \\
.00047 \\
.0037 \\
.0018 \\
.0024 \\
.0037 \\
.0016 \\
.03 \\
.04 \\
.0002\end{array}$ & $\begin{array}{l}1,600 \\
1,900 \\
2,300 \\
2,600 \\
1,700 \\
2,600 \\
2,400 \\
800 \\
1,200 \\
900 \\
3,100 \\
700 \\
2,400 \\
3,600 \\
3,200 \\
2,800 \\
800 \\
2,800 \\
3,000 \\
800 \\
1,300 \\
1,100\end{array}$ & 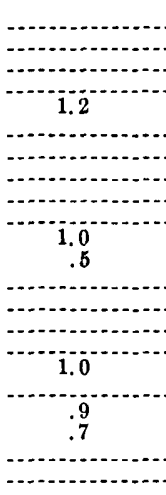 & $\begin{array}{l}\text { TD } \\
\text { TD } \\
\text { TD } \\
\text { TD } \\
\text { TD } \\
\text { TD } \\
\text { TD } \\
\text { TD } \\
\text { TD } \\
\text { TD, DD } \\
\text { TD } \\
\text { TD } \\
\text { TD } \\
\text { TD } \\
\text { TD } \\
\text { TD, DD } \\
\text { TD } \\
\text { SC } \\
\text { SC }\end{array}$ & $\begin{array}{l}\text { Upper. } \\
\text { Do. } \\
\text { Do. } \\
\text { Do. } \\
\text { Lower. } \\
\text { Upper. } \\
\text { Lower. } \\
\text { Do. } \\
\text { Do. } \\
\text { Do. } \\
\text { Do. } \\
\text { Do. } \\
\text { Do. } \\
\text { Do. } \\
\text { Upper. } \\
\text { Lower. } \\
\text { Do. } \\
\text { Do. } \\
\text { Do. } \\
\text { Do. } \\
\text { Do. } \\
\text { Do. }\end{array}$ \\
\hline
\end{tabular}

obtained, in part, from the analyses of pumping tests and specific capacity tests of wells in table 1 . The theory on which the determinations of transmissibility are based and the methods of analysis are described in detail in Bentall (1963a, b, c), Bolton (1963), Ferris, Knowles, Brown, and Stallman (1962), Walton (1962), Weeks (1964), and Csallany (1966).

Additional estimates of transmissibility were determined from the materials penetrated by wells. Computations were based on the relation between transmissibility $(T)$, permeability $(P)$, and saturated thickness $(m): T=P m$. Multiple regression analysis, reported by Jenkins (1963), was used to determine some of the relationships between the logs of materials and the hydrologic properties shown in table 1. Using these relationships, representative values of permeability could be assigned to the materials described in logs. Values obtained from the analysis are listed in the following table and are

Material

Clay and silt

Very fine sand

Fine sand

Fine to medium sand

Medium sand

Medium to coarse sand

Coarse sand

Very coarse sand

Sand and gravel

Gravel

Till
Permeability

(gpd per $s q f t)$

0-100

100-200

200-400

400-500

*500-600

600-700

*700-800

800-1,000

$* 1,000-2,500$

$* 2,500-5,000$

20-500

*Based on graphical multiple regression analyses of 22 pumping tests. 
judged to be applicable to the unconsolidated deposits underlying Kalamazoo County.

Permeability is defined as the capacity of a material to transmit a fluid. The coefficient of permeability is the rate of flow of water in gallons per day through a cross-sectional area of 1 square foot under a hydraulic gradient of 1 foot per foot at a temperature of $60^{\circ} \mathrm{F}$ $\left(15.6^{\circ} \mathrm{C}\right)$; it is reported as gallons per day per square foot. The field coefficient of permeability is the same, except that it is measured under prevailing conditions, particularly as to temperature of the water. Water.is stored in the sand, gravel, and other porous materials. The volume of water in storage is represented by the storage coeffcient, which is defined as the volume of water, expressed as a decimal fraction of a cubic foot, released from storage in a column of the aquifer having a cross-sectional area of 1 square foot and a height equal to the full thickness of the aquifer when the head is lowered 1 foot.

The permeabilities were arbitrarily adjusted downward when applied to logs describing sand and gravel interbedded with relatively thick lenses of till or fine sand, silt, and clay. The map shows that the transmissibility in the upper aquifer ranges from less than 20,000 to more than $140,000 \mathrm{gpd}$ per $\mathrm{ft}$ and in the lower aquifer ranges from less than 20,000 to more than $180,000 \mathrm{gpd}$ per ft. Relatively large areas of high transmissibility, generally greater than 40,000 gpd per $\mathrm{ft}$, are delineated by rectangles on the map. Outward from each rectangular area the transmissibility decreases markedly towards the boundary of the aquifer. The areas selected on the basis of high transmissibility and thick saturated material are considered herein to be the ground-water reservoirs. These areas, named as reservoirs for their geographic location, are shown on plate 2 . The present and potential yields of each of these reservoirs are described under the section "Ground-water reservoirs."

\section{GROUND-WATER-SURFACE-WATER RELATIONSHIPS}

Water moves freely between the surface-water and ground-water systems. The normal dry-weather flow of streams is maintained entirely by ground-water discharge, and in some areas water is lost from streams to recharge the aquifers. Water also moves from aquifers to lakes and from lakes to aquifers. These relationships must be understood before quantitative appraisal of the ground-water reservoirs can be made. They also provide insight on how the system operates and how it can be controlled or managed. 


\section{RECHARGE FROM PRECIPITATION AND THE HYDROLOGIC BUDGET}

Recharge to aquifers is derived from infiltration of precipitation and, locally, from infiltration of surface water. The term recharge as used in this section of the report includes only recharge from precipitation. Most recharge occurs during seasons of low evapotranspiration; some recharge may occur during any month. It is generally greatest from November through May in Kalamazoo County. The amount of recharge can be estimated by a ground-water budget analysis, if the ground-water runoff can be defined. The accounting, which includes precipitation, total runoff, and water loss, is commonly referred to as a hydrologic budget. Water loss has been computed as the difference between precipitation and total runoff. It does not include that water which goes into storage. The ground-water part of the budget can be expressed by the following relationship: groundwater recharge equals ground-water runoff plus ground-water evapotranspiration plus or minus change in ground-water storage plus or minus subsurface underflow (Rasmussen and Andreasen, 1959; Schicht and Walton, 1961; and Olmsted and Hely, 1962). Underflow is that part of the ground-water discharge that leaves the basin underground.

In Kalamazoo County, ground-water budgets for the Kalamazoo and St. Joseph River basins were prepared to obtain estimates of ground-water recharge. These estimates were computed on a monthly basis and are shown in tables 2 and 3.

The ground-water runoff part of the budget was determined using streamflow records for Kalamazoo River at Comstock and Portage River near Vicksburg. To do this, stream discharge was separated into its components of surface runoff and ground-water runoff. Separation of the hydrographs for the two rivers is shown on plate 3 . The component of ground-water runoff was determined by using base-flow recession curves (curves which show the rate at which streamflow declines during periods of little or no precipitation). Also utilized in the separation were records of ground-water levels and climatic data.

The hydrograph separation technique indicates that 65 percent of the total flow in the Kalamazoo River consisted of ground-water discharge in 1965 ; in 1966, 73 percent was ground-water discharge. In the St. Joseph River basin for the same years, 75 and 79 percent were ground-water discharge. Ground-water discharge figures include some runoff that is held in temporary storage in marshes and ponds and as channel storage. This is particularly true during the spring; however, during the summer and fall nearly all the runoff is groundwater discharge. Also, because the Kalamazoo River is a large stream 
in a humid region, the separation of surface runoff and ground-water runoff may be somewhat in error. However, it seems fairly safe to assume that, on an annual basis, the estimated ground-water runoff is probably fairly accurate.

The ground-water evapotranspiration part of the budget was estimated from curves prepared to show the relationship between mean ground-water stage and ground-water runoff in a manner similar to that used in the Brandywine Creek basin of Pennsylvania (Olmsted and Hely, 1962). Months with little or no evapotranspiration tend to plot to the right and months with evapotranspiration, to the left. A straight line drawn through the months to the right represents the line of no evapotranspiration. The deviation of the monthly plots from the line give the amount of evapotranspiration as shown in tables 2 and 3.

Monthly change in storage was determined as the difference in ground-water level between the beginning of the month and the end of the month multiplied by a storage coefficient. The coefficient 0.12 used in these computations was selected as representative of a basinwide average and is not indicative of the coefficients that apply to individual aquifers.

There probably are errors either in ground-water evaporation or in the change in storage that reflect inaccuracies inherent in the methods of computation.

Subsurface underflow bypassing each gaging station was computed using the appropriate data from figures 5 and 10 and a form of Darcy's equation,

$$
Q=P I A,
$$

where $Q=$ the quantity of subsurface flow,

$P=$ the coefficient of permeability,

$I=$ the hydraulic gradient, and

$A=$ the cross-sectional area.

The underflow at Kalamazoo River and at Portage River gaging stations was negligible-only 0.01 inch per year, each.

The amount of recharge in the Kalamazoo River basin during the years 1963 and 1964 was less than that in 1965. That for 1965 and 1966 was about the same as could be expected in normal years. In the St. Joseph River basin during the years 1965 and 1966, recharge was greater than would be expected in normal years. In order to determine normal recharge, annual ground-water budgets were computed for the period 1948-62 in the Kalamazoo basin and for 1947-51 in the St. Joseph basin (tables 4 and 5). Budgets were computed using extended water-level data to represent a longer period, and climatic and streamflow records as described on page 12. In addition, esti- 


\section{4}

WATER AVAILABILITY, KALAMAZOO COUNTY, MICHIGAN

\section{TABLE 2.-Hydrologic budget for Kalamazoo River basin in Kalamazoo County January 1963 to December 1966}

[A]l units expressed in inches, except where noted. Precipitation data are averages from records at U.S. Weather Bureau station 0552 at Battle Creek, Mich., station 3504 at Gull Lake, Mich., and station 4244 at Kalamazoo State Hospital, Kalamazoo, Mich. Water losses are the difference between precipitation and total runoff and include evapotranspiration and soll-moisture losses. Total-runoff data are from records at U.S. Geological Survey gaging station 4-1060 on Kalamazoo River at Comstock, Mich. Surface runoff is the difference between total runoff and ground-water runoff. Change in storage is based on change in average water level in wells 1-7-10-1 and 2-10-13-5. Surface drainage area is $1,010 \mathrm{sq} \mathrm{mi}$, approximately. Ground-water recharge per square mile: mgd, million gallons per day. Negative values of recharge excluding underflow are attributed to errors in estimating budget elements.]

\begin{tabular}{|c|c|c|c|c|c|c|c|c|c|}
\hline & \multirow{2}{*}{$\begin{array}{c}\text { Preclpi- } \\
\text { tation }\end{array}$} & \multirow{2}{*}{$\begin{array}{l}\text { Water } \\
\text { losses }\end{array}$} & \multirow{2}{*}{$\begin{array}{l}\text { Total } \\
\text { runoff }\end{array}$} & \multirow{2}{*}{$\begin{array}{l}\text { Surface } \\
\text { runoff }\end{array}$} & \multicolumn{5}{|c|}{ Ground-water budget } \\
\hline & & & & & Runoff & $\begin{array}{l}\text { Evapotrans- } \\
\text { fir piration }\end{array}$ & $\begin{array}{c}\text { Change in } \\
\text { storage }\end{array}$ & $\begin{array}{l}\text { Recharge } \\
\text { excluding } \\
\text { underflow }\end{array}$ & $\begin{array}{l}\text { Recharge } \\
\text { per sq mi } \\
\text { (mgd) }\end{array}$ \\
\hline 1968 & & & . & & & & & & \\
\hline 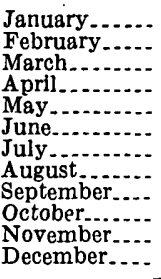 & $\begin{array}{l}1.15 \\
.60 \\
3.00 \\
2.46 \\
3.81 \\
1.88 \\
3.81 \\
2.01 \\
1.14 \\
1.10 \\
1.66 \\
1.05\end{array}$ & $\begin{array}{r}0.67 \\
.19 \\
1.73 \\
1.70 \\
3.03 \\
1.45 \\
3.42 \\
1.64 \\
.83 \\
.79 \\
1.35 \\
.65\end{array}$ & $\begin{array}{l}0.48 \\
.41 \\
1.27 \\
.76 \\
.78 \\
.43 \\
.39 \\
.37 \\
.31 \\
.31 \\
.31 \\
.40\end{array}$ & $\begin{array}{l}0.02 \\
.01 \\
.74 \\
.18 \\
.24 \\
.02 \\
.03 \\
.03 \\
.01 \\
.01 \\
.01 \\
.03\end{array}$ & $\begin{array}{l}0.46 \\
.40 \\
.53 \\
.58 \\
.54 \\
.41 \\
.36 \\
.34 \\
.30 \\
.30 \\
.30 \\
.37\end{array}$ & $\begin{array}{c}\text { Negligible... } \\
0.030 \\
0.07 \\
0.15 \\
0.19 \\
0.16 \\
0.15 \\
0.11 \\
0.08 \\
\text { Negligible... }\end{array}$ & $\begin{array}{r}-0.22 \\
-.22 \\
+1.65 \\
-.07 \\
-.29 \\
-.14 \\
-.22 \\
-.43 \\
-.43 \\
-.43 \\
-.14 \\
-.14\end{array}$ & $\begin{array}{r}0.24 \\
.18 \\
2.18 \\
.54 \\
.32 \\
.42 \\
.33 \\
.07 \\
.02 \\
-.02 \\
.24 \\
.23\end{array}$ & $\begin{array}{l}0.13 \\
.11 \\
2.71 \\
.31 \\
.18 \\
.24 \\
.18 \\
.04 \\
.01 \\
.00 \\
.14 \\
.13\end{array}$ \\
\hline Total.... & 23.67 & 17.45 & 6. 22 & 1.33 & $4.89 \quad 0$ & $0.94 \ldots \ldots$ & -1.08 & 4.75 & $\ldots$ \\
\hline
\end{tabular}

1964

\begin{tabular}{|c|c|c|c|c|c|c|c|c|c|}
\hline $\begin{array}{l}\text { January } \\
\text { February } \\
\text { March.... } \\
\text { April... } \\
\text { May } \\
\text { June.... } \\
\text { July } \\
\text { August } \\
\text { September.... } \\
\text { October } \\
\text { November... } \\
\text { December... }\end{array}$ & $\begin{array}{r}.86 \\
.52 \\
2.85 \\
4.22 \\
2.59 \\
2.87 \\
3.13 \\
4.87 \\
4.67 \\
1.14 \\
2.47 \\
1.77\end{array}$ & $\begin{array}{l}.44 \\
.12 \\
2.32 \\
3.54 \\
2.00 \\
2.50 \\
2.81 \\
4.58 \\
4.32 \\
.78 \\
2.12 \\
1.22\end{array}$ & $\begin{array}{l}.42 \\
.40 \\
.53 \\
.68 \\
.59 \\
.37 \\
.32 \\
.29 \\
.35 \\
.36 \\
.35 \\
.55\end{array}$ & $\begin{array}{l}.05 \\
.04 \\
.07 \\
.19 \\
.14 \\
.01 \\
.02 \\
.01 \\
.02 \\
.03 \\
.02 \\
.17\end{array}$ & $\begin{array}{l}.37 \\
.36 \\
.46 \\
.49 \\
.45 \\
.36 \\
.30 \\
.28 \\
.33 \\
.33 \\
.33 \\
.38\end{array}$ & $\begin{array}{c}\text { Negligible... } \\
0.03 \text { do } \\
0.10 \\
0.16 \\
0.16 \\
0.12 \\
0.03 \\
0.02 \\
0.02 \\
\text { Negligible... }\end{array}$ & $\begin{array}{l}-.07 \\
+.07 \\
+.07 \\
+.29 \\
-.07 \\
-.29 \\
-.86 \\
-.43 \\
-.22 \\
-.50 \\
+.50 \\
+.43\end{array}$ & $\begin{array}{r}.30 \\
.43 \\
.53 \\
.81 \\
.48 \\
.23 \\
-.40 \\
-.03 \\
.14 \\
-.15 \\
.85 \\
.81\end{array}$ & $\begin{array}{l}.17 \\
.26 \\
.30 \\
.47 \\
.27 \\
.13 \\
.00 \\
.00 \\
.00 \\
.00 \\
.49 \\
.45\end{array}$ \\
\hline Tote & 31.96 & 26.75 & 5.21 & .77 & 4.44 & $0.64 \ldots$ & -1.08 & 4.00 & \\
\hline
\end{tabular}

\section{5}

\begin{tabular}{|c|c|c|c|c|c|c|c|c|c|}
\hline 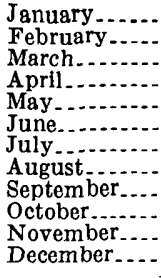 & $\begin{array}{l}3.16 \\
2.06 \\
2.97 \\
2.22 \\
2.02 \\
3.14 \\
1.67 \\
5.00 \\
5.49 \\
2.32 \\
2.58 \\
4.98\end{array}$ & $\begin{array}{l}2.49 \\
1.06 \\
1.61 \\
.82 \\
1.40 \\
2.59 \\
1.29 \\
4.58 \\
5.01 \\
1.79 \\
2.11 \\
4.06\end{array}$ & $\begin{array}{l}.67 \\
1.00 \\
1.36 \\
1.40 \\
.62 \\
.55 \\
.38 \\
.42 \\
.48 \\
.53 \\
.47 \\
.92\end{array}$ & $\begin{array}{l}.20 \\
.56 \\
.82 \\
.79 \\
.05 \\
.09 \\
.01 \\
.07 \\
.05 \\
.05 \\
.01 \\
.35\end{array}$ & $\begin{array}{l}.47 \\
.44 \\
.54 \\
.61 \\
.57 \\
.46 \\
.37 \\
.35 \\
.43 \\
.48 \\
.46 \\
.57\end{array}$ & $\begin{array}{c}\text { Negligible.... } \\
0.04 \\
0.09 \\
0.16 \\
0.22 \\
0.19 \\
0.09 \\
0.06 \\
0.03 \\
\text { Negligible... }\end{array}$ & $\begin{array}{r}+.79 \\
+.36 \\
+.94 \\
+.79 \\
-.36 \\
-.50 \\
-.43 \\
-.29 \\
+.29 \\
-.43 \\
+.14 \\
+1.15\end{array}$ & $\begin{array}{l}1.26 \\
.80 \\
1.48 \\
1.44 \\
.30 \\
.12 \\
.16 \\
.25 \\
.81 \\
.11 \\
.63 \\
1.72\end{array}$ & $\begin{array}{l}.70 \\
.49 \\
.82 \\
.83 \\
.17 \\
.07 \\
.00 \\
.14 \\
.47 \\
.06 \\
.36 \\
.96\end{array}$ \\
\hline Total. & 37.61 & 28.81 & 8.80 & 3. 05 & 5.75 & $0.88 \ldots$ & +2.45 & 9.08 & \\
\hline $\begin{array}{l}\quad 1966 \\
\text { January } \\
\text { February } \\
\text { March } \\
\text { April } \\
\text { May } \\
\text { June } \\
\text { July } \\
\text { August } \\
\text { September } \\
\text { October } \\
\text { November } \\
\text { December } \\
\end{array}$ & $\begin{array}{l}.83 \\
1.54 \\
3.12 \\
4.73 \\
4.20 \\
2.40 \\
1.89 \\
5.48 \\
1.61 \\
1.36 \\
6.06 \\
3.97\end{array}$ & $\begin{array}{r}-.05 \\
.81 \\
1.99 \\
3.68 \\
2.81 \\
1.74 \\
1.40 \\
4.98 \\
1.23 \\
.93 \\
5.38 \\
2.84\end{array}$ & $\begin{array}{r}.88 \\
.73 \\
1.13 \\
1.05 \\
1.39 \\
.66 \\
.49 \\
.50 \\
.38 \\
.43 \\
.68 \\
1.13\end{array}$ & $\begin{array}{l}.24 \\
.13 \\
.44 \\
.36 \\
.61 \\
.03 \\
.01 \\
.10 \\
.01 \\
.02 \\
.16 \\
.45\end{array}$ & $\begin{array}{l}.64 \\
.60 \\
.69 \\
.69 \\
.78 \\
.63 \\
.48 \\
.40 \\
.37 \\
.41 \\
.52 \\
.68\end{array}$ & $\begin{array}{l}\text { Negligible.... } \\
0.02 \\
0.03 \\
0.14 \\
0.26 \\
0.28 \\
0.27 \\
0.23 \\
0.10 \\
\text { Negligible.... }\end{array}$ & $\begin{array}{l}-.07 \\
+.50 \\
+.22 \\
+.94 \\
+.50 \\
-.65 \\
-.58 \\
-.29 \\
-.50 \\
-.07 \\
.00 \\
+.79\end{array}$ & $\begin{array}{l}.57 \\
1.10 \\
.91 \\
1.65 \\
1.31 \\
.12 \\
.16 \\
.39 \\
.14 \\
.57 \\
.62 \\
1.47\end{array}$ & $\begin{array}{l}.32 \\
.68 \\
.51 \\
.95 \\
.73 \\
.07 \\
.09 \\
.22 \\
.08 \\
.32 \\
.36 \\
.82\end{array}$ \\
\hline Total..... & 37. 19 & 27.74 & 9.45 & 2. 56 & 6.89 & $1.33 \ldots \ldots$ & +.79 & 9.01 & \\
\hline
\end{tabular}


TABLF 3.-Hydrologic budget for St. Joseph River basin in Kalamazoo County, October 1964 to December 1966

[All units expressed in inches, except where noted. Precipitation data are averages from records at U.S. Weather Bureau station 0552 at Battle Creek, Mich., station 8184 at Three Rivers, Mich., and station 4244 at Kalamazoo State Hospital, Kalamazoo, Mich. Water losses are the difference between precipitation and and total runoff and include evapotranspiration and soil moisture losses. Total runoff data are from records at U.S. Geological Survey gaging station 4-0971.7 on Portage River near Vicksburg, Mich. Surface runoff is the difference between total runoff and ground-water runoff. Change in storage is based on change in average water level in wells $3-10-31-1,4-10-17-1$, and 3-9-19-3. Surface drainage area is $68.2 \mathrm{sq} \mathrm{mi}$. Ground-water recharge per square mile: mgd, million gallons per day]

\begin{tabular}{|c|c|c|c|c|c|c|c|c|c|}
\hline & \multirow{2}{*}{$\begin{array}{l}\text { Preclpi- } \\
\text { tation }\end{array}$} & \multirow{2}{*}{$\begin{array}{l}\text { Water } \\
\text { losses }\end{array}$} & \multirow{2}{*}{$\begin{array}{l}\text { Total } \\
\text { runoff }\end{array}$} & \multirow{2}{*}{$\begin{array}{l}\text { Surface } \\
\text { runoff }\end{array}$} & \multicolumn{5}{|c|}{ Ground-water budget } \\
\hline . & & & & & Runoff & $\begin{array}{l}\text { Evapotrans- } \\
\text { píration }\end{array}$ & $\begin{array}{l}\text { Change in } \\
\text { storage }\end{array}$ & $\begin{array}{l}\text { Recharge } \\
\text { excluding } \\
\text { underfow }\end{array}$ & $\begin{array}{l}\text { Recharge } \\
\text { per sq mi } \\
\text { (mgd) }\end{array}$ \\
\hline \begin{tabular}{l}
\multicolumn{1}{c}{1864} \\
October.... \\
November... \\
December...
\end{tabular} & $\begin{array}{l}1.00 \\
2.27 \\
1.97\end{array}$ & $\begin{array}{l}0.79 \\
2.04 \\
1.67\end{array}$ & $\begin{array}{l}0.21 \\
.23 \\
.40\end{array}$ & $\begin{array}{l}0.01 \\
.02 \\
.04\end{array}$ & $\begin{array}{r}0.20 \\
.21 \\
.36\end{array}$ & $\begin{array}{l}0.14 \\
0.13 \\
\text { Negligible... }\end{array}$ & $\begin{array}{r}-0.53 \\
+.43 \\
+.33\end{array}$ & $\begin{array}{r}0.00 \\
.77 \\
.69\end{array}$ & $\begin{array}{r}0.00 \\
.43 \\
.39\end{array}$ \\
\hline Total. . & $\ldots$ & 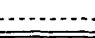 & -.... & (n.......... & $\ldots$ & (n) & (n) & $\ldots$ & - \\
\hline 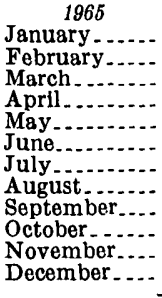 & $\begin{array}{l}3.90 \\
2.27 \\
3.08 \\
2.52 \\
1.83 \\
2.97 \\
2.03 \\
4.80 \\
5.16 \\
2.24 \\
2.52 \\
5.02\end{array}$ & $\begin{array}{l}3.28 \\
1.35 \\
1.71 \\
1.07 \\
1.17 \\
2.66 \\
1.82 \\
4.60 \\
4.80 \\
1.69 \\
1.96 \\
4.05\end{array}$ & $\begin{array}{l}.62 \\
.92 \\
1.37 \\
1.45 \\
.66 \\
.41 \\
.21 \\
.20 \\
.36 \\
.55 \\
.56 \\
.97\end{array}$ & $\begin{array}{l}.14 \\
.38 \\
.47 \\
.44 \\
.05 \\
.07 \\
.01 \\
.02 \\
.04 \\
.08 \\
.05 \\
.29\end{array}$ & $\begin{array}{l}.48 \\
.54 \\
.90 \\
1.01 \\
.61 \\
.34 \\
.20 \\
.18 \\
.32 \\
.47 \\
.51 \\
.68\end{array}$ & 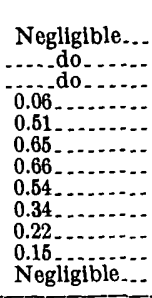 & $\begin{array}{r}+.62 \\
+.81 \\
+1.28 \\
+.99 \\
-.49 \\
-.62 \\
-.88 \\
-.42 \\
+.07 \\
+.04 \\
+.01 \\
+1.47 \\
\end{array}$ & $\begin{array}{l}1.10 \\
1.35 \\
2.18 \\
2.06 \\
.63 \\
.37 \\
.00 \\
.30 \\
.73 \\
.73 \\
.67 \\
2.15\end{array}$ & $\begin{array}{r}.62 \\
.84 \\
1.22 \\
1.19 \\
.35 \\
.21 \\
.00 \\
.17 \\
.42 \\
.41 \\
.39 \\
1.20\end{array}$ \\
\hline Total.. & 38.34 & 30.06 & 8.28 & 2.04 & 6.24 & $3.13 \ldots \ldots$ & +2.88 & 12.25 & $\ldots$ \\
\hline 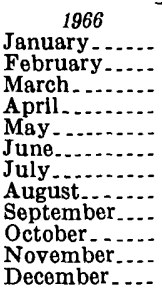 & $\begin{array}{l}.62 \\
1.46 \\
2.80 \\
4.68 \\
4.11 \\
2.45 \\
2.05 \\
4.75 \\
1.37 \\
1.30 \\
5.23 \\
3.77\end{array}$ & $\begin{array}{r}-.49 \\
.50 \\
1.47 \\
3.37 \\
2.31 \\
1.79 \\
1.70 \\
4.40 \\
.98 \\
.87 \\
4.53 \\
2.56\end{array}$ & $\begin{array}{l}1.11 \\
.96 \\
1.33 \\
1.31 \\
1.80 \\
.66 \\
.35 \\
.35 \\
.39 \\
.43 \\
.70 \\
1.21\end{array}$ & $\begin{array}{l}.21 \\
.12 \\
.32 \\
.33 \\
.70 \\
.04 \\
.01 \\
.04 \\
.01 \\
.02 \\
.11 \\
.32\end{array}$ & $\begin{array}{l}.90 \\
.84 \\
1.01 \\
.98 \\
1.10 \\
.62 \\
.34 \\
.31 \\
.38 \\
.41 \\
.59 \\
.89\end{array}$ & $\begin{array}{c}\text { Negligible... } \\
0.05 \\
0.14 \\
0.58 \\
0.68 \\
0.58 \\
0.41 \\
0.30 \\
0.08 \\
\text { Negligible.... }\end{array}$ & $\begin{array}{r}-.06 \\
+.37 \\
+.49 \\
+.63 \\
+.45 \\
-.69 \\
-.86 \\
-.46 \\
-.58 \\
-.37 \\
+.06 \\
+1.40\end{array}$ & $\begin{array}{l}.84 \\
1.21 \\
1.50 \\
1.66 \\
1.69 \\
.51 \\
.16 \\
.43 \\
.21 \\
.34 \\
.73 \\
2.29\end{array}$ & $\begin{array}{l}.47 \\
.75 \\
.84 \\
.96 \\
.95 \\
.30 \\
.09 \\
.24 \\
.12 \\
.19 \\
.42 \\
1.28\end{array}$ \\
\hline Total ..... & 34.59 & 23.99 & 10.60 & 2.23 & 8.37 & $2.82 \ldots \ldots$ & +.38 & 11.57 . & \\
\hline
\end{tabular}

mates of recharge in the Kalamazoo River basin for the period 1933-47 (table 6) were obtained from a relationship between groundwat er discharge and recharge based on data for the period 194862 (fig. 4). The relationship during the dry years 1963-64 and the normal years 1965 and 1966 are also shown. It should be pointed out that estimated records of ground-water recharge in tables 4,5 , and 6 are less accurate than those in tables 2 and 3 because groundwater levels and changes in storage had to be extended by correlation. However, the extended records are useful in arriving at an estimate of the long-term average rate of recharge.

Estimated ground-water recharge in the Kalamazoo River basin during the 34-year period 1933-66 varied markedly. It ranged from about 4 inches in 1964 to 13 inches in 1943 and 1950; the average was 
TABLE 4.-Kalamazoo River discharge and estimated rate of ground-water recharge in Kalamazoo River basin, 1948-62

\begin{tabular}{|c|c|c|c|c|c|c|c|}
\hline \multirow[b]{2}{*}{ Year } & \multirow{2}{*}{$\begin{array}{c}\text { Annuial } \\
\text { discharge, } \\
\text { Kalamazoo } \\
\text { River } \\
\text { (inches) }\end{array}$} & \multicolumn{6}{|c|}{ Ground-water budget } \\
\hline & & $\begin{array}{l}\text { Runoff } \\
\text { (Inches) }\end{array}$ & $\begin{array}{l}\text { Evapo- } \\
\text { transpi- } \\
\text { ration } \\
\text { (inches) }\end{array}$ & $\begin{array}{l}\text { Change } \\
\text { in storage } \\
\text { (inches) }\end{array}$ & $\begin{array}{l}\text { Recharge } \\
\text { (inches) }\end{array}$ & $\begin{array}{l}\text { Recharge } \\
\text { (inch } \\
\text { per day) }\end{array}$ & $\begin{array}{l}\text { Recharge } \\
\text { per sq } \\
\text { mi (mgd) }\end{array}$ \\
\hline $\begin{array}{l}1948 \\
19490 \\
1951 \\
1952 \\
1953 \\
1954 \\
1955 \\
1956 \\
1957 \\
1958 \\
1959 \\
1960 \\
1961 \\
1962\end{array}$ & $\begin{aligned} 13.51 \\
11.82 \\
18.09 \\
14.69 \\
14.07 \\
8.39 \\
11.22 \\
9.93 \\
10.85 \\
9.05 \\
7.41 \\
10.04 \\
12.19 \\
8.96 \\
8.68\end{aligned}$ & $\begin{array}{l}10.7 \\
10.2 \\
13.2 \\
10.2 \\
11.6 \\
8.2 \\
6.9 \\
8.6 \\
8.3 \\
6.9 \\
7.1 \\
7.0 \\
9.4 \\
7.9 \\
7.6\end{array}$ & $\begin{array}{r}0.8 \\
.9 \\
1.1 \\
.7 \\
.7 \\
.6 \\
.5 \\
.8 \\
1.4 \\
.5 \\
.6 \\
.4 \\
.7 \\
.8 \\
1.1\end{array}$ & $\begin{array}{r}-0.2 \\
+1.1 \\
-1.0 \\
+1.0 \\
-1.1 \\
-1.0 \\
+.8 \\
-.4 \\
-.7 \\
+.6 \\
-1.6 \\
+1.0 \\
-.4 \\
-.5 \\
-.6\end{array}$ & $\begin{array}{r}11.3 \\
12.2 \\
13.3 \\
11.9 \\
11.2 \\
7.8 \\
8.2 \\
9.0 \\
9.0 \\
8.0 \\
6.1 \\
8.4 \\
9.7 \\
8.1 \\
8.1\end{array}$ & $\begin{array}{l}0.031 \\
.033 \\
.036 \\
.033 \\
.031 \\
.022 \\
.022 \\
.025 \\
.025 \\
.022 \\
.017 \\
.023 \\
.027 \\
.022 \\
.022\end{array}$ & $\begin{array}{l}0.55 \\
.58 \\
.65 \\
.58 \\
.55 \\
.39 \\
.38 \\
.44 \\
.44 \\
.40 \\
.30 \\
.41 \\
.47 \\
.39 \\
.39\end{array}$ \\
\hline
\end{tabular}

TABLE 5.-Portage River discharge and estimated rate of ground-water recharge in St. Joseph River basin, 1947-51

\begin{tabular}{|c|c|c|c|c|c|c|c|}
\hline \multirow[b]{2}{*}{ Year } & \multirow{2}{*}{$\begin{array}{l}\text { Annual } \\
\text { discharge } \\
\text { Portage } \\
\text { River } \\
\text { (inches) }\end{array}$} & \multicolumn{6}{|c|}{ Ground-water budget } \\
\hline & & $\begin{array}{c}\text { Runoff } \\
\text { (inches) }\end{array}$ & $\begin{array}{l}\text { Evapo- } \\
\text { transpi- } \\
\text { ration } \\
\text { (inches) }\end{array}$ & $\begin{array}{l}\text { Change } \\
\text { in storage } \\
\text { (inches) }\end{array}$ & $\begin{array}{l}\text { Recharge } \\
\text { (inches) }\end{array}$ & $\begin{array}{l}\text { Recharge } \\
\text { (inch } \\
\text { per day) }\end{array}$ & $\begin{array}{l}\text { Recharge } \\
\text { per sq } \\
\text { mi (mgd) }\end{array}$ \\
\hline $\begin{array}{l}1947 \\
1948 \\
19490 \\
1951\end{array}$ & $\begin{array}{l}14.0 \\
12.6 \\
11.6 \\
17.7 \\
12.6\end{array}$ & $\begin{array}{r}8.8 \\
7.5 \\
7.6 \\
12.3 \\
9.6\end{array}$ & $\begin{array}{l}1.7 \\
.7 \\
1.1 \\
3.2 \\
1.8\end{array}$ & $\begin{array}{r}-0.7 \\
-.3 \\
+1.4 \\
-1.3 \\
+1.3\end{array}$ & $\begin{array}{r}9.8 \\
7.9 \\
10.1 \\
14.2 \\
12.7\end{array}$ & $\begin{array}{l}0.027 \\
.022 \\
.028 \\
.039 \\
.035\end{array}$ & $\begin{array}{l}0.47 \\
.38 \\
.48 \\
.68 \\
.60\end{array}$ \\
\hline
\end{tabular}

TABLE 6.-Kalamazoo River discharge and extended estimates of ground-water recharge in Kalamazoo River basin, 1993-47

[Taken from relations shown in fig. 4]

\begin{tabular}{|c|c|c|c|c|}
\hline \multirow{2}{*}{ Year } & \multirow{2}{*}{$\begin{array}{l}\text { Annual dis- } \\
\text { charge, Kala- } \\
\text { mazoo River } \\
\text { (inches) }\end{array}$} & \multicolumn{3}{|c|}{$\begin{array}{c}\text { Estimated ground-water recharge, } \\
\text { Kalamaz0o River basin }\end{array}$} \\
\hline & & Inches & $\begin{array}{l}\text { Inch per } \\
\text { day }\end{array}$ & $\underset{\mathrm{mi}}{\mathrm{Mgd}} \mathbf{p e r} \mathrm{sq}$ \\
\hline $\begin{array}{l}1933 \\
1934 \\
1935 \\
1936 \\
1937 \\
1938 \\
1939 \\
1940 \\
1941 \\
1942 \\
1943 \\
1944 \\
1945 \\
1946 \\
1947\end{array}$ & $\begin{array}{r}11.15 \\
16.45 \\
28.80 \\
7.93 \\
11.69 \\
10.63 \\
9.45 \\
9.57 \\
10.15 \\
13.99 \\
18.27 \\
11.47 \\
10.62 \\
8.99 \\
15.36\end{array}$ & $\begin{array}{r}9.5 \\
5.3 \\
7.6 \\
6.9 \\
9.7 \\
9.0 \\
8.1 \\
8.2 \\
8.8 \\
11.1 \\
13.2 \\
9.7 \\
9.0 \\
7.8 \\
11.9\end{array}$ & $\begin{array}{l}0.026 \\
.014 \\
.021 \\
.019 \\
.027 \\
.025 \\
.022 \\
.022 \\
.024 \\
.030 \\
.036 \\
.027 \\
.025 \\
.022 \\
.033\end{array}$ & $\begin{array}{r}0.45 \\
.24 \\
.36 \\
.33 \\
.47 \\
.44 \\
.39 \\
.38 \\
.42 \\
.52 \\
.65 \\
.47 \\
.44 \\
.39 \\
.58\end{array}$ \\
\hline
\end{tabular}

1 Estimated.

2 Adjusted. 


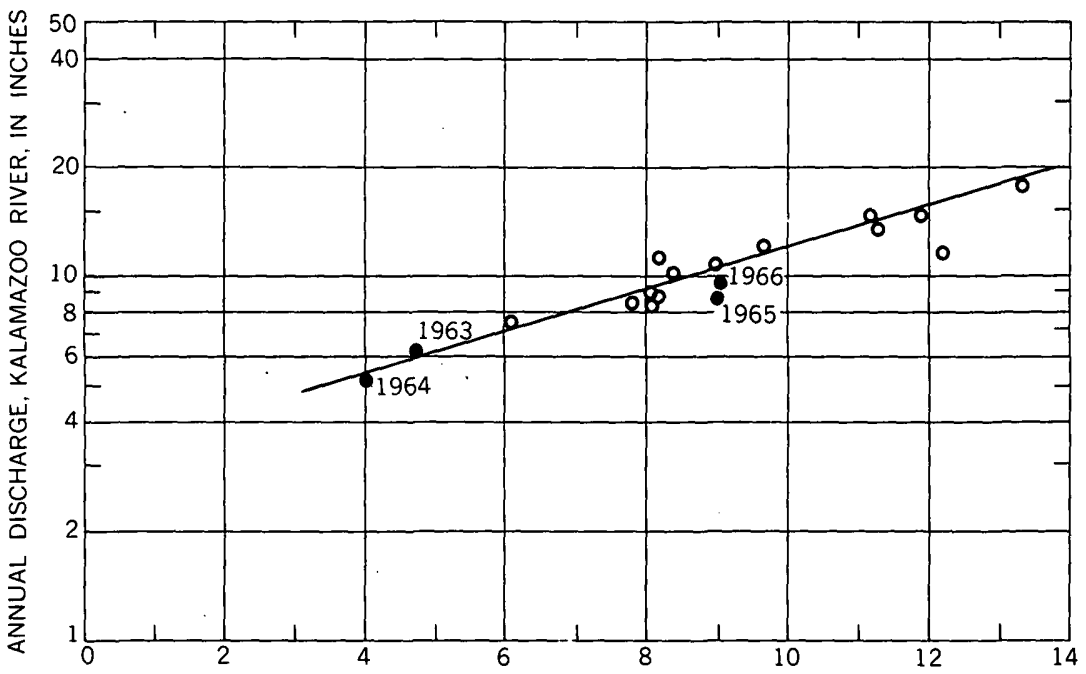

ANNUAL GROUND.WATER RECHARGE, KALAMAZOO RIVER BASIN, IN INCHES

Figure 4.-Relationship of total runoff to ground-water recharge in Kalamazoo River basin, 1948-62. Data for 1963-66 are shown for comparison.

about 9 inches. The long-term average rate of recharge in the St. Joseph River basin was determined by comparing concurrent discharge records in the Kalamazoo and St. Joseph basins. The recharge rates are shown in the following table:

Comparison of annual recharge rates

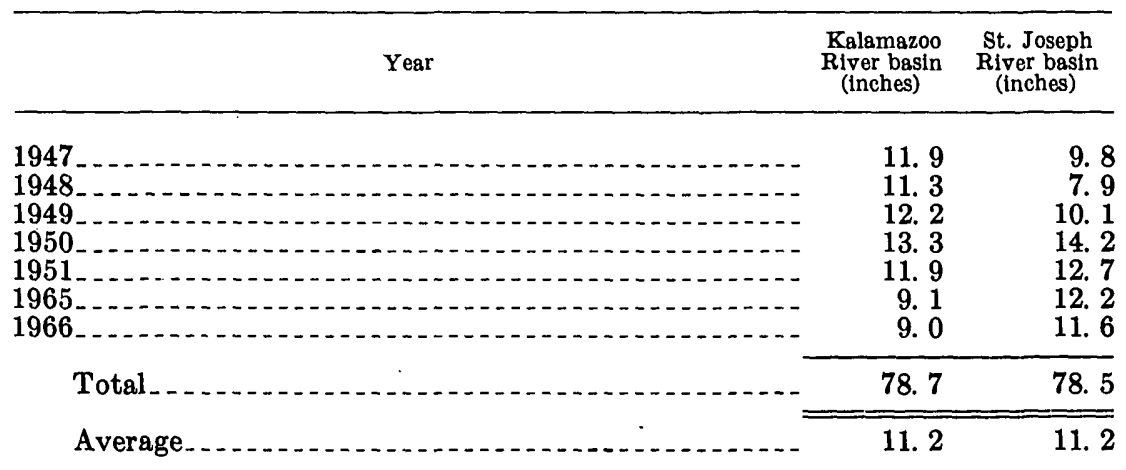

Since the average (normal) rate of recharge for the two basins is about the same, it is assumed that the long-term average recharge of about 9 inches for the Kalamazoo River basin is also representative of the long-term average for the St. Joseph River basin. The annual recharge rate of 9 inches for both basins is used in a later section of 
this report to compute the potential yield of the major ground-water reservoirs.

\section{NATURAL DISCHARGE AND MOVEMENT OF GROUND WATER}

Natural discharge from the ground-water system is by seepage to springs, streams, and some lakes and to a lesser extent by evapotranspiration from the water table. The hydraulic gradient in Kalamazoo County can be determined from contours on the water table (pl. 4). The configuration of the water table in Kalamazoo County shows that ground water moves from topographically high areas to discharge into ponds, streams, marshes, and other lowland areas where the water table is at or near the land surface. Several of the larger lakes, such as Austin, West, and Long Lakes, are near ground-water divides, and water moves by underflow both into and out of these lakes.

Water-level measurements in observation wells and measurements made in a large number of additional wells were used in drawing the contours on the water table. The contours represent the approximate position of the water table in November 1964 at a time when water levels were exceptionally low. Because water levels in all wells were not measured in November 1964, it was necessary to estimate the level of the water in some of the wells by correlation of the water-level records of these wells with the records of the wells that were measured in November. At many wells, altitudes of land surface used in computing water-level altitudes had to be estimated to the nearest 10 -feet from available topographic maps. In the vicinity of Austin Lake in the south-central part of the county, water-level altitudes were accurately determined by leveling, and a 5-foot water-table contour interval was used. A more detailed discussion of groundwater movement in that area is included in the section on lakes.

The low flow of streams in Kalamazoo County, shown on plate 4, are based on the lowest flows measured during three seepage runs. A seepage run involves making a number of streamflow measurements during a period of dry weather at many sites to show base-flow conditions. The results depict flow conditions on July 27 and 28, 1964. Streamflow at that time was about what could be expected to be equaled or exceeded 95 percent of the time.

Records of streamflow obtained in seepage runs, together with data on the slope of the water table, can be used to estimate the transmissibility of the glacial materials in the local drainage area. If the slopes of the water table toward the streams are about the same, it can be assumed that a large increase in flow per unit area of discharge generally indicates glacial materials with high transmissibility; a 
small increase in flow per unit of drainage area indicates glacial materials of low transmissibility. The use of low-flow data as an index to transmissibility can, however, be misleading in that an aquifer mantled by an aquiclude generally does not contribute significantly to the dry-weather flow of streams. Thus, streams with a low base flow may be underlain by aquicludes which in turn are underlain by productive aquifers.

The upper aquifer in Kalamazoo County indicates in a general way where the variations in low flow can be related to the saturated thickness of the upper aquifer (pl. 4). For example, in the southeastern quarter of the county, the upper aquifer is missing or relatively thin. Streams such as Bear Creek, Little Portage Creek, and Portage River draining this area generally have a smaller low-flow discharge than streams such as Gourdneck Creek and Portage Creek below Vicksburg draining an equivalent area in the southwest quarter of the county where the upper aquifer is relatively thick.

Other interesting hydrologic features are shown on plate 4. For example, the low-flow data show large pickup or loss in flow in some streams within relatively short distances. The increases result from a large discharge of ground water where the upper aquifer is highly permeable. Water is lost from the stream near well fields or where water moves from one subbasin to another. Losses of these kinds occur on the West Fork Portage Creek, in the west-central part of the county. Two seepage runs during 1964 and 1965 show losses of 1.30 and 0.72 cfs (cubic feet per second), respectively, between 12th Street and the gaging station at Oakland Drive. The loss below 12th Street, southwest of Oakwood Heights, is probably caused by pumping at the city of Kalamazoo well field at Station 22 located between Atwater Pond and gaging station 1064.0 at Oakland Drive (pl. 8). The well field is approximately half a mile from the creek. During the period 1964-66, an average of $620,000,000$ gallons per year was pumped from the field. Two seepage runs made in the West Fork Portage Creek basin in 1959, prior to the development of well field 22, indicated little or no loss in flow between Atwater Pond and Oakland Drive (Reed and others, 1966). Significant losses, however, did occur from Oakland Drive to the mouth of the stream. The losses below Oakland Drive are the result of induced recharge caused by pumping from the city of Kalamazoo well field at Station 9 (pl. 8). Losses due to pumpage are still evident in this reach of the stream.

Upstream from 12th Street the West Fork Portage Creek watershed contains many lakes and ponds. In this reach of the stream, water seeps to the water table and then moves as underflow to Portage Creek. The water-table contours on plate 4 indicate that 
ground water moves in a southeast direction from West Fork Portage Creek to Portage Creek near Portage, resulting in higher runoff per unit of surface drainage area for Portage Creek. A comparison of annual runoff figures for these two streams is shown below:

\begin{tabular}{|c|c|c|c|c|c|c|}
\hline & \multicolumn{3}{|c|}{$\begin{array}{l}\text { West Fork Portage Creek, station } 1064.00 \\
\text { (drainage area } 18.7 \mathrm{sq} \mathrm{mi} \text { ) }\end{array}$} & \multicolumn{3}{|c|}{$\begin{array}{l}\text { Portage Creek near Portage, station } 1061.90 \\
\text { (drainage area 18.6 sq mi) }\end{array}$} \\
\hline & Mean (cfs) & Cfs per sq mi & Inches & Mean (cfs) & Cfs per sq mi & Inches \\
\hline 196 & 4. 85 & 0. 259 & & 18. 9 & 1. 02 & 13. \\
\hline $1966 \ldots$ & 6. 18 & .330 & 4. 48 & 21. 7 & 1. 17 & 15. 8 \\
\hline $1967 \ldots \ldots$ & 10. 20 & .545 & 7. 42 & 26. 5 & 1. 42 & 19. 3 \\
\hline
\end{tabular}

In the summer, water temperatures for Portage Creek are as much as $7.8^{\circ} \mathrm{C}\left(14^{\circ} \mathrm{F}\right)$ colder than those for West Fork Portage Creek. Conversely, during the winter, water temperatures for Portage Creek are as much as $5.5^{\circ} \mathrm{C}\left(10^{\circ} \mathrm{F}\right)$ warmer than West Fork Portage Creek. This reveals that Portage Creek is getting a larger ground-water contribution than West Fork Portage Creek.

Spring Brook, a tributary to the Kalamazoo River about 5 miles north of Kalamazoo, shows large ground-water contribution throughout its length. This stream was measured at three sites during each seepage run (pl. 5). The results are shown in the following table:

\begin{tabular}{|c|c|c|c|c|c|c|c|c|}
\hline \multirow{2}{*}{ Site } & \multirow{2}{*}{ Station } & \multirow{2}{*}{$\begin{array}{l}\text { Drain- } \\
\text { age } \\
\text { area } \\
\text { (sq mi) }\end{array}$} & \multicolumn{2}{|c|}{ July 1964} & \multicolumn{2}{|c|}{ October 1965} & \multicolumn{2}{|c|}{ May 1967} \\
\hline & & & Cfs & $\begin{array}{l}\text { Cfs per } \\
\text { sq mi }\end{array}$ & Cfs & $\begin{array}{l}\text { Cfs per } \\
\text { sq mi }\end{array}$ & Cfs & $\begin{array}{l}\text { Cfs per } \\
\text { sq mi }\end{array}$ \\
\hline $\begin{array}{l}\text { 27th Street. } \\
\text { CD Avenue } \\
\text { Riverview Road. }\end{array}$ & $\begin{array}{l}1067.20 \\
1067.30 \\
1067.50\end{array}$ & $\begin{array}{l}20.5 \\
22.4 \\
31.1\end{array}$ & $\begin{array}{l}0.58 \\
\text { 3. } 42 \\
9.70\end{array}$ & $\begin{array}{r}0.028 \\
.153 \\
.312\end{array}$ & $\begin{array}{r}0.70 \\
5.07 \\
12.3\end{array}$ & $\begin{array}{r}0.034 \\
.226 \\
.395\end{array}$ & $\begin{array}{l}1.94 \\
8.55 \\
19.2\end{array}$ & $\begin{array}{r}0.095 \\
.382 \\
.617\end{array}$ \\
\hline
\end{tabular}

In each run, the increase in discharge between sites was significant. During the July 1964 run, the intervening drainage area between 27th Street and CD Avenue (1.9 sq mi) had a yield of $1.34 \mathrm{cfs}$ per sq mi and the intervening area between $\mathrm{CD}$ Avenue and Riverview Rd. (8.7 sq mi) had a yield of $0.76 \mathrm{cfs}$ per sq mi. An examination of plate 4 shows that Spring Brook has cut through the upper aquifer and is receiving seepage from the entire saturated thickness, or as much as 60 feet.

Other small areas which have large yields per unit area are as follows: the area draining into Rupert Lake in the northwest corner of the county, the area between stations 1056.80 and 1057.00 on Augusta Creek, the area between stations 970.80 and 971.20 on Portage River, and the area between stations 973.55 and 973.70 on Flowerfield Creek (pl. 5). 
Water is also discharged directly from the upper aquifer by evapotranspiration. The rate of evapotranspiration is dependent upon climatic factors, depth to water table, and type of vegetation. In Kalamazoo County evapotranspiration occurs chiefly from May through September (tables 2 and 3). When ground-water discharge exceeds recharge, ground-water runoff diminishes, water levels decline, and streamflows decrease except when local precipitation is sufficient to produce surface runoff. A decrease in the rate of evapotranspiration in August, September, and October can cause groundwater runoff to increase even though ground-water levels are declining (pl. 3).

\section{CHEMICAL AND PHYSICAL QUALITY OF WATER}

The chemical, physical, and biological quality of water determines its usefulness for agriculture, industry, and municipal supply. Chemical quality refers to type and amount of dissolved minerals present, whereas physical quality refers to temperature, color, suspended solids, and other physical properties of water. Biological quality considers species and number of living organisms and their interaction with the environment. The types of water quality are not fully independent of each other but have traditionally been treated separately. Only the chemical and physical quality of water is dealt with in this report.

Chemical constituents that compose most of the dissolved solids in the water of Kalamazoo County are the following six ions; calcium $\left(\mathrm{Ca}^{+2}\right)$, magnesium $\left(\mathrm{Mg}^{+2}\right)$, sodium $\left(\mathrm{Na}^{+}\right)$, bicarbonate $\left(\mathrm{HCO}_{3}{ }^{-}\right)$, sulfate $\left(\mathrm{SO}_{4}{ }^{-2}\right)$, and chloride $\left(\mathrm{Cl}^{-}\right)$. Iron, which restricts the use of water for many applications, is present in small but important amounts. Analytical procedures used by the U.S. Geological Survey are given by Rainwater and Thatcher (1960). Analyses performed by the Michigan Department of Health follow standard procedures suggested by the American Public Health Association, American Water Works Association, and Water Pollution Control Federation (1965).

Table 7 indicates the source or cause of the major chemical constituents and physical properties of water in Kalamazoo County and describes their effects on the utility of the water for various uses. Table 8 lists representative analyses of ground water in the county. Analyses of surface water are included in the section on availability of water.

\section{TEMPERATURE OF GROUND WATER}

One important physical property of ground water is its temperature. Because ground-water temperatures normally fluctuate only 
TABLE 7.-Source and significance of chemical constituents and physical properties commonly occurring in natural surface and ground water

[USPHS, U.S. Public Health Service; mg/, milligram per liter]

\begin{tabular}{lll}
\hline Constituent or property & Source or cause & Significance \\
\hline
\end{tabular}

Silica $\left(\mathrm{SiO}_{2}\right)$............. Dissolved from nearly all rocks and Contributes to formation of boiler scale. soils.

Iron $(\mathrm{Fe})$.............. Dissolved from the common ironbearing minerals present in most formations.

Manganese (Mn)

Dissolved from manganese-bearing minerals. Present in most acid waters.

Calcium (Ca) and magnesium $(\mathrm{Mg})$.

Sodium (Na) and potassium (K).

Bicarbonate $\left(\mathrm{HCO}_{3}\right)$ and carbonate $\left(\mathrm{CO}_{8}\right)$.

Sulfate $\left(\mathrm{SO}_{4}\right)$

Dissolved from shales and gypsum. Oxidation of sulfides. Commonly associated with coal-mining operations. Contributed by some industrial wastes

Chloride (Cl)

Dissolved in varying amounts in all solls and rocks. Also found in brines, sea water, and sewage.

Fluoride (F).

Small amount available from most rocks and soils. Most fluoride concentrations over $1 \mathrm{mg} / 1$ usually found in sodium waters. Primary source of high concentration is industrial pollution. Added to many municipal supplies by fluoridation.

Nitrate $\left(\mathrm{NO}_{3}\right)$. .......... Decaying organic matter. Nitrate fertllizers. Sewage.

Phosphate (PO)

Dissolved from many rocks and solls. The orthophosphate form is the only form derived from natural sources. Other forms as well as orthophosphate come from fertilizers, detergents, domestic and industrial wastes. Small amounts of polyphosphate are used in some water treatment plants for softening.
Chiefly mineral constituents. dis-

Dissolved solids solved from rocks and soils. Often includes some water of crystallization.
Inhibits deterioration of zeolite-type water softeners.

Oxidizes to a reddish-brown sediment. Stains utensils, enamelware, clothing, and other articles. Unsatisfactory for food processing, dyeing, laundering, bleaching, beverages, textiles, processIng of ice. USPHS (1962) drinking water standards suggest that iron should not exceed $0.3 \mathrm{mg} / 1$.

Same objectionable features as iron. Causes black-brown stain. USPHS (1962) drinking water standards suggest that manganese should not exceed 0.05 $\mathrm{mg} / \mathrm{l}$.

Impart hardness and scale-forming properties to water; are soap consuming (see hardness). Unsuitable for laundries, steam plants, textile processing, and dyeing.

Cause boller foaming when present in large amounts. Combine with chloride to give a salty taste. Large quantities may limit use for irrigation.

Raise the alkalinity and usually $\mathrm{pH}$ of water. In combination with calcium and magnesium, cause carbonate hardness and scale. Release corrosive carbon dioxide gas on heating.

With calcium, forms hard scale in steam boilers. Imparts cloudiness to ice. Causes bitter taste when combined in large amounts with other lons. Calcium sulfate considered beneficial in brewing processes. USPHS (1962) drinking water standards recommend that sulfate content not exceed $250 \mathrm{mg} / 1$.

Calcium and magnesium chloride may hydrolyze and increase the corrosive activity of water. In large amounts gives salty taste. USPHS (1962) drinking water standards recommend that chloride content should not exceed 250 $\mathrm{mg} / \mathrm{l}$.

May cause mottling of enamel on teeth of children if present in amounts in excess of about $1.5 \mathrm{mg} / 1$. A bout $1 \mathrm{mg} / \mathrm{l}$ reduces incidence of tooth decay in chlldren (Maier, 1950). USPHS recommends control limits based upon annual average of maximum daily air temperatures. (See USPHS, 1962, p. 8.)

Investigations by Comly (1945) indicate that high concentrations (more than 44 $\mathrm{mg} / \mathrm{l}$ expressed as $\mathrm{NO}_{3}$ ) may cause methemoglobinemia (infant cyanosis). USPHS (1962) drinking water standards suggest a limit of $45 \mathrm{mg} / \mathrm{l}$. Enards suggest a limit of $45 \mathrm{mg} / \mathrm{l}$. Enorganisms which produce undisirable tastes and odors. Higher than local average may suggest pollution.

Generally, concentrations encountered in water are not toxic to man, animals, or fish. Phosphates stimulate the growth of algae which may cause odor and (or) taste problems in water supplies. Algae may also give water an unsightly appearance.

USPHS (1962) drinking water standards recommend that the dissolved solids should not exceed $500 \mathrm{mg} /$. However, $1,000 \mathrm{mg} / 1$ is permitted under certsin circumstances. Waters containing more than $1,000 \mathrm{mg} / \mathrm{l}$ of dissolved solids are unsuitable for many purposes. 
TABLE 7.-Source and significance of chemical constituents and physical properties commonly occurring in natural surface and ground water-Continued

\begin{tabular}{lll}
\hline Constituent or property & Source or cause & Signifleance
\end{tabular}

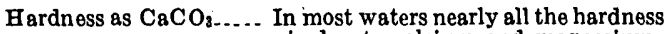
is due to calcium and magnesium. All the metallic cations other than the alkall metals also cause hardness.

Specific conductance (micromhos at $25^{\circ} \mathrm{C}$ ).

Dissolved mineral content of the water.

Consumes soap before a lather will form. Deposits soap curd on bathtubs. Hard water forms scale in boilers, water heaters, and pipes. Hardness equivalent to the bicarbonate and carbonate is called carbonate hardness. Any hardness in excess of this is called noncarbonate hardness. Waters of hardness up to $60 \mathrm{mg} / \mathrm{l}$ are considered soft; 61 to 120 $\mathrm{mg} / \mathrm{l}$, moderately hard; 121 to $180 \mathrm{mg} /$, hard; more than $180 \mathrm{mg} / \mathrm{l}$, very hard (U.S. Geological Survey).

pH (hydrogen-ion con-
centration or centration or
activity).

Acids, acid-generating salts, and free carbon dloxide lower the $p H$. Car bonates, bicarbonates, hydroxides, phosphates, silicates, and borates generally raise the $\mathrm{pH}$.

Color

Yellow-to-brown color of some waters is usually caused by organic matter extracted from leaves, roots, and other organic substances. Color in water also results from industrial wastes and sewage.

Tomperature cooling agent, industrial pollution.

Dissolved oxygen

Plants produce oxygen in their growth processes. Oxygen is consumed in the decay processes of organic material.
Indicates degree of mineralization. Is a measure of the capacity of the water to conduct an electric current. Varies with concentration and degree of Ionization of the constituents.

A pH of 7.0 indicates neutrality of a solution. Values higher than 7.0 denote increasing alkalinity; values lower than 7.0 indicate increasing acidity. $\mathrm{pH}$ is a measure of the activity of the hydrogen ions. Corrosiveness of water generally increases with decreasing $\mathrm{pH}$. However, excessively alkaline wators may also attack metals.

Water for domestic use and for some industrial uses should be free from perceptible color. Color in water is objectionable in food and beverage processing and many manufacturing processes.

Affects usefulness of water for many purposes. For most uses, a water of uniformly low temperature is desired. Shallow wells show some seasonal fluctuations in water temperature. Ground waters from moderate depths usually are nearly constant in temperature, which is near the mean annual alr temperature of the area. In very deep wells, the water temperature generally increases on the average about $1^{\circ} \mathrm{F}$ with each 60 -foot increment of depth. Seasonal fluctuations in temperatures of surface waters are comparatively large depending on the volume of water.

Low percentage of saturation indicate the presence of oxygen demanding subtances. This condition is usually associated with pollution. Dissolved oxygen should be present in surface water to prevent the formation of anaerobic bacteria.

slightly, industrial processes that use ground water can be standardized without adjustment for temperature change. When the water is used for cooling, a Btu exchange rate can be relied upon without concern for seasonal changes in temperature.

The temperature of ground water was measured periodically in several wells by means of "lazy" thermometers (Heath, 1964). "Lazy" thermometers were used in order to reduce the expected difference between temperature of air and water. Graphs of water temperatures in three wells of different depths, unaffected by pumping, are shown in figure 5. Variation in annual temperature decreases with depth (fig. 6). For example, a well 15 feet deep would be expected to vary 
TABLE 8.-Selected chemical analyses

[Chemical analyses in milligrams per liter, except $\mathbf{p H}$, color, or where indicated otherwise. Analyst: USGS' and others (1960) and Michigan Department of Health (1961). 280 additional unpublished field analyses

\begin{tabular}{|c|c|c|c|c|c|c|c|c|}
\hline Well location & $\begin{array}{l}\text { Depth } \\
\text { of well } \\
\text { in feet }\end{array}$ & $\begin{array}{l}\text { Date } \\
\text { of col- } \\
\text { lection }\end{array}$ & $\begin{array}{r}\text { Silica } \\
\left(\mathrm{SiO}_{2}\right)\end{array}$ & $\begin{array}{l}\text { Iron } \\
\left(\mathrm{F}_{\theta}\right)\end{array}$ & $\begin{array}{c}\text { Cal- } \\
\text { clum } \\
(\mathrm{Ca})\end{array}$ & $\begin{array}{c}\text { Mag- } \\
\text { nesium } \\
(\mathbf{M g})\end{array}$ & $\begin{array}{l}\text { So- } \\
\text { dium } \\
\text { (Na) }\end{array}$ & $\begin{array}{l}\text { Potas- } \\
\text { slum } \\
\text { (K) }\end{array}$ \\
\hline
\end{tabular}

\begin{tabular}{|c|c|c|c|c|c|c|c|c|}
\hline 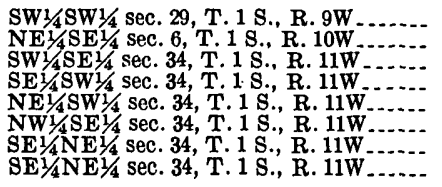 & $\begin{array}{l}99 \\
72 \\
52 \\
51 \\
46 \\
51 \\
46 \\
60\end{array}$ & $\begin{array}{r}10-26-66 \\
4-21-65 \\
6-14-62 \\
6-14-62 \\
6-14-62 \\
6-14-62 \\
9-11-64 \\
9-11-64\end{array}$ & $\begin{array}{l}11 \\
13 \\
12 \\
11 \\
12 \\
12 \\
12\end{array}$ & $\begin{array}{l}0.39 \\
.83 \\
.50 \\
.00 \\
.00 \\
.70 \\
.60 \\
.60\end{array}$ & $\begin{array}{r}63 \\
140 \\
84 \\
82 \\
74 \\
74 \\
58\end{array}$ & $\begin{array}{l}18 \\
-34 \\
32 \\
28 \\
30 \\
27 \\
21\end{array}$ & $\begin{array}{l}3.6 \\
20 \\
6.0 \\
5.5 \\
9.5 \\
23 \\
21\end{array}$ & $\begin{array}{r}1.8 \\
1.2 \\
.8 \\
1.0 \\
1.2 \\
1.2\end{array}$ \\
\hline 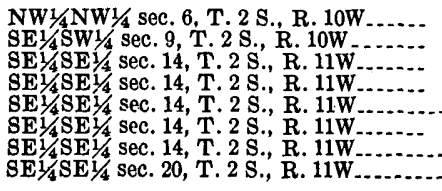 & $\begin{array}{l}102 \\
100 \\
100 \\
100 \\
98\end{array}$ & $\begin{array}{l}7-13-65 \\
7-14-65 \\
3-1-67 \\
4-27-67 \\
4-27-67 \\
4-27-67 \\
4-27-67 \\
8-10-66\end{array}$ & $\begin{array}{c}10 \\
9.0 \\
10 \\
7.0 \\
10 \\
13\end{array}$ & $\begin{array}{l}.30 \\
.90 \\
.40 \\
.10 \\
.00 \\
.70 \\
.50 \\
.90\end{array}$ & $\begin{array}{r}160 \\
120 \\
148 \\
138 \\
138 \\
68\end{array}$ & $\begin{array}{l}35 \\
30 \\
35 \\
35 \\
32 \\
27\end{array}$ & $\begin{array}{c}16 \\
20 \\
16 \\
23 \\
23 \\
6.0\end{array}$ & $\begin{array}{r}1.6 \\
1.5 \\
1.2 \\
2.0 \\
2.2 \\
.7\end{array}$ \\
\hline 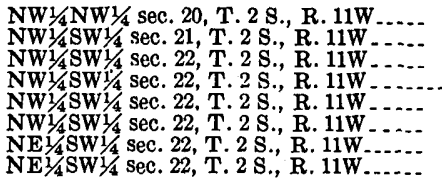 & $\begin{array}{l}149 \\
165 \\
151 \\
153 \\
139 \\
120 \\
165\end{array}$ & $\begin{array}{r}2-20-66 \\
10--62 \\
12-12-66 \\
12-12-66 \\
5-11-67 \\
5-11-67 \\
7-18-66 \\
4-4-67\end{array}$ & $\begin{array}{l}13 \\
15 \\
12 \\
10 \\
11 \\
13 \\
14 \\
15\end{array}$ & $\begin{array}{l}1.3 \\
.30 \\
1.0 \\
1.3 \\
.10 \\
2.1 \\
1.8 \\
2.6\end{array}$ & $\begin{array}{r}74 \\
64 \\
118 \\
100 \\
90 \\
94 \\
98 \\
144\end{array}$ & $\begin{array}{l}24 \\
22 \\
33 \\
32 \\
32 \\
33 \\
33 \\
41\end{array}$ & $\begin{array}{l}3.9 \\
4.6 \\
21 \\
18 \\
12 \\
24 \\
23 \\
16\end{array}$ & $\begin{array}{l}.6 \\
.8 \\
1.7 \\
1.7 \\
1.7 \\
1.6 \\
2.0 \\
1.6\end{array}$ \\
\hline 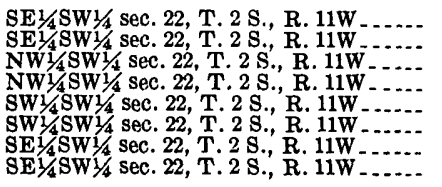 & $\begin{array}{l}142 \\
142 \\
146 \\
192 \\
132 \\
161 \\
150 \\
181\end{array}$ & $\begin{array}{l}3-27-67 \\
5-11-67 \\
5-11-67 \\
3-27-67 \\
3-27-67 \\
3-27-67 \\
4-5-67 \\
4-4-67\end{array}$ & $\begin{array}{l}12 \\
11 \\
12 \\
12 \\
13 \\
12 \\
13 \\
12\end{array}$ & $\begin{array}{l}.00 \\
.10 \\
.10 \\
1.4 \\
1.1 \\
1.2 \\
1.4 \\
1.0\end{array}$ & $\begin{array}{r}108 \\
74 \\
86 \\
90 \\
82 \\
82 \\
104 \\
102\end{array}$ & $\begin{array}{l}35 \\
27 \\
29 \\
28 \\
27 \\
25 \\
34 \\
32\end{array}$ & $\begin{array}{l}19 \\
12 \\
22 \\
15 \\
11 \\
11 \\
14 \\
29\end{array}$ & $\begin{array}{l}2.0 \\
1.4 \\
1.4 \\
1.4 \\
1.1 \\
1.0 \\
1.1 \\
2.4\end{array}$ \\
\hline 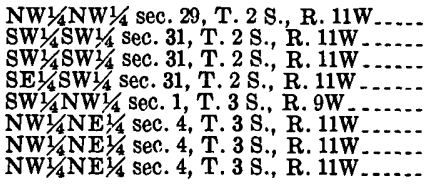 & $\begin{array}{l}179 \\
236 \\
216 \\
215 \\
111 \\
162 \\
163 \\
162\end{array}$ & $\begin{array}{r}9-28-62 \\
10-14-65 \\
10-14-65 \\
10-14-65 \\
8-31-66 \\
6-13-66 \\
6-12-67 \\
6-13-67\end{array}$ & $\begin{array}{l}17 \\
12 \\
12 \\
12 \\
17 \\
9.0 \\
10 \\
10\end{array}$ & $\begin{array}{l}.50 \\
.10 \\
.50 \\
.90 \\
.30 \\
.20 \\
.30\end{array}$ & $\begin{array}{l}58 \\
60 \\
64 \\
58 \\
59 \\
68 \\
58 \\
66\end{array}$ & $\begin{array}{l}22 \\
24 \\
22 \\
22 \\
19 \\
16 \\
19 \\
21\end{array}$ & $\begin{array}{l}5.3 \\
3.7 \\
4.6 \\
4.6 \\
6.7 \\
4.6 \\
3.2 \\
3.0\end{array}$ & $\begin{array}{r}.8 \\
.7 \\
.6 \\
.6 \\
1.0 \\
.7 \\
.9 \\
.6\end{array}$ \\
\hline 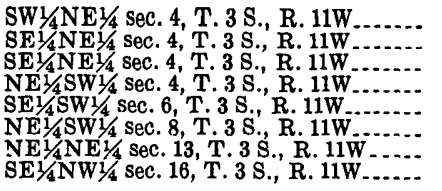 & $\begin{array}{l}166 \\
161 \\
162 \\
182 \\
185 \\
142 \\
174 \\
114\end{array}$ & $\begin{array}{l}6-12-67 \\
6-13-67 \\
6-13-67 \\
6-16-60 \\
6-10-61 \\
1-26-60 \\
2-20-62 \\
7-10-61\end{array}$ & $\begin{array}{l}11 \\
11 \\
10 \\
14 \\
13 \\
12 \\
11 \\
13\end{array}$ & $\begin{array}{l}.30 \\
.50 \\
.30 \\
.20 \\
.40 \\
.00 \\
.40 \\
.30\end{array}$ & $\begin{array}{l}70 \\
70 \\
72 \\
60 \\
56 \\
60 \\
66 \\
58\end{array}$ & $\begin{array}{l}26 \\
22 \\
22 \\
22 \\
18 \\
21 \\
21 \\
20\end{array}$ & $\begin{array}{l}3.2 \\
3.2 \\
3.7 \\
5.0 \\
4.6 \\
2.3 \\
2.3 \\
3.4\end{array}$ & $\begin{array}{r}1.0 \\
.6 \\
.5 \\
.8 \\
.8 \\
.4 \\
.4 \\
.8\end{array}$ \\
\hline 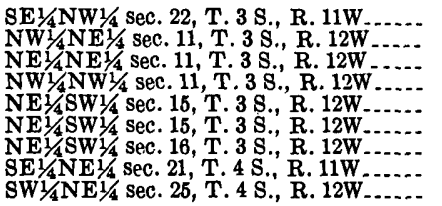 & $\begin{array}{l}120 \\
300 \\
270 \\
306 \\
183 \\
183 \\
197 \\
220 \\
280\end{array}$ & $\begin{array}{r}1-26-60 \\
9-7-61 \\
11-9-61 \\
1-18-60 \\
7-9-66 \\
4-3-67 \\
7-9-66 \\
11-28-66 \\
1-9-67\end{array}$ & $\begin{array}{l}11 \\
14 \\
16 \\
23 \\
14 \\
14 \\
15\end{array}$ & $\begin{array}{l}1.5 \\
.70 \\
1.2 \\
.60 \\
.60 \\
1.5 \\
0\end{array}$ & $\begin{array}{l}47 \\
58 \\
60 \\
36 \\
48 \\
47 \\
47 \\
46 \\
46\end{array}$ & $\begin{array}{l}12 \\
20 \\
21 \\
21 \\
12 \\
13 \\
11 \\
22 \\
33\end{array}$ & $\begin{array}{l}5.8 \\
5.8 \\
7.0 \\
5.6 \\
5.9 \\
3.7 \\
3.2 \\
4.4 \\
4.7\end{array}$ & $\begin{array}{l}.8 \\
.4 \\
.8 \\
.3 \\
.8 \\
.7 \\
.5 \\
4\end{array}$ \\
\hline
\end{tabular}

$3.8^{\circ} \mathrm{C}$ or $6.5^{\circ} \mathrm{F}$ annually, whereas a well 30 feet deep would vary only $1.1^{\circ} \mathrm{C}$ or $2^{\circ} \mathrm{F}$. The variation is nearly zero at about 55 feet. The approximate time required for major seasonal air temperatures to arrive at a given depth is given in figure 7 . 
of ground water, 1960-67

U.S. Geological Survey; MDH, Michigan Department of Health. Analyses prior to 1960 aregiven in Deutsch and numerous industrial analyses are on file at U.S. Geological Survey District Office, Lansing, Mich.]

\begin{tabular}{|c|c|c|c|c|c|c|c|c|c|c|c|}
\hline \multirow{2}{*}{$\begin{array}{l}\text { Bicar- } \\
\text { bonate } \\
\left(\mathrm{HCO}_{3}\right)\end{array}$} & \multirow{2}{*}{$\begin{array}{l}\text { Sul- } \\
\text { fate } \\
\left(\mathrm{SO}_{4}\right)\end{array}$} & \multirow{2}{*}{$\begin{array}{l}\text { Chlo- } \\
\text { ride } \\
\text { (Cl) }\end{array}$} & \multirow{2}{*}{$\begin{array}{c}\text { Fluo- } \\
\text { ride } \\
\text { (F) }\end{array}$} & \multirow{2}{*}{$\begin{array}{c}\mathrm{Ni}- \\
\text { trate } \\
\left(\mathrm{NO}_{3}\right)\end{array}$} & \multirow{2}{*}{$\begin{array}{c}\text { Dis- } \\
\text { solved } \\
\text { solids } \\
\text { (resi- } \\
\text { due at. } \\
180^{\circ} \mathrm{C} \text { ) }\end{array}$} & \multicolumn{2}{|c|}{$\begin{array}{l}\text { Hardness } \\
\text { as } \mathrm{CaCO}_{3}\end{array}$} & \multirow{2}{*}{$\begin{array}{l}\text { Specific } \\
\text { conduct- } \\
\text { ance } \\
\text { (micro- } \\
\text { mhos at } \\
25^{\circ} \mathrm{C} \text { ) }\end{array}$} & \multirow{2}{*}{$\mathrm{pH}$} & \multirow{2}{*}{$\begin{array}{l}\text { Color } \\
\text { (Pt-Co } \\
\text { scale) }\end{array}$} & \multirow{2}{*}{ Analyst } \\
\hline & & & & & & $\begin{array}{l}\text { Calcium, } \\
\text { magne- } \\
\text { sium }\end{array}$ & $\begin{array}{c}\text { Non- } \\
\text { car- } \\
\text { bonate }\end{array}$ & & & & \\
\hline
\end{tabular}

\begin{tabular}{|c|c|c|c|c|c|c|c|c|c|c|c|}
\hline $\begin{array}{l}274 \\
194 \\
384 \\
360 \\
330 \\
314 \\
300 \\
285\end{array}$ & $\begin{array}{c}14 \\
168 \\
43 \\
38 \\
43 \\
58 \\
26\end{array}$ & $\begin{array}{c}6.0 \\
5.0 \\
35 \\
8.0 \\
11 \\
14 \\
26 \\
12\end{array}$ & $\begin{array}{l}0.1 \\
0 \\
0 \\
0 \\
0 \\
0 \\
0\end{array}$ & $\begin{array}{l}0.5 \\
2.5 \\
0 \\
4.0 \\
0 \\
0 \\
0\end{array}$ & $\begin{array}{l}243 \\
206 \\
664 \\
384 \\
364 \\
366 \\
396 \\
306\end{array}$ & $\begin{array}{l}231 \\
192 \\
490 \\
340 \\
320 \\
310 \\
295 \\
230\end{array}$ & $\begin{array}{r}6 \\
33 \\
175 \\
45 \\
49 \\
53 \\
49 \\
0\end{array}$ & $\begin{array}{l}436 \\
368 \\
960 \\
620 \\
600 \\
600 \\
610 \\
480\end{array}$ & $\begin{array}{l}7.3 \\
7.6 \\
7.2 \\
7.4 \\
7.5 \\
7.5 \\
7.8 \\
7.7\end{array}$ & $\begin{array}{r}3 \\
0 \\
0 \\
0 \\
0 \\
0 \\
0\end{array}$ & $\begin{array}{l}\text { USGS } \\
\text { USGS } \\
\text { MDH } \\
\text { MDH } \\
\text { MDH } \\
\text { MDH } \\
\text { MDH } \\
\text { MDH }\end{array}$ \\
\hline $\begin{array}{l}276 \\
336 \\
350 \\
355 \\
375 \\
360 \\
350 \\
302\end{array}$ & $\begin{array}{r}26 \\
47 \\
230 \\
104 \\
188 \\
178 \\
178 \\
30\end{array}$ & $\begin{array}{l}9.0 \\
16 \\
35 \\
31 \\
41 \\
30 \\
28 \\
13\end{array}$ & $\begin{array}{r}.3 \\
.3 \\
.4 \\
.4 \\
.4 \\
.2\end{array}$ & $\begin{array}{c}0 \\
93 \\
3.2 \\
1.0 \\
19 \\
0_{0}^{6}\end{array}$ & $\begin{array}{l}272 \\
491 \\
700 \\
554 \\
706 \\
630 \\
626 \\
316\end{array}$ & $\begin{array}{l}252 \\
371 \\
545 \\
425 \\
515 \\
485 \\
475 \\
280\end{array}$ & $\begin{array}{r}26 \\
95 \\
258 \\
134 \\
207 \\
190 \\
188 \\
32\end{array}$ & $\begin{array}{r}459 \\
774 \\
980 \\
850 \\
1,000 \\
930 \\
910 \\
540\end{array}$ & $\begin{array}{l}7.5 \\
7.3 \\
7.4 \\
7.1 \\
7.0 \\
7.0 \\
7.1 \\
7.7\end{array}$ & $\begin{array}{r}10 \\
0 \\
0 \\
0 \\
0 \\
5\end{array}$ & $\begin{array}{l}\text { USGS } \\
\text { USGS } \\
\text { MDH } \\
\text { MDH } \\
\text { MDH } \\
\text { MDH } \\
\text { MDH } \\
\text { MDH }\end{array}$ \\
\hline $\begin{array}{l}310 \\
303 \\
385 \\
350 \\
340 \\
355 \\
330 \\
445\end{array}$ & $\begin{array}{r}34 \\
12 \\
103 \\
62 \\
55 \\
69 \\
64 \\
172\end{array}$ & $\begin{array}{l}6.0 \\
3.0 \\
45 \\
53 \\
46 \\
58 . \\
60 \\
55\end{array}$ & $\begin{array}{l}0^{2} \\
.1 \\
.1 \\
.1 \\
.3 \\
.4\end{array}$ & $\begin{array}{l}0 \\
0 \\
3.4 \\
0 \\
2.0 \\
.8 \\
.3 \\
.6\end{array}$ & $\begin{array}{l}328 \\
266 \\
558 \\
476 \\
440 \\
498 \\
490 \\
720\end{array}$ & $\begin{array}{l}285 \\
255 \\
430 \\
380 \\
355 \\
370 \\
380 \\
530\end{array}$ & $\begin{array}{r}31 \\
7 \\
114 \\
93 \\
161 \\
79 \\
109 \\
165\end{array}$ & $\begin{array}{r}540 \\
455 \\
920 \\
800 \\
700 \\
820 \\
820 \\
1,080\end{array}$ & $\begin{array}{l}7.7 \\
7.7 \\
7.4 \\
7.7 \\
7.3 \\
7.3 \\
7.4 \\
7.0\end{array}$ & $\begin{array}{l}0 \\
5 \\
0 \\
0 \\
0 \\
0 \\
0 \\
0\end{array}$ & $\begin{array}{l}\text { MDH } \\
\text { MDH } \\
\text { MDH } \\
\text { MDH } \\
\text { MDH } \\
\text { MDH } \\
\text { MDH } \\
\text { MDH }\end{array}$ \\
\hline $\begin{array}{l}350 \\
310 \\
325 \\
325 \\
320 \\
320 \\
355 \\
365\end{array}$ & $\begin{array}{l}94 \\
\mathbf{3 3} \\
\mathbf{5 3} \\
69 \\
\mathbf{5 2} \\
48 \\
86 \\
89\end{array}$ & $\begin{array}{l}38 \\
21 \\
42 \\
26 \\
17 \\
17 \\
58 \\
38\end{array}$ & $\begin{array}{l}0^{2} \\
0 \\
.2 \\
.3 \\
.3 \\
.2 \\
.3\end{array}$ & $\begin{array}{l}10 \\
3.2 \\
1.3 \\
0 \\
0 \\
0 \\
.4 \\
1.8\end{array}$ & $\begin{array}{l}502 \\
354 \\
450 \\
404 \\
366 \\
352 \\
520 \\
500\end{array}$ & $\begin{array}{l}405 \\
295 \\
335 \\
340 \\
315 \\
310 \\
400 \\
385\end{array}$ & $\begin{array}{r}118 \\
41 \\
68 \\
73 \\
53 \\
48 \\
109 \\
86\end{array}$ & $\begin{array}{l}830 \\
580 \\
700 \\
700 \\
640 \\
620 \\
840 \\
800\end{array}$ & $\begin{array}{l}7.2 \\
7.8 \\
7.3 \\
7.4 \\
7.5 \\
7.5 \\
7.7 \\
7.2\end{array}$ & $\begin{array}{l}0 \\
0 \\
0 \\
0 \\
0 \\
0 \\
0 \\
0\end{array}$ & $\begin{array}{l}\text { MDH } \\
\text { MDH } \\
\text { MDH } \\
\text { MDH } \\
\text { MDH } \\
\text { MDH } \\
\text { MDH } \\
\text { MDH }\end{array}$ \\
\hline $\begin{array}{l}300 \\
270 \\
275 \\
260 \\
280 \\
250 \\
240 \\
255\end{array}$ & $\begin{array}{l}8.0 \\
26 \\
24 \\
18 \\
12 \\
40 \\
26 \\
34\end{array}$ & $\begin{array}{l}3.0 \\
4.0 \\
6.0 \\
4.0 \\
2.5 \\
9.0 \\
3.0 \\
14\end{array}$ & $\begin{array}{l}0 \\
.2 \\
.2 \\
.3 \\
.5 \\
.2 \\
.2 \\
.2\end{array}$ & $\begin{array}{l}0 \\
2.3 \\
1.1 \\
.2 \\
0 \\
0 \\
0 \\
0\end{array}$ & $\begin{array}{l}252 \\
274 \\
278 \\
256 \\
252 \\
286 \\
254 \\
308\end{array}$ & $\begin{array}{l}240 \\
250 \\
250 \\
235 \\
225 \\
235 \\
225 \\
250\end{array}$ & $\begin{array}{r}0 \\
29 \\
24 \\
22 \\
0 \\
30 \\
28 \\
41\end{array}$ & $\begin{array}{l}450 \\
430 \\
430 \\
400 \\
418 \\
450 \\
420 \\
460\end{array}$ & $\begin{array}{l}7.6 \\
7.7 \\
7.6 \\
7.6 \\
7.6 \\
8.0 \\
7.7 \\
7.6\end{array}$ & $\begin{array}{l}0 \\
0 \\
0 \\
5 \\
5 \\
0 \\
0 \\
0\end{array}$ & $\begin{array}{l}\text { MDH } \\
\text { MDH } \\
\text { MDH } \\
\text { MDH } \\
\text { USGS } \\
\text { MDH } \\
\text { MDH } \\
\text { MDH }\end{array}$ \\
\hline $\begin{array}{l}285 \\
250 \\
260 \\
278 \\
255 \\
250 \\
265 \\
255\end{array}$ & $\begin{array}{c}35 \\
64 \\
54 \\
21 \\
8.0 \\
32 \\
24 \\
15\end{array}$ & $\begin{array}{l}6.0 \\
10 \\
11 \\
3.0 \\
3.0 \\
2.0 \\
3.0 \\
3.0\end{array}$ & $\begin{array}{l}.2 \\
.2 \\
.2 \\
0 \\
0 \\
0 \\
0 \\
0\end{array}$ & $\begin{array}{l}0 \\
0 \\
0 \\
0 \\
0 \\
0 \\
0^{.5}\end{array}$ & $\begin{array}{l}310 \\
336 \\
322 \\
266 \\
244 \\
266 \\
260 \\
254\end{array}$ & $\begin{array}{l}280 \\
265 \\
270 \\
240 \\
215 \\
235 \\
245 \\
225\end{array}$ & $\begin{array}{r}46 \\
60 \\
57 \\
12 \\
6 \\
30 \\
28 \\
16\end{array}$ & $\begin{array}{l}510 \\
490 \\
500 \\
440 \\
400 \\
440 \\
470 \\
400\end{array}$ & $\begin{array}{l}7.6 \\
7.6 \\
7.6 \\
7.8 \\
7.7 \\
7.6 \\
7.5 \\
7.7\end{array}$ & $\begin{array}{l}0 \\
0 \\
0 \\
0 \\
5 \\
0 \\
0 \\
5\end{array}$ & $\begin{array}{l}\text { MDH } \\
\text { MDH } \\
\text { MDH } \\
\text { MDH } \\
\text { MDH } \\
\text { MDH } \\
\text { MDH } \\
\text { MDH }\end{array}$ \\
\hline $\begin{array}{l}220 \\
272 \\
294 \\
208 \\
222 \\
215 \\
208 \\
220 \\
280\end{array}$ & $\begin{array}{l}0 \\
8.0 \\
12 \\
14 \\
2.0 \\
3.0 \\
1.6 \\
31 \\
27\end{array}$ & $\begin{array}{l}4.0 \\
5.0 \\
5.0 \\
4.0 \\
2.0 \\
2.0 \\
1.5 \\
4.0 \\
2.0\end{array}$ & 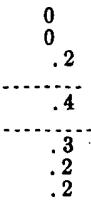 & $\begin{array}{l}0 \\
0 \\
0 \\
.1 \\
.3 \\
01.0 \\
0 \\
0\end{array}$ & $\begin{array}{l}200 \\
244 \\
256 \\
148 \\
208 \\
-196 \\
218 \\
298\end{array}$ & $\begin{array}{l}165 \\
225 \\
235 \\
177 \\
170 \\
171 \\
162 \\
206 \\
251\end{array}$ & $\begin{array}{r}0 \\
2 \\
0 \\
0 \\
0 \\
0 \\
0 \\
26 \\
21\end{array}$ & $\begin{array}{l}350 \\
450 \\
460 \\
336 \\
331 \\
308 \\
390 \\
463\end{array}$ & $\begin{array}{l}7.6 \\
7.5 \\
7.6 \\
7.3 \\
7.6 \\
7.6 \\
7.8 \\
7.7\end{array}$ & $\begin{array}{c}0 \\
5 \\
5 \\
5 \\
5 \\
5 \\
5 \\
5\end{array}$ & $\begin{array}{l}\text { MDH } \\
\text { MDH } \\
\text { MDH } \\
\text { USGS } \\
\text { USGS } \\
\text { USGS } \\
\text { USGS } \\
\text { USGS } \\
\text { USGS }\end{array}$ \\
\hline
\end{tabular}

Although the range in ground-water temperatures for wells less than 55 feet deep varies with depth of well, the average temperature for each well is very close to $10.1^{\circ} \mathrm{C}\left(50.4^{\circ} \mathrm{F}\right)$. This value corresponds closely to the average annual air temperature of about $9.8^{\circ} \mathrm{C}$ 
$\left(49.6^{\circ} \mathrm{F}\right)$. At depths greater than 55 feet, temperature increases about $0.9^{\circ} \mathrm{C}\left(1.6^{\circ} \mathrm{F}\right)$ for each 100 feet of depth as the result of heat flow from the earth's interior (Graf and others, 1965).

The approximate temperature of water in Kalamazoo County at depths greater than 55 feet can be calculated by the following formulas:

$$
\begin{aligned}
& \left.T \text { in }{ }^{\circ} \mathrm{F}=50+0.016 \text { (depth of well in feet }-55\right), \text { and } \\
& \left.T \text { in }{ }^{\circ} \mathrm{C}=10+0.009 \text { (depth of well in feet }-55\right) \text {. }
\end{aligned}
$$

For example, to determine the temperature of water from a well which obtains water from a zone about 100 feet deep: $T\left({ }^{\circ} \mathrm{F}\right)=$ $50+0.016 \times 45=50.7$. Results from the above formulas will be in error if the well is affected by induced surface recharge or high ground-water velocities. However, the formulas are applicable to most domestic and smaller industrial wells.

\section{WATER QUALITY IN RELATION TO USE}

The various uses of water have differing quality requirements. Water that is of excellent quality for one use may be entirely unsatisfactory for another. Probably the most restrictive requirements are those for drinking water. Industrial requirements vary with the industry, but usually are not as restrictive as requirements for public supplies, although some industrial processes demand water of extremely high quality. Agricultural requirements also are normally less restrictive than those of water used for human consumption.

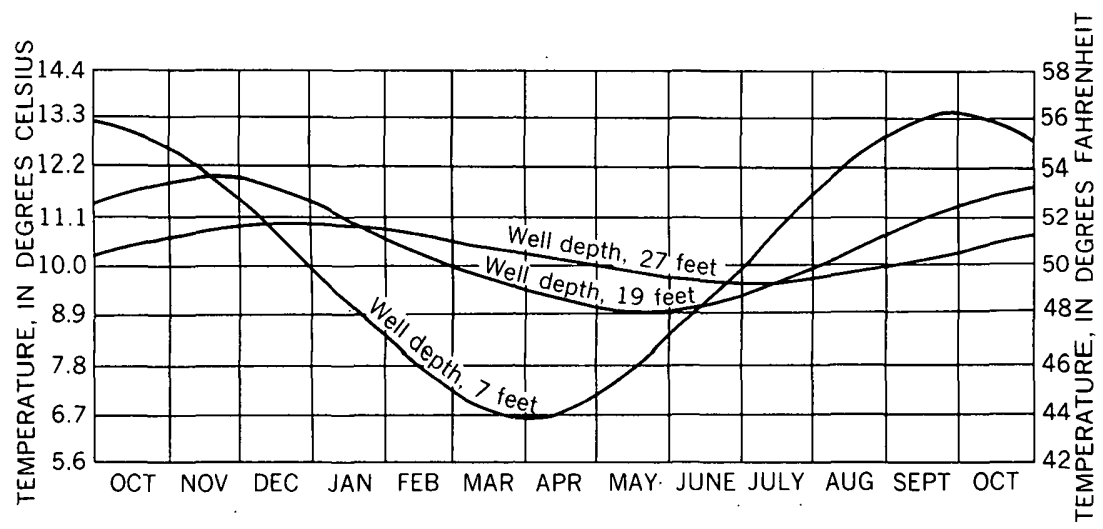

Fraure 5.-Thermographs of selected wells in 1965 and 1966.

PUBLIC AND DOMESTIC SUPPLIES

All water presently used for public and individual domestic supplies in Kalamazoo County is withdrawn from wells in unconsolidated deposits and bedrock that composes the upper several hundred 


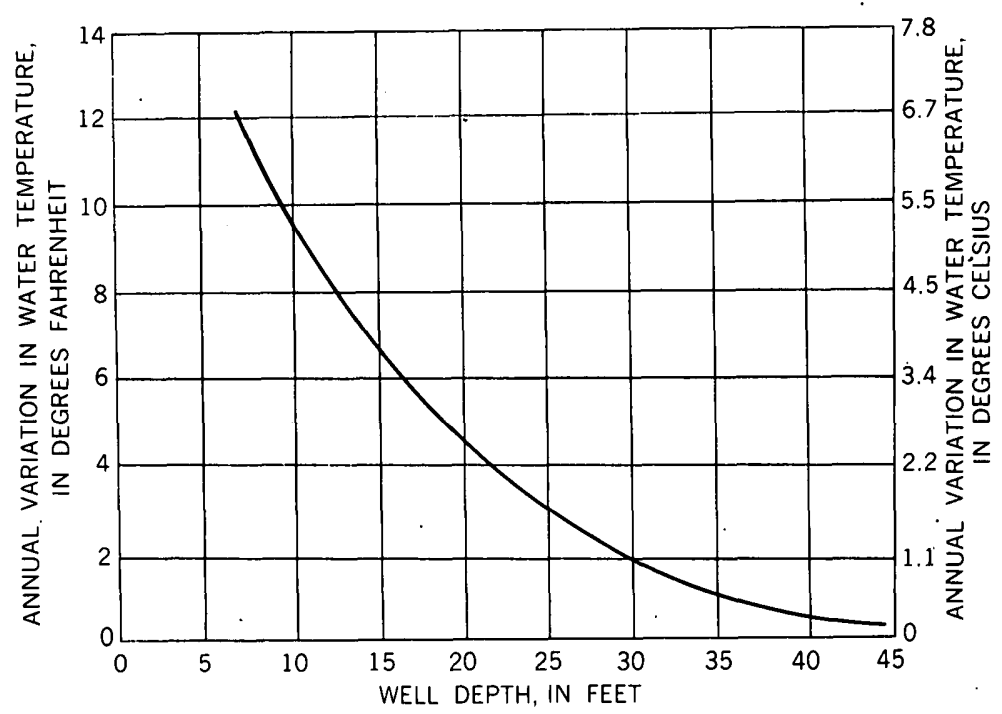

FIaURE 6.-Relationship between annual variations in ground-water temperature and depth of well.

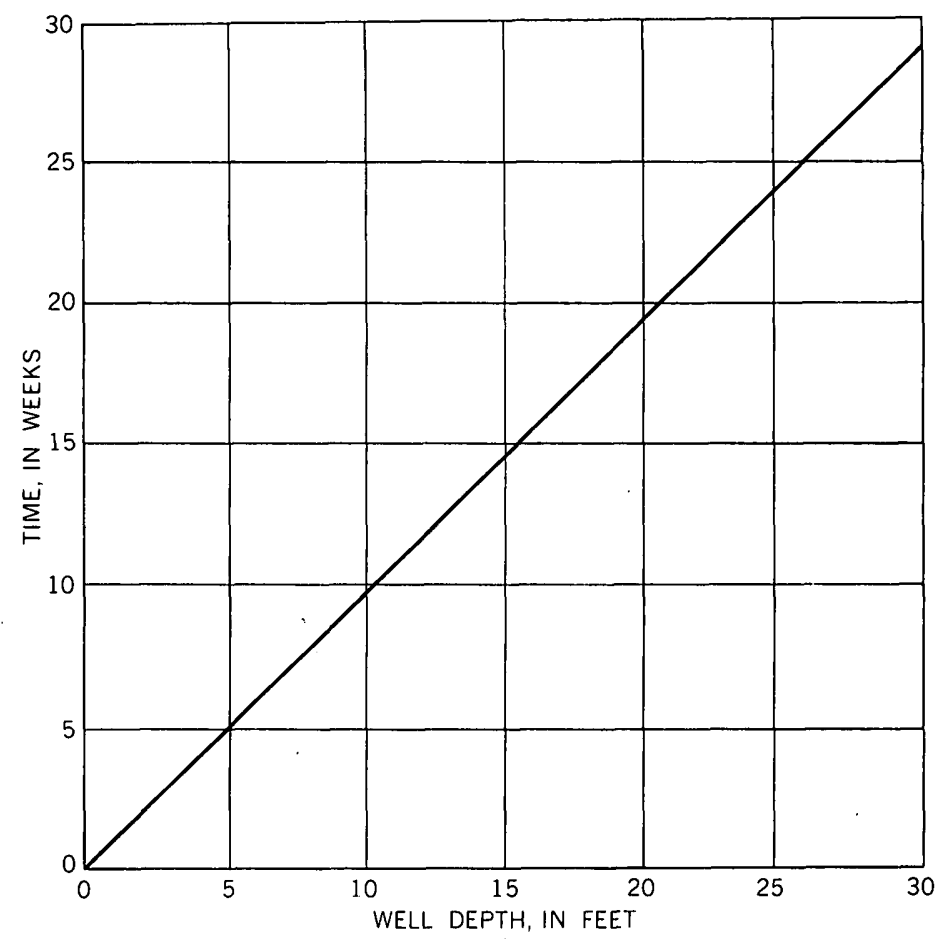

Froure 7.-Relationship between depth of well and arrival time of surface helat. 
feet of earth. Compared to river or lake water, these ground-water sources result in both quality advantages and disadvantages to the consumer. A distinct advantage in the use of ground water is the removal of most pathogenic bacteria by the filtering action of the aquifer. Additionally, the temperature and composition of ground water tend to remain relatively uniform throughout the year thereby allowing for more uniform treatment procedures. A disadvantage in the use of ground water is the greater amounts of dissolved solids, particularly calcium and magnesium which cause hardness, and the high iron content.

The suitability of ground water for municipal use in Kalamazoo County is determined chiefly by the hardness and iron content of the water. Of the six major ions present in water, the U.S. Public Health Service (1962) recommends maximums on two of them, chloride at $250 \mathrm{mg} / \mathrm{l}$ (milligrams per liter) and sulfate at $250 \mathrm{mg} / \mathrm{l}$. Hardness is classified according to Swenson and Baldwin (1965) as follows, but no recommended maximums are stated.

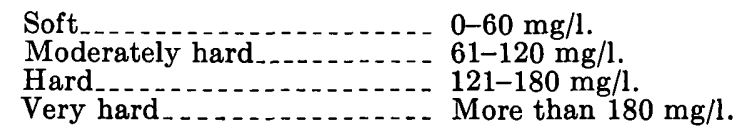

Hardness in water is a lesser problem with modern soaps. Most people, for example, consider water of $180 \mathrm{mg} / \mathrm{l}$ hardness satisfactory for household use.

Analyses of river water within the county indicate that, with the exception of parts of Kalamazoo River, Portage Creek, and Allen Creek, the streams in the county would provide water of good quality for municipal supplies-relatively low in concentration of iron and dissolved solids and in hardness. Use of river water, however, would generally require reservoir construction and more extensive biologic treatment, filtering, aerating, and color treatment. Lakes could also serve as sources of excellent quality water for domestic and municipal supplies with biological treatment; however, most such use would interfere with the recreation and esthetic uses of most lakes in the area.

The ground water is generally of good quality, although the water is hard and generally has objectionably high iron content. The major planning emphasis should be directed toward preventing contamination of the aquifers either by direct disposal, including sanitary fill, or by infiltration of undesirable water from surface sources. Deterioration of the quality could also occur if the water levels in deep wells in the unconsolidated aquifers become significantly lower than the water levels in wells penetrating bedrock. 


\section{INDUSTRIAL SUPPLIES}

The industrial requirements for water quality and temperature are dependent upon use and product; however, most of the water withdrawn by industry in Kalamazoo County is used for cooling purposes. The relatively uniform temperature of ground water, approximately $10^{\circ} \mathrm{C}\left(50^{\circ} \mathrm{F}\right)$, and predictable supply allow the manager flexibility in production scheduling. The major disadvantage of ground water in Kalamazoo County for cooling is hardness and its saturation with calcium and magnesium bicarbonate. When the water is heated, precipitation of calcium or magnesium carbonate occurs, thereby creating a "scale" and consequent reduction of the heatexchange rate. Iron precipitation can also be a problem if the system is open to oxygen at any point, because most well water in the county contains objectionable quantities of iron, which forms a precipitate when water comes in contact with oxygen.

The use of river water generally reduces the problem of iron precipitation and, to a lesser extent, that of hardness; however, about 70 percent of the annual flow of the Kalamazoo River is derived directly from ground water and is very hard (table 11). Average monthly river temperatures under natural conditions are nearly equal to average monthly air temperatures except during December, January, and February, when air temperatures are below the freezing temperature of water. Air temperature records collected at Kalamazoo State Hospital exhibit the following monthly averages in degrees Fahrenheit:

Jan. Feb. Mar. Apr. May June July Aug. Sept. Oct. Nov. Dec.

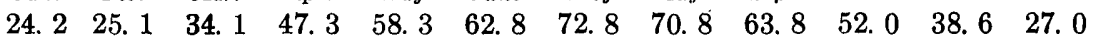

Daily river temperatures vary considerably from the monthly average as shown by data collected on July 27 and 28, 1964 .

\begin{tabular}{|c|c|c|c|c|}
\hline \multirow{2}{*}{ Station } & \multirow{2}{*}{ Stream and locality } & \multicolumn{2}{|c|}{ Temperature } & \multirow{2}{*}{$\begin{array}{l}\text { Discharge } \\
\text { in efs }\end{array}$} \\
\hline & & ${ }^{\circ} \mathrm{C}$ & ${ }^{\circ} \mathrm{F}$ & \\
\hline $\begin{array}{l}1060.00 \\
1064.00 \\
971.70 \\
972.00 \\
1057.00 \\
1063.00\end{array}$ & $\begin{array}{l}\text { Kalamazoo River at Comstock } \\
\text { West Fork Portage River at Kalamazoo. } \\
\text { Portage River near Vicksburg } \\
\text { Gourdneck Creek near Schoolcraft } \\
\text { Augusta Creek near Augusta } \\
\text { Portage Creek near Kalamazoo }\end{array}$ & $\begin{array}{l}32.8 \\
-21.7 \\
-29.4 \\
-28.3 \\
-21.2\end{array}$ & $\begin{array}{l}91 \\
89 \\
85 \\
83 \\
76 \\
71\end{array}$ & $\begin{array}{r}* 300 \\
1.62 \\
\text { 7. } 04 \\
* 1.40 \\
\text { 14. } 0 \\
\text { 35. } 0\end{array}$ \\
\hline
\end{tabular}

*Daily mean discharge.

Diurnal fluctuations are not considered in these readings, and therefore, the values do not represent daily averages. The data, how- 
ever, show the temperatures of the various rivers under approximately the same climatic conditions. The heating effect of lakes appears important in the first four streams; however, the Kalamazoo River at Comstock is also affected by heat produced at Morrow Pond generating station. In general the percentage of ground water locally contributed to the total flow has a significant effect on the temperature. Two examples of this are West Fork Portage Creek, which loses water over most of its lower reach and has a high summer temperature, and Portage Creek near Kalamazoo, which has the largest contribution of ground water per unit area in the county and has a relatively low summer temperature.

Use of river water for cooling during winter and of ground water for cooling during summer can be an effective management technique. It not only results in better cooling efficiency, but also reduces the thermal load of the stream in the summer, when dissolved oxygen is low. Where the river is hydrologically connected to the aquifer, the aquifer can act as a heat sink for warm summer water and a heat source for cool winter water with an overall temperature-stabilizing effect. This stabilizing effect is dependent upon the size of the aquifer, local transmissibility, and amount of water induced into the aquifer. In general, the farther from the recharging source either vertically or horizontally, the more stable the resulting temperature will be. Calculations of the percentage of river water infiltrated can be made using a method developed by Theis (1941). The effectiveness of an installation can be evaluated by comparing observed water temperatures with those of unpumped aquifers (figs. 6 and 7). However, certain problems can arise with an infiltration system. Sealing of the river bottom by silt and pollution products reduces the rate of induced recharge. Much of the water infiltrated from the Kalamazoo River and the lower reach of Portage Creek near Kalamazoo contains significant quantities of calcium sulfate. This could adversely affect the aquifer by causing precipitation of calcium carbonate and reducing the permeability of the aquifer materials.

The use of river water for cooling can reduce the cooling efficiency to downstream users. The river will cool to normal temperature in time through heat exchange with the atmosphere. The time needed is relatively long compared to river velocity; consequently, the water generally is not completely cooled until it flows out of the county.

The Michigan Water Resources Commission (1968) is presently establishing thermal loading criteria for streams in Michigan. Industries using water for cooling will be required to conform to these criteria. These criteria may have the effect of increasing the industrial use of ground water for cooling purposes. 


\section{AGRIGULTURAL SUPPLIES}

The chemical quality of ground water and most surface water in Kalamazoo County is acceptable for stock and poultry use. The only exceptions might be surface water in the reach of the Kalamazno River from Comstock to Plainwell, lower Portage Creek near Kalamazoo, and Allen Creek, all of which have a large amount of suspended material.

Chemical quality of water is important to irrigation. The quality not only affects plant growth directly, but also can affect the tilth of the soil. In general, water available in humid areas is quite satisfactory for irrigation. Water too highly mineralized for public and industrial supplies may be suitable for irrigation of many crops. However, there are definite quality requirements for irrigation water. The U.S. Salinity Laboratory staff (1954) classifies the suitability of irrigation water based on four hazards: sodium, salinity (total dissolved solids), bicarbonate, and boron. Most emphasis is given to the sodium and salinity hazards, with phytotoxic constituents, including boron, considered as additional limiting factors. The classification of these hazards is general and should be considered in relation to crop management, drainage, rainfall, evapotranspiration, and plant sensitivity. The chemical quality of both ground and surface water in Kalamazoo County is good for irrigation based on criteria of sodium, salinity, and bicarbonate hazards. Any proposed irrigation source should be analyzed for boron.

\section{AVAILABILITY OF WATER}

The availability of water is controlled by the characteristics of the geohydrologic system. Water must also withstand certain tests of quality. In Kalamazoo County water is available in large quantities and generally is of good chemical quality. Lakes and streams are sources of water for immediate development. Gull Lake and Kalamazoo River both have abundant supplies of water readily available, and the quality is generally adequate for most uses. Abundant supplies of relatively good quality water that meet most of the recommended limits (U.S. Public Health Service, 1962) can also be obtained from ground-water reservoirs.

\section{STREAMS}

The availability of water in streams for withdrawal use is related to the volume of streamflow. The primary need for water is for the dilution of waste materials, water supply, irrigation, and recreational and esthetic uses of the streams. In order to plan and manage the total uses of the streams, it is essential to know the flow characteristics as well as the water quality. 
Basic data on streamflow were collected at more than 60 sites in Kalamazoo County during the period 1964-67. The stations listed in figure 8 show streamflow data at gaging stations and low-flow partialrecord sites. In addition, data were collected at several miscellaneous sites not listed in figure 8 . These data include a minimum of three measurements of discharge at times when the streamflow was unaffected by surface runoff.

The gaging station, Kalamazoo River at Comstock, has the longest streamflow record within the county. The records at other sites listed in figure 8 contained either a continuous record of daily discharge over a relatively short period or a fragmentary record consisting of several base-flow discharge measurements made periodically over a period of several years. Records collected for only short periods generally do not include the variations of flow typical of a long period because they may have been collected during a period of unusually wet or dry years. Because most of the stations in the county have short periods of record, the characteristics of streamflow for the stations were determined by statistical correlation with long-term stations within and adjacent to the county. In order to include periods of both wet and dry years, data were extended to the base period 1931-66. In the sections that follow, definition of the character-

STATION

WATER YEAR ENDING SEPTEMBER 30

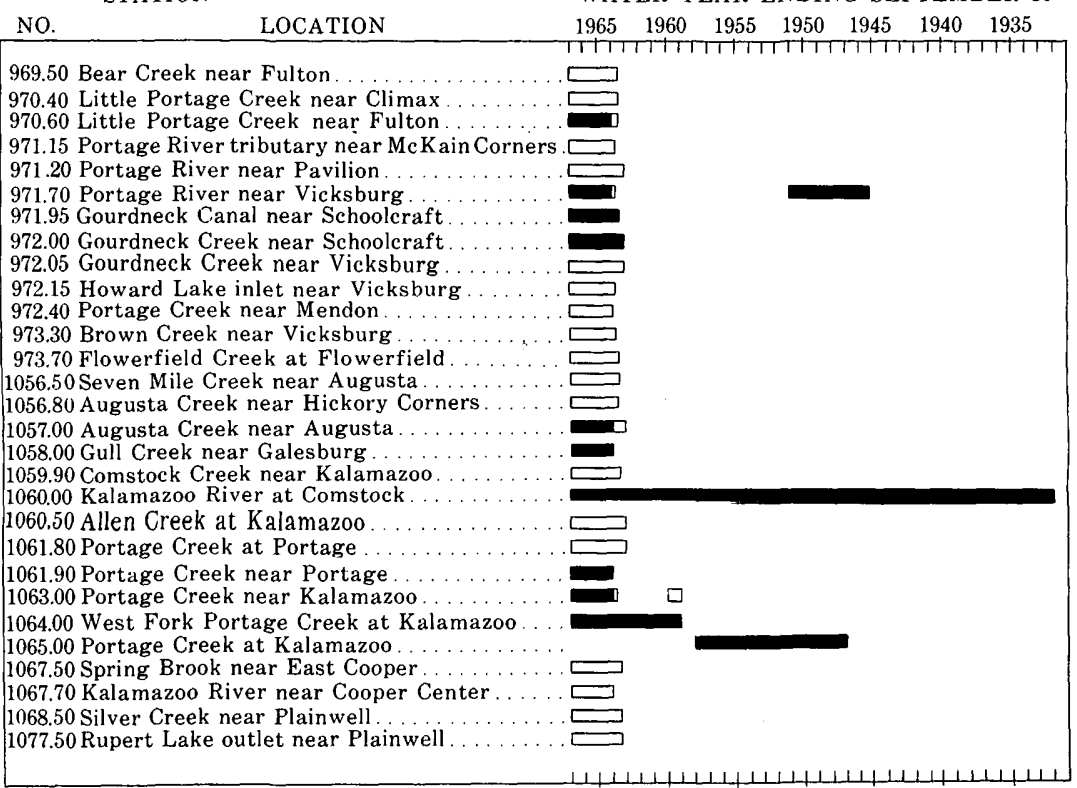

FrguRe 8.-Records of streamflow data. Open bar, occasional discharge measurements ; solid bar, streamflow measurements. 
istics of streamflow is presented in convenient form for use by water managers and planners. The methods of analysis follow recognized procedures and will be described only where necessary to make the results clear to the reader.

\section{FLOW DURATION}

A flow-duration curve is a cumulative frequency curve that shows the percentage of time, during the period studied, that any given rate of flow was equaled or exceeded. It provides a useful device for analyzing the availability and variability of streamflow without regard to the sequence of flow. The distribution of streamflow with respect to time is dependent upon topography, climate, type of land cover, soil condition, geology, and other factors. The shape of the flow-duration curve is determined by these characteristics.

The slope of the flow-duration curve is a good indication of the amount of storage within a basin. Most of the water stored in Kalamazoo County is in ground-water reservoirs, but lakes and marshes also store water. The steeper the duration curve, the greater the variability in streamflow; that is, the stream has a wide range of discharge and little natural storage. When a flow-duration curve has a flat slope, a high sustained flow per unit area is indicated. This high sustained flow results from water going into storage during wet periods to be gradually released through ground-water seepage or outflow from lakes and marshes to streams. Figure 9 shows the duration of daily flows in cubic feet per second per square mile for five streams in the county for the period 1965-66. Differences in the curves are in their general slopes. Little Portage Creek and Portage River near Vicksburg have the steeper slopes. This is because these streams drain areas where the transmissibility of the glacial drift is generally low (pl. 2). In contrast, the flow-duration curves for Kalamazoo River, Portage Creek near Portage, and Augusta Creek have flatter slopes and higher sustained flow per unit area during dry periods because they drain areas where the transmissibility is generally high.

Flow-duration curves, adjusted to the base period 1931-66, were prepared for several long-term stations in and adjacent to Kalamazoo County. The extensions were made by the use of correlation procedures. Estimates of the 50- and 90-percent duration values for other sites listed in table 9 were then determined by correlating data at these sites with those of the long-term stations. The 50-percent point on the duration curve represents the median discharge of the stream. The 90-prcent point is often used as an index of low flow because the point is sufficiently far from the minimum to be fairly stable and yet low enough to be significant. 


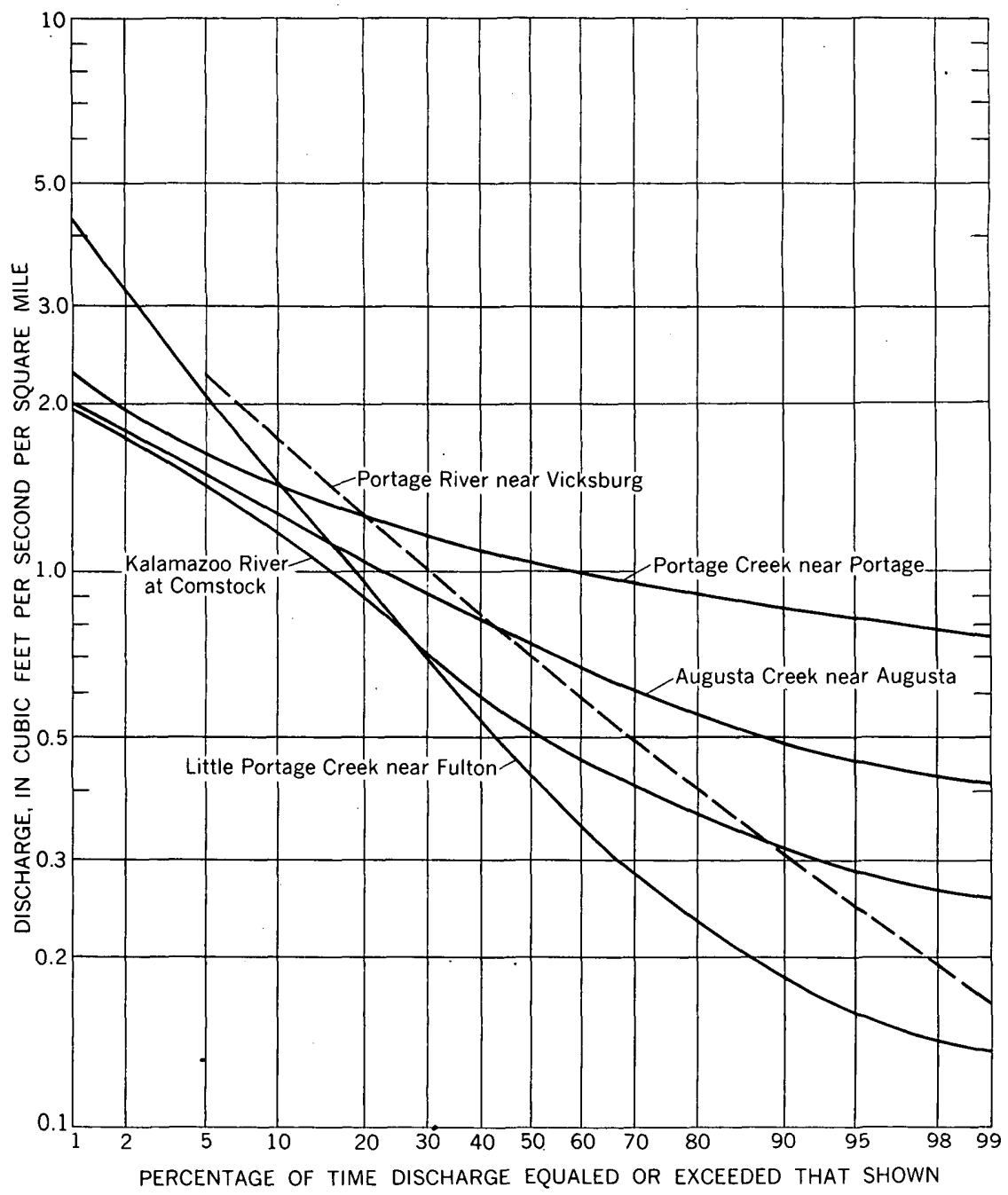

FIGURE 9.-Flow-duration curves of daily discharge for selected gaging stations in 1965 and 1966.

Many of the values of the 90 -percent duration point are about the same as the 7-day low flows that occur on an average at 2-year recurrence intervals. The 7-day low flows are the low-flow index used in this report and will be discussed in more detail in the following sections.

\section{LOW-FLOW FREQUENCY}

The flow of streams in the county is lowest in the summer and early fall, which is the time of greatest demand for water. Increased uses of water for cooling purposes, irrigation, lawn sprinkling, and other uses 


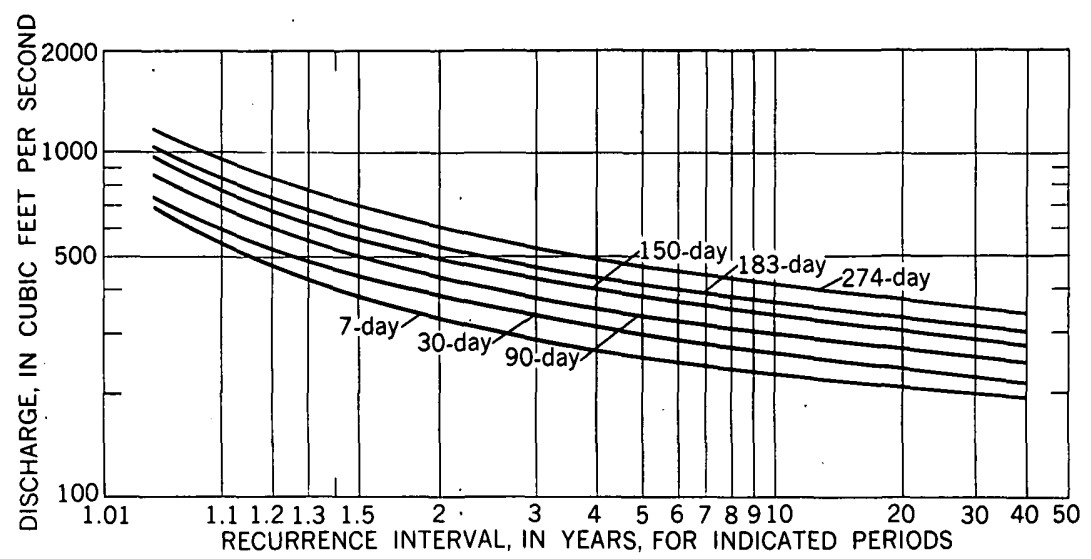

FIGURE 9.-Flow-duration curves of daily discharge for selected gaging stations adjusted to period 1931-65.

occur during these seasons. Unless storage is provided, the amount of water needed during these periods often exceeds the available supply. The frequency with which low flows occur is, therefore, an important factor when determining the potential of a stream to furnish needed water. As previously stated, flow-duration curves do not indicate the sequence of flows. The curves show the percentage of time that a specified flow was equaled or exceeded during a given period, but they do not indicate whether that flow occurred consecutively on many days in a single year or whether it occurred on a few days in each year. Low-flow frequency curves relate the lowest average discharge for various periods to the interval of recurrence of this discharge. Figure 10 contains a family of curves for Kalamazoo River at Comstock showing the magnitude and frequency of low flows. In low-flow frequency studies, data by climatic years (April 1 to March 31) are analyzed in order to contain the low-water season within an annual period. The curves for the Comstock station show that, on an average of about once in 20 years, the average flow for 7-day and 90-day periods are expected to be as low, or lower than, 210 and $270 \mathrm{cfs}$, respectively.

The recurrence interval of a specified low flow is the average number of years between occurrences of an equal or smaller flow. Mathematically, the recurrence interval is the reciprocal of the probability of occurrence. For example, a flow having a 50-percent chance of occurring in any given year has a recurrence interval of $1 / 0.50$, or 2 years; similarly, a 5-percent chance of occurrence is equivalent to a recurrence interval of $1 / 0.05$, or 20 years. A given recurrence interval must not be interpreted as the exact time interval between 
TABLE 9.-Streamflow characteristics at

[Data adjusted to period 1931-65. Discharge and low. flow in cublc

\begin{tabular}{|c|c|c|}
\hline Station & Gaging site & $\begin{array}{c}\text { Drainage } \\
\text { area } \\
\text { (sq mi) }\end{array}$ \\
\hline $0969.50 \ldots$ & Bear Creek near Fulton............... & 10.3 \\
\hline $0970.40 \ldots \ldots$ & Little Portage Creek near Climax & 10.1 \\
\hline $0970.60 \ldots$. & Little Portage Creek near Fulton........ & 27.0 \\
\hline $0971.15 \ldots$ & Portage River tributary near McKain Corners.. & 8.47 \\
\hline $0971.20 \ldots$ & Portage River near Pavilion... & 32.8 \\
\hline $0971.30 \ldots$ & Dorrance Creek at McKain Corners..... & 13.0 \\
\hline $0971.70 \ldots$. & Portage River near Vicksburg. & 68.2 \\
\hline $0972.00 \ldots$. & Gourdneck Creek near Schoolcraft $1 \ldots . . .$. & 7. 29 \\
\hline $0972.05 \ldots \ldots . .$. & Gourdneck Creek near Vicksburg ${ }^{2} \ldots$ & 13.1 \\
\hline $0972.40 \ldots \ldots$. & Portage Creek near Mendon & 57.7 \\
\hline $0973.30 \ldots . .$. & Brown Creek near Vicksburg ............... & 13.1 \\
\hline $0973.70 \ldots . . .$. & Flowerfield Creek at Flowerfield.......... & 42.6 \\
\hline $1056.60 \ldots . . . .$. & Seven Mile Creek near Augusta............ & 14.4 \\
\hline $1056.80 \ldots \ldots$ & Augusta Creek near Hickory Corners...- & 19.6 \\
\hline $1057.00 \ldots \ldots$. & Augusta Creek near Augusta................... & 38.9 \\
\hline $1058.00 \ldots$ & Gull Creek near Galesburg...................... & 38.1 \\
\hline $1059.90 \ldots \ldots . . .$. & Comstock Creek near Kalamazoo....... & 18.3 \\
\hline $1060.00 \ldots \ldots . . .$. & Kalamazoo River at Comstock......... & ${ }^{3} 1010$ \\
\hline $1060.50 \ldots$ & Allen Creek at Kalamazoo................. & 15.0 \\
\hline $1061.80 \ldots . . . .$. & Portage Creek at Portage......... & 16.5 \\
\hline $1061.90 \ldots . . . .$. & Portage Creek near Portage........... & 18.6 \\
\hline $1063.00 \ldots$ & 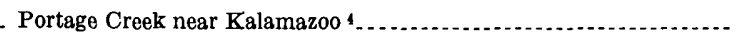 & 22.4 \\
\hline $1064.00 \ldots \ldots . . . .$. & West Fork Portage Creek at Kalamazoo...... & 18.7 \\
\hline $1067.50 \ldots \ldots \ldots . . . .$. & Spring Brook near East Cooper............. & 31.1 \\
\hline $1068.50 \ldots . . . . .$. & 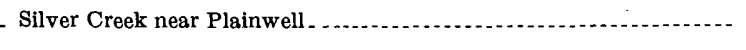 & 20.5 \\
\hline $1077.50 \ldots \ldots . . .$. & Rupert Lake outlet near Plainwell......... & 5. 27 \\
\hline
\end{tabular}

\footnotetext{
1 Discharge adjusted for diversion.

2 Discharge not adjusted for diversion.
}

similar events, but is the average time between occurrences. Thus a 7 -day average flow of $210 \mathrm{cfs}$ or less may occur in 2 successive years, but chances are that only five such events will occur in a 100-year period. Data on the magnitude and frequency of the average 7-day and 30-day low flows at gaging and partial-record sites are given in table 9.

During the period 1964-67, several base-flow measurements were obtained at miscellaneous sites throughout the county. These measured flows were correlated with nearby gaging-station records. Where the degree of correlation was satisfactory, estimates of 7-day and 30-day 
gaging and partial-record stations

feet per second and in cubic feet per second per square mile (italicized)]

\begin{tabular}{|c|c|c|c|c|c|c|c|c|}
\hline \multirow{2}{*}{$\begin{array}{c}\text { Average } \\
\text { discharge }\end{array}$} & \multicolumn{2}{|c|}{$\begin{array}{l}\text { Discharge equaled or } \\
\text { exceeded for percentage } \\
\text { of time shown }\end{array}$} & \multicolumn{3}{|c|}{$\begin{array}{l}\text { Average } 7 \text {-day low flow for the } \\
\text { recurrence lnterval shown }\end{array}$} & \multicolumn{3}{|c|}{$\begin{array}{l}\text { Average } 30 \text {-day low flow for the } \\
\text { recurrence Interval shown }\end{array}$} \\
\hline & or tor & Hown & 2-yr & $10-\mathrm{yr}$ & $20-\mathrm{yr}$ & 2-yr & $10-\mathrm{yr}$ & $20-y r$ \\
\hline 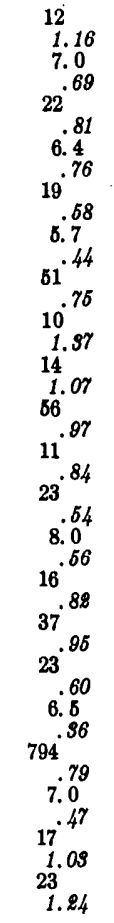 & 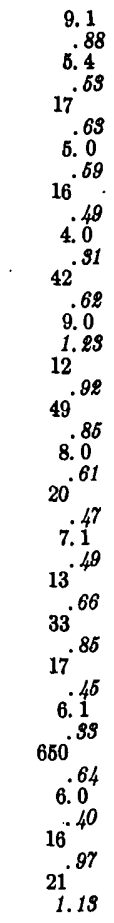 & $\begin{array}{c}3.0 \\
. .28 \\
1.0 \\
.10 \\
6.8 \\
.21 \\
2.7 \\
.92 \\
8.9 \\
.27 \\
1.3 \\
17^{.10} \\
.26 \\
6.5 \\
. .89 \\
6.7 \\
27 \\
.51 \\
2.47 \\
11.20 \\
.26 \\
5.0 \\
.95 \\
7.2 \\
.97 \\
21 \\
.54 \\
6.1 \\
.16 \\
3.7 \\
360 \\
.20 \\
.96 \\
3.3 \\
13.28 \\
17.78 \\
.91\end{array}$ & $\begin{array}{c}2.0 \\
.18 \\
.8 \\
.08 \\
5.0 \\
.18 \\
2.5 \\
.90 \\
8.2 \\
.25 \\
1.1 \\
17.08 \\
17 \\
6.35 \\
.86 \\
5.6 \\
26.49 \\
26 \\
.45 \\
2.3 \\
.18 \\
9.2 \\
.28 \\
4.7 \\
.98 \\
6.3 \\
.92 \\
20 \\
.51 \\
5.1 \\
.19 \\
3.3 \\
.18 \\
330 \\
.93 \\
3.1 \\
.21 \\
12.79 \\
17.79 \\
.91\end{array}$ & $\begin{array}{c}0.9 \\
.09 \\
.16 \\
.016 \\
2.7 \\
.10 \\
1.7 \\
.20 \\
5.6 \\
.17 \\
.5 \\
.04 \\
11.16 \\
.16 \\
4.6 \\
.68 \\
3.4 \\
.26 \\
18 \\
.81 \\
1.2 \\
.09 \\
3.1 \\
.07 \\
3.5 \\
.84 \\
4.1 \\
.21 \\
15 \\
.99 \\
2.6 \\
.07 \\
1.8 \\
.10 \\
230 \\
.29 \\
1.8 \\
.12 \\
9.5 \\
.68 \\
15 \\
.81\end{array}$ & $\begin{array}{c}0.7 \\
.07 \\
.08 \\
.008 \\
2.2 \\
.08 \\
1.5 \\
4.18 \\
4.9 \\
.15 \\
.4 \\
.09 \\
8.1 \\
.18 \\
4.2 \\
.58 \\
3.0 \\
1.28 \\
16 \\
.28 \\
1.0 \\
.08 \\
2.1 \\
.05 \\
3.2 \\
.22 \\
3.5 \\
.18 \\
14 \\
.96 \\
2.2 \\
.06 \\
1.8 \\
210 \\
.08 \\
.21 \\
1.6 \\
.11 \\
8.9 \\
.54 \\
14.04 \\
.76\end{array}$ & $\begin{array}{c}2.5 \\
.84 \\
1.1 \\
.11 \\
6.8 \\
.21 \\
2.8 \\
.89 \\
9.0 \\
.87 \\
1.3 \\
18.10 \\
.26 \\
6.8 \\
.98 \\
6.3 \\
29.48 \\
.50 \\
2.6 \\
11.20 \\
.26 \\
6.2 \\
.96 \\
7.1 \\
2.96 \\
.67 \\
6.4 \\
.17 \\
3.8 \\
380.81 \\
.98 \\
3.3 \\
13.28 \\
18 \\
.78 \\
.87\end{array}$ & $\begin{array}{c}1.1 \\
.11 \\
.2 \\
.08 \\
2.9 \\
.11 \\
2.0 \\
.24 \\
6.2 \\
.18 \\
.6 \\
.05 \\
11.16 \\
.16 \\
5.0 \\
.69 \\
4.0 \\
.91 \\
20 \\
.95 \\
1.4 \\
.11 \\
4.0 \\
.09 \\
3.8 \\
.26 \\
4.6 \\
16.89 \\
3.41 \\
3.1 \\
.08 \\
2.2 \\
.18 \\
270 \\
.87 \\
2.2 \\
10.15 \\
15 \\
.61 \\
.81\end{array}$ & 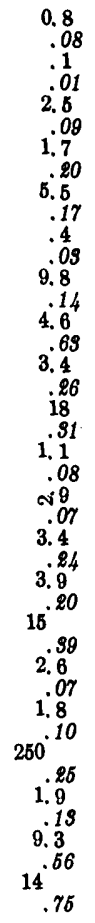 \\
\hline $\begin{array}{c}10^{.58} \\
17^{.65} \\
12.59 \\
9.5 \\
1.80\end{array}$ & $\begin{array}{c}8.8 \\
15^{.47} \\
10^{.48} \\
7.49 \\
1.50\end{array}$ & $\begin{array}{c}4.8 \\
11.26 \\
.95 \\
7.2 \\
.95 \\
5.6 \\
1.06\end{array}$ & $\begin{array}{c}4.8 \\
10^{.26} \\
.92 \\
6.8 \\
.99 \\
5.3 \\
1.01\end{array}$ & $\begin{array}{l}1.9 \\
.10 \\
9.4 \\
.30 \\
5.8 \\
.88 \\
4.3 \\
.88\end{array}$ & $\begin{array}{l}1.4 \\
.07 \\
9.2 \\
.80 \\
5.6 \\
.87 \\
4.1 \\
.78\end{array}$ & $\begin{array}{c}5.8 \\
11.91 \\
.95 \\
7.2 \\
.95 \\
6.7 \\
1.08\end{array}$ & $\begin{array}{l}2.8 \\
.15 \\
9.8 \\
.82 \\
6.0 \\
.29 \\
4.6 \\
.87\end{array}$ & $\begin{array}{l}2.2 \\
.18 \\
9.5 \\
.81 \\
5.8 \\
.28 \\
4.3 \\
.82\end{array}$ \\
\hline
\end{tabular}

3 Drainage area approximate.

- Discharge affected by pumpage of ground water into stream by industry.

flows for 2-year and 10-year recurrence intervals were made. The results are shown in table 10 .

In order to show the availability of streamflow, the flow characteristics for gaging and partial-record stations have been shown on plate 5. Included are values of average discharge, median discharge, and 7-day low flow at a 2-year recurrence interval. Location of miscellaneous sites are also shown.

Each site shown on plate 5 is identified by a station number. These numbers conform to the numbering system used since 1958 for the 
TABLE 10.-Estimates of 7- and 30-day low flows for 2- and

[Data adjusted

Station

Location of site

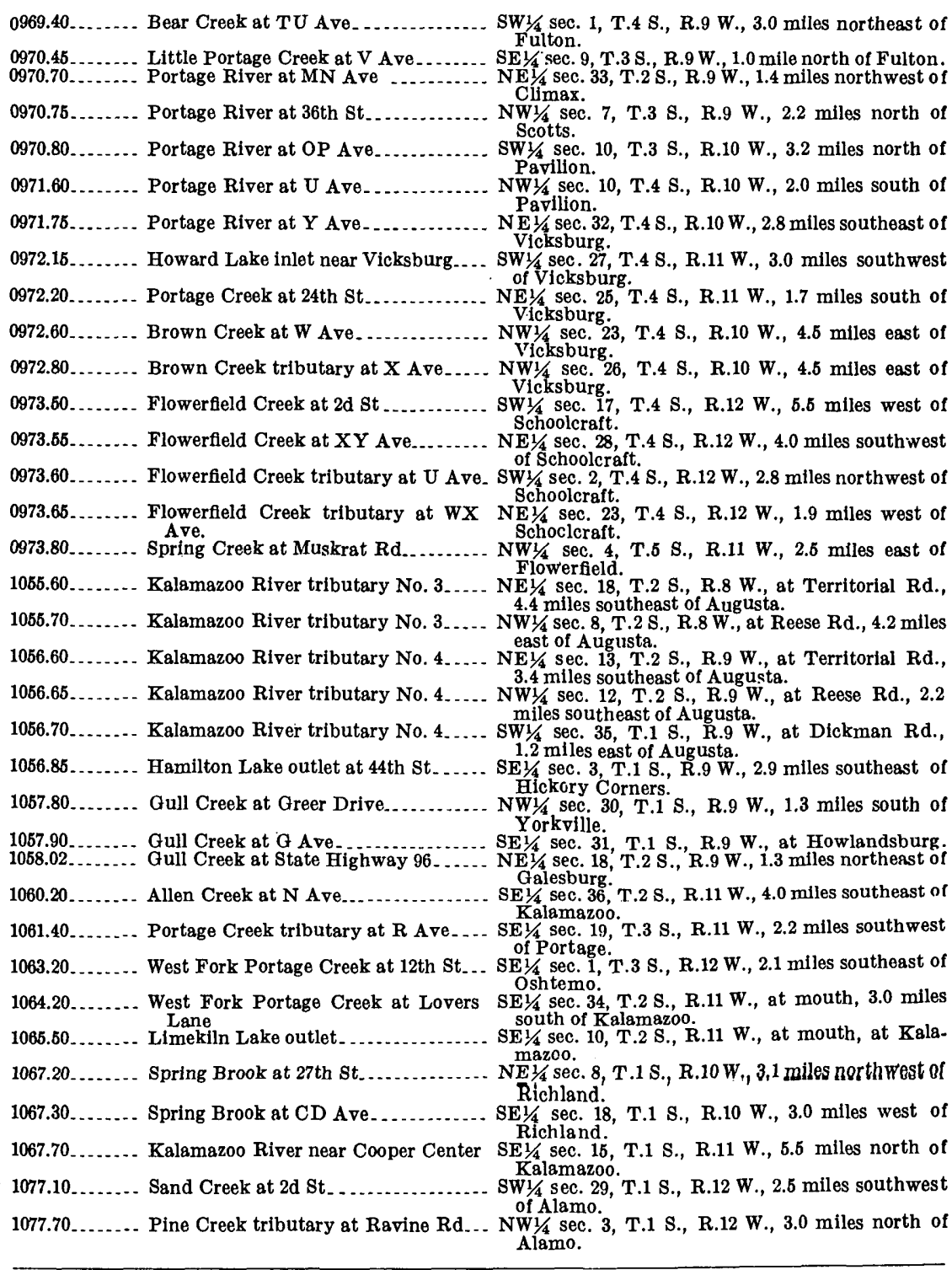


10-year recurrence intervals for miscellaneous sites to period 1931-65]

\begin{tabular}{|c|c|c|c|c|c|c|c|c|}
\hline \multirow{3}{*}{$\begin{array}{l}\text { Drainage } \\
\text { area } \\
\text { (sq mi) }\end{array}$} & \multicolumn{4}{|c|}{$\begin{array}{c}\text { Estimate of 7-day low flow at recurrence } \\
\text { interval shown }\end{array}$} & \multicolumn{4}{|c|}{$\begin{array}{c}\text { Estimate of 30-day low flow at recurrence } \\
\text { interval shown }\end{array}$} \\
\hline & \multicolumn{2}{|c|}{2 -yr } & \multicolumn{2}{|c|}{$10-\mathrm{yr}$} & \multicolumn{2}{|c|}{ 2-yr } & \multicolumn{2}{|c|}{$10-\mathrm{yr}$} \\
\hline & Cfs & $\begin{array}{l}\text { Cfs per } \\
\text { sq mi }\end{array}$ & Cfs & $\begin{array}{l}\text { Cis per } \\
\text { sq mi }\end{array}$ & Cfs & $\begin{array}{l}\text { Cfs per } \\
\text { sq mi }\end{array}$ & $\mathrm{Cfs}$ & $\begin{array}{l}\text { Crs per } \\
\text { sq mi }\end{array}$ \\
\hline 3.10 & 1.8 & 0.58 & 0.9 & 0.29 & 2.1 & 0.68 & 1.0 & 0.32 \\
\hline $\begin{array}{l}14.1 \\
5.04\end{array}$ & $\begin{array}{l}1.0 \\
0\end{array}$ & $0^{.07}$ & $0^{.26}$ & $0^{.02}$ & 1.3 & .09 & .31 & .02 \\
\hline 9.76 & 1.6 & .16 & 1.2 & .12 & 1.7 & .17 & 1.2 & .12 \\
\hline 16.9 & 1.3 & .08 & .53 & .03 & 1.7 & .10 & .74 & .04 \\
\hline 59.3 & 16 & .27 & 9.9 & .17 & 18 & .30 & 11 & .19 \\
\hline 70.6 & 17 & .24 & 11 & .16 & 18 & .25 & 11 & .16 \\
\hline 8.01 & .13 & .02 & .04 & .005 & .2 & .02 & .06 & .007 \\
\hline 53.9 & 23 & .43 & 15 & .28 & 25 & .46 & 16 & .30 \\
\hline 3.80 & .07 & .02 & .02 & .005 & .10 & .03 & .02 & .005 \\
\hline 4.65 & .56 & .12 & .32 & .07 & .64 & .14 & .36 & .08 \\
\hline 12.5 & 1.2 & .10 & .9 & .07 & 1.3 & .10 & 1.0 & .08 \\
\hline 18.9 & 3.6 & $\therefore$ & 2.9 & .15 & 3.8 & .20 & 3.0 & .16 \\
\hline 6.75 & 0 & 0 & 0 & 0 & - & - & & \\
\hline 11.9 & 0 & 0 & 0 & 0 & 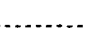 & & & . \\
\hline 10.9 & 2.8 & .26 & 2.0 & .18 & 2.9 & .27 & 2.2 & .20 \\
\hline 5.51 & .23 & .04 & .15 & .03 & .26 & .05 & .17 & .03 \\
\hline 6.41 & .13 & .02 & .04 & .006 & .18 & .03 & .05 & .008 \\
\hline 2.78 & 3.5 & 1.26 & 2.8 & 1.01 & 3.7 & 1.33 & 2.9 & 1.04 \\
\hline 4.14 & 4.7 & 1.14 & $3 . \dot{9}$ & .94 & 5. 0 & 1.21 & 4.1 & .99 \\
\hline 6.99 & 4.4 & .63 & 3.2 & .46 & 4.9 & .70 & 3.4 & .49 \\
\hline 7.69 & 1.4 & .18 & .8 & .10 & 1.7 & .22 & 1.0 & .13 \\
\hline 30.0 & 2.3 & .08 & 1.2 & .04 & 2.8 & .09 & 1.4 & .05 \\
\hline $\begin{array}{l}35.7 \\
39.9\end{array}$ & $\begin{array}{l}4.1 \\
2.6\end{array}$ & $\begin{array}{l}.11 \\
.07\end{array}$ & $\begin{array}{r}1.7 \\
.9\end{array}$ & $\begin{array}{l}.05 \\
.02\end{array}$ & $\begin{array}{l}\text { 5. } 2 \\
\text { 3. } 5\end{array}$ & $\begin{array}{l}.15 \\
.09\end{array}$ & $\begin{array}{l}2.2 \\
1.2\end{array}$ & $\begin{array}{l}.06 \\
.03\end{array}$ \\
\hline 11.2 & 1.4 & .12 & .7 & .06 & 1.5 & .13 & .8 & .07 \\
\hline 2.63 & 2.3 & .87 & 1.8 & .68 & 2.5 & .95 & 2.0 & .76 \\
\hline 13.0 & 6.3 & .41 & 3.3 & .25 & 5. 9 & .45 & 4.0 & .31 \\
\hline 21.7 & 2.7 & .12 & .5 & .02 & 3.8 & .18 & 1.0 & .05 \\
\hline 2. 58 & .18 & .07 & .15 & .06 & .20 & .08 & .16 & .06 \\
\hline 20.5 & .59 & .03 & .50 & .02 & .67 & .03 & .52 & .03 \\
\hline 22.4 & 3.8 & .17 & 3.4 . & .15 & 4.2 & .19 & 3.5 & .16 \\
\hline 1250 & 395 & .32 & 270 & .22 & 440 & .35 & 290 & .23 \\
\hline 21.2 & 6.4 & .30 & 5. 5 . & .26 & 7.1 & .33 & 5.8 & .27 \\
\hline 7.08 & .37 & .05 & .26 & .04 & .47 & .07 & .30 & .04 \\
\hline
\end{tabular}


national network of gaging stations in the U.S. Geological Survey's annual reports on surface-water supply. Numbers are assigned in ascending sequence in downstream order. Thus, numbers for locations in the headwaters of a basin are smaller than those for locations near the mouth. Numbers for locations on a tributary are intermediate between numbers for locations on the main stream above and below the tributary.

By knowing the flow characteristics at many sites throughout the county, the user can interpolate to his point of interest if he wants information quickly. Interpolation of the low-flow index, which varies mostly with geology, provides only a very rough estimate of the low flow to be expected. For more reliable information at a site not listed, it is necessary to obtain a few base-flow measurements. These should be made on two or more recessions and, if time permits, during different seasons and different years. These measurements can be related to concurrent discharges at one or more gaging stations for which low-flow frequency curves have been developed. Discharges corresponding to certain recurrence intervals at the gaging station can then be transferred through the relation curve to obtain corresponding discharge at the miscellaneous site.

\section{STORAGE ANALYSIS}

When the low flow of a stream is insufficient to meet the demands placed upon it, it becomes necessary to supplement the flow with water obtained from storage. Storage requirements needed to meet the demands placed on the stream may be determined by analyzing the stream's low-flow records. In this analysis, low-flow frequency curves as shown in figure 10 are developed for the gaging station. These curves are used to define frequency-mass curves (volume available without storage), from which storage requirements to meet selected draft rates can be determined. Figure 11 is an example of a frequencymass relationship prepared from low-flow frequency data at the 20 year recurrence interval contained in the family of curves in figure 10. The curve represents the minimum total runoff available corresponding to a 20-year recurrence interval and is obtained by plotting the volume of runoff for various durations of minimum flow against the duration period.

Figure 11 shows that a storage volume of 13,500 cfs-days or about 27,000 acre-ft would be needed to provide a draft rate of $400 \mathrm{cfs}$. This storage would fail to provide this draft rate on the average of once in 20 years. Suppose it would be desirable to maintain a draft rate of $600 \mathrm{cfs}$ at Comstock. A storage volume of 69,000 cfs-days or about 138,000 acre-ft would be needed. The curve indicates that, to maintain this draft rate, storage for more than 1 year is required. 


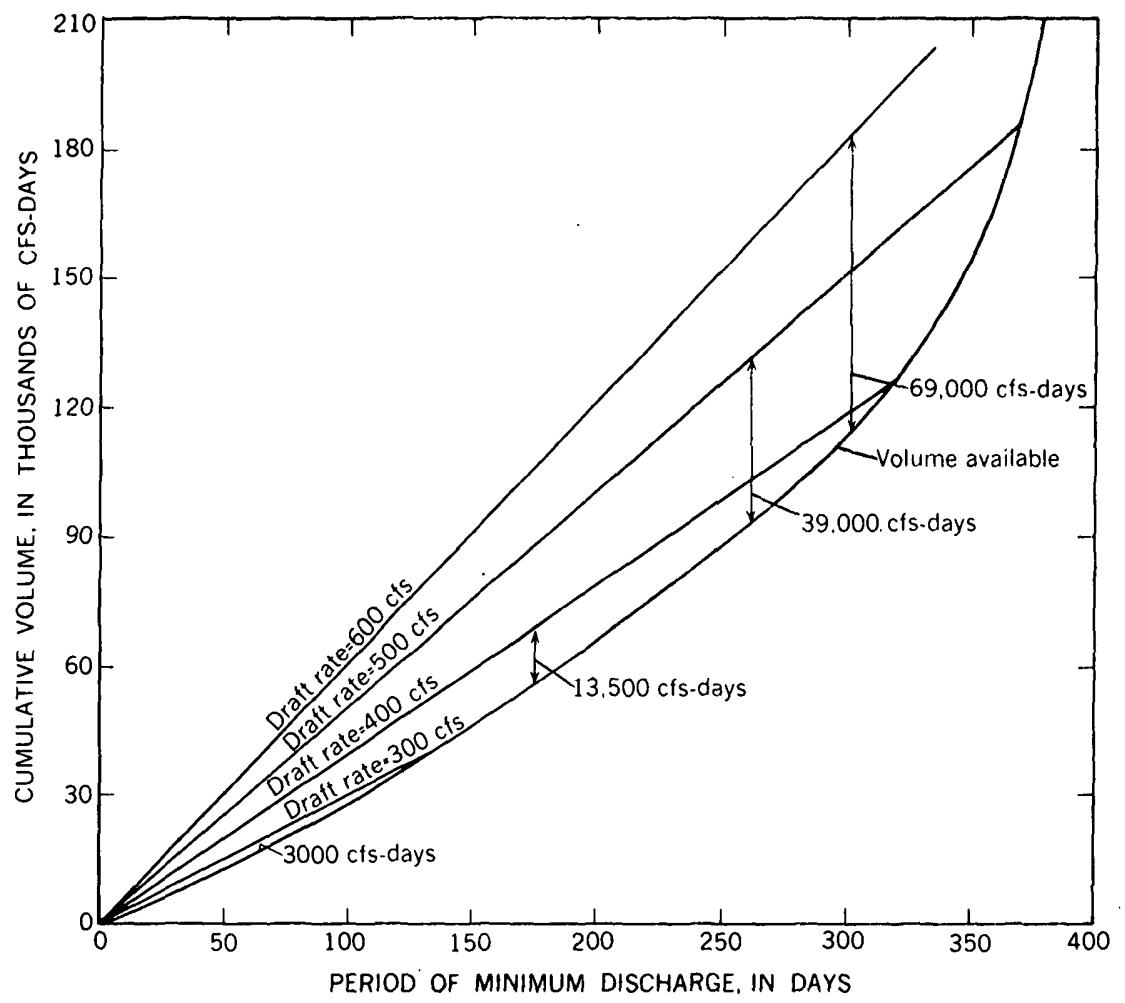

FiguRE 11.-Frequency-mass curve and draft-storage lines for 20-year recurrence interval, Kalamazoo River at Comstock. The storage required for each draft rate is the maximum interval between the line representing the draft rate and the curve representing the volume available.

Storage requirements to maintain streamflow may be determined for other stations having long-term records, and has been outlined for Comstock above. However, kecause of the limited data available on most streams in Kalamazoo County, a regional analysis was necessary to determine storage needs. In this analysis, storage requirements for various draft rates are related to a low-flow index of each stream, using a method described by H. C. Riggs (written commun., 1964).

The low-flow index used in this report is the median 7-day annual low flow. This flow is defined as the lowest average flow for 7 consecutive days occurring at an average interval of 2 years. Therefore, flows equal to or less than the 7-day low flow can be expected to occur on an average of once every 2 years. Values of 7 -day low flows at many sites in the county are shown in tables 9 and 10 and on plate 5 .

Draft-storage frequency analyses for 21 stations in southwestern Michigan were used to develop regional relationships applicable only for within-year storage. These relationships are a valuable tool for 


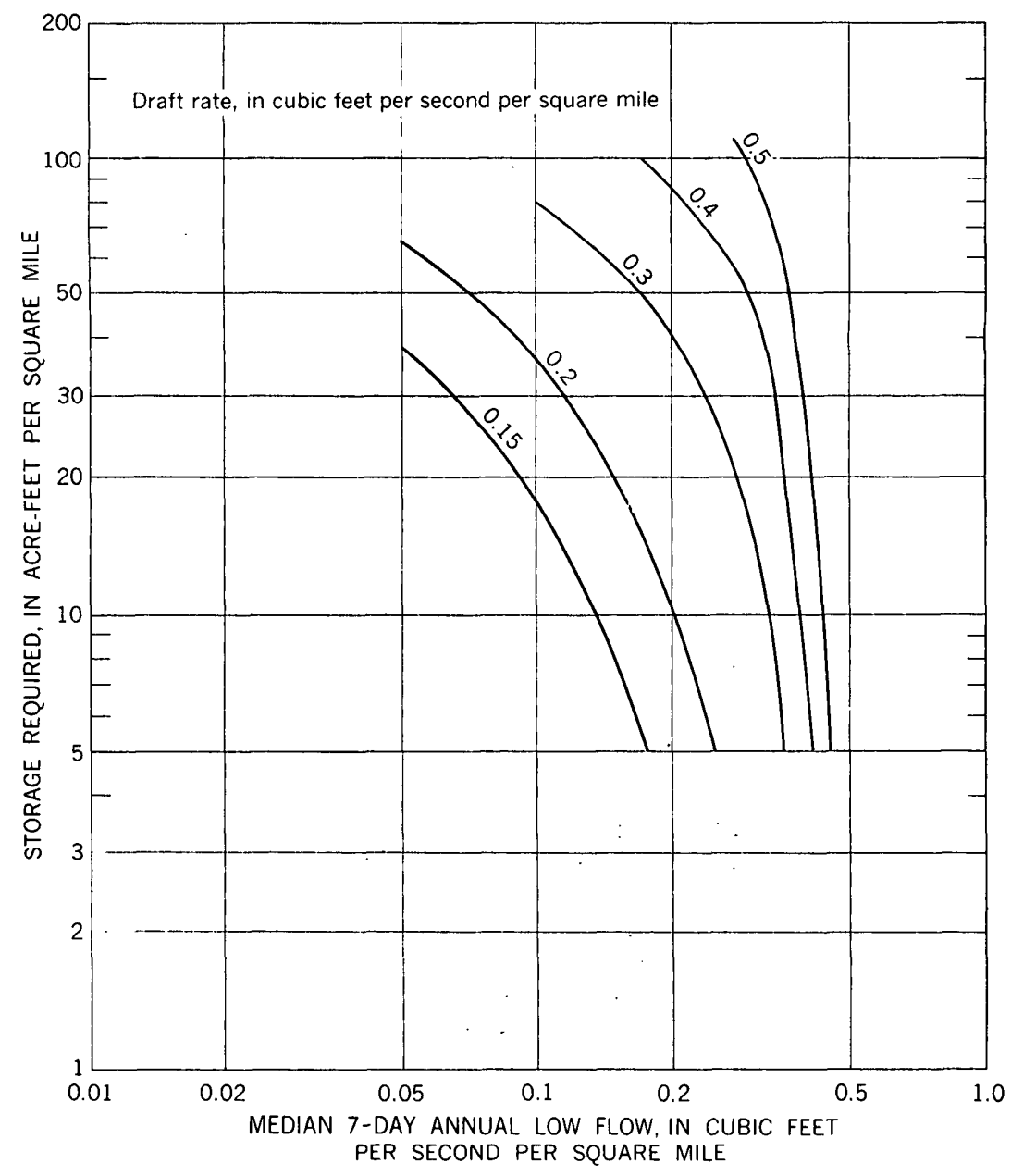

Figure 12.-Regional draft-storage curves for the 20-year recurrence interval for southwestern Michigan. To determine the storage required for a given draft rate, enter the graph on the bottom scale at the value indicated for the median (2-year recurrence interval) 7-day annual low flow at the designated station (tables 9 and 10). Read up from this point on a vertical line to the point of intersection with the curve representing the desired draft rate. Follow a horizontal line from this point of intersection to the scale at the left of the graph. The horizontal line intersects the scale at the value of the storage required.

preliminary investigations of storage requirements. Curves for 20year recurrence interval indicate a 5-percent chance that the storage volume would not support the draft rate (fig. 12). Regional draftstorage curves for 10-year recurrence intervals were also developed 


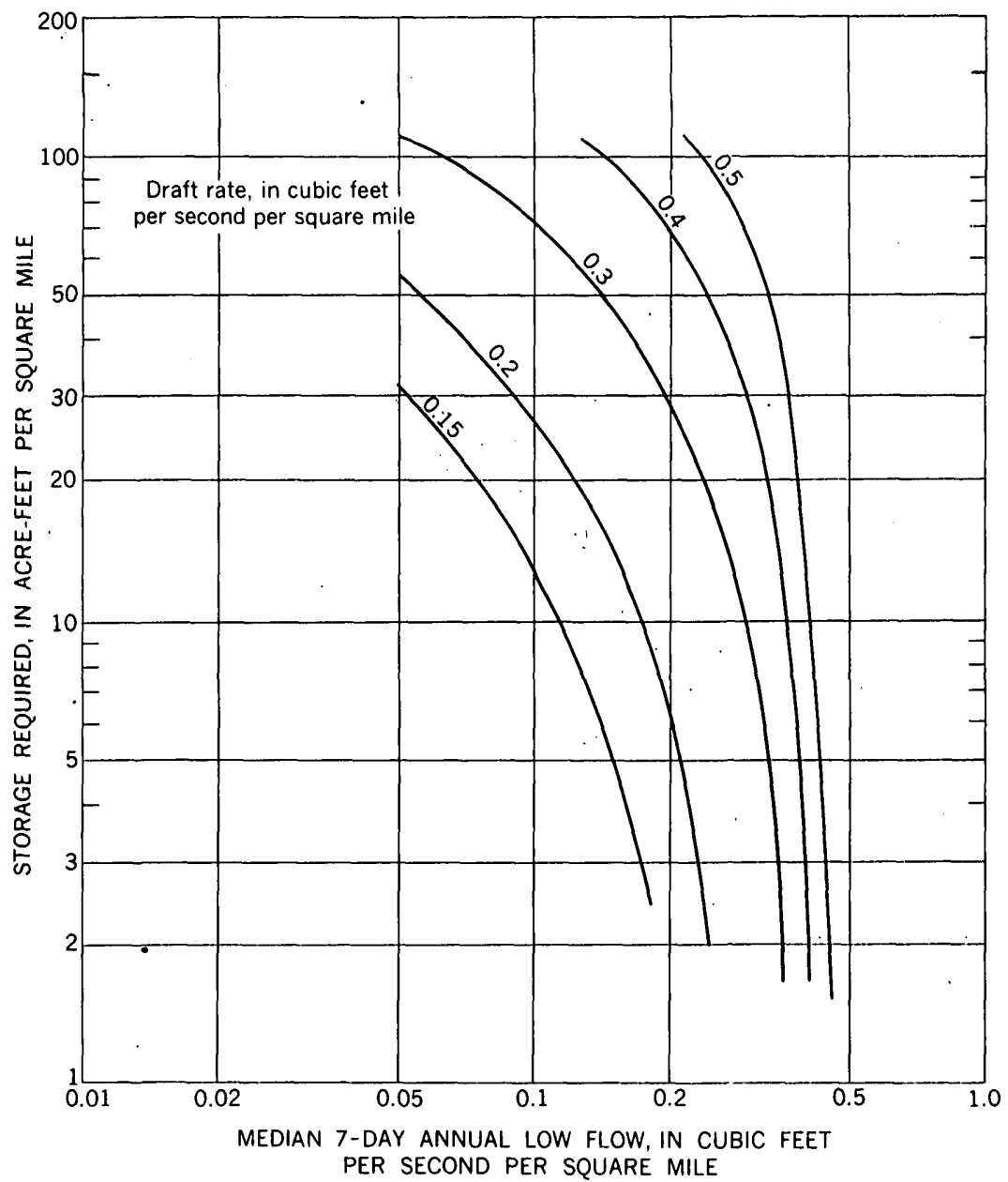

Froure 13.-Regional draft-storage curves for the 10-year recurrence interval for southwestern Michigan.

for those users willing to risk a 10-percent chance of storage not supporting a required draft rate (fig. 13).

For some stations listed in table 9 , the average flow is less than 0.5 cfs per sq mi. Since the average flow is the ultimate limit of development, figures 12 and 13 cannot be used for draft rates exceeding or approaching the average flow of a stream.

Storage requirements for any site where the low-flow index is known can be estimated from the regional draft-storage curves. Exceptions, however, are those streams having an unusually high lowflow index. In Kalamazoo County, streams in this category are 
Augusta Creek, Portage Creek near Portage, Gourdneck Creek, and Rupert Lake outlet. The low-flow index for these streams exceeds the highest draft rates used in the regional analysis. The median 7-day low flow for these stations average about 60 percent of the average discharge. Therefore, with storage, draft rates approaching the average discharge (maximum available supply) of each of these streams could be developed. Continual draft rates cannot be greater than average discharge, regardless of volume of storage.

Suppose a user needs 5.5 cfs at Comstock Creek near Kalamazoo and can tolerate inadequate supplies on an average of once in 20 years. The required draft rate would be $0.3 \mathrm{cfs}$ per sq $\mathrm{mi}(5.5 \div 18.3)$. From table 9 the low-flow index for Comstock Creek is $3.3 \mathrm{cfs}$ or $0.18 \mathrm{cfs}$ per sq mi $(3.3 \div 18.3)$. Using the low-flow index of $0.18 \mathrm{cfs}$ per sq $\mathrm{mi}$ and the draft rate of $0.3 \mathrm{cfs}$ per sq $\mathrm{mi}$ in figure 12, storage of about 860 acre-ft ( 47 acre-ft per sq mi) is needed to maintain a draft rate of $0.3 \mathrm{cfs}$ per sq mi or $5.5 \mathrm{cfs}$ at this location. A draft rate of $0.4 \mathrm{cfs}$ per sq mi cannot be realized since it exceeds the average discharge of $0.36 \mathrm{cfs}$ per sq mi. Such information will aid the water manager in his decisions concerning water needs from this creek.

Draft rates shown do not take into account water lost by evaporation, seepage, or conveyance losses between storage site and point of utilization; thus the amount of storage required for a given draft rate must be increased to allow for these losses. These losses are influenced by the area and depth of the storage reservoir and the character of the geologic materials at the reservoir site. They must be determined individually for each site.

\section{FLOODS}

Floods were not of primary interest in this study. However, the magnitude and frequency of floods are essential elements in studies involving flood-control design or the economics involved in the design of such structures as dams, culverts, and bridges.

Most floods in the county occur in the spring as a result of snowmelt and spring rains in combination with frozen or saturated soils. The maximum flood of record for Kalamazoo River at Comstock occurred on April 8, 1947. The river rose to an altitude of 767.06 feet above mean sea level and had a peak discharge of $6,910 \mathrm{cfs}$. This was the greatest flood since 1904 and 1908, when the annual flood peaks may have been higher.

By arraying annual flood peaks in order of magnitude and computing a recurrence interval to each peak, a flood-frequency curve can be developed for a gaging station. Figure 14 shows the floodfrequency relationship for Kalamazoo River at Comstock. 


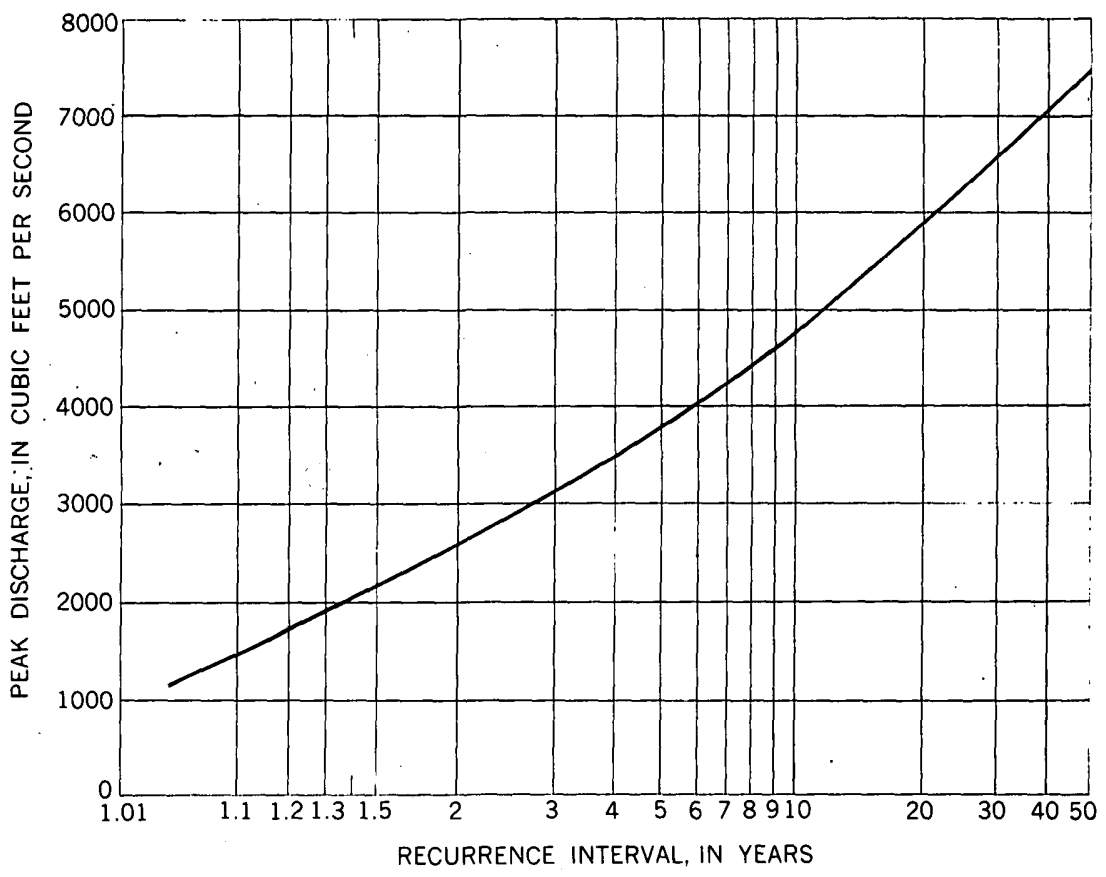

Figure 14.-Flood-frequency curve for Kalamazoo River at Comstock, 1931-67.

Other gaging stations within the county have very short periods of record. Therefore, regional flood-frequency relations must be used to determine the magnitude of floods for selected frequencies at these sites and for ungaged streams. The regional relationships were developed in a report on the magnitude and frequency of floods in the St. Lawrence River basin (Wiitala, 1965) and are applicable to unregulated streams draining more than $30 \mathrm{sq} \mathrm{mi}$. In that report several flood-frequency regions were delineated, two of which are applicable to Kalamazoo County (fig. 15). Estimates of flood frequencies as applied to these regions involve the use of two curves-the relation between size of drainage area and magnitude of the mean annual flood (fig. 16), and the relation between recurrence interval and discharge, in ratio to mean annual flood (fig. 17). Thus, although flood data are lacking in the county, the relationships expressed in the curves afford a means of making estimates of flood magnitude and frequency.

To illustrate the use of these curves, suppose it is desired to know the magnitude of a flood having a 30-year recurrence interval for Augusta Creek gaging station. The drainage area at this site is 38.9 sq. mi. Using this value in figure 16 gives a mean annual flood of $140 \mathrm{cfs}$. The site is located in area $\mathrm{E}$; therefore, the ratio of the 30 year flood to the mean annual flood is 2.4. Multiplying this ratio by the mean annual flood gives a 30-year flood magnitude of about $340 \mathrm{cfs}$. 


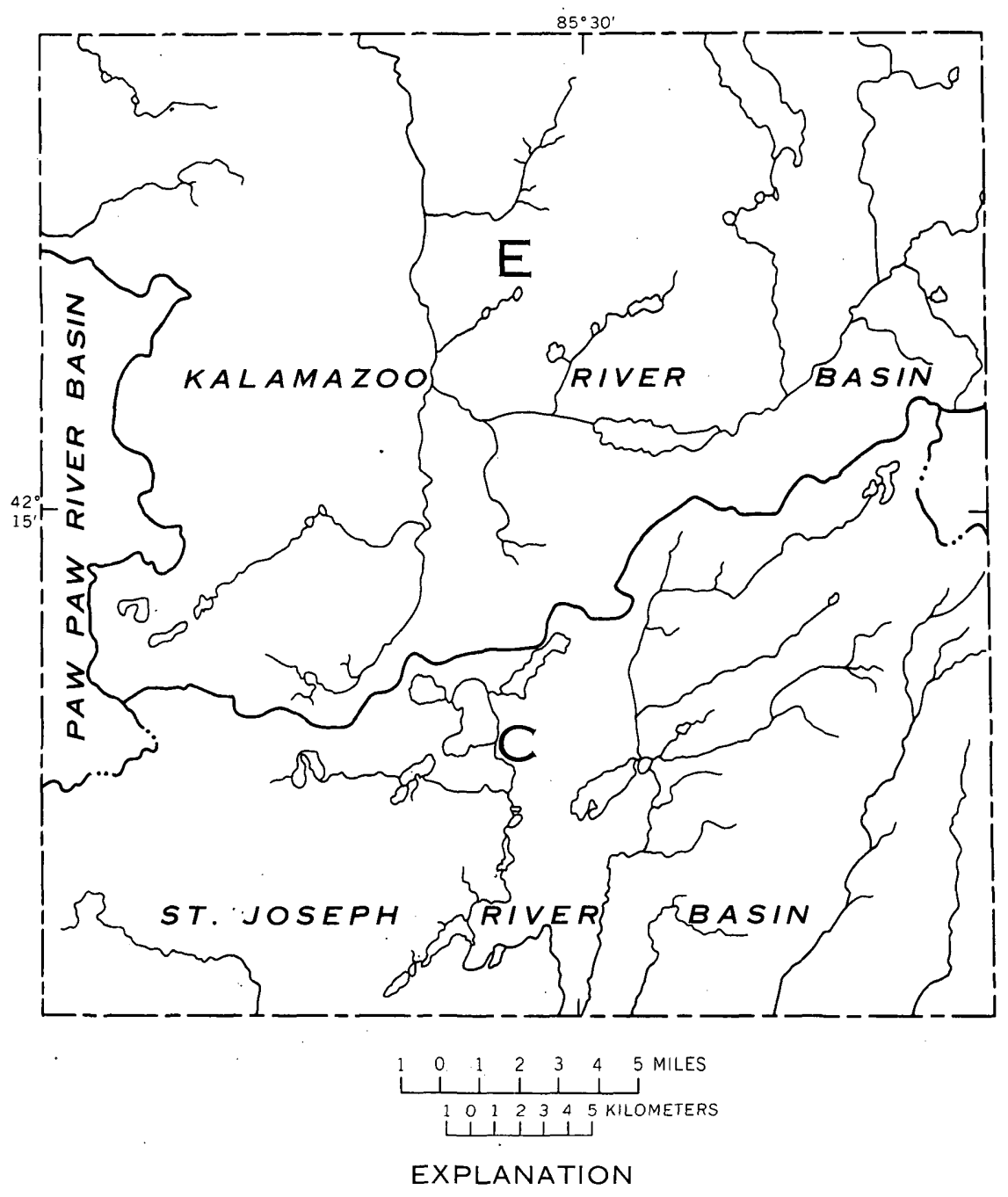

Basin boundaries $\quad \overline{\text { Regional boundaries }}$

Figure 15.-Flood-frequency regions $\mathrm{C}$ and E, applicable to Kalamazoo County (from Wiitala, 1965).

\section{CHEMICAL QUALITY}

The natural chemical quality of streams in the county is dependent upon the ratio of ground-water runoff to the surface runoff. During low flow, when the water is of ground-water origin, dissolved solids are generally more concentrated than during periods of high flow, when much of the water is direct precipitation and surface runoff. 


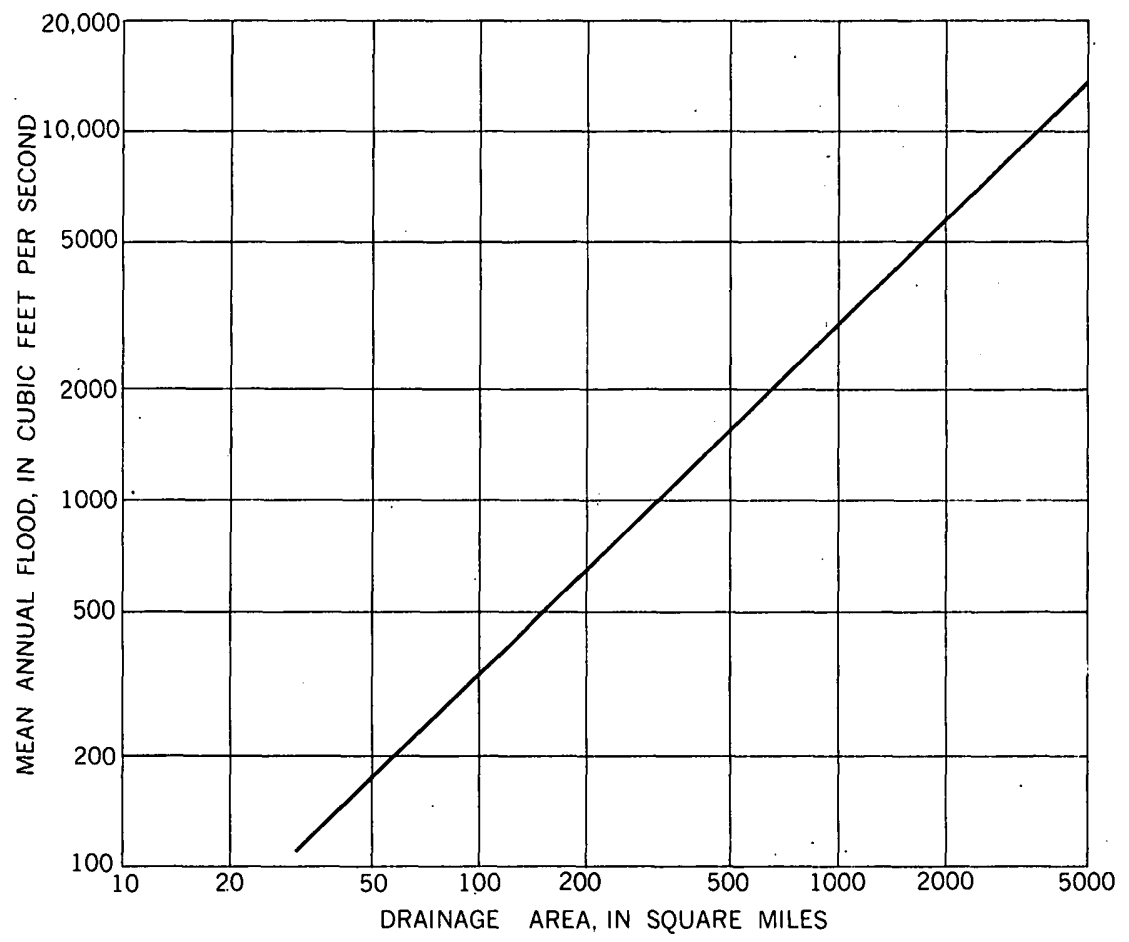

Frgure 16.-Variation of mean annual flood with drainage area (from Wiitala, 1965).

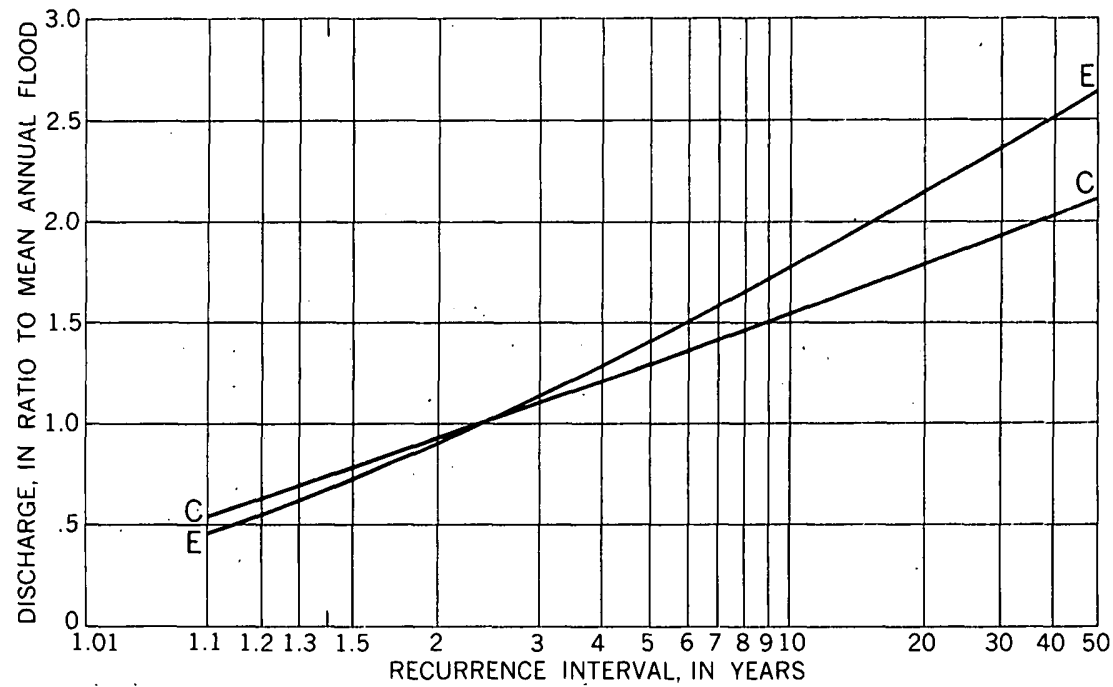

FIGURE 17.-Frequency of annual floods, flood-frequency regions $\mathrm{C}$ and $\mathrm{E}$ (from Wiitala, 1965). 
The relationship between stream discharge and dissolved solids is illustrated for several sites in the county on plate 6 . The presence of lakes in the stream network has a modifying effect on the dissolved solids-discharge relationship. The range of dissolved solids is reduced resulting in a flattening of the dissolved solids-discharge curves.

West Fork Portage Creek is an exception in that dissolved solids increase with increasing discharge. The reversal of the normal condition in this stream results from the combination of many lakes in the headwaters which capture and store direct precipitation and the lack of significant ground-water inflow throughout most of the lower reach. The water, therefore, contains less dissolved solids under lowflow conditions than other rivers in the area and consequently' is more readily modified by surface runoff.

Nitrate and phosphate values usually increase in concentration with an increase in surface runoff. This is due primarily to solution of nutrients from agricultural fertilizer and decaying vegetation on the land surface being washed into the streams.

Urbanization can also alter the dissolved-solids-discharge relationship. The presence of soluble minerals from smoke ash, lawn fertilizer, sanitary landfill, industrial stock piles, and other products of urban development can cause changes in the relationship between streamflow and quality of water.

Chemical analyses of water samples collected during the period 1964-66 are summarized in table 11. Significant constituents are presented graphically on plate 6 . The plate shows that sulfate concentrations are slightly higher in streams in the southeastern part of the county than in other streams. These higher sulfate values are attributed to solution of gypsum or anhydrite in the clay till that is at or near the surface in this area. These sulfate minerals probably influence the formation of the many marl deposits, as the minerals could cause supersaturation of calcium carbonate (marl) in the water.

Natural chloride concentration in the streams is low (table 11 and pl. 6). Consequently, values greater than about $10 \mathrm{mg} / \mathrm{l}$ suggest the presence of wastes. On this basis, several samples collected at one or more localities on Portage Creek (Kalamazoo basin), Kalamazoo River, Allen Creek, Portage Creek (St. Joseph basin), and Limekiln Lake outlet indicate contamination. One source of chloride contamination is the salt applied to roads for ice and dust control.

\section{WITHDRAWALS}

A survey of water withdrawn from the rivers and streams by industries during 1966 showed that about $58 \mathrm{mgd}$ was withdrawn. There is little consumptive use of this water. Most of the water withdrawn is 
returned to the rivers and streams and again becomes available to users downstream, although the physical and (or) chemical quality may be degraded. Figure 18 illustrates that present withdrawal of water is significant on some streams but is only a small percentage of that available in others. In the illustration, 7-day $Q_{2}$, which is the average 7-day low flow having a recurrence interval of 2 years, is used as a measure of the dependable supply of water from the streams.

At present only a small percentage of the dependable supply in the Kalamazoo River is withdrawn. Low flows for Portage Creek (Kalamazoo basin) and Gourdneck Creek are augmented by water released from storage ponds to meet the demands placed on the streams by industry. The dependable supply for Portage Creek was determined by adding the 7-day $Q_{2}$ flow at the mouth of West Fork Portage Creek and the 7-day $\mathrm{Q}_{2}$ flow at Portage Creek near Portage. The flow in Portage Creek is further augmented by water pumped from the ground for industrial use and discharged to the stream. Due to the variability in ground water pumped and discharged to the stream, only the natural flow was used in defining the dependable supply of Portage Creek. The dependable supply determined for Gourdneck Creek is low because the values of 7-day $Q_{2}$ at the partialrecord site were not adjusted for diversion of water to West Lake from the headwaters of Gourdneck Creek. Without diversion the supply would be higher. Water from Gourdneck Creek is stored in Sunset Lake for use by industry in Vicksburg.

The benefits of storage in the Gourdneck Creek basin and in the lower reaches of Portage Creek (near Kalamazoo) are apparent from figure 18 . The present withdrawal exceeds the 7-day $Q_{2}$; however, in times of low flow, storage from Sunset Lake and from the small ponds

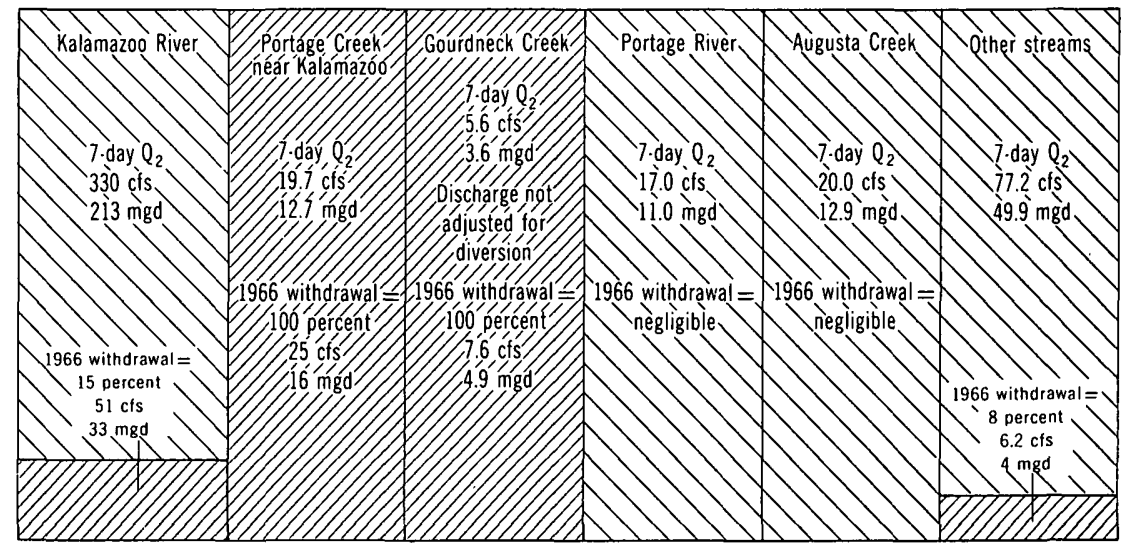

FraURe 18.-Comparison of surface-water withdrawal in 1966 with dependable supply (7-day $Q_{2}$ ). 
TABLE 11.-Chemical quality of

[Chemica] analyses in milligrams per liter except

\begin{tabular}{|c|c|c|c|c|c|c|c|c|c|c|c|}
\hline Station & Stream and locality & $\begin{array}{l}\text { Date of } \\
\text { collec- } \\
\text { tion }\end{array}$ & $\begin{array}{c}\text { Dis- } \\
\text { charge } \\
\text { (efs) }\end{array}$ & 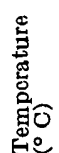 & 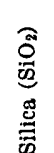 & 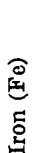 & 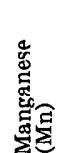 & 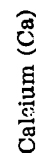 & & & 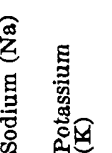 \\
\hline
\end{tabular}

\begin{tabular}{|c|c|}
\hline $\begin{array}{l}0969.40 \ldots \\
0969.50 \ldots\end{array}$ & $\begin{array}{l}\text { Bear Creek at TU Ave..... } \\
\text { Bear Creek near Fulton.... }\end{array}$ \\
\hline 0970.40 & $\begin{array}{l}\text { Little Portage Creek near } \\
\text { Climax }\end{array}$ \\
\hline 0970.45 & $\begin{array}{l}\text { Little Portage Creek at } \\
\text { V Ave }\end{array}$ \\
\hline 0970.50.. & Little Portage Creek \\
\hline 0970.60 & $\begin{array}{l}\text { tributary at V Ave } \\
\text { Little Portage Creek near } \\
\text { Fulton. }\end{array}$ \\
\hline
\end{tabular}

10-19-65 6- 2-64 2-18-65 8-11-65 6- 2-64 2-18-65 8-12-65 10-19-65 10-19-65 2-18-65 8-12-65 10-19-65 $10-19-65$ 0970.75 . . Portage River at 36th St_ . . 10-18-65 0970.80.. Portage River at OP Ave ... 10-18-65 0971.15.. Portage River tributary near McKain Corners ..... 6- 6-2-64 0971.20_. Portage River near Pavilion $\begin{array}{r}2-18-65 \\ 8-5-65 \\ 6-2-64\end{array}$ $2-18-65$ $8-5-65$

0971.30_. Dorrance Creek at McKain Corners............... 6-2-64 2-18-65

0971.60.. Portage River at U Ave... . $\begin{array}{r}8-6-19-65 \\ 10-65\end{array}$ 0971.70.. Portage River near

Vicksburg

2-18-65 8- 6-65 10-18-65 $12-8-66$

0971.75 _. Portage River at Y Ave ... 10-18-65 0971.95 . - Gourdneck Canal near Schoolcraft................ 10-19-65 0972.00.. Gourdneck Creek near Schoolcraft.

$2-18-65$ $8 x+40$

0972.05. Gourdneck Creek near Vicksburg.

$10-19-65$

6- 2-64 $2-19-65$ $8-5-65$

0972.07 _ Austin Lake outlet at

TU Ave.

$12-8-66$ 0972.10.. Portage Creek at W Ave... 8- 5-65 0972.15 . . Howard Lake inlet near Vicksburg.............. 6-2-65 $2-19-65$ 8- 6-65

0972.20. . Portage Creek at 24th St . . 10-19-65 0972.40 _ - Portage Creek near Mendon. 10-19-65 0972.60.. Brown Creek at W Ave . 10-19-65 0972.80.. Brown Croek tributary at 10-19-65

X Ave.
0973.30.. Brown Creek near Vicksburg. $2-18-65$

0973.50 Flowerfield Creek at '2d St 8- 5-65 0973.55.. Flowerfield Creek at XY $10-18-65$ 0973.70_. Flowerfield Creek at Flowerfield.

6- $2-64$

2-19-65 $8-12-65$ $8-12-60$
$12-8-66$
$2.27 \quad 12.2$

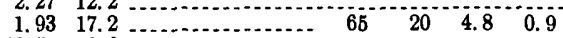

$10.7 \quad 0.6$

$1.70 \quad 24.4 \quad \ldots$.

$\begin{array}{rrrrrrr}49 & 19.4 & \ldots & \ldots & \ldots & & \end{array}$

$6.86 \quad 2.2$

$.56 \quad 29.4$

$2.84 \quad 13.3$

1.49

$\begin{array}{lllllllll}\ldots & 2.8 & 8.6 & 0.37 & 0.02 & 82 & 23 & 4.1 & .6\end{array}$

$5.02 \quad 18.3$

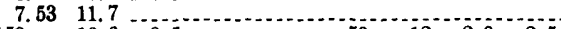

$158 \quad 10.69 .5 \ldots 50 \quad 12 \quad 2.0 \quad 2.5$

2.1616 .7

$2.70 \quad 16.1 \quad 110.0$

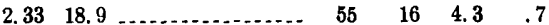

$\begin{array}{lr}\text { 5. } 06 & 2.7 \\ \text { 3. } 56 & 26.7 \\ 8.46 & 13.9\end{array}$

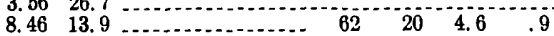

$21.9 \quad 3.3$. $3 . \ldots \ldots \ldots \ldots$

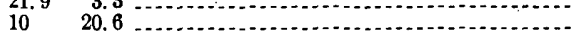

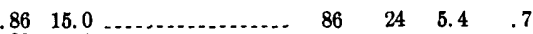

$5.88 \quad 1.1$

$25 \quad 23.3$

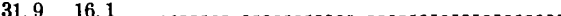

$\begin{array}{lllllllll} & 1.7 & 6.1 & .14 & .00 & 62 & 17 & 3.6 & 1.2\end{array}$

10.8 26.1 $2.1 . \ldots \ldots$

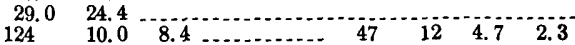

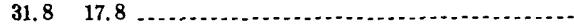

3. 6916.7

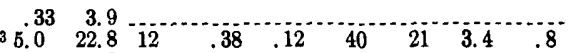

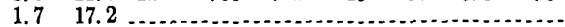

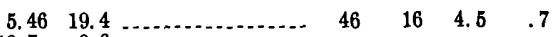

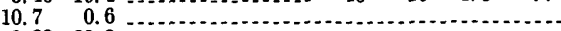

3. 8822.2 (2)

$22.0 \quad 9.4 \quad \ldots$

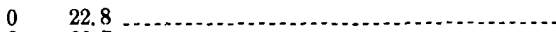

$8 \quad 26.7$

$\begin{array}{lllllll}10 & 17.2 & 54 & 16 & 3.3 & .4\end{array}$

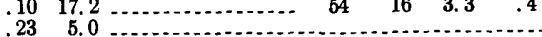

.3020 .00

41.4 16. 1

$41.8 \quad 15.6$

(1)

(1)

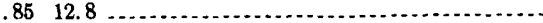

$\begin{array}{llllllll}2.62 & 20 & \ldots & \ldots & \ldots & \ldots & & \end{array}$

$11.6 \quad 2.2$

1.7219 .4

$1.53 \quad 15.0$

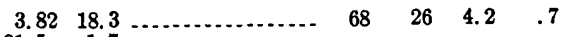

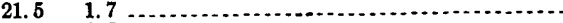

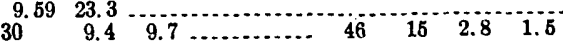

See footnotes at end of table. 


\section{water in streams}

$\mathrm{pH}$, color, or where indicated otherwise]

\begin{tabular}{|c|c|c|c|c|c|c|c|c|c|c|c|c|}
\hline \multirow{2}{*}{ 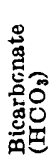 } & \multirow{2}{*}{ 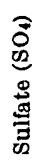 } & \multirow{2}{*}{ 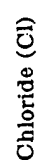 } & \multirow{2}{*}{ 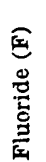 } & \multirow{2}{*}{ 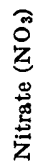 } & \multirow{2}{*}{ 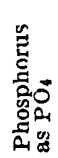 } & \multirow{2}{*}{$\begin{array}{l}\text { Dissolved } \\
\text { solids } \\
\text { (residue } \\
\text { on evap- } \\
\text { oration at } \\
180^{\circ} \mathrm{C} \text { ) }\end{array}$} & \multirow{2}{*}{$\begin{array}{l}\text { Loss } \\
\text { on } \\
\text { igni- } \\
\text { tion }\end{array}$} & \multicolumn{2}{|c|}{$\begin{array}{l}\text { Hardness } \\
\text { as } \mathrm{CaCO}_{3}\end{array}$} & \multirow{2}{*}{$\begin{array}{l}\text { Specifle } \\
\text { conduct- } \\
\text { ance (mi- } \\
\text { cromhos } \\
\text { at } 25^{\circ} \mathrm{C} \text { ) }\end{array}$} & \multirow[b]{2}{*}{$\mathrm{pH}$} & \multirow[b]{2}{*}{$\begin{array}{l}\text { Color } \\
\text { (Pt-Co } \\
\text { scale) }\end{array}$} \\
\hline & & & & & & & & $\begin{array}{c}\text { Calcium, } \\
\text { mag- } \\
\text { nesium }\end{array}$ & $\begin{array}{l}\text { Non- } \\
\text { carbon- } \\
\text { ate }\end{array}$ & & & \\
\hline
\end{tabular}

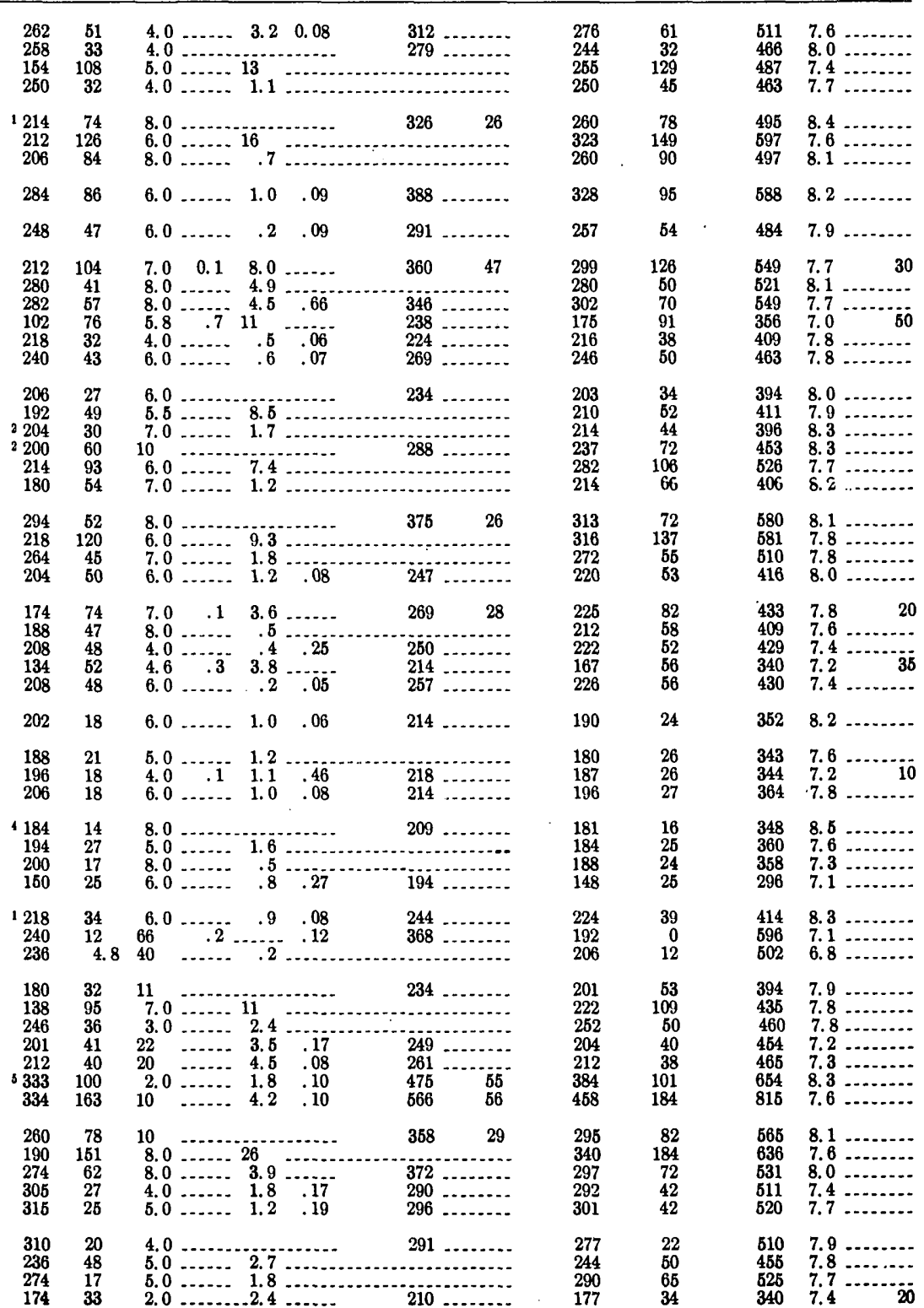


TABLE 11:-Chemical quality of

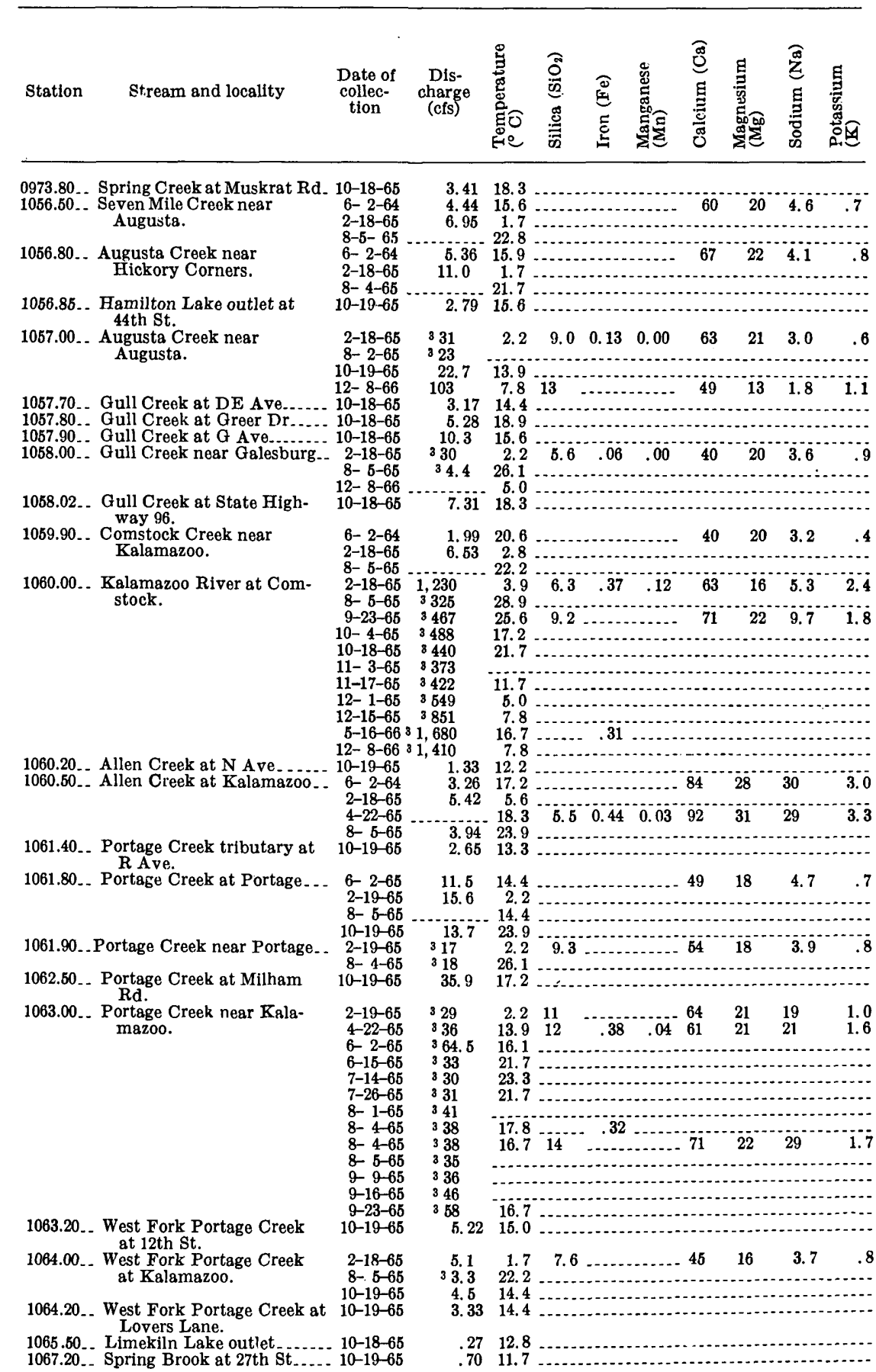

See footnotes at end of table. 
water in streams-Continued

\begin{tabular}{|c|c|c|c|c|c|c|c|c|c|c|c|c|}
\hline \multirow{2}{*}{ 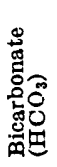 } & \multirow{2}{*}{ 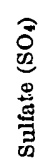 } & \multirow{2}{*}{ 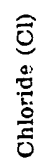 } & \multirow{2}{*}{ 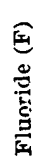 } & \multirow{2}{*}{ 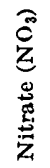 } & \multirow{2}{*}{ 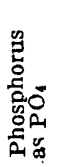 } & \multirow{2}{*}{$\begin{array}{l}\text { Dissolved } \\
\text { solids } \\
\text { (residue } \\
\text { on evap- } \\
\text { oration at } \\
180^{\circ} \mathrm{C} \text { ) }\end{array}$} & \multirow{2}{*}{$\begin{array}{c}\text { Loss } \\
\text { on } \\
\text { igni- } \\
\text { tion }\end{array}$} & \multicolumn{2}{|c|}{$\begin{array}{l}\text { Hardness } \\
\text { as } \mathrm{CaCO}_{3}\end{array}$} & \multirow{2}{*}{$\begin{array}{l}\text { Specific } \\
\text { conduct- } \\
\text { ance (mi- } \\
\text { cromhos } \\
\text { at } 25^{\circ} \mathrm{C} \text { ) }\end{array}$} & \multirow[b]{2}{*}{$\mathrm{pH}$} & \multirow[b]{2}{*}{$\begin{array}{c}\text { Color } \\
\text { (Pt-Co } \\
\text { scale) }\end{array}$} \\
\hline & & & & & & & & $\begin{array}{c}\text { Calcium, } \\
\text { mag- } \\
\text { nesium }\end{array}$ & $\begin{array}{l}\text { Non- } \\
\text { carbon- } \\
\text { ate }\end{array}$ & & & \\
\hline
\end{tabular}

\begin{tabular}{|c|c|c|c|c|c|c|c|c|c|c|}
\hline $\begin{array}{r}1230 \\
266 \\
242 \\
276 \\
282 \\
248 \\
282 \\
270\end{array}$ & $\begin{array}{l}31 \\
17 \\
30 \\
17 \\
23 \\
37 \\
27 \\
12\end{array}$ & $\begin{array}{l}4.0 \\
6.0 \\
5.0 \\
4.0 \\
6.0 \\
4.0 \\
6.0 \\
2.0\end{array}$ & $\begin{array}{l}- \\
- \\
- \\
- \\
- \\
- \\
-\end{array}$ & \begin{tabular}{r}
2.2 \\
\hdashline 1.7 \\
.9 \\
\hdashline 5.9 \\
5.2 \\
.8
\end{tabular} & $\begin{array}{l}.07 \\
\cdots \\
0.0 \\
.06\end{array}$ & \begin{tabular}{c}
253 \\
251 \\
\hdashline 284 \\
\hdashline 264
\end{tabular} & $\begin{array}{l}232 \\
232 \\
226 \\
252 \\
258 \\
252 \\
272 \\
237\end{array}$ & $\begin{array}{l}37 \\
14 \\
28 \\
26 \\
26 \\
48 \\
40 \\
16\end{array}$ & $\begin{array}{l}430 \\
433 \\
427 \\
456 \\
483 \\
467 \\
495 \\
428\end{array}$ & $\begin{array}{l}8.3 \\
8.1 \\
8.1 \\
8.1 \\
8.1 \\
8.2 \\
8.2 \ldots \\
7.9 \\
8.0 \\
7.5 \\
7.5\end{array}$ \\
\hline $\begin{array}{r}248 \\
262 \\
282 \\
174 \\
178 \\
210 \\
236 \\
186 \\
2216 \\
176 \\
242\end{array}$ & $\begin{array}{l}33 \\
24 \\
23 \\
31 \\
22 \\
20 \\
22 \\
23 \\
20 \\
28 \\
22\end{array}$ & $\begin{array}{l}4.0 \\
6.0 \\
4.0 \\
2.0 \\
6.0 \\
7.0 \\
7.0 \\
6.0 \\
6.0 \\
6.5 \\
7.0\end{array}$ & 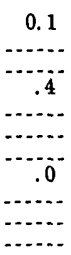 & $\begin{array}{r}2.9 \\
3.1 \\
2.6 \\
2.6 \\
.5 \\
.6 \\
.7 \\
.4 \\
1.2 \\
.8 \\
.6\end{array}$ & $\begin{array}{l}.08 \\
.06 \\
06 \\
06\end{array}$ & $\begin{array}{r}256 \\
273 \\
228 \\
170\end{array}$ & $\begin{array}{l}244 \\
250 \\
260 \\
176 \\
168 \\
196 \\
220 \\
182 \\
208 \\
170 \\
226\end{array}$ & $\begin{array}{l}40 \\
36 \\
28 \\
33 \\
22 \\
24 \\
26 \\
30 \\
28 \\
26 \\
28\end{array}$ & $\begin{array}{l}441 \\
463 \\
475 \\
343 \\
333 \\
370 \\
409 \\
342 \\
385 \\
334 \\
415\end{array}$ & 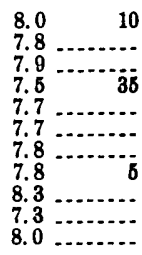 \\
\hline $\begin{array}{r}194 \\
184 \\
204 \\
150 \\
208 \\
1232 \\
234 \\
265 \\
219 \\
210 \\
202 \\
251 \\
216 \\
226 \\
314 \\
316 \\
292 \\
276 \\
316 \\
214\end{array}$ & $\begin{array}{r}23 \\
28 \\
23 \\
83 \\
48 \\
49 \\
50 \\
54 \\
52 \\
52 \\
51 \\
65 \\
66 \\
73 \\
70 \\
57 \\
95 \\
108 \\
51 \\
23\end{array}$ & $\begin{array}{l}6.0 \\
4.0 \\
4.0 \\
11 \\
30 \\
28 \\
26 \\
26 \\
24 \\
26 \\
23 \\
23 \\
13 \\
33 \\
10 \\
48 \\
80 \\
48 \\
46 \\
3.0\end{array}$ & 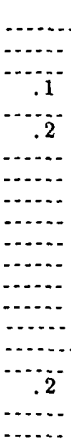 & \begin{tabular}{r}
1.2 \\
14 \\
14.4 \\
3.4 \\
4.0 \\
1.7 \\
1.5 \\
.9 \\
.2 \\
1.2 \\
3.4 \\
2.6 \\
3.0 \\
0.5 \\
\hdashline 17 \\
13 \\
9.0 \\
2.9
\end{tabular} & $\begin{array}{c} \\
.50 \\
.42 \\
.66 \\
.29 \\
.50 \\
1.2 \\
1.1 \\
0.1 \\
.06\end{array}$ & $\begin{array}{c}198 \\
288 \\
312\end{array}$ & $\begin{array}{l}182 \\
180 \\
196 \\
223 \\
238 \\
268 \\
275 \\
293 \\
248 \\
248 \\
244 \\
288 \\
252 \\
267 \\
332 \\
325 \\
376 \\
357 \\
339 \\
202\end{array}$ & $\begin{array}{r}23 \\
29 \\
29 \\
100 \\
68 \\
71 \\
66 \\
76 \\
68 \\
78 \\
78 \\
82 \\
74 \\
74 \\
82 \\
74 \\
66 \\
137 \\
131 \\
80 \\
26\end{array}$ & $\begin{array}{l}354 \\
346 \\
362 \\
465 \\
514 \\
532 \\
554 \\
576 \\
510 \\
494 \\
478 \\
571 \\
497 \\
589 \\
627 \\
739 \\
889 \\
776 \\
722 \\
377\end{array}$ & $\begin{array}{ll}7.8 & \ldots \\
7.7 & \ldots \\
8.2 & \ldots \\
7.6 & \cdots \\
7.3 & \ldots \\
8.3 & \cdots \\
7.6 & \ldots \\
7.7 & \ldots \\
7.3 & \ldots \\
7.3 & \ldots \\
7.4 & \\
7.7 & \ldots \\
7.5 & \ldots \\
7.2 & \ldots \\
7.4 & \ldots \\
7.4 & \ldots \\
7.3 & \ldots \\
7.5 & \\
7.6 & \ldots \\
7.7 & \ldots\end{array}$ \\
\hline $\begin{array}{l}206 \\
203 \\
204 \\
212 \\
207 \\
206 \\
256\end{array}$ & $\begin{array}{l}20 \\
30 \\
21 \\
24 \\
33 \\
24 \\
34\end{array}$ & $\begin{array}{r}8.0 \\
7.0 \\
6.0 \\
6.0 \\
7.2 \\
8.0 \\
30\end{array}$ & . & $\begin{array}{l}4.3 \\
2.3 \\
3.2 \\
2.8 \\
3.0 \\
2.4\end{array}$ & .10 & \begin{tabular}{r}
225 \\
\hdashline 250 \\
232 \\
249 \\
282 \\
324
\end{tabular} & $\begin{array}{l}196 \\
200 \\
202 \\
202 \\
209 \\
206 \\
250\end{array}$ & $\begin{array}{l}27 \\
34 \\
35 \\
28 \\
39 \\
37 \\
40\end{array}$ & $\begin{array}{l}376 \\
388 \\
367 \\
379 \\
401 \\
386 \\
\mathbf{5 3 6}\end{array}$ & $\begin{array}{rr}8.1 & \ldots \\
7.9 & \ldots \\
7.9 & \ldots \\
8.2 & \ldots \\
7.9 & \\
7.9 & \ldots \ldots \\
7.6 & \ldots \ldots\end{array}$ \\
\hline $\begin{array}{r}230 \\
232 \\
204 \\
255 \\
1214 \\
0222 \\
188 \\
232 \\
252 \\
242 \\
240 \\
240 \\
202 \\
222\end{array}$ & $\begin{array}{l}50 \\
44 \\
44 \\
32 \\
31 \\
27 \\
17 \\
31 \\
32 \\
30 \\
35 \\
34 \\
68 \\
11\end{array}$ & $\begin{array}{c}27 \\
30 \\
25 \\
37 \\
34 \\
30 \\
23 \\
24 \\
56 \\
34 \\
32 \\
32 \\
22 \\
3.0\end{array}$ & 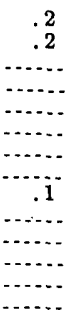 & $\begin{array}{l}3.5 \\
3.8 \\
4.3 \\
1.8 \\
3.2 \\
3.0 \\
.1 \\
3.0 \\
2.6 \\
2.8 \\
2.3 \\
2.9 \\
5.0 \\
.6\end{array}$ & 66 & \begin{tabular}{c}
317 \\
317. \\
\hdashline
\end{tabular} & $\begin{array}{l}246 \\
239 \\
228 \\
245 \\
200 \\
244 \\
177 \\
230 \\
268 \\
242 \\
246 \\
246 \\
254 \\
193\end{array}$ & $\begin{array}{l}58 \\
48 \\
61 \\
36 \\
18 \\
46 \\
23 \\
40 \\
61 \\
44 \\
50 \\
50 \\
88 \\
11\end{array}$ & $\begin{array}{l}634 \\
534 \\
488 \\
570 \\
508 \\
501 \\
391 \\
494 \\
617 \\
538 \\
531 \\
522 \\
522 \\
354\end{array}$ & 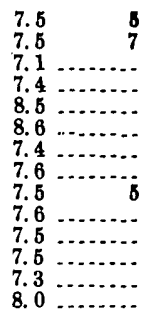 \\
\hline $\begin{array}{r}188 \\
168 \\
+204 \\
218\end{array}$ & $\begin{array}{l}20 \\
11 \\
11 \\
17\end{array}$ & $\begin{array}{l}\text { 6. } 0 \\
6.0 \\
4.0 \\
6.0\end{array}$ & $\begin{array}{c}.1 \\
\ldots . . \\
\ldots .\end{array}$ & $\begin{array}{l}1.3 \\
.6 \\
.8 \\
.5\end{array}$ & .08 & $\begin{array}{c}195 \\
210 \\
204\end{array}$ & $\begin{array}{l}178 \\
157 \\
188 \\
196\end{array}$ & $\begin{array}{l}24 \\
20 \\
18 \\
18\end{array}$ & $\begin{array}{l}339 \\
297 \\
342 \\
378\end{array}$ & $\begin{array}{l}7.8 \\
7.6\end{array}$ \\
\hline $\begin{array}{l}314 \\
222\end{array}$ & $\begin{array}{l}38 \\
24\end{array}$ & $\begin{array}{l}52 \\
4.0\end{array}$ & . & $\begin{array}{l}8.3 \\
2.8\end{array}$ & $\begin{array}{l}.14 \\
.08\end{array}$ & $\begin{array}{l}410 \ldots \ldots \\
236\end{array}$ & $\begin{array}{l}316 \\
212\end{array}$ & $\begin{array}{l}58 \\
30\end{array}$ & $\begin{array}{l}697 \\
397\end{array}$ & $\begin{array}{ll}7.9 & \ldots \\
7.7 & \ldots\end{array}$ \\
\hline
\end{tabular}


TABLE 11.-Chemical quality of

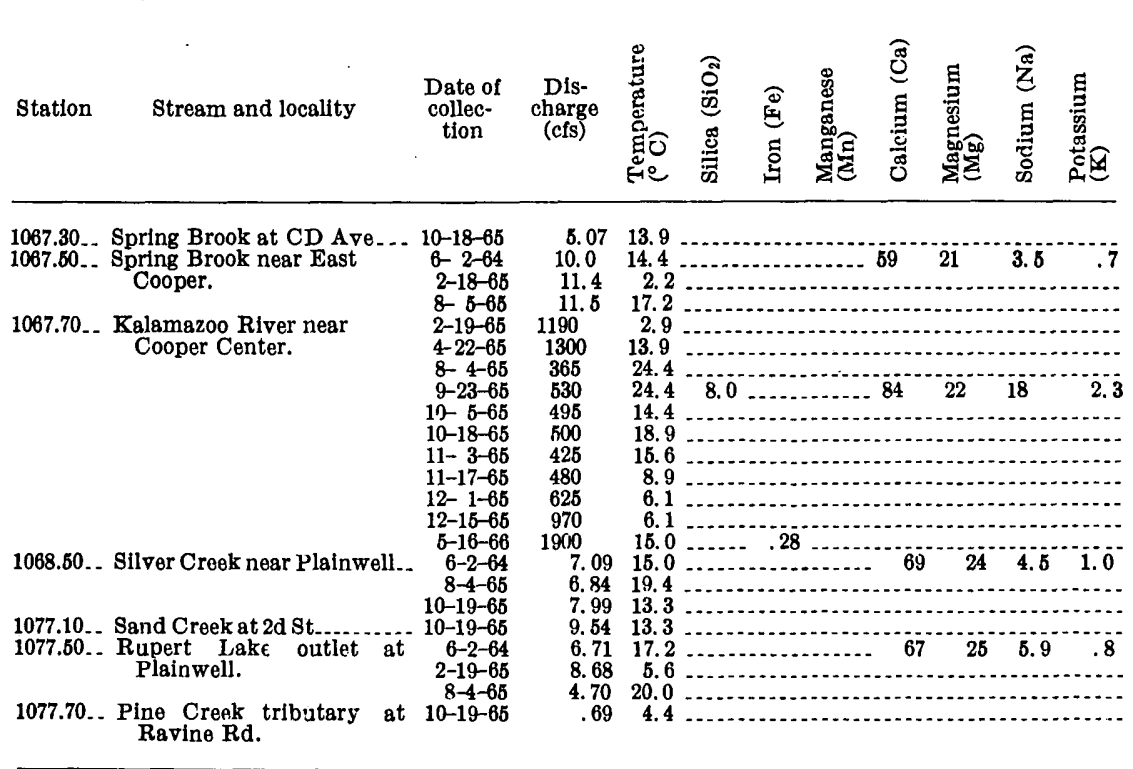

1 Also contains $4 \mathrm{mg} / \mathrm{COO}_{3}$.

Also contains $2 \mathrm{mg} / \mathrm{CO}_{3}$.

Daily mean discharge.

on Portage Creek is available to supplement the low flows. Whereas the withdrawal of Portage Creek water is large, other streams having similar potential are relatively untapped. The most significant of these are Augusta Creek and Portage River. Present withdrawal from these streams is negligible, yet they have dependable supplies of 11.0 and $12.9 \mathrm{mgd}$, respectively. Although these flows are small in comparison to Kalamazoo River, they do maintain flows through dry periods in quantities sufficient to attract industry. Limiting factors on withdrawal probably would be controlled by temperature and pollution rather than by flow rates.

\section{LAKES}

The recent expansion of water-based recreation has made lakes one of the most valuable of the county's water resources, both esthetically and as a tax base. Because of their visibility, and because they are a family-use resource, there is much public interest in their conservation.

Fluctuations in water level in lakes is a major concern of property owners or lake users, for these fluctuations affect the use and value of the property. Chemical quality of the water is also important because algae, weeds, dead fish, and floating aquatic vegetation affect the use and esthetic values of the lake. 


\begin{tabular}{|c|c|c|c|c|c|c|c|c|c|c|c|c|}
\hline \multirow{2}{*}{ 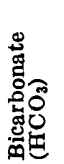 } & \multirow{2}{*}{ 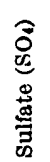 } & \multirow{2}{*}{ 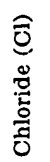 } & \multirow{2}{*}{ 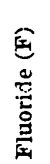 } & \multirow{2}{*}{ 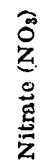 } & \multirow{2}{*}{ 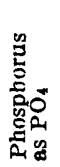 } & \multirow{2}{*}{$\begin{array}{l}\text { Dissolved } \\
\text { solids } \\
\text { (residue } \\
\text { on evap- } \\
\text { oration at } \\
180^{\circ} \mathrm{C} \text { ) }\end{array}$} & \multirow{2}{*}{$\begin{array}{c}\text { Loss } \\
\text { on } \\
\text { lgni- } \\
\text { tion }\end{array}$} & \multicolumn{2}{|c|}{$\begin{array}{c}\text { Hardness } \\
{\text { as } \mathrm{CaCO}_{3}}^{2}\end{array}$} & \multirow{2}{*}{$\begin{array}{l}\text { Specific } \\
\text { conduct- } \\
\text { ance (ml- } \\
\text { cromhos } \\
\text { at } 25^{\circ} \mathrm{C} \text { ) }\end{array}$} & \multirow[b]{2}{*}{$\mathrm{pH}$} & \multirow{2}{*}{$\begin{array}{c}\text { Color } \\
\text { (Pt-Co } \\
\text { scale) }\end{array}$} \\
\hline & & & & & & & & $\begin{array}{c}\text { Calcium, } \\
\text { mag- } \\
\text { nesium }\end{array}$ & $\begin{array}{c}\text { Non- } \\
\text { carbon- } \\
\text { ate }\end{array}$ & & & \\
\hline
\end{tabular}

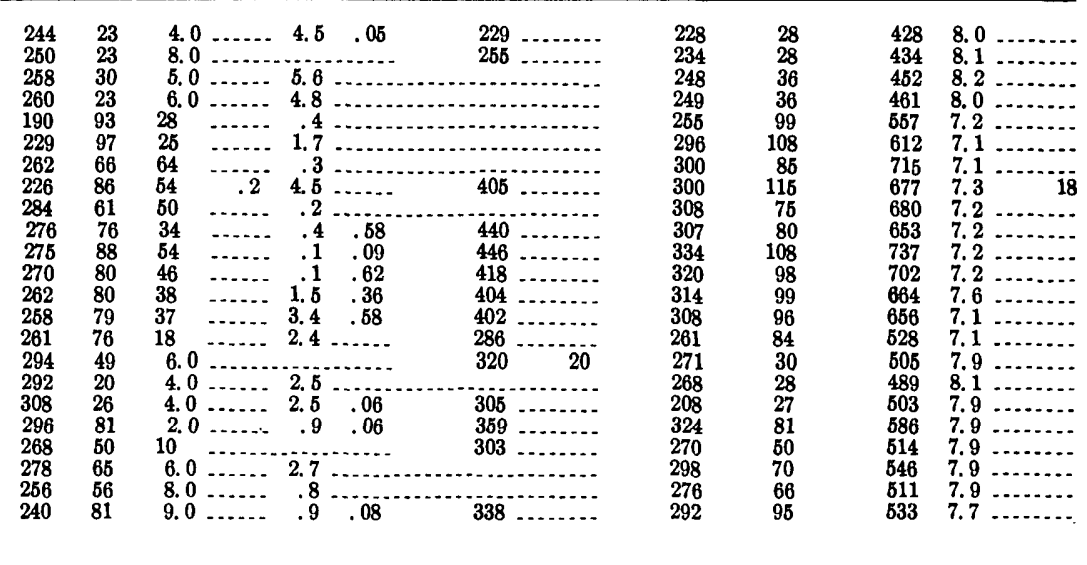

4 Also contains $8 \mathrm{mg} / \mathrm{l} \mathrm{CO}$.

Also contains $6 \mathrm{mg} / \mathrm{CO}$.

- Also contains $10 \mathrm{mg} / \mathrm{CO}$.

Some of the lakes in Kalamazoo County because of their glacial origin are called "pit" lakes. They were formed when large blocks of ice buried beneath deposits of sands and gravel melted, leaving depressions below the water table. The hydrologic significance of this origin is that the lakes and surrounding sand and gravel deposits are hydraulically connected. Thus, changes in lake levels generally reflect changes in ground-water levels.

\section{AUSTIN LAKE COMPLEX}

The most complex lake system in the county is in Portage Township just south of the city of Kalamazoo. This complex includes West, Austin, Long, Sugarloaf, and Gourdneck Lakes (fig. 1). All are considered to be part of the St. Joseph River basin as defined by surface divides. However, water-table contours on plate 4 show that the ground water moves both north and south from Austin Lake.

Detailed ground-water movement in the Austin Lake complex was determined for two different periods using water-level data from observation wells, lakes, and streams (pl. 7). The most critical period was in November 1964, when water levels reached record low stages. The other period was for May 1966 when water levels had recovered. In May 1966, the levels of West, Austin, and Long Lakes were at the same elevation for the first time since the Kalamazoo study began. 
The water-level contours on the two maps are quite similar, suggesting that the direction of ground-water flow is similar during both periods of drought and periods of above-average precipitation. Austin, West, and Long Lakes lie on or near ground-water divides and decline more rapidly during dry periods than a lake such as Gourdneck, which is not near divides. The deficient precipitation in 1960-64, shown in figure 2, was the major cause of the low levels of Austin, West, and Long Lakes. A comparison of the rate of recovery of lake levels is shown in figure 19. The lowest lake levels occurred in 1964, when the average yearly cumulative deficiency in precipitation was the greatest. Above-average precipitation in 1965-67 caused an upward trend in lake levels.

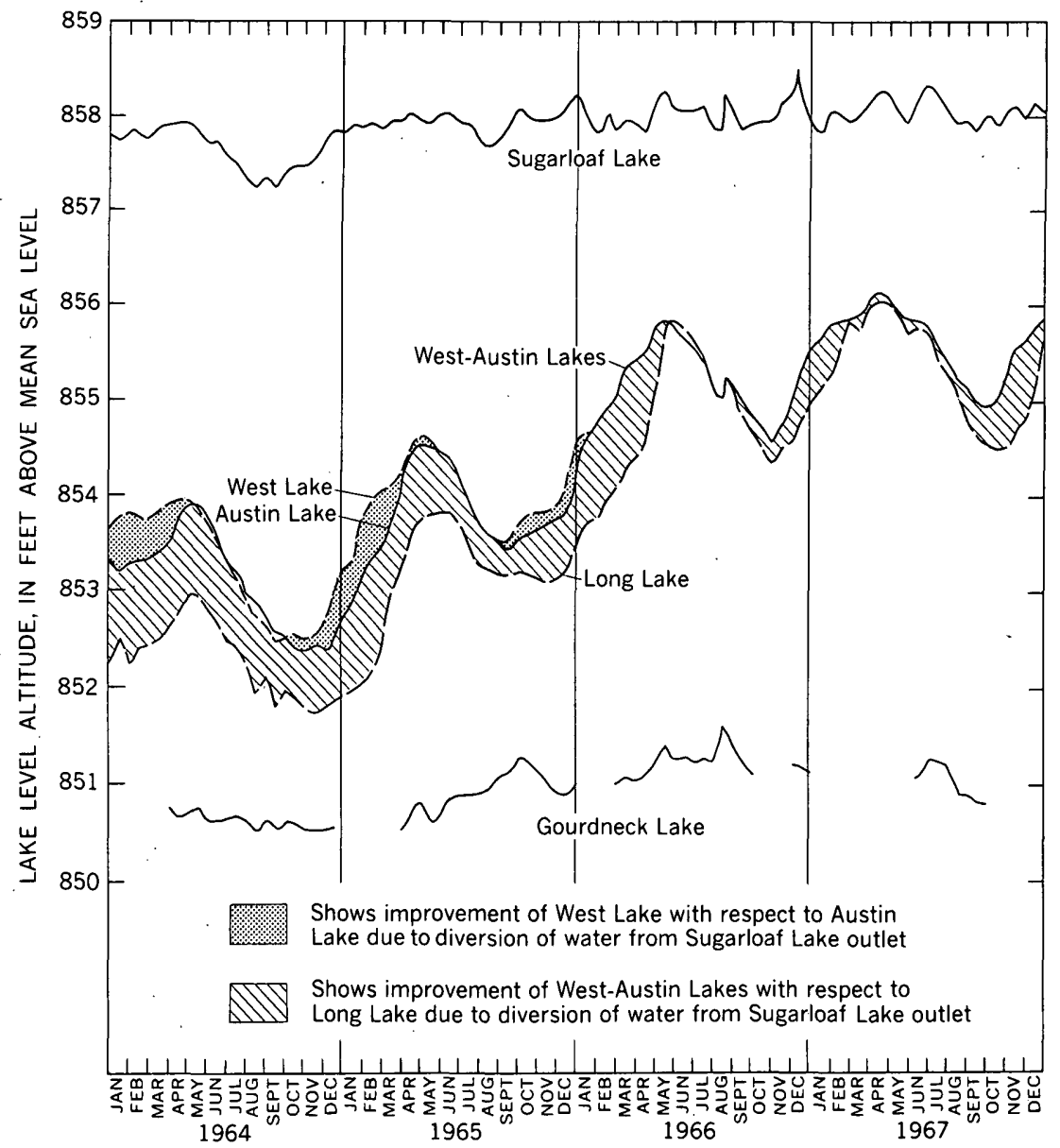

Fiaure 19.-Lake hydrographs showing improvement of water levels due to diversion of water from Sugarloaf Lake outlet to West Lake. 
In the early thirties, another period of deficient precipitation, the levels of West, Austin, and Long Lakes were low. In 1936, a diversion canal was dug to convey water from Sugarloaf Lake outlet to West Lake (pl. 7). The diversion was accomplished by building a dam on Gourdneck Creek, half a mile east of Sugarloaf Lake, and installing in the left bank at the upstream side of this dam a diversion dam to the canal. A connecting channel was also dug between West and Austin Lakes. In 1938, a channel was dug connecting Long and Austin. The water diverted to West and Austin Lakes helped to stabilize levels.

During low-water levels in 1964, the channels connecting West, Austin, and Long Lakes and the dam at the outlet channel of Austin Lake were dry. Shorelines receded and docks were far from the water's edge. Figure 20 shnws conditions during the drought.

The benefits of diverting water to West Lake during the drought period are illustrated by shaded areas in figure 19. The level of West Lake was higher than that of Austin Lake during the fall and winter months of 1964. During these months large amounts of water were diverted to West Lake. The level of this lake rose until water began to flow through the connecting channel between it and Austin Lake. The flow occurred at an altitude of about 854 feet above mean sea level but this altitude varies some because of deposition of sand in the connecting channel. Since January 1966, West and Austin Lakes have remained at the same level. The altitudes of Long Lake during 1964-67 were as much as 1 foot lower than Austin Lake and 1.7 feet lower than West Lake. From the topographic map, in 1920 the altitude of Long Lake was higher than West-Austin Lakes, so that it seems that a much greater decline would have occurred in West and Austin Lakes had no water been diverted to them during 1964 and 1965. In May 1966, all three lakes were about the same altitude and remained the same until the fall of 1966, when increased diversion to West and Austin caused their levels to rise above that of Long Lake. At altitudes higher than about 856 feet, interflow between the three lakes keeps all three lakes at about the same level.

In 1967, a new development took place in the Austin Lake complex. Water was diverted from the Upjohn Co.'s southeast recharge pond into Austin Lake (pl. 7). In December 1967, water was observed to be flowing from Austin Lake into Long Lake, yet no water was leaving Austin Lake outlet. The present altitude of the spillway dam at the outlet is 856.0 feet above mean sea level as established by Circuit Court in 1925. Probably with the diversions into West and Austin, lake levels in all three lakes can be maintained at an altitude of about 856.0 feet. 


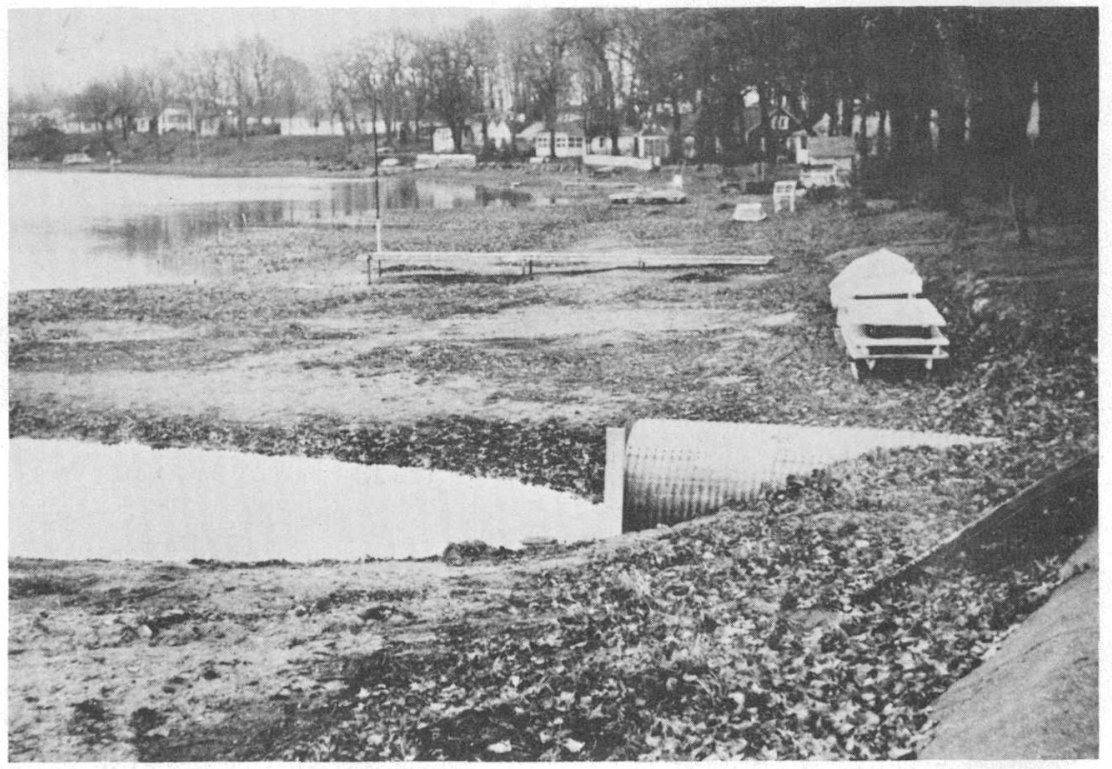

$A$

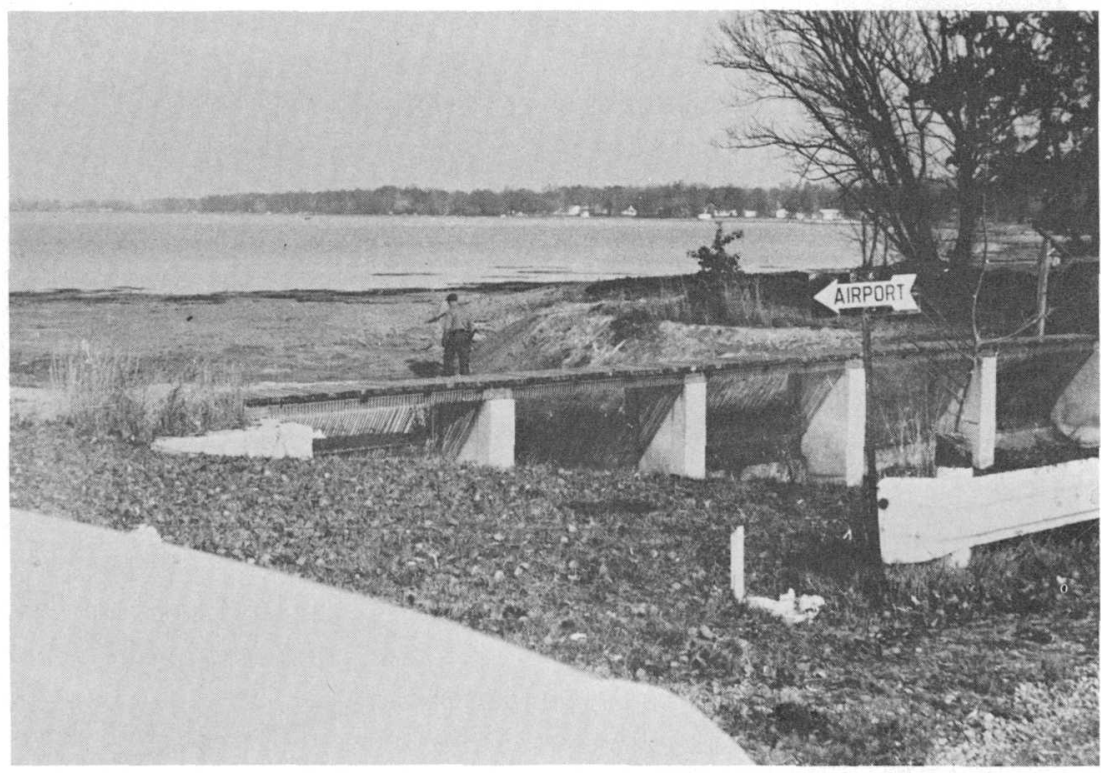

$B$

Figure 20.-Effects of drought in 1963 and 1964. A, West Lake at diversion canal inlet. $B$, Austin Lake at outlet dam. $C$, Long Lake at southwest end of lake. 


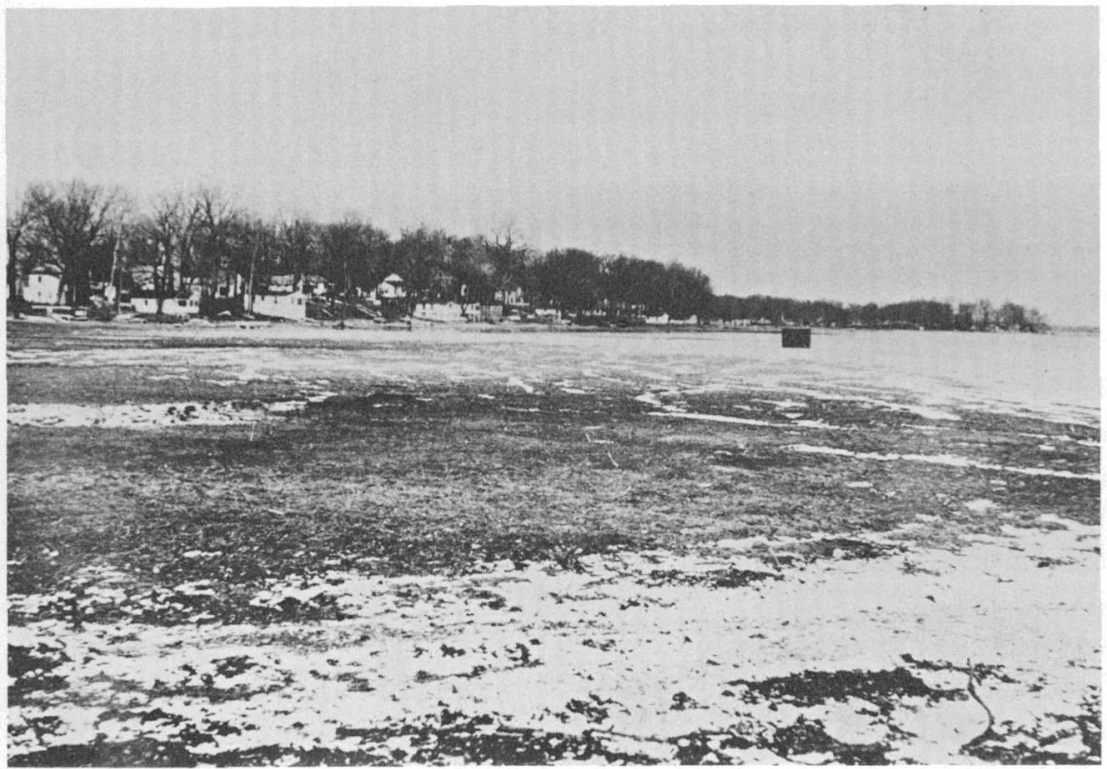

$C$

What happens to water as it is diverted from Sugarloaf Lake outlet to West Lake? How much water actually reaches West Lake and how much is lost to ground-water seepage and evaporation? These questions will be discussed in the following section.

\section{WATER BUDGET IN THE AUSTIN LAKE COMPLEX}

Most of the diversion to West-Austin Lakes takes place during the fall and winter months; therefore, the period October 1, 1965, to March 30, 1966, was selected for a water-budget study. For such a study, water gain is balanced by water loss, plus or minus changes in lake storage. Water gain by the lakes is from precipitation directly on the lakes' surface, from the diversion canal, and from subsurface inflow; water loss is from subsurface outflow and from evaporation. Little or no transpiration occurs during the fall and winter.

During the study period there was a rise of 1.9 feet in the level of Austin and West Lakes. Assuming a constant area of 1,400 acres for the two lakes, the increase in storage was 2,660 acre-ft. Precipitation at Kalamazoo State Hospital was 15.1 inches or 1,760 acre-ft. Diversion from Sugarloaf Lake outlet, measured at the gaging station on the canal was 1,370 cfs-days or 2,740 acre-ft. Subsurface inflow was estimated by the use of flow-net analysis and the modified Darcy equation 


$$
Q=T I L
$$

where $Q=$ the quantity of subsurface flow,

$T=$ the transmissibility,

$I=$ the hydraulic gradient, and

$L=$ the length of flow section.

The inflow was at a rate of $0.13 \mathrm{mgd}$ or a total of about 73 acre-ft. Therefore, the net water gained was about 4,570 acre-ft. Subsurface outflow estimated from the modified Darcy equation was at a rate of $1.9 \mathrm{mgd}$ or a total of 1,070 acre-ft. Evaporation estimates were determined by subtracting April to September 1966 evaporation totals as recorded at South Haven from the average annual lake evaporation rate of 30 inches as determined by Veihmeyer (1964). The evaporation measured at South Haven during this period was 35.29 inches. A coefficient of 0.77 was used to convert the class-A pan evaporation to equivalent lake evaporation (Kohler and others, 1959). The resultant value is 27.2 inches. Therefore, the evaporation from the lakes during the period October to March 1966 was approximately 2.8 inches $(30.0-27.2)$ or about 326 acre-ft. Therefore, the net water loss was about 1,400 acre-ft. A comparison of the water-budget elements follows:

Water budget, Austin Lake complex, October 1, 1965-March 31, 1966

[Units are acre-feet]

\begin{tabular}{|c|c|c|c|}
\hline Budget element & $\begin{array}{l}\text { Water gained } \\
\text { (inflow) }\end{array}$ & $\begin{array}{l}\text { Water loss } \\
\text { (outflow) }\end{array}$ & $\begin{array}{c}\text { Change in } \\
\text { storage }\end{array}$ \\
\hline $\begin{array}{l}\text { Precipitation } \\
\text { Diversion canal } \\
\text { Subsurface inflow }\end{array}$ & $\begin{array}{r}1,760 \\
2,740 \\
73\end{array}$ & & \\
\hline Subsurface outflow & & 1,070 & \\
\hline Evaporation & $-1-2-1$ & 326 & \\
\hline Rise in lake level & & & 2,660 \\
\hline Total & 4,573 & 1,396 & 2,660 \\
\hline
\end{tabular}

Deficit due to estimation errors

517

The deficit in water loss can be attributed to errors in determining evaporation, subsurface inflow and outflow, or to losses through the diversion canal.

The remainder of the 1966 water year, April 1 to September 30, also was analyzed. During this period the precipitation was 19.5 inches or 2,280 acre-ft, the diversion was 934 cfs-days or 1,870 acre-ft, and the subsurface inflow was $0.26 \mathrm{mgd}$ or 146 acre-ft. Therefore, the net gain of water was about 4,300 acre-ft. As previously stated, the April to September loss to evaporation as measured at South Haven was 27.2 inches or 3,160 acre-ft. Subsurface outflow was estimated to 
be $1.8 \mathrm{mgd}$, or 1,010 acre-ft. Therefore, the net loss of water was 4,170 acre-ft. During this period there was no surface outflow from Austin Lake. Levels in the two lakes declined by 0.6 foot, or there was a loss in storage of 840 acre-ft. A comparison of the water-budget elements follows:

Water budget, Austin Lake complex, April 1, 1966-September 30, 1966

[Units are acro-feet]

\begin{tabular}{|c|c|c|c|}
\hline Budget element & $\begin{array}{l}\text { Water gained } \\
\text { (inflow) }\end{array}$ & $\begin{array}{l}\text { Water loss } \\
\text { (outflow) }\end{array}$ & $\begin{array}{l}\text { Change in } \\
\text { storage }\end{array}$ \\
\hline $\begin{array}{l}\text { Precipitation } \\
\text { Diversion canal } \\
\text { Subsurface inflow }\end{array}$ & $\begin{array}{l}2,280 \\
1,870\end{array}$ & (n) & \\
\hline $\begin{array}{l}\text { Subsurface inflow } \\
\text { Subsurface outflow }\end{array}$ & 146 & 1,010 & \\
\hline Evaporation & $\ldots$ & 3,160 & \\
\hline & & & I \\
\hline Total ... & 4,296 & 4,170 & 84 \\
\hline
\end{tabular}

The deficit in water loss can be attributed to transpiration and errors in determining evaporation, subsurface inflow and outflow, and losses through the diversion canal.

The winter and spring months are the best times for diverting water to West Lake, because streamflow is generally higher and the demands of water users are not as great as in the summer and fall months. However, more diversion during summer months would maintain lake levels during periods of major recreational use. In the reach of the diversion canal between the staff gage at the diversion dam and the gaging station (pl. 7), much of the water lost to ground-water seepage returns to Gourdneck Creek. Below the gaging station, loss is to Portage Creek. A reconnaissance of Gourdneck Creek between the gaging station and Gourdneck Lake revealed a notable increase in flow. Water-level contours shown on plate 7 substantiate the direction of ground-water flow. One can conclude that diversion of water from Sugarloaf Lake outlet not only benefits West-Austin Lake levels but recharges the aquifer which in turn discharges into Gourdneck Lake, Gourdneck Creek, and Portage Creek. When water levels of WestAustin Lakes are at altitudes above 856.0 feet, outflow from Austin Lake returns to Gourdneck Creek via the Austin Lake outlet.

In summary, it appears advantageous to utilize the large supply of water from Sugarloaf Lake to maintain West-Austin Lake levels. The diversion canal acts as a conveyor of water not only to West-Austin Lakes but also as a means of recharging water to the creeks and the ground-water aquifers. Increasing the diversion during the summer 
months would maintain satisfactory levels on West and Austin Lakes with relatively minor effects on the levels of Gourdneck Lake and Sunset Lake.

Sugarloaf Lake outlet, the source of diversion water, is located in Portage and Schoolcraft Townships. The control structure at the head of the diversion canal is operated by the Simpson-Lee Paper Co. located in Vicksburg. Water in excess of the company's needs is diverted to West-Austin. During the period June to September 1964, an estimated average flow of $190,000 \mathrm{gpd}$ was diverted to West-Austin Lakes. During water years 1965-67, average amounts diverted were $2.37,4.06$, and $4.06 \mathrm{mgd}$, respectively. Most of this diversion, however, took place during the periods December through May. Data on runoff in the canal are presented below :

Summary of diversion flow to West-Austin Lakes

[Gourdneck Canal near Schoolcraft]

\begin{tabular}{|c|c|c|c|c|}
\hline \multirow{2}{*}{ Water year } & \multirow{2}{*}{$\begin{array}{l}\text { Total (cfs- } \\
\text { days) }\end{array}$} & \multirow{2}{*}{$\begin{array}{c}\text { Average rate } \\
(\mathrm{mgd})\end{array}$} & \multicolumn{2}{|c|}{$\begin{array}{l}\text { Total during December to } \\
\text { May }\end{array}$} \\
\hline & & & Cfs-days & Percent \\
\hline $\begin{array}{l}1965 \\
1966 \\
1967\end{array}$ & $\begin{array}{l}1,337 \\
2,301 \\
2,299\end{array}$ & $\begin{array}{l}\text { 2. } 37 \\
\text { 4. } 06 \\
\text { 4. } 06\end{array}$ & $\begin{array}{l}1,134 \\
1,557 \\
1,964\end{array}$ & $\begin{array}{l}\text { 84. } 8 \\
67.7 \\
85.4\end{array}$ \\
\hline
\end{tabular}

Diversion during the months of June through September is small, not enough to sustain satisfactory levels on West-Austin Lakes. Figure 19 shows that the levels of Sugarloaf and Gourdneck Lakes are relatively steady, and although increasing the diversion to WestAustin Lakes during the summer months may have some effect on Gourdneck Lake, ground-water inflow to the lake is ample to maintain satisfactory levels. Should the level of Gourdneck Lake decline to undesirable levels, diversion could be discontinued until the lake reached a desired level.

Gourdneck Creek both above and below Gourdneck Lake meanders through marshland. The creek is shallow and the channel contains many cattails and other forms of aquatic growth. The channel widens and meanders considerably before reaching Sunset Lake. The water here is shallow with an abundance of lily pads and other aquatic growth. When water temperature increases, aquatic vegetation is more abundant, and more water is lost to evaporation and transpiration. Filling this marshland would reduce evapotranspiration losses and increase the available supply of water to Gourdneck and Sunset Lakes. This in turn would provide more water for diversion to WestAustin Lakes. 
The effects of pumping ground water into Crooked and Eagle Lakes (fig. 1) are illustrated in figure 21. Lake gage readings were adjusted to an arbitrary datum for comparison purposes.

Prior to pumping, Crooked Lake, Eagle Lake, and adjacent Bass Lake responded similarly to climatic and seasonal changes. To evaluate the initial effects of pumping at Crooked Lake, water levels were compared to Eagle Lake. The deviation in the levels of Crooked Lake from the levels of Eagle Lake increases until an equilibrium state is reached. When this occurs, levels of Crooked Lake with respect to Eagle Lake remain relatively constant. Subtracting the water levels of Eagle Lake from Crooked Lake during April through November 1965 gives the effects of ground-water pumpage at Crooked Lake.

Bass Lake, which is lower on the ground-water gradient than Crooked Lake, benefited from the increased head created by pumping at Crooked Lake. After about 4 months of pumping at Crooked Lake, the levels of Bass Lake reached an equilibrium state with respect to Crooked and Eagle Lakes. Bass Lake was therefore used as an index to determine the effects of pumping into Eagle Lake.

Pumping was stopped at Crooked Lake in October 1966 and at Eagle Lake in January 1967. During 1967 pumping occurred intermittently at both lakes and caused the nonconformity in lake levels during the year.

About 33 percent of the water added to Crooked Lake from pumpage and precipitation was lost to transpiration and ground-water seepage during the period April 10 to September 30, 1965. The increase in stage from April 10 (when pumping began) to September 30 was 1.06 feet. Evaporation losses during this period, based on U.S. Weather Bureau class-A evaporation pan records at South Haven, adjusted by a coefficient of 0.77 (p. 59), amounted to 26.5 inches, or about 350 acre-ft. The total available supply to the lake from pumping and precipitation was about 780 acre-ft. Reducing this supply by the evaporation losses and storage leaves about 260 acre-ft lost to groundwater seepage and to transpiration by vegetation.

Much of the apparent loss returns to the water table and is recycled through the system by pumpage. Two shallow observation wells located 25 feet from the pumped well indicate that the drawdown caused by pumping extended under Crooked Lake. The infiltration of water into the area of influence of the pumped well resulted in recycling of part of the water pumped from the well. Data available are not sufficient to determine the amount of water recycled.

Lakes which are separated from deep aquifers by a thick aquiclude have the greatest potential for improvement in their levels by ground- 


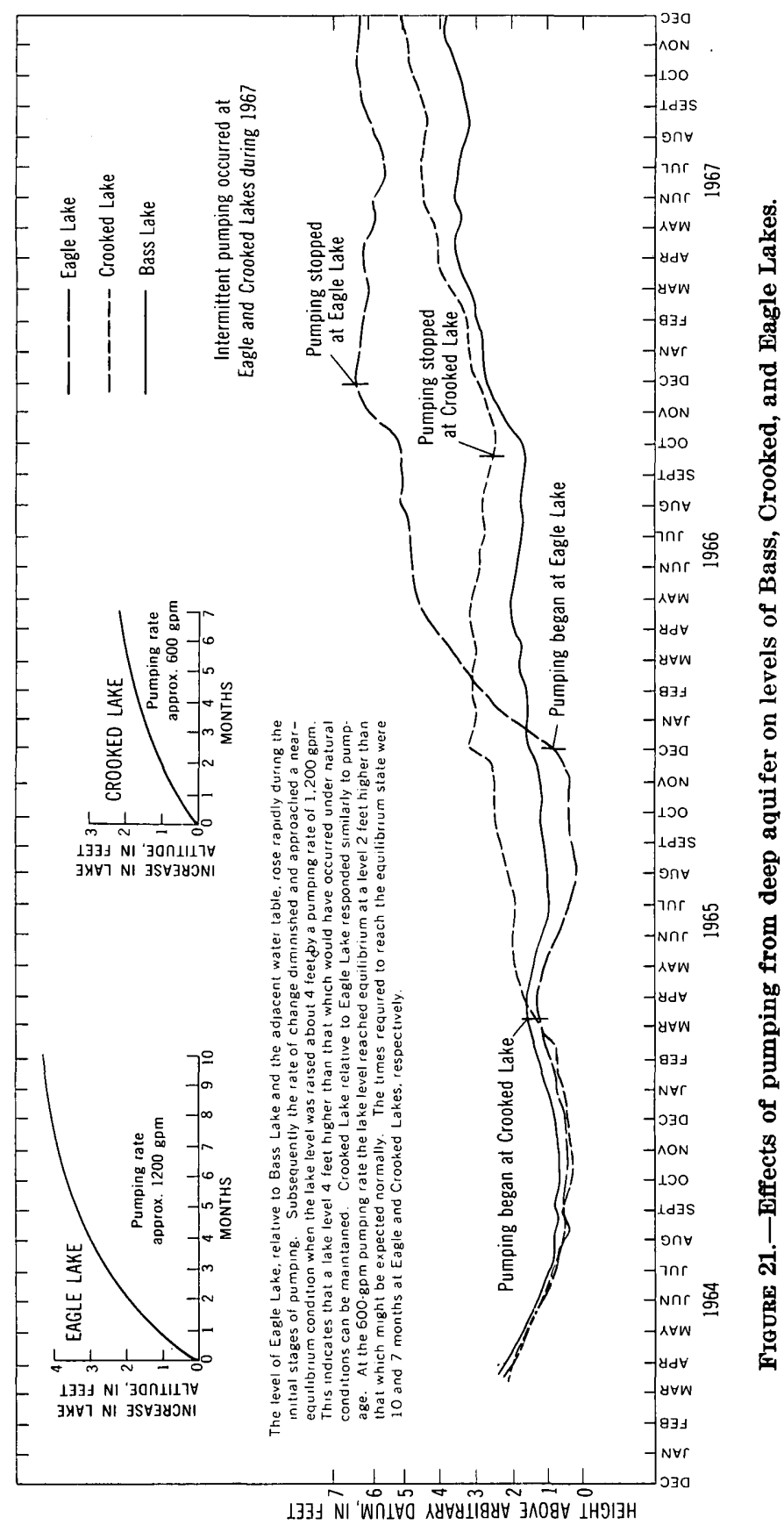


water augmentation. The lower aquifer in Kalamazoo County is shown on plate 1 . In some areas, the effects of pumping water from a deep aquifer into a lake may have an adverse effect on the existing development of the aquifer. This should be a primary consideration. given to any proposed pumping.

The use of a well to raise lake levels can be made very effective by coordinating the period of pumping with the seasonal use. Most lakes are used for recreation and receive their most intense use during the summer months; therefore, water-level augmentation by pumping should be effective for this period.

Curves constructed from data collected on Crooked and Eagle Lakes (fig. 21) show the relationship between time and the relative improvement in the level of these lakes. Crooked Lake, for example, requires about 7 months of continuous pumping at $600 \mathrm{gpm}$ to raise the level of the lake 2 feet above the level it would have, had there been no pumping. The rate of change of potential lake level decreases with time; in fact, nearly one-half of the altitude rise is gained in the first 2 months. It should be realized that figure 21 represents the rise above the unaugmented lake level. For example, the actual lake levels may decline during the summer when evapotranspiration is greater than the pumping rate, but they decline at a much reduced rate.

Figure 22 illustrates, for Crooked and Eagle Lakes, the effects of. adding water to control the altitude of the lakes. For illustration
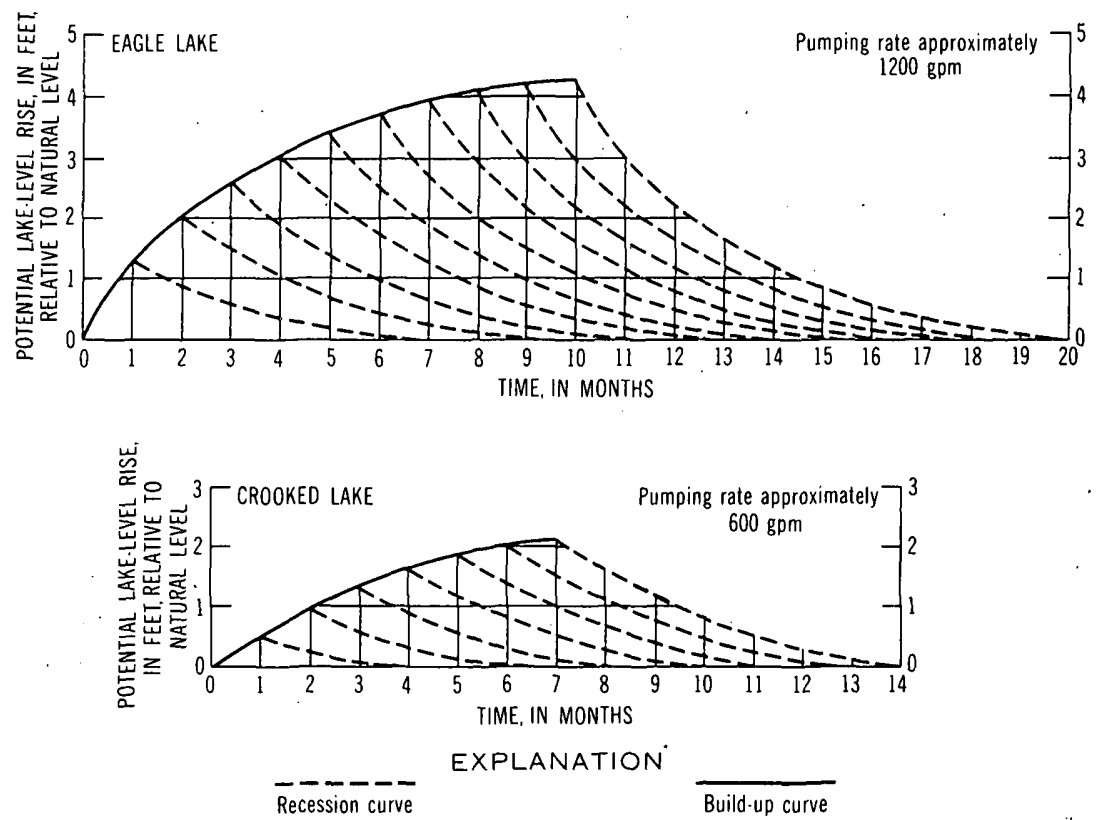

FrgURE 22.-Relationship between lake-level improvement and period of pumping. 
purposes, assume the maximum lake level is desired in August. To accomplish this, pumping should begin in February at Crooked Lake and in November at Eagle Lake. The decline of lake levels after pumping stops, relative to natural conditions, will follow the dashed curves. These curves were developed on the basis of the image-well theory, whereby the basic curve of rise is the mirror image of the basic curve of decline.

The cost of operation may make it undesirable to pump water continuously into Crooked and Eagle Lakes. A method of intermittent pumping can be used to give a smaller rise at less cost. Figure 23 illustrates the potential lake-level rise at Eagle Lake associated with pumping at alternate months. The maximum rise is one-half of that obtained by continuous pumping.

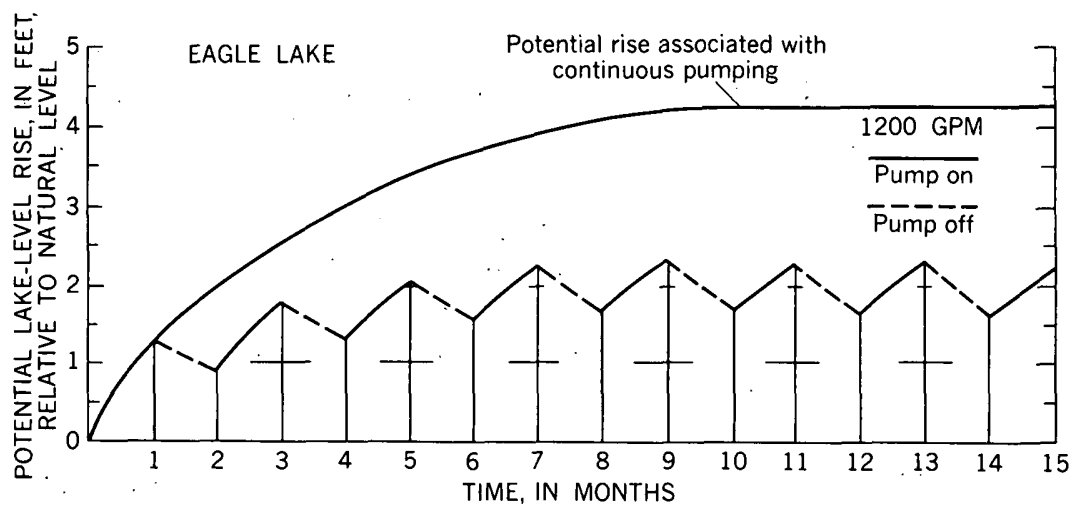

Froure 23.-Effects of adding ground water to Eagle Lake on alternate months.

\section{LAKES AND RESERVOIRS AS A SOURCE OF WATER SUPPLY}

Lake levels in Kalamazoo County are summarized in table 12. Most of the records are of short duration, having been collected only during this project. Lakes are natural impoundments of surface water and may be considered as sources of water supply. The water-surface area of the lakes is approximately 11,700 acres (Humphrys and Green, $1962)$ or about 3 percent of the total area of the county. An average fluctuation of 2 feet in lake levels over this area is equivalent to a volume of 23,400 acre-ft or a runoff of 0.75 inch from the county's $572 \mathrm{sq} \mathrm{mi}$ of area-probably a conservative estimate of the usable natural storage in these lakes. However, the use of lakes as sources of water supply may decrease their recreational value. Decisions as to most desirable use must be based on consideration of these conflicting interests.

Manmade storage reservoirs, like many natural lakes, have multipleuse features in that they may be used for recreational purposes (boat- 


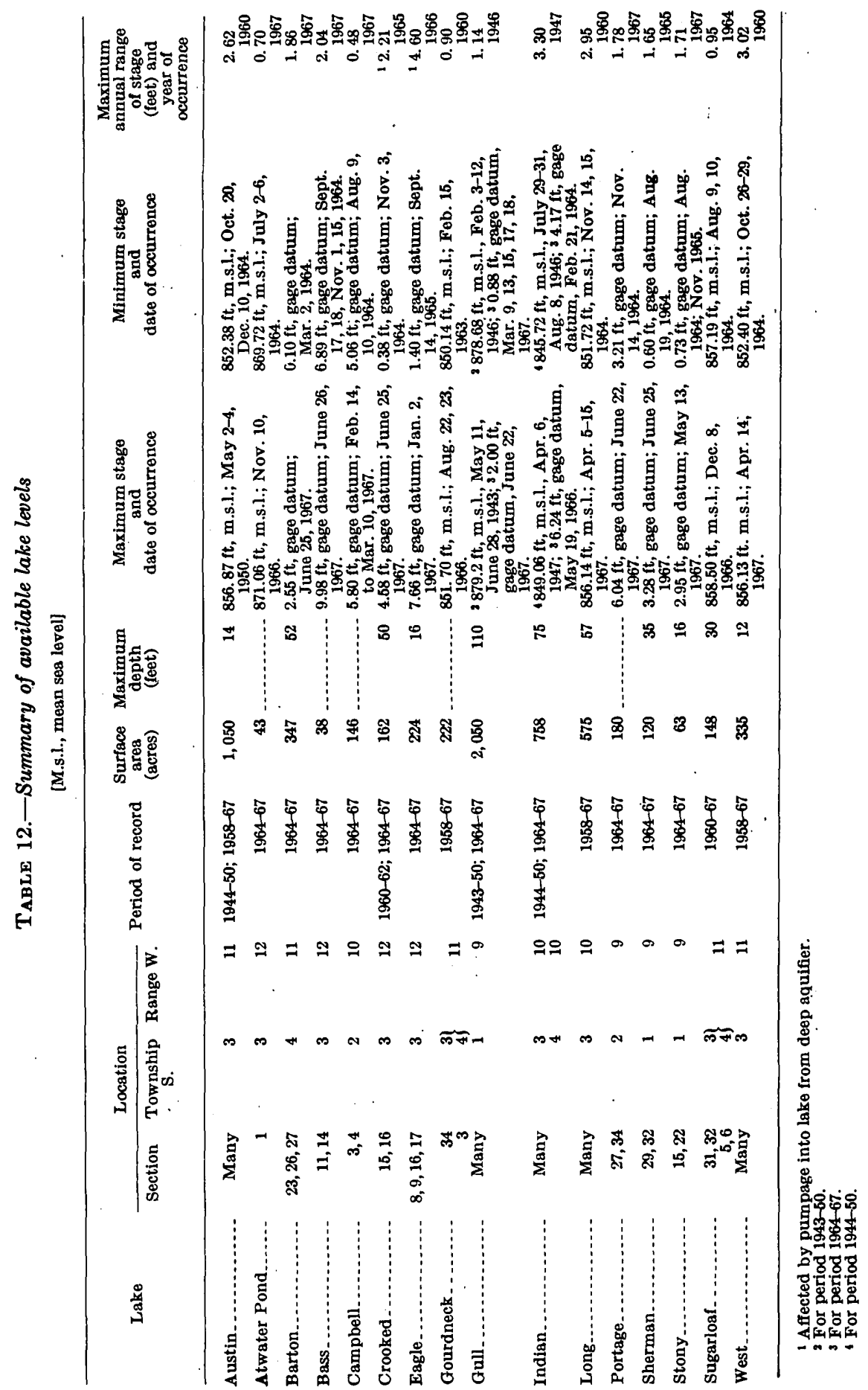


ing, fishing, swimming), esthetic value (parks, picnic areas), recharge areas to ground-water aquifers, industrial supply (multiple uses), flood control, water supply, and augmentation of streamflow during periods of low flow. These uses are often considered in justifying the cost of storage reservoirs. From a map reconnaissance, 20 locations were selected as possible damsites, data for which are contained in table 13. The locations of most of these sites are shown in figure 24 . Economic and hydrologic factors may rule out the feasibility of some of these reservoirs. The data shown in table 13 are approximate and useful only for preliminary planning. Further detailed field investiga-

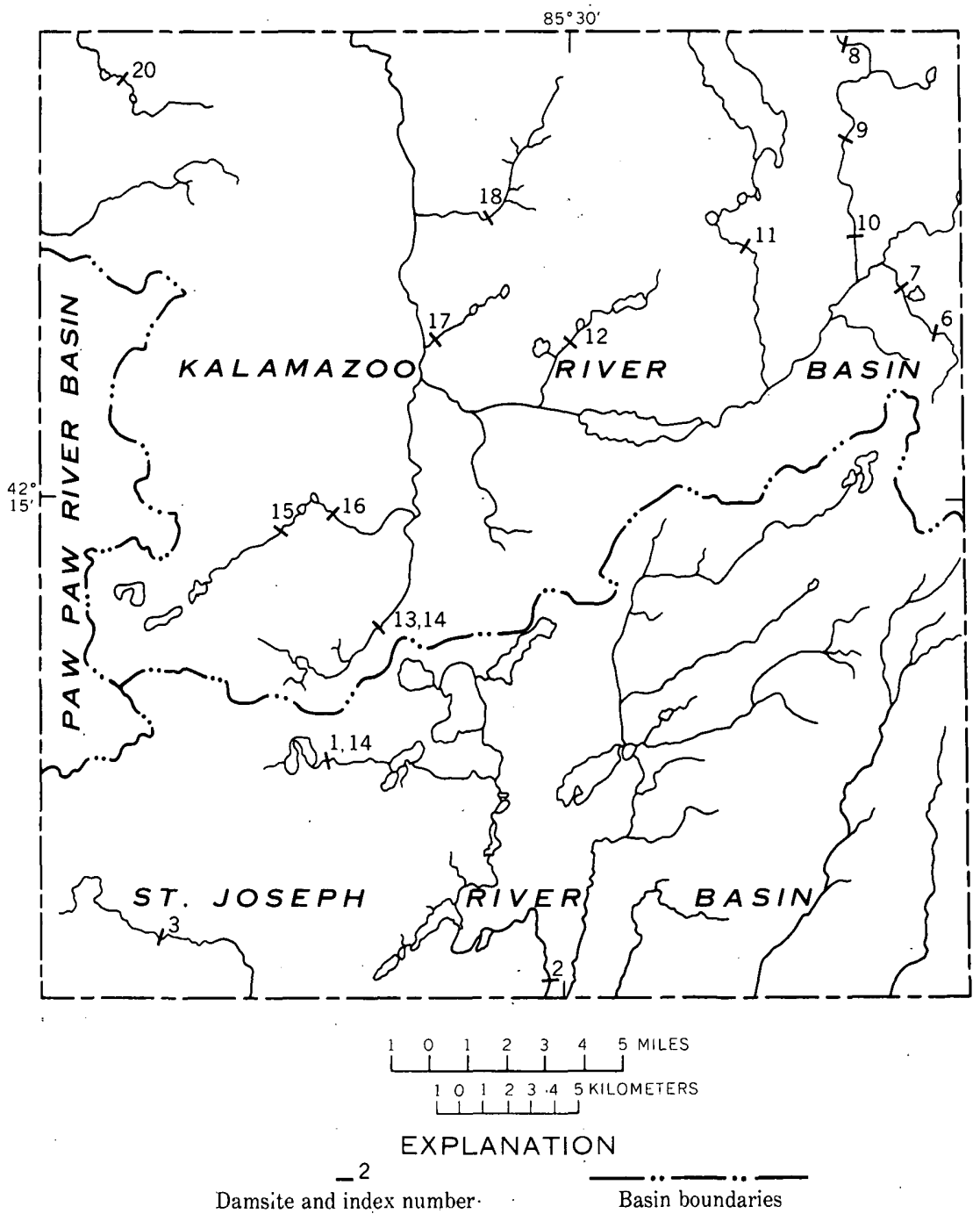

Froure 24.-Locations of possible damsites. 


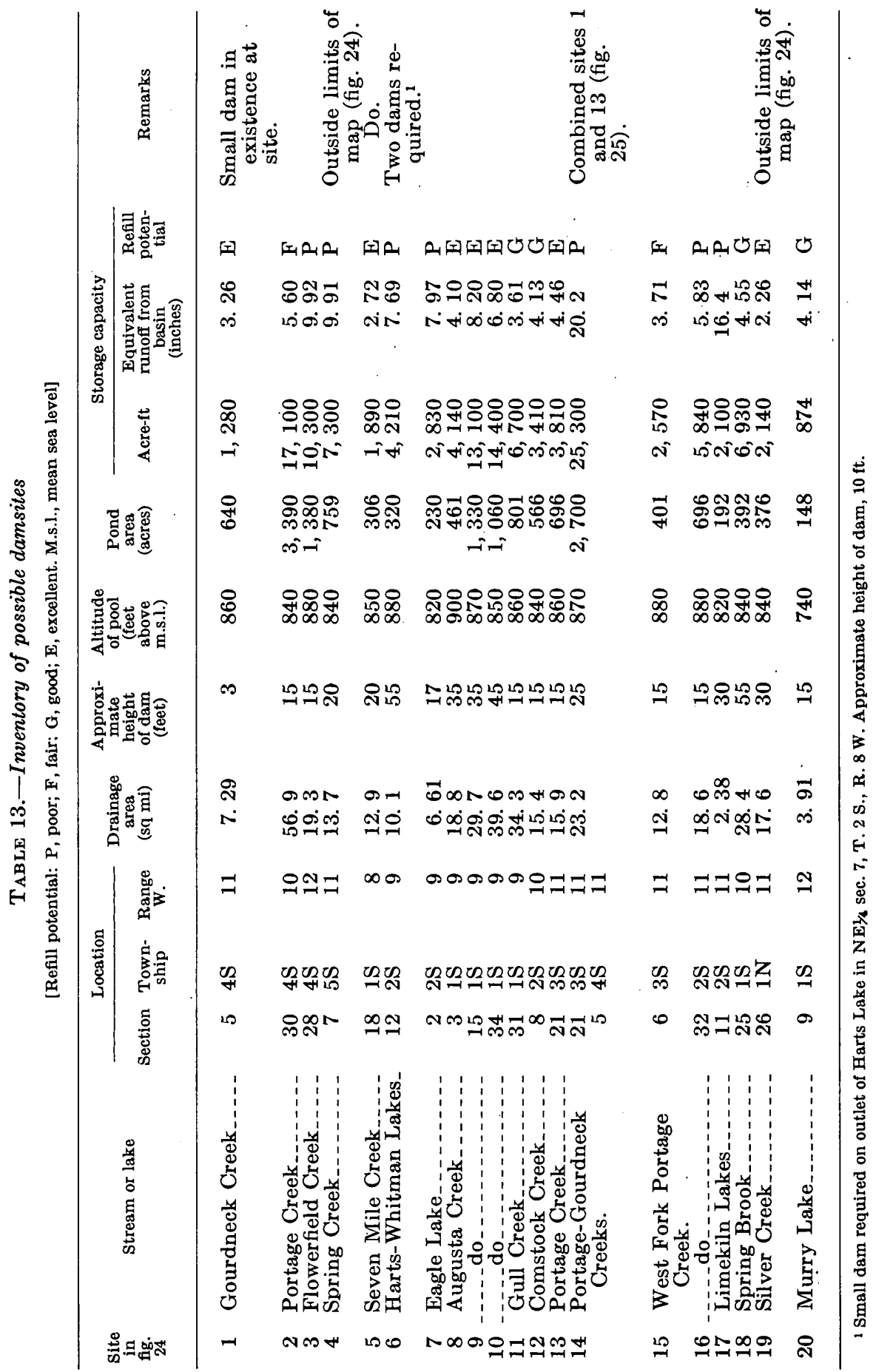


tions and the procurement of more detailed topographic maps would be needed for any project planning or design.

The refill potential indicated in table 13 was rated on the probability of replacing the indicated storage volume annually. No adjustment was made for increased evaporation loss. A conservative estimate of evaporation loss from a reservoir would be 2 feet per year. Storage capacities for some reservoirs are large considering the size of the drainage basin. To fill these reservoirs in 1 year may be detrimental to users of water downstream. Therefore, storage for periods longer than 1 year would probably be required to fill the reservoirs. Runoff figures, determined for streams having continuous streamflow records, were used to rate the refill potential of reservoirs located on these streams. For other sites, the refill potential was rated excellent if the storage capacity represented less than 3.5 inches of runoff from its drainage basin, good if between 3.5 and 4.5 , fair if between 4.5 and 6.0, and poor if greater than 6 inches. For example, the reservoir on Seven Mile Creek would require a storage equivalent of 2.72 inches of runoff. Available streamflow records in the area indicate that at least this volume of runoff can be expected every spring. The refill potential can, therefore, be rated excellent.

There are no potential reservoir sites on the Kalamazoo River within the county. The development of reservoirs on any of the tributaries of the Kalamazoo River would not be beneficial in augmenting low flow in the Kalamazoo River, nor would these reservoirs contain sufficient quantities of water for large municipal uses.

There are several potential damsites located in the Augusta Creek basin. The annual runoff from this basin is higher than from most basins in the county, and the basin maintains relatively high flows even during dry periods. The topography is favorable for reservoir development and the impoundment areas are relatively undeveloped. The determination of reservoir size was somewhat arbitrary, but in general, it was designed to insure that a runoff of no more than 10 inches would be required to fill the reservoir. During water years 1965-67, runoff at the gaging station on Augusta Creek amounted to $10.58,11.23$, and about 13.0 inches, respectively. This is more than adequate to fill and maintain any one of the reservoirs as shown for Augusta Creek. Slightly larger reservoirs could probably be filled and maintained on Augusta Creek. However, water used in the development of one reservoir on Augusta Creek would not be available for storage in other reservoirs on the creek.

Two reservoir sites, 1 and 13 (fig. 24), on Portage Creek and Gourdneck Creek may be most useful for water management. As discussed earlier, a small dam exists on Gourdneck Creek and is used 
to regulate the outflow from Sugarloaf Lake. Another excellent site in terms of refill potential is on Portage Creek above the city of Portage. The headwaters of Portage and Gourdneck Creeks are adjacent to one another and are separated by the topographic divide of the St. Joseph and Kalamazoo River basins. If two dams were constructed, one on Portage Creek and one on Gourdneck Creek, both with a crest altitude of 870 feet above mean sea level, they would provide a single reservoir with 25,000 acre-ft of storage (fig. 25). The combination of these sites would require 20.2 inches of runoff to fill the reservoir. Runoff figures for the gaging station on Portage Creek near Portage for water years 1965-67 were 13.82, 15.83, and 19.3 inches, respectively, and for the gaging station on Gourdneck Creek, 11.0, 17.07, and 20.95 inches. The runoff would not be sufficient to fill a reservoir of this size in 1 year, but the reservoir could be filled with overyear storage. Necessary release to downstream users might delay the filling of the reservoir over a period of several years. The important issue here is not the time required to fill the reservoir but the benefits that could be derived. Storage of 25,000 acre-ft of water in the headwaters of Portage Creek and Gourdneck Creek, with facilities to divert this water to the various users in both basins, would provide a useful management tool in this hydrologically complex region. The area that would be inundated presently (1968) is not highly developed. A study of land values and other economic, legal, and esthetic considerations would be needed to determine the feasibility of this development.

Another consideration is the effect a reservoir might have on ground-water runoff. An impoundment at or near a ground-water divide may cause diversion of ground-water runoff so that the same amount of water is no longer available at the higher discharge level. However, it is believed that if such diversion of ground-water runoff occurred because of the Portage-Gourdneck reservoir, the water diverted would still be retained in the Portage and Gourdneck Creek basins.

\section{CHEMICAL QUALITY}

The natural process of lake filling with sediment is accelerated by man's urbanization and agricultural practices. The increased load of inorganic sediments results primarily from construction activities and farming. In addition to this influx of inorganic sediment, the lakes may fill with organic sediments which result from aquatic plants which grow, die, and accumulate on the bottom of the lakes. This natural organic filling process is accelerated by the introduction of nutrients, particularly nitrate $\left(\mathrm{NO}_{3}{ }^{-}\right)$and phosphate $\left(\mathrm{PO}_{4}^{-3}\right)$. These nutrients are derived from septic-tank effluent, agricultural fertilizer, barnyard drainage, or any other sources rich in nitrate and phosphate. 


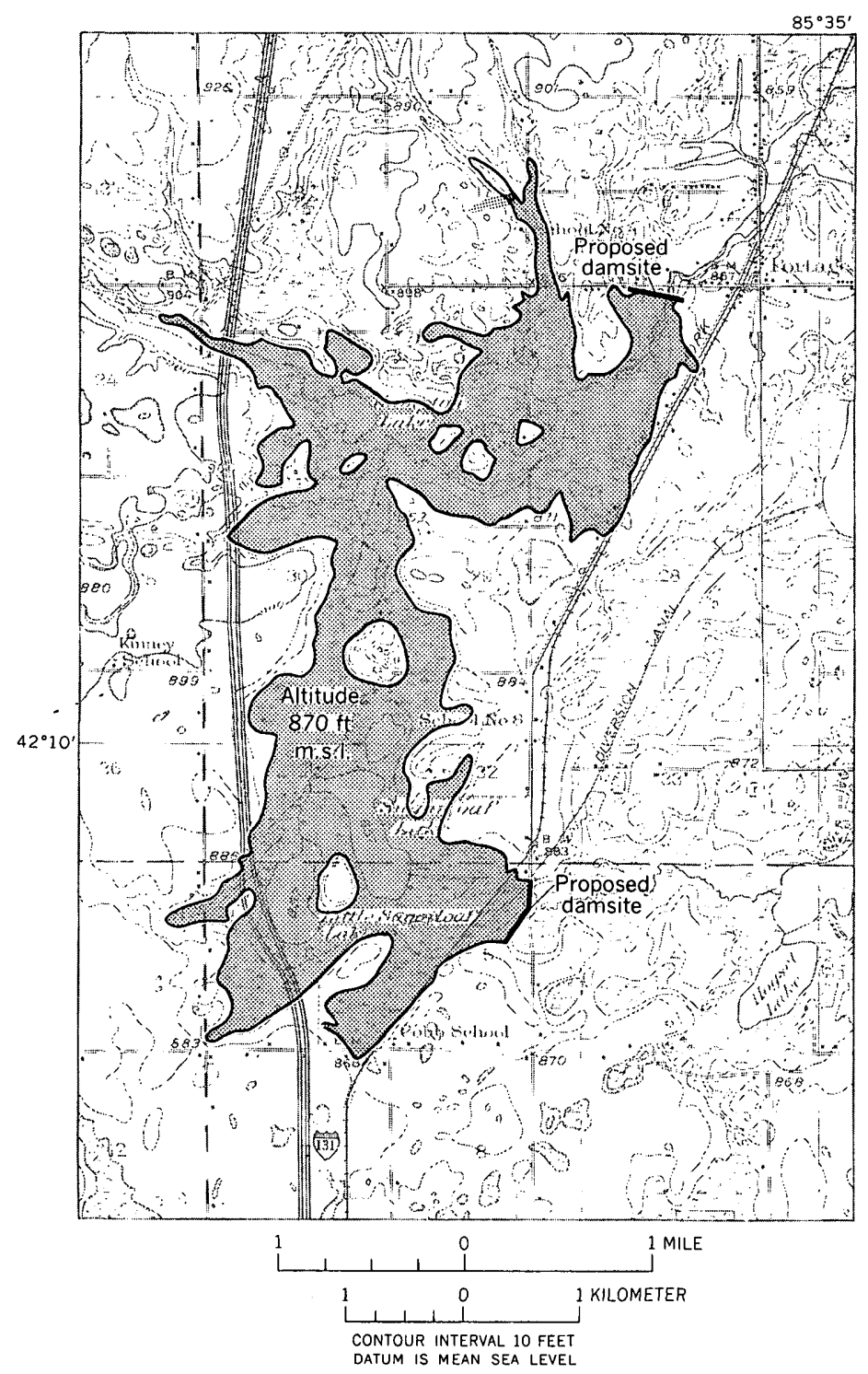

Figure 25.-Area that would be inundated by Portage-Gourdneck Creeks reservoir.

Once these nutrients have been introduced into a lake, plant growth is initiated that is difficult to retard by merely reducing nutrient inflow. The sequence starts with accelerated growth of aquatic vegetation, which dies and settles to the bottom. This vegetation decays, releasing nutrients for more plant growth. The cycle thereby perpetuates itself. Because the hydrology and ecology of individual lakes 
differ, adding a certain amount of nutrient per volume of water would not have the same effect on all lakes. However, it is desirable to keep nutrient-rich effluent out of lakes to avoid accelerated weed growth and lake filling.

\section{AUSTIN AND WEST LAKES}

The quality of water in Austin and West Lakes is generally good and is about the same in each lake (table 14). Analyses of samples taken on the same day reveal that Austin Lake has lightly higher concentrations of chemical constituents except dissolved oxygen, perhaps indicating more vegetation growth in West Lake. Seasonal variations in quality, however, are greater than are the differences in quality between lakes. Future recreational uses of these shallow lakes depend largely upon the quality and quantity of incoming water from Sugarloaf Lake and the quality and temperature of water currently pumped into Austin Lake from the Upjohn Co.'s southeast recharge pond. If the nutrient content remains low and other contaminants are controlled, these lakes will be suitable for recreational purposes for many years.

The relatively low concentration of dissolved solids of these lakes with respect to ground water and their elevation with respect to the water table (pl. 7) indicate that they serve as areas of recharge to the ground-water reservoir. The benefit of this relationship is that local septic-tank effluent probably does not drain into the lake.

The greater the flow into West and Austin Lakes, the greater the flushing action which tends to remove some nutrient-rich water. This assumes that the water flowing into the lake will retain its present high quality.

\section{LONG LAKE}

Long Lake, which is close to West and Austin Lakes, is chemically similar to these lakes (table 14). The lake receives ground water from the southeast and discharges it to the northwest. The ground-water movement into and out of the lake in a given year is very small in relation to the total volume of the lake; therefore, it might require a rather long period of time to clean up the lake if it were allowed to become enriched in nutrients.

\section{SUGARLOAF AND GOURDNEGK LAKES}

Sugarloaf and Gourdneck Lakes are both fed by ground water and are connected by Gourdneck Creek. The lakes in this system are chemically similar to one another, having generally higher hardness and bicarbonate content than Austin, West, and Long Lakes. The similarity in quality of Sugarloaf and Gourdneck Lakes is a reflection of the quality of ground-water inflow to the lakes. 


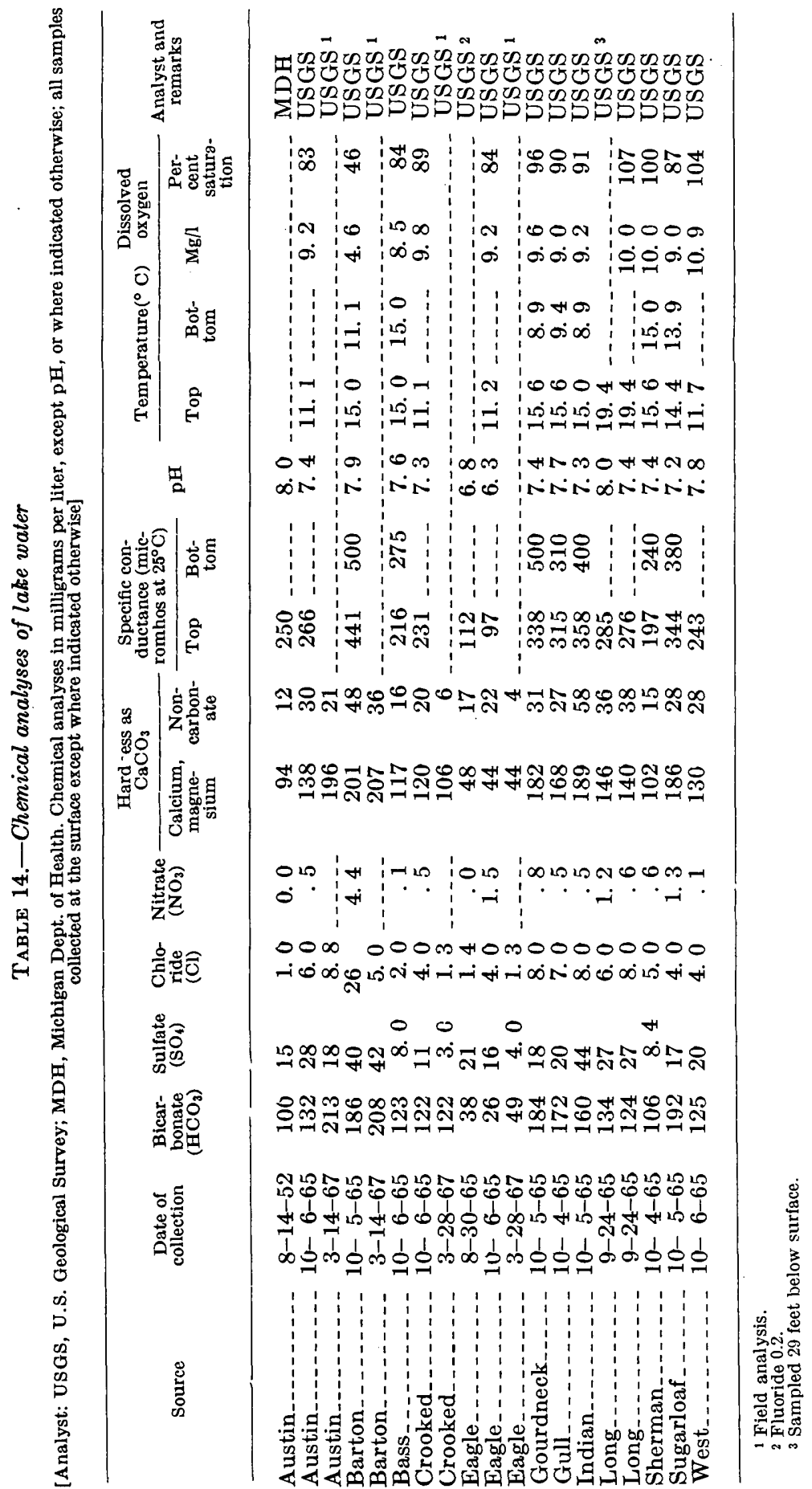


EAGLE AND CROOKED LAKES

Eagle and Crooked Lakes, located in the headwaters of West Fork Portage Creek basin, are unique in that their levels are maintained at high levels through the addition of water pumped from wells. This management practice allows lake associations to control, within certain limits, the level of the lakes. These lakes normally act as areas of ground-water recharge (indicated by their very low concentration of dissolved solids, table 14) and do not receive much inflow from ground water.

The addition of water from wells into Crooked and Eagle Lakes creates a higher head, forcing additional water to seep through the lake bottom into the upper aquifer. It also has two effects upon quality of the lake water. First, it has the direct effect of introducing water with a higher dissolved-solid content into the lake. This mineralization does not appear to reduce recreational values, but the lake water would continue to increase in mineral content. The second and more beneficial quality aspect is that the higher head retards the inflow of nutrient-rich effluent from surrounding septic tanks. This reduces aquatic growth which is detrimental to recreation and esthetic values.

\section{GULL LAKE}

Gull Lake is the longest and deepest (110 feet) in the county. Its level is controlled by a dam at the south end of the lake. Raising the natural level of the lake has altered the ground-water gradient in areas adjacent to the lake and has caused some water to move out of the south end of the lake by ground-water underflow. Subsurface outflow prevents septic-tank effluent from entering the lake at the south end. Storage of water in Gull Lake has also affected the chemical quality of water in Gull Creek (pl.6). The dissolved solids and hardness are lower than in the adjacent geologically similar Augusta Creek basin. The concentrations of these materials in Gull Creek increase as water flows downstream, until at the mouth the chemical quality is nearly the same as in Augusta Creek.

The maximum observed annual range of stage for Gull Lake was 1.14 feet. Because the lake level is relatively stable, the continued recreational value of Gull Lake is dependent almost entirely upon the quality of its water and related growth of vegetation in the lake. This in turn primarily depends upon the amount of nutrients contributed to the lake. The relatively small agricultural use of land in the basin, coupled with the small ratio of land area to water volume, results in very little nutrient-rich effluent. The major source 
of effluent is from domestic wastes. Therefore, the recreational future of Gull Lake is dependent largely upon the control of domestic wastes.

\section{SHERMAN LAKE}

Sherman Lake, immediately south of Gull Lake, has no inlet or outlet. As a consequence, its level is a direct function of the level of the water table, which in turn is dependent upon the rate of precipitation and evaporation. On the basis of the results of a pumping test and the sandy nature of the surrounding material, the hydrologic connection between the lake and the ground-water reservoir apparently is good. The low amount of dissolved solids in the water (table 14) indicates that the lake probably serves as a local source of recharge to the ground-water reservoir. Because this lake has a relatively small surface-drainage area, the amount of surface-water runoff containing agricultural fertilizer entering the lake is small. Because it is a ground-water recharge area, nutrient contribution from septic tank effluent to the lake probably is small also. Thus, the lake should retain its present high quality of water under present use.

\section{BARTON LAKE}

Barton Lake is in a degraded condition. The chemical quality of water in this lake is controlled by the quality of water from Portage Creek above Barton Lake and from a small, but significant, unnamed tributary near Vicksburg herein called Howard Lake Inlet (0972.15). The quality of Portage Creek has been degraded by the discharge of industrial and municipal effluents. Analyses from station 0972.05, upstream from Vicksburg, and station 0972.10 downstream from the city, show a substantial increase in chloride content and specific conductance in this reach (table 11).

Howard Lake Inlet has a relatively small flow but contains large amounts of nitrate (table 11). The source of this constituent is believed to be decaying vegetation in the marshy areas of this basin.

The concentration of dissolved oxygen in Barton Lake on September 5,1965 , was less than one-half of the amount that the water was capable of holding (table 14). The $4.6 \mathrm{mg} / \mathrm{l}$ of dissolved oxygen measured is below the generally accepted value of $5.0 \mathrm{mg} / \mathrm{l}$ for intolerant warm-water fish species (Michigan Water Resources Commission, 1968). The low oxygen content indicates that oxygen is being consumed as the waste effluents in the water are being assimilated by the biota in the lake. 
INDIAN LAKE

The elevation of Indian Lake is maintained by inflow from Portage River and Dorrence Creek. Because of the variable flow from these two sources, the lake has a rather large annual range in stage. The largest range observed, 3.30 feet, occurred in 1947 (table 12). Water in the lake is of good chemical quality. It is low in nitrates and has a high dissolved oxygen content. Possible future changes in the quality of this water are of some concern. Both Portage River and Dorrence Creek have high nitrate concentrations (table 11). The amount of nitrate in these streams depends to a large extent on the amount of fertilizer applied, slope of the land, precipitation intensity, and soil type. The rolling land and heavy clayey soil of most of the watershed above Indian Lake contribute to the high nutrient runoff. Nitrates when associated with phosphorus can cause excessive plant growth. Unless agricultural practices change, the lake will probably exhibit an increase in plant growth in the future.

\section{GROUND-WATER RESERVOIRS}

Ground water in quantities adequate to supply domestic and other relatively small needs is available at almost any place in Kalamazoo County. Ground water is available for large development where thick deposits of permeable materials of large areal extent form ground-water reservoirs.

One of the principal objectives of this study was to determine the perennial yield of these reservoirs. This yield is supplied by (1) recharge from precipitation, (2) ground water that moves into the reservoir from surrounding areas, and (3) infiltration of water from streams, natural ponds, and artificial recharge basins in the reservoir area.

In analyzing the potential yield of ground-water reservoirs, it must be recognized that recharge from precipitation is not at a constant rate. The hydrologic budgets for the Kalamazoo and St. Joseph River basins (tables 2 and 3) show that recharge occurs during the winter and spring months; in some years there is little recharge during the 6-month period June through November. In analyzing the yield of a ground-water reservoir it is assumed that no recharge will occur during a certain period. In humid areas, such as Michigan, periods of no recharge for 90 to 365 days have been assumed in various studies. A period of 180 days is probably most realistic, and such a period is used in the following analysis 
In estimating recharge from precipitation on the reservoir catchment areas in Kalamazoo County, water-table contours (pl. 4) are used to define the areas. It is assumed that all reservoirs are fully developed so that potential recharge is restricted to the area of diversion for each reservoir.

Movement of water into the ground-water reservoirs from surrounding areas is continuous in time but not in rate. The rate depends upon the hydraulic gradient, which is generally greatest in the spring when the water table or piezometric surface is highest. Because some of the reservoirs in Kalamazoo County are deeply buried, not all the potential recharge can be diverted into the area of influence caused by pumping; some of the recharge will move laterally in the shallow aquifer to areas of discharge without reaching the lower aquifer. Also, much of the recharge will move from the part of the aquifer upgradient from the area of pumping.

Infiltration of water to the ground-water reservoirs from streams, ponds, and artificial recharge basins occurs when the deposits between the surface source and the reservoir are permeable and there is a hydraulic gradient between the surface source and the reservoir. Generally, much of the infiltration occurs in the spring when streams and lakes are at high stage and there is a better connection with the ground-water reservoir. However, in artificial recharge basins, the time and amount of recharge can be regulated by man.

The relationship of ground-water reservoirs to pumping centers, well fields, and the average rate of pumpage at the pumping centers in 1966, as well as the chemical quality of water, is shown on plate 8 . In the illustration, well fields within a small area were grouped together to form pumping centers. Most of them are located in the Kalamazoo River and the Kalamazoo-Portage ground-water reservoirs. The data used in preparing plate 8 were collected as part of an inventory of water use in 1966. The reliability of pumpage data varies greatly. Generally, municipalities meter their water and maintain systematic pumpage records; only a small part of the industrial supply is metered. Where not metered, industrial pumpage data are estimates based on the number and capacity of pumps and the number of hours per day each pump operates. Therefore, these data range in accuracy from excellent to highly questionable. Because the records of wells and well fields have been totaled by pumping centers, they are probably fairly representative.

It is apparent from the preceding discussion that the yield of ground-water reservoirs involves several factors, all of which vary 
with time. Estimates of the yield can be made by several different methods, including analog and mathematical models of varying complexity. The selection of a particular method is dependent upon the nature of the available data, the time and funds available, and the degree of accuracy required. Careful consideration of the geology and hydrology in Kalamazoo County indicates that estimates of yield, sufficiently accurate for most purposes, can be obtained with the help of a relatively simple mathematical model.

The principal features of the mathematical model are an array of pumping wells, a distance-drawdown graph, and in the Kalamazoo River ground-water reservoir, a cross section. A hypothetical array of pumping wells is assumed with yields and drawdowns that will not dewater screens. Discharging image wells are introduced to compensate for the effects of aquifer boundaries (Ferris and others, 1962, p. 146151). It is assumed also that image wells are pumped at the same time and the same rate as the pumping wells. The semilogarithmic distancedrawdown graph is constructed with the hydraulic properties of the aquifer using the Theis nonequilibrium equation (Ferris and others, 1962 , p. 92-98). The curve from the graph can be used to determine the computed drawdown at the pumping wells and along lines connecting them. The computed drawdown consists of the sum of the drawdowns of the pumping wells and the image wells within an effective radius of the pumping wells. In upper aquifer reservoirs, the computed drawdown is adjusted for dewatering, using a dewatering, equation derived by Jacob (1944):

$$
s=s^{\prime}-\left({s^{\prime}}^{2} / 2 m\right)
$$

where $s=$ drawdown that would occur in an equivalent nonleaky artesian aquifer, in feet,

$s^{\prime}=$ observed drawdown under water-table conditions, in feet, and

$m=$ initial saturated thickness of aquifer, in feet.

The above analysis assumes that the only factors affecting drawdown are related to the aquifer. However, two other factors related to well design, partial penetration and well loss, can result in increased drawdowns unless wells are properly constructed and developed.

Several differences obviously had to be considered in creating the mathematical models for the Kalamazoo ground-water reservoirs. A. comparison is made of how model conditions differ from field conditions in order that the reader is better able to appraise the reliability 
of the final yield estimates. The most significant differences are compared below.

\section{Model conditions}

Reservoir boundaries are regular and impervious.

Reservoirs are not hydraulically connected.

Hydraulic properties are uniform

Water table is flat; no discharge to stream; no drawdowns from previous pumping.

No stream infiltration

Pumpage rates are fixed

No recharge from precipitation for 180 d.ays.

\section{Field conditions}

Keservoir boundaries are irregular and not impervious.

Some of the reservoirs may be hydraulically connected.

Hydraulic properties are highly variable near boundaries and locally, but are fairly uniform on an overall basis.

Water table slopes; discharge is to streams : drawdowns may be recovering from previous pumping.

Stream infiltration may reduce drawdowns in nearby wells.

Pumpage rates may vary with water needs.

Periods of no recharge from precipitation vary in length and in time of occurrence.

Although model conditions are at variance with actual field conditions, models still are applicable in estimating the amount of ground water potentially available. Allowances have been made in discussions of the individual ground-water reservoirs to account for differences such as recharge from precipitation, stream infiltration, and reservoir boundaries. Therefore, use of the mathematical model will not introduce serious errors in providing approximate answers that may be applied to the practical problems of reservoir development in Kalamazoo County.

\section{KALAMAZOO RIVER GROUND-WATER RESERVOIR}

Thick saturated deposits of coarse sand and gravel in the Kalamazoo River valley form an upper aquifer. The aquifer is especially thick near the river in Kalamazoo Township. As indicated in section $\mathbf{A}-\mathbf{A}^{\prime}$ and shown by isopach lines on plate 1 , it has a thickness of more than 140 feet. Along the Kalamazoo River, from about the east boundary to the north boundary of Kalamazoo Township, thicknesses range from 40 to 140 feet, respectively. In most of this area, the coefficients of transmissibility range from about 20,000 to 120,000 gpd per foot (pl. 2). The part of the upper aquifer where the saturated thickness is 40 feet or greater and the transmissibility is 40,000 gpd per foot or more constitutes the Kalamazoo River ground-water reservoir. It is connected with the Cooper groundwater reservoir to the north. In order to simplify the reservoir for 
model analysis, it is assumed that the reservoir is a rectangular area bounded by straight, parallel boundaries; consequently, it may include minor areas of saturated thickness and transmissibility less than that described above. These boundaries form the rectangular reservoir shown on plate 2 .

\section{IDEALIZED RESERVOIR}

The model aquifer selected for the Kalamazoo River ground-water reservoir is a rectilinear strip of coarse sand and gravel, 16,000 feet wide, 27,000 feet long, and 60 feet thick, bounded on the sides and bottom by impermeable material. The model assumes no stream infiltration. The weighted average coefficients of transmissibility and storage are 60,000 gpd per foot and 0.20 , respectively. Pumping tests (table 1) and geologic data were used in selecting an average storage coefficient, which was considered to be representative of the aquifer for long periods of continuous withdrawal.

Figure 26 shows the idealized Kalamazoo River ground-water reservoir with 36 hypothetical pumping wells, each pumping at a rate of $750 \mathrm{gpm}$ or a total of $39 \mathrm{mgd}$ for 180 days without recharge. $W(u)$ is the well function of $u$, a constant discharge situation (figs. 26, 28, and 29). Computations made with the distancedrawdown curve indicate maximum drawdowns would be about 50 feet at the pumping wells. Drawdowns caused by a single pumping well would be negligible beyond 6,000 feet.

WATER POTENTIALLY AVAILABLE TO THE RESERVOIR

Precipitation percolating to the water table recharges the Kalamazoo River ground-water reservoir. Pumping of wells near the Kalamazoo River and several small tributary streams lowers water levels so that appreciable quantities of water move from the river to the reservoir by the process of induced infiltration. Even if water levels are not lowered below river level, the pumping reduces the natural discharge to the river. In either case the net result is reduction of streamflow. The average annual rate of ground-water recharge directly to the reservoir from precipitation was estimated to be 9 inches (Kalamazoo River basin, p. 17). The area that supplies recharge to the reservoir is estimated to be $40 \mathrm{sq} \mathrm{mi}$, including an area of $25 \mathrm{sq} \mathrm{mi}$ outside the reservoir. Therefore, the average rate of recharge from precipitation is about $17 \mathrm{mgd}$. Thus, to maintain a hypothetical draft rate of $39 \mathrm{mgd}$, 'utilizing all the recharge from precipitation, it would be necessary to induce recharge from the Kalamazoo River and other tributary streams at a rate of 22 mgd. Rates at which the Kalamazoo River and other streams would 


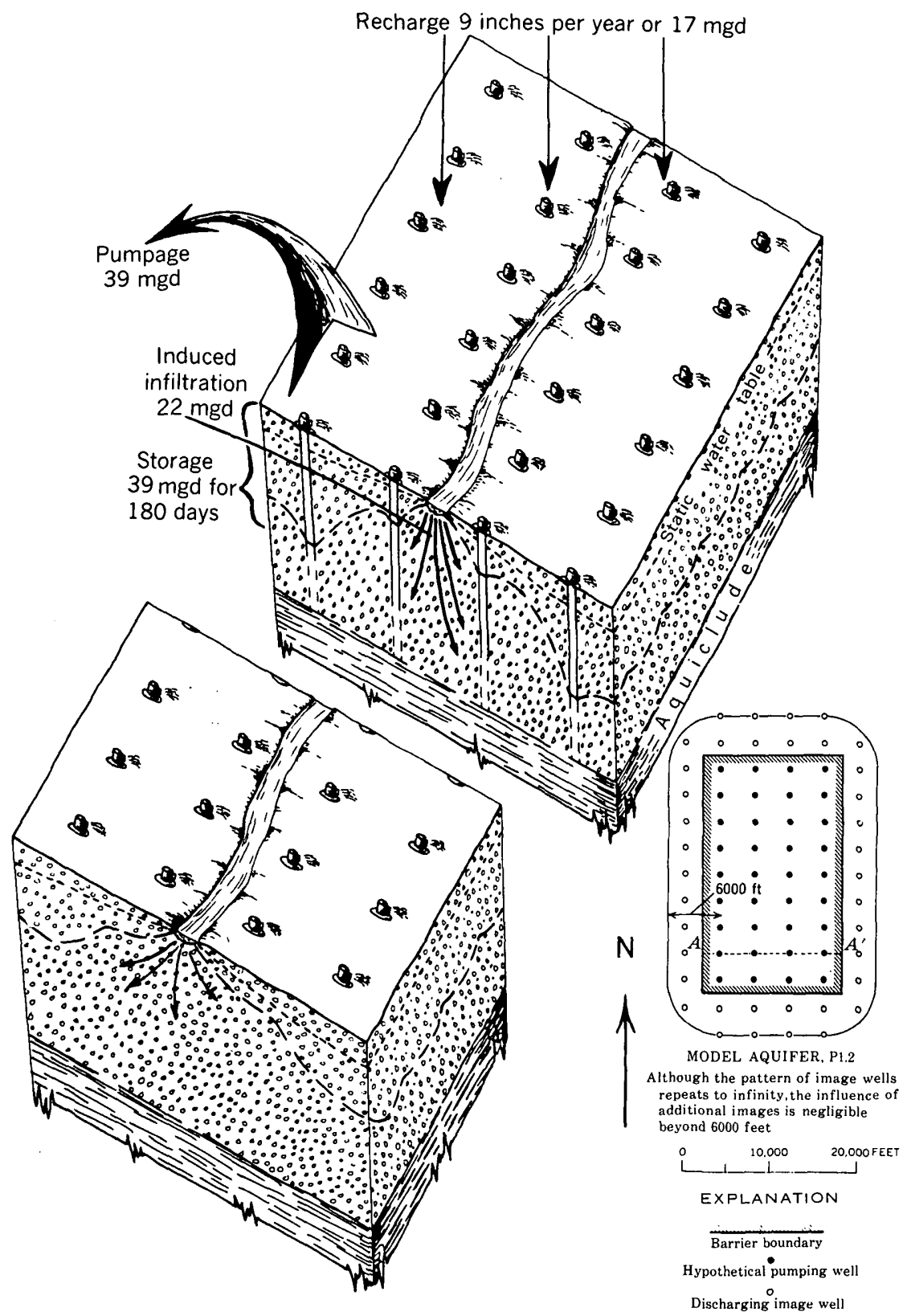

Fiaure 26.-Mathematical model of Kalamazoo River ground-water reservoir County, south- 
recharge the ground-water reservoir by induced infiltration could not be determined because the extent to which river infiltration is impeded by natural and artificial sealing of the river sides and bottom is not known. Also, scraping and dredging-methods of effectively maintaining hydraulic connection between the river and the reservoir-are now common practices, and more such practices are contemplated for the future.

In order to estimate the minimum amount of water that could be infiltrated from the stream to the reservoir, discharge measurements made on the Kalamazoo River and its tributaries during the seepage run of July 27 and 28,1964 - a time when the streamflow was very low-were examined. Discharges in streams overlying the reservoir were estimated and tabulated in a surface-water budget. It was assumed that the flow estimated for Portage and Allen Creeks does not include water now (1966) withdrawn or water potentially to be withdrawn as a part of the development of the Kalamazoo-Portage reservoir. Flows of other tributaries were assumed to be a part of
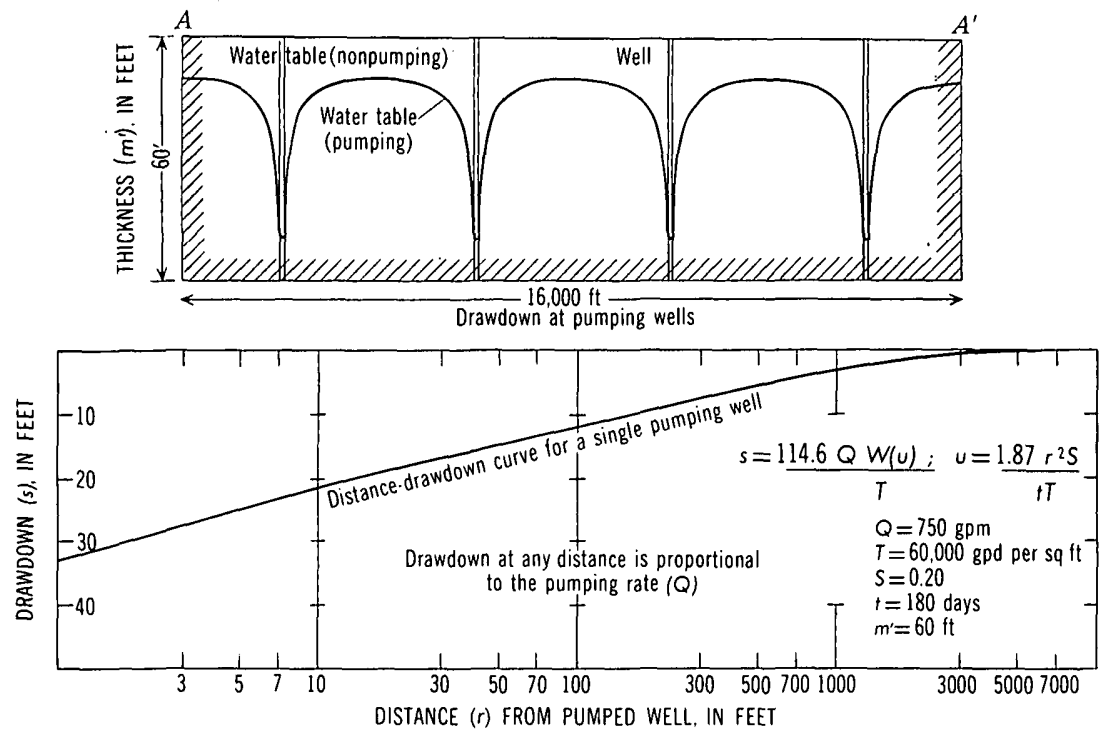

and idealized diagram showing practical scheme of development, Kalamazoo western Michigan. 
recharge from precipitation and were utilized in reservoir development. The sites of the discharge measurements are shown on plate 5 .

Summary of surface-water budget of Kalamazoo River ground-water reservoir to show stream infiltration

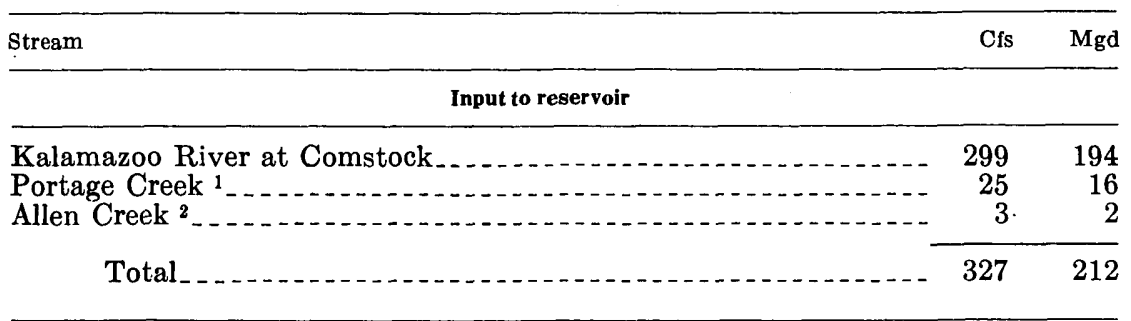

Output from reservoir

Kalamazoo River ${ }^{8} \ldots \ldots \ldots 10$

Stream infiltration needed to balance budget.

Total . . . . .

1 Estimated from Portage Creek near Kalamazoo.

Estimated from Allen Creek at Kalamazoo.

3 Estimated from Kalamazoo River at D Avenue.

In 1964, the estimated average rate of pumping in the Kalamazoo River ground-water reservoir was $27 \mathrm{mgd}$ (table 15). Stream infiltration, as shown above, could furnish at least $11 \mathrm{mgd}$ and groundwater storage probably furnished the remaining $16 \mathrm{mgd}$ (recharge from precipitation was negligible during most of 1964). Thus, it may be assumed that about 40 percent of the water pumped was from stream infiltration and that the remaining 60 percent was from ground-water storage that was replaced by recharge from precipitation over several years.

In order to estimate the maximum amount of water that could be infiltrated from unsilted streams to the Kalamazoo River groundwater reservoir, it was assumed that there was an average streambed width of 25 feet, a length of 1,000 feet of stream, and a difference in vertical head of 1 foot. Estimating the vertical permeability to be $16 \mathrm{gpd}$ per sq $\mathrm{ft}$, the infiltration capacity would be about $400,000 \mathrm{gpd}$. Thus, with a 60,000 foot reach of the Kalamazoo River and other tributary streams overlying the reservoir, the infiltration capacity would be $24 \mathrm{mgd}$ or at about 11 percent of the low flow. To withdraw a maximum of $24 \mathrm{mgd}$ from stream infiltration, it would be necessary to place closely spaced wells along the entire length of all the streams.

Ground-water storage in the Kalamazoo River ground-water reservoir permits pumping at rates greater than recharge from precipitation and stream infiltration for limited periods. However, the prac- 
tical rate of withdrawal from storage considers the amount of drawdown that allows sufficient water left in storage to provide head to move water toward the wells. In the mathematical model, the practical rate of withdrawal from storage would be limited to $39 \mathrm{mgd}$ for 180 days without recharge.

\section{PRACTICAL DEVELOPMENT OF EXISTING PUMPING CENTERS}

In 1966, water was being withdrawn from well fields in pumping centers A, B, and C of the Kalamazoo River ground-water reservoir at a rate of about $28 \mathrm{mgd}$. Water levels in wells within and outside the cones of depression formed by the pumping centers have been measured intermittently since 1946. Hydrographs of water-level fluctuations, pumpage, and precipitation for the period 1946-66 were examined in order to determine a practical limit of development for the pumping centers (fig. 27). The hydrographs showed that there were periods when withdrawals exceeded recharge and ground-water storage was depleted, periods when recharge exceeded withdrawals and ground-water storage was replenished, and periods when recharge balanced withdrawals and ground-water storage remained unchanged. By comparison of these periods, the following potential increase in rate of pumpage, in million gallons per day, was determined.

\begin{tabular}{|c|c|c|c|}
\hline Pumping center & $\begin{array}{l}\text { Average } \\
\text { daily plumpage } \\
\text { in } 1966\end{array}$ & $\begin{array}{l}\text { Potential } \\
\text { increase in } \\
\text { pumpage }\end{array}$ & $\begin{array}{c}\text { Practical } \\
\text { limit of } \\
\text { development }\end{array}$ \\
\hline 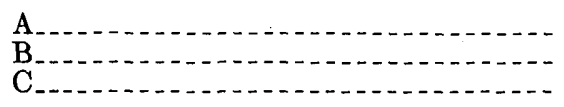 & $\begin{array}{r}\text { 10. } 85 \\
7.12 \\
9.78\end{array}$ & $\begin{aligned} * 4.15 \\
2.88 \\
* 4.22\end{aligned}$ & $\begin{array}{l}15 \\
10 \\
14\end{array}$ \\
\hline Total & 27.75 & 11. 25 & 39 \\
\hline
\end{tabular}

*Assuming a good connection with the Kalamazioo River.

\section{DEVELOPMENT OF THE RESERVOIR}

Figure 26 is an idealized diagram showing the potential yield of the Kalamazoo River ground-water reservoir with a selected scheme of development utilizing stream infiltration. The potential yield of the reservoir is defined as the maximum amount of water that can be continuously withdrawn with a selected system of wells without creating critical drawdowns or exceeding recharge. With the pumping wells spaced in the general pattern shown, withdrawal of $39 \mathrm{mgd}$ would have drawdowns no greater than 50 feet and recharge of 17 mgd from precipitation and $22 \mathrm{mgd}$ from stream infiltration. Recharge from precipitation on the reservoir catchment area could be captured by the outer row of wells; that from stream infiltration by 


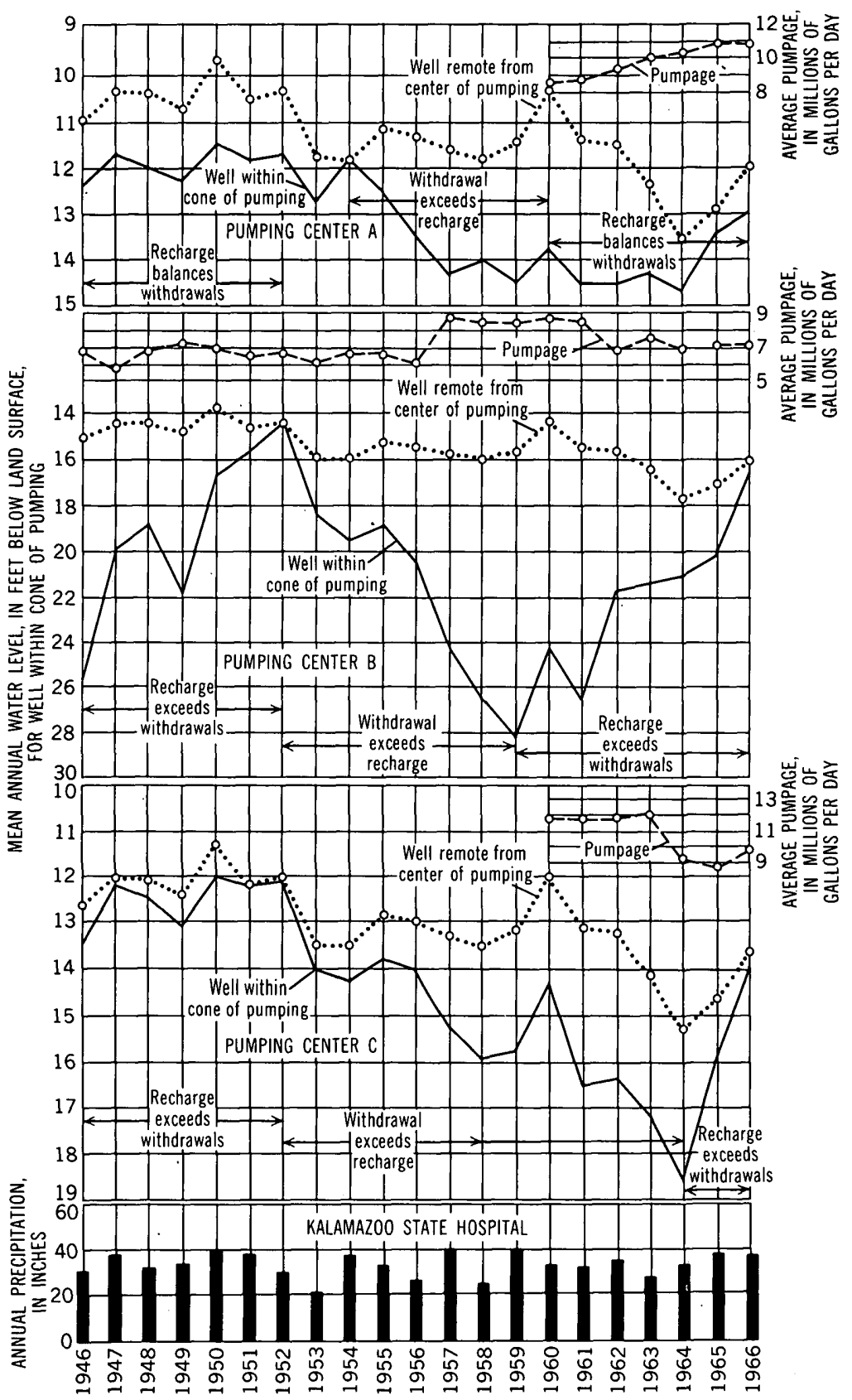

FIgure 27.--Relationship between water-level fluctuations, pumpage, and precipitation at observation wells from the Kalamazoo River groundwater reservoir, 1946-66. 
the inner row of wells. Deficiencies in recharge during periods of drought could be supplemented by ground-water storage.

Although many schemes could be selected for development of the Kalamazoo River ground-water reservoir, the one chosen is believed to be a practical scheme of development. Greater utilization of stream infiltration under present (1968) conditions appears undesirable. Water in the Kalamazoo River and its tributaries is of relatively high dissolved-solids content and potential infiltration rates are unknown. Withdrawal from storage could be as much as $39 \mathrm{mgd}$ for 180 days. The $22 \mathrm{mgd}$ assumed replenished by stream infiltration is only about 10 percent of the 7-day low flow of $213 \mathrm{mgd}$ or the dependable supply in the Kalamazoo River and would have little effect on higher flows. By placing more wells close to the Kalamazoo River and tributary streams or by pumping with higher yields than in the model, it would be possible to induce more water from the streams. This was not done in the idealized scheme of development because, as mentioned above, water in the Kalamazoo River and other streams is of questionable quality, and potential infiltration rates are unknown.

\section{CHEMICAL QUALITY}

Representative chemical constituents from specific wells, well fields, and pumping centers are shown graphically, on plate 8 . Some of the graphs represent individual analyses, and others represent averages of several analyses. At some of the well fields in pumping centers $\mathbf{A}$ and $\mathrm{C}$ in the Kalamazoo River ground-water reservoir, the chemical constituents are influenced by infiltration of water of relatively high dissolved-solids content from the Kalamazoo River, its tributaries, and seepage from stockpiles of industrial minerals. At the city of Kalamazoo, Station 5 well field, in pumping center C, the relatively high dissolved-solids content shown on the graph is probably caused by infiltration of water having a high dissolved-solids content from a small unnamed tributary of the Kalamazoo River. At well fields in pumping center $B$ in the Kalamazoo River ground-water reservoir, the chemical constituents are probably influenced by infiltration of water from Axtell and Portage Creeks. Both creeks are reported to have a relatively high concentration of dissolved solids. Thus, the chemical quality of water will affect the usefulness and the quantities of water available for development in the Kalamazoo River groundwater reservoir.

\section{SCHOOLCRAFT GROUND-WATER RESERVOIR}

A large ground-water reservoir underlies most of Schoolcraft Township. Coarse sand and gravel beds, separated by beds of very fine 
sand, silt, clay, and till deposits, make up an upper and a lower aquifer. The middle unit functions as a leaky aquiclude or semiconfining layer which impedes the vertical movement of water from the upper aquifer to the lower. The units are shown along the line of section $C C^{\prime}$ on plate 1 . To the west and south, the lower aquifer grades into very fine sand, silt, and clay; to the east, it thins and grades into the lower aquifer of the Vicksburg ground-water reservoir; and to the north, it thins and grades into the lower aquifer of the Kalamazoo-Portage ground-water reservoir. The upper aquifer is about 80 feet thick on the west and thins to the east to about 20 feet. For most of the reservoir, the coefficient of transmissibility of the upper aquifer ranges from about 40,000 to $80,000 \mathrm{gpd}$ per foot; that of the lower aquifer ranges from about 10,000 to 100,000 gpd per foot. (See pl. 2.)

\section{IDEALIZED RESERVOIR}

The Schoolcraft ground-water reservoir was considered as an idealized model using criteria similar to those of the Kalamazoo River ground-water reservoir. The model is a rectangular area 20,000 feet wide by 40,000 feet long with straight, parallel impermeable boundaries. The upper aquifer has an average thickness of 40 feet; the lower aquifer, 80 feet; and the aquiclude, 30 feet. Coefficients of transmissibility of 40,000 and 80,000 gpd per foot and storage coefficients of 0.20 and 0.005 were used as weighted averages for the upper and lower aquifers, respectively. The vertical permeability of the aquiclude was estimated to be 0.05 gpd per square foot. No stream infiltration was considered. An assumed configuration of 24 wells pumping from the lower aquifer at a rate of $500 \mathrm{gpm}$ each or $17 \mathrm{mgd}$ is used for model analysis (fig. 28). Maximum available drawdowns at the pumping wells were assumed to be about 70 feet (to the top of lower aquifer). Computations, using the distance-drawdown curve, indicate that $17 \mathrm{mgd}$ can be withdrawn for 180 days with maximum drawdowns of only 27 feet. Drawdown caused by a single pumping well is negligible beyond 30,000 feet.

\section{WATER POTENTIALLY AVAILABLE TO THE RESERVOIR}

The hydrologic features that affect the water potentially available to the Schoolcraft ground-water reservoir are recharge from precipitation, storage, and vertical leakage from upper to lower aquifers. No stream infiltration is considered. Recharge, derived chiefly from precipitation by the vertical leakage of water through the confining bed, must be available to replenish the assumed rate of withdrawal of 17 mgd from the model. Assuming an average recharge rate of 9 inches per year (St. Joseph River basin, p. 17) on an area of about $43 \mathrm{sq} \mathrm{mi}$, 
including an area of $11 \mathrm{sq}$ mi outside the reservoir, the average recharge would be approximately $18 \mathrm{mgd}$ or more than enough to support the yield of $17 \mathrm{mgd}$. Reservoir storage of the upper aquifer could provide an estimated withdrawal rate of $17 \mathrm{mgd}$ for a' 180 day period without recharge. Leakage occurs through the aquiclude because of the difference in head between the water table in the upper aquifer and the piezometric surface of the lower aquifer. Leakage must be adequate to replace water withdrawn from the lower aquifer in the idealized reservoir. Under the assumed model conditions, the potential rate of leakage through the aquiclude was $18 \mathrm{mgd}$ using an estimated average head difference of 12 feet in the modified form of the Darcy equation,

$$
Q=\left(P^{\prime} / m^{\prime}\right) \Delta h A,
$$

where $Q=$ rate of leakage in million gallons per day,

$P^{\prime}=$ vertical permeability,

$m^{\prime}=$ thickness of confining bed in feet,

$\Delta h=$ head difference in feet, and

$A=$ area in square feet.

The leakage rate of $18 \mathrm{mgd}$ is more than adequate to offset the yield of $17 \mathrm{mgd}$. Long-term yield is a function of leakage only if maximum leakage rate is less than the recharge. Thus, there is water potentially available to the model reservoir to meet the assumed rate of withdrawal and drawdown.

\section{FUTURE DEVELOPMENT OF THE RESERVOIR}

At present (1968), the Schoolcraft ground-water reservoir is relatively undeveloped. The only withdrawals are from well fields of the cities of Portage and Schoolcraft, which pumped together an average of $0.19 \mathrm{mgd}$ in 1966. Average pumpage from the Schoolcraft reservoir for 1960 through 1966 is tabulated in table 15 and showis only a slight increase. As indicated above, the potential development of the reservoir is estimated to be about $17 \mathrm{mgd}$. This yield is about 90 times the actual rate of withdrawal in 1966. Thus, there are large untapped supplies of ground water available for future development in the Schoolcraft ground-water reservoir. Observation wells should be established to show future trends in withdrawals.

The idealized arrangement of wells used for the model aquifer of the Schoolcraft ground-water reservoir is not that suggested for practical development of the reservoir. Any development of wells or well fields should consider the competing use of surface water in this area. If $17 \mathrm{mgd}$ were withdrawn by wells, almost all the groundwater recharge would be intercepted; and the principal stream, Gourdneck Creek, which receives part of its flow from ground-water 
runoff, would be dry part of the year. The levels of lakes in the Austin Lake complex would also be seriously affected and water would not be available to the proposed Portage-Gourdneck Creeks

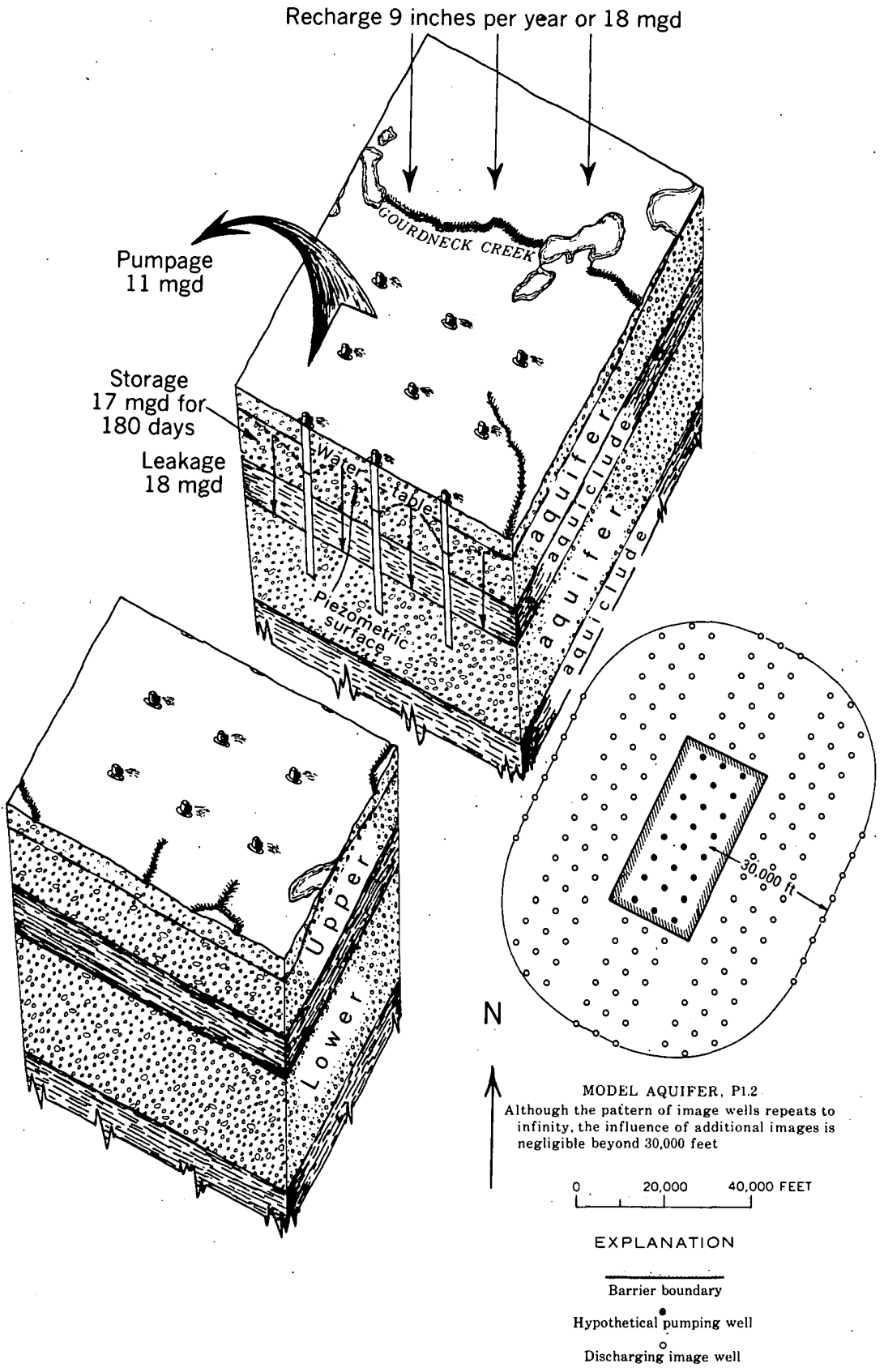

Figure 28.-Mathematical model of Schoolcraft ground-water 
surface-water reservoir. Thus, the competing use of surface water precludes the development of the entire recharge. from precipitation.

Figure 28 is an idealized diagram to show the potential yield of the Schoolcraft ground-water reservoir with a modified (practical) scheme of development. To avoid conflict of use with presently developed surface-water resources within the county, only the southern twothirds of the reservior would be developed. With 15 wells spaced and located as shown and pumping at $500 \mathrm{gpm}$ each, a withdrawal of about $11 \mathrm{mgd}$ would cause maximum drawdowns of only 27 feet at pumping wells. Rates of withdrawal greater than $11 \mathrm{mgd}$ would exceed the long-term rate of recharge from the reduced area. Taking into consideration the competitive use of surface water in the area, the practical scheme of development is considered to be a conservative approach to a potential yield of the ground-water reservoir.

The Schoolcraft ground-water reservior contains water that is suitable for most uses; however, it is very hard and locally contains objectionable quantities of iron. A comparison of the three graphs on plate 8 shows the water to have a fairly uniform chemical composition.

\section{KALAMAZOO-PORTAGE. GROUND-WATER RESERVOIR}

Part of the cities of Kalamazoo and Portage are underlain by a large ground-water reservoir called the Kalamazoo-Portage groundwater reservoir. Coarse sand and gravel beds form an upper aquifer and two lower aquifers, which are separated by beds of very fine sand, silt, clay, and till of low permeability. The separating beds form leaky aquicludes or semiconfining layers that impede the vertical movement of water from the upper to the lower aquifer and also between the two lower aquifers. The positions of the aquifers and aquicludes of the

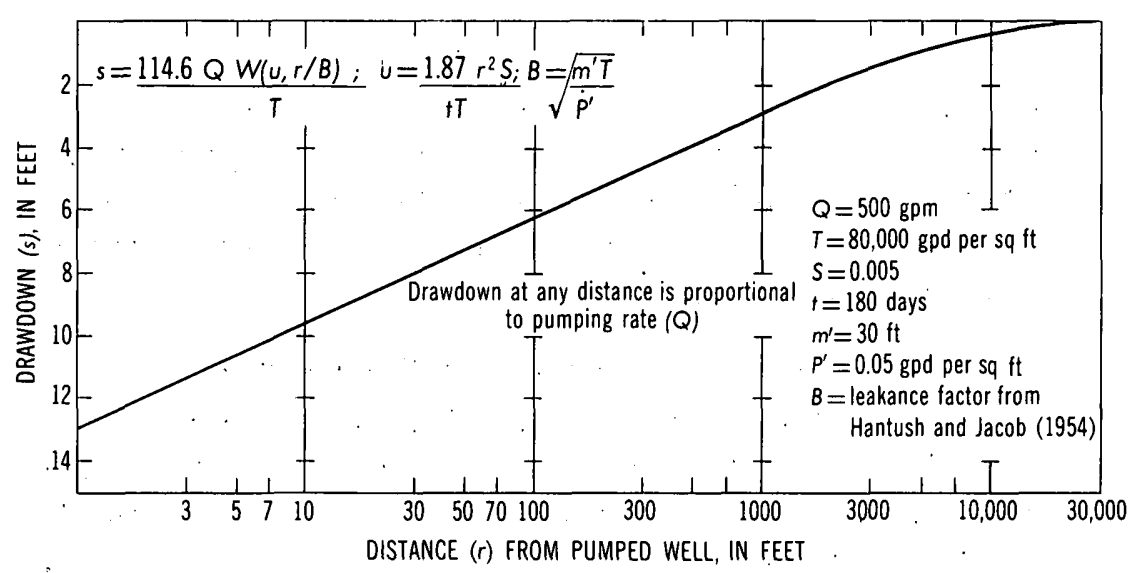

reservoir and idealized diagram showing practical scheme of development. 
Kalamazoo-Portage ground-water reservoir are shown on sections $A-A^{\prime}$ and $B-B^{\prime}$ (pl. 1). From southwest to northeast, the upper aquifer decreases in thickness from about 60 to zero feet. The lower aquifers have a combined saturated thickness that averages about 70 feet. They are connected to the southwest with the Schoolcraft ground-water reservoir, to the west with the Texas ground-water reservoir, and to the north with the upper aquifer of the Kalamazoo River ground-water reservoir. In other directions, the lower aquifers grade entirely into aquicludes. For most of the reservoir, the transmissibility of the upper aquifer ranges from about 10,000 to 100,000 gpd per foot; those of the lower aquifers range from about 10,000 to 160,000 gpd per foot (pl. 2).

\section{IDEALIZED RESERVOIR}

The model of the Kalamazoo-Portage ground-water reservoir was idealized into a rectangular area 25,000 feet wide by 40,000 feet long. The upper aquifer in this reservoir has an average saturated thickness of 30 feet, the lower aquifer, 70 feet, and the upper aquiclude, 70 feet. Although an aquiclude as much as 100 feet thick separates the two lower aquifers in places, they are considered here as one lower aquifer 70 feet thick. Average coefficients of transmissibility of 60,000 and $80,000 \mathrm{gpd}$ per foot and average storage coefficients of 0.20 and 0.005 were estimated for the upper and lower aquifers, respectively. The vertical permeability of the aquiclude was estimated to be $0.05 \mathrm{gpd}$ per square foot. Twenty-eight assumed supply wells, pumping at a rate of $600 \mathrm{gpm}$ each, or a total of $24 \mathrm{mgd}$, were selected for the mathematical analysis of the model reservoir (fig. 29). Maximum drawdowns from the pumping wells should not be greater than 100 feet (the depth to the top of lower aquifer). Computations using the distance-drawdown curve indicate that withdrawals of $24 \mathrm{mgd}$ for 180 days without recharge can be obtained with drawdowns of 45 feet at the pumping wells. Drawdown caused by a single pumping well is negligible beyond 30,000 feet. Since the maximum assumed drawdown is 100 feet, the computed drawdowns of 45 feet are very conservative and the model reservoir can easily support the average withdrawal rate of $24 \mathrm{mgd}$ for 180 days without recharge.

\section{WATER POTENTIALLY AVAILABLE TO THE RESERVOIR}

Recharge to the lower aquifer is derived chiefly from precipitation, storage, and vertical leakage through the aquiclude (fig. 29). Assuming an average recharge rate of 9 inches per year on an estimated area of $63 \mathrm{sq} \mathrm{mi}$, including an area of $23 \mathrm{sq}$ mi outside the reservoir, the average annual recharge would be about $27 \mathrm{mgd}-$ more than 
enough to replenish the assumed withdrawal rate of $24 \mathrm{mgd}$ from the model. Storage in the upper aquifer is estimated to be adequate to support a withdrawal rate of about $24 \mathrm{mgd}$ for a 180-day period without recharge. Potential leakage through the upper aquiclude was estimated to be at a rate of about $27 \mathrm{mgd}$. Thus, the water potentially available to the lower aquifer could more than support a rate of withdrawal of $24 \mathrm{mgd}$. Stream infiltration could be used to recharge starage and reduce the rate and amount of drawdown.

PRACTICAL DEVELOPMENT OF EXISTING PUMPING GENTERS

In the Kalamazoo-Portage ground-water reservoir most of the water pumped is from the lower aquifer. There are six pumping centers that together withdrew an average of about $21 \mathrm{mgd}$ in 1966 . These pumping centers are fairly evenly distributed within the area overlying the ground-water reservoir (pl. 8). However, pumpage is not evenly distributed. For example, in 1966, pumping center F withdrew $12.77 \mathrm{mgd}$, or about 61 percent of the total pumpage, and pumping center $\mathrm{B}$ withdrew $0.77 \mathrm{mgd}$, or only about 4 percent of the pumpage. Most of the water withdrawn at pumping centers A through $\mathrm{E}$ is used for public supply, sewered, and removed from the ground-water reservoir area. From 60 to 80 percent of the water withdrawn at the Upjohn Co. in pumping center $F$ is diverted after use to artificial recharge basins, to Portage Creek, and since 1967, to Austin Lake. Therefore, this water is available to recharge the ground-water reservoir.

Observation wells are available within and outside the cones of depression at pumping centers $\mathrm{A}, \mathrm{E}$, and $\mathrm{F}$ to monitor the effects of pumping. Water-level records, including drawdown, are available from supply wells at pumping centers B, C, and D. They are evaluated to help estimate the practical limit of development for each of the six pumping centers.

At pumping center A, four well fields withdraw water from the ground-water reservoir. Monthly water-level fluctuations from wells within and outside the cone of depression formed by the pumping center, pumpage, and precipitation were graphed for the period 196366 (fig. 30). The relationship between water levels, pumpage, and precipitation shows that withdrawals of about $2.5 \mathrm{mgd}$ balance the recharge during the period 1963-66. It is estimated from the graphs that withdrawals probably can be at a rate of about $3 \mathrm{mgd}$ without seriously depleting storage and without adverse effects on other nearby pumping centers. Water levels and piezometric surfaces can be maintained at high stages by artificial recharge. Artificial storage ponds can store surface runoff and provide a more effective source of 
induced recharge than do existing lakes and ponds in the area because water levels in the artificial basins can be more easily controlled. Water-level measurements should be continued in the existing observation well in order to record pumping fluctuations and to monitor the effects of future withdrawals from the lower aquifer.

At pumping center $\mathrm{B}$, water-level records from observation wells were not available to determine long-term water-level trends. Two well fields withdraw water from the lower aquifer at this center. Before pumping started at the Station 4 well field of the city of Kalamazoo, wells were reported to flow and the piezometric surface was about 19 feet above land surface. About a mile away at the well field of Reliance Panelyte, Inc., water levels in wells not affected by pumping were reported to be about 7 feet below land surface. Water levels in pumping wells are reported to be 23 feet below land surface when the Station 4 wells are pumped at $600 \mathrm{gpm}$, and 84 feet below land surface, when the Panelyte wells are pumped at $920 \mathrm{gpm}$. Specific capacities of 14 and 12, respectively, computed for the two well fields imply that the drawdowns are probably affected by well loss. From 1960 through 1966 average pumpage for the center showed a slight increase (table 15). At the 1966 rate of pumping of $0.77 \mathrm{mgd}$, drawdowns, when corrected for well loss, would not appear to be excessive. However, the upper aquifer is missing and a large part of the water reaching the lower aquifer may be derived from natural recharge from adjacent areas. Much of the recharge is currently being utilized by nearby pumping centers. Based on the above facts, withdrawals probably should not be increased appreciably and the practical level

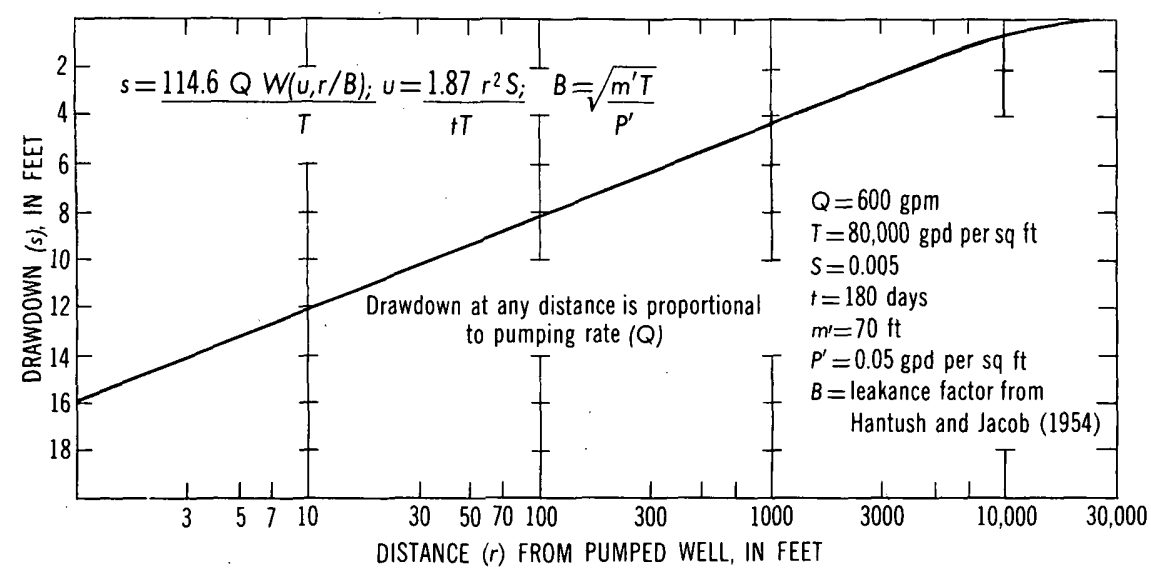

Figure 29.-Mathematical model of Kalamazoo-Portage ground-water 


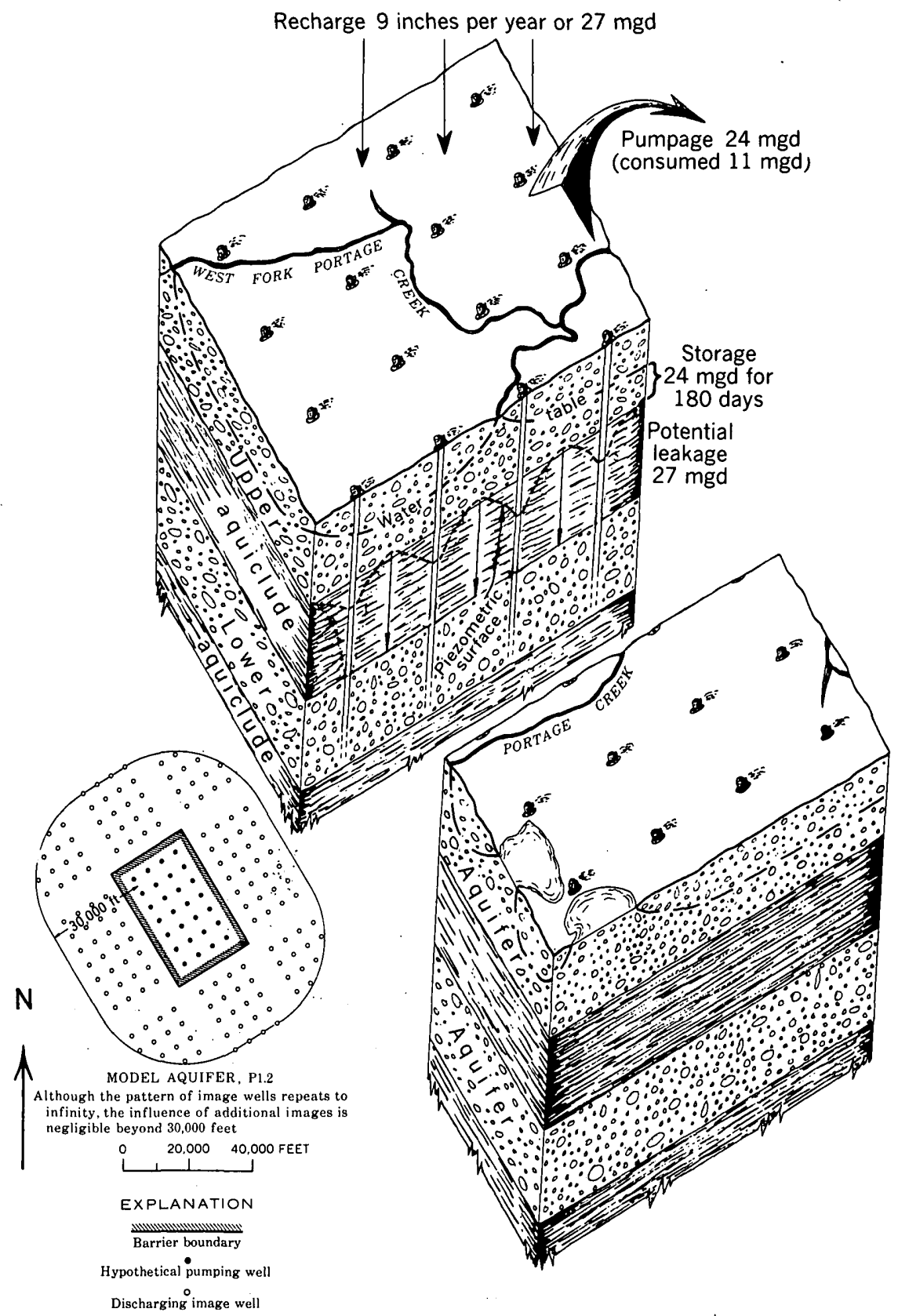

reservoir and idealized diagram showing practical scheme of development. 
WATER AVAILABILITY, KALAMAZOO COUNTY, MICHIGAN
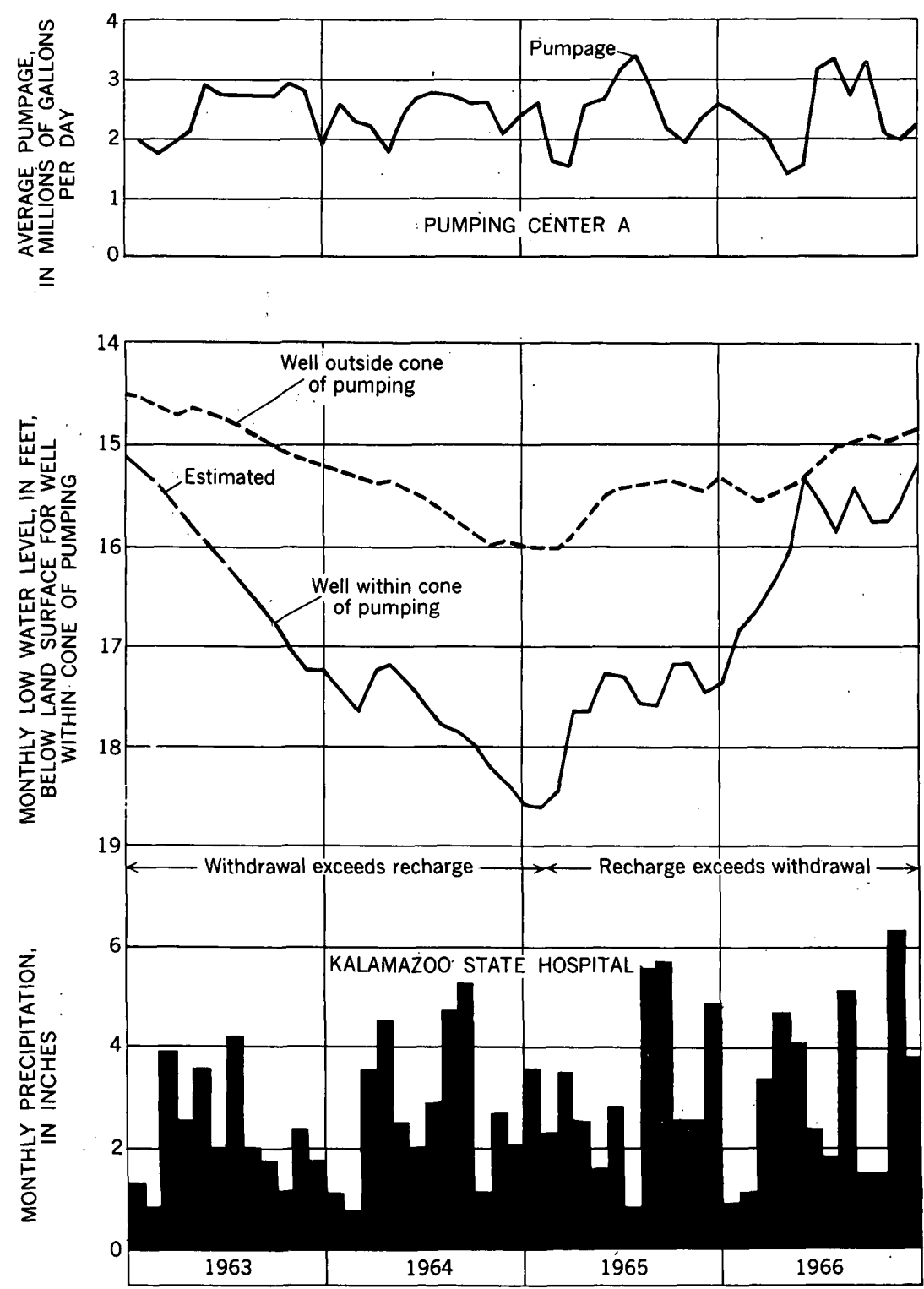

Figure 30.-Relationship between water-level fluctuations, pumpage, and precipitation at pumping center $A$ of the Kalamazoo-Portage groundwater reservoir, 1963-66.

of development should not be more than about $1 \mathrm{mgd}$. An observation well, deep enough to penetrate the lower aquifer, should be installed at the center to monitor future water-level fluctuations caused by pumping. 
The benefits of artificial recharge should be considered at pumping center B. Because the upper aquifer is missing, injection of water through the aquiclude by pits, shafts, or wells is a possibility. However, injection wells are costly and expensive to maintain and generally require the attention of highly trained specialists if they are to be operated successfully.

Pumping center $\mathrm{C}$ consists of six well fields with about $11 / 2$ miles between each well field. Only small amounts of water are withdrawn from each of the well fields. In wells not being pumped, static-water levels are reported to range from 2 to 47 feet below land surface. Drawdowns in pumping wells range from 9 feet at a pumping rate of $520 \mathrm{gpm}$ to 90 feet at $715 \mathrm{gpm}$. The average pumpage rate for the center has increased in the period 1960 through 1966 from 0.05 to $0.85 \mathrm{mgd}$ (table 15). Withdrawals from the lower aquifer are relatively small because there is no upper aquifer to store and transmit water to the lower aquifer. Withdrawals probably should be limited to an average rate of not more than $1 \mathrm{mgd}$ because of the low storage and transmittal potential. An observation well should be installed in the lower aquifer near the middle of the pumping center to monitor future water-level fluctuations caused by pumping.

Artificial recharge by means of injection pits, shafts, and wells could be practiced at pumping center $\mathrm{C}$ to increase the rate of transmittal of water to the lower aquifer.

Pumping center D consists of four well fields about 2 miles apart. The Station 22 well field of the city of Kalamazoo pumps most of the water withdrawn from the center. No observation wells have been used in the past to monitor long-term effects of changes in water levels due to withdrawals. In wells not being pumped, staticwater levels are reported to be from 38 to 64 feet below land surface. At a pumping rate of $1,000 \mathrm{gpm}$, drawdowns in pumping wells range from 10 to 50 feet but do not indicate depletion of supply. Pumpage at center D started in 1962 at an average rate of $0.03 \mathrm{mgd}$, increased by 1964 to $2.14 \mathrm{mgd}$, and then dropped off in 1965 and 1966 to 1.84 and $1.78 \mathrm{mgd}$, respectively (table 15). Withdrawals have induced water from West Fork Portage Creek to recharge the pumping center at estimated average rates of about $1 \mathrm{mgd}$. Because the upper and lower aquifers are thick and the coefficient of transmissibility is high (pls. 1,2), new well fields could be developed and withdrawals increased substantially. However, new well developments would reduce the movement of ground water to pumping center B. Thus, withdrawals probably should be at an average rate of not more than about $3 \mathrm{mgd}$. An observation well should be installed to penetrate the lower aquifer at the Station 22 
well field of the city of Kalamazoo to monitor the effects of future pumping.

Artificial recharge is possible by constructing storm-runoff detention basins at pumping center D. By deliberately drawing the water table down farther during periods of peak consumption, underground storage capacity can be created that will be filled subsequently by surplus storm runoff. The detention basin functions to collect and hold storm runoff that would otherwise run off unused.

There are four well fields at pumping center E. Annual waterlevel fluctuations from wells within and outside the cone of depression, pumpage, and precipitation are shown at the Station 9 well field of the city of Kalamazoo for the period 1960-66 (fig. 31). Wells located outside the cone of depression penetrate the upper aquifer; wells within the cone of depression penetrate the upper aquifer in the top hydrograph and the lower aquifer in the bottom hydrograph. By limiting average withdrawal rates to about $2 \mathrm{mgd}$ and by utilizing surface-water recharge, drawdowns have been kept to about 2 feet in the lower aquifer. At this rate of withdrawal there is very little depletion of storage. A special effort has been made to reduce the rate and amount of drawdowns. Water from West Fork Portage Creek and a connecting artificial channel are induced through the upper aquifer to maintain higher water levels and reduce pumping costs (Reed and others, 1966). For the period 1960-66, the well field appears to be in balance. That is, recharge balances withdrawal. The period of record of pumpage includes the drought years of 1963 and 1964, when recharge was generally deficient in Kalamazoo County. Thus, the available water for development is at least equal to the average withdrawal rate of $2.38 \mathrm{mgd}$ (table 15). Although the upper and lower aquifers are relatively thick and of high transmissibility, increased development probably should be limited to about $3 \mathrm{mgd}$ because of the proximity of pumping center $\mathrm{F}$ with its very large withdrawals. In order to monitor the effects of future changes in withdrawal, the two observation wells at Station 9 should be continued.

There are five well fields at pumping center F. About 98 percent of the water withdrawn at the pumping center is from wells of the Upjohn Co. As mentioned before, 60 to 80 percent of the Upjohn withdrawal, after use, is returned to artificial recharge basins, to Portage Creek, and since 1967, to Austin Lake. The quantities of water returned to the various places change from time to time depending on local needs. Therefore, comparisons of water-level fluctuations to show change in storage are influenced by recirculation and do not indicate potential yield. They do indicate if with- 


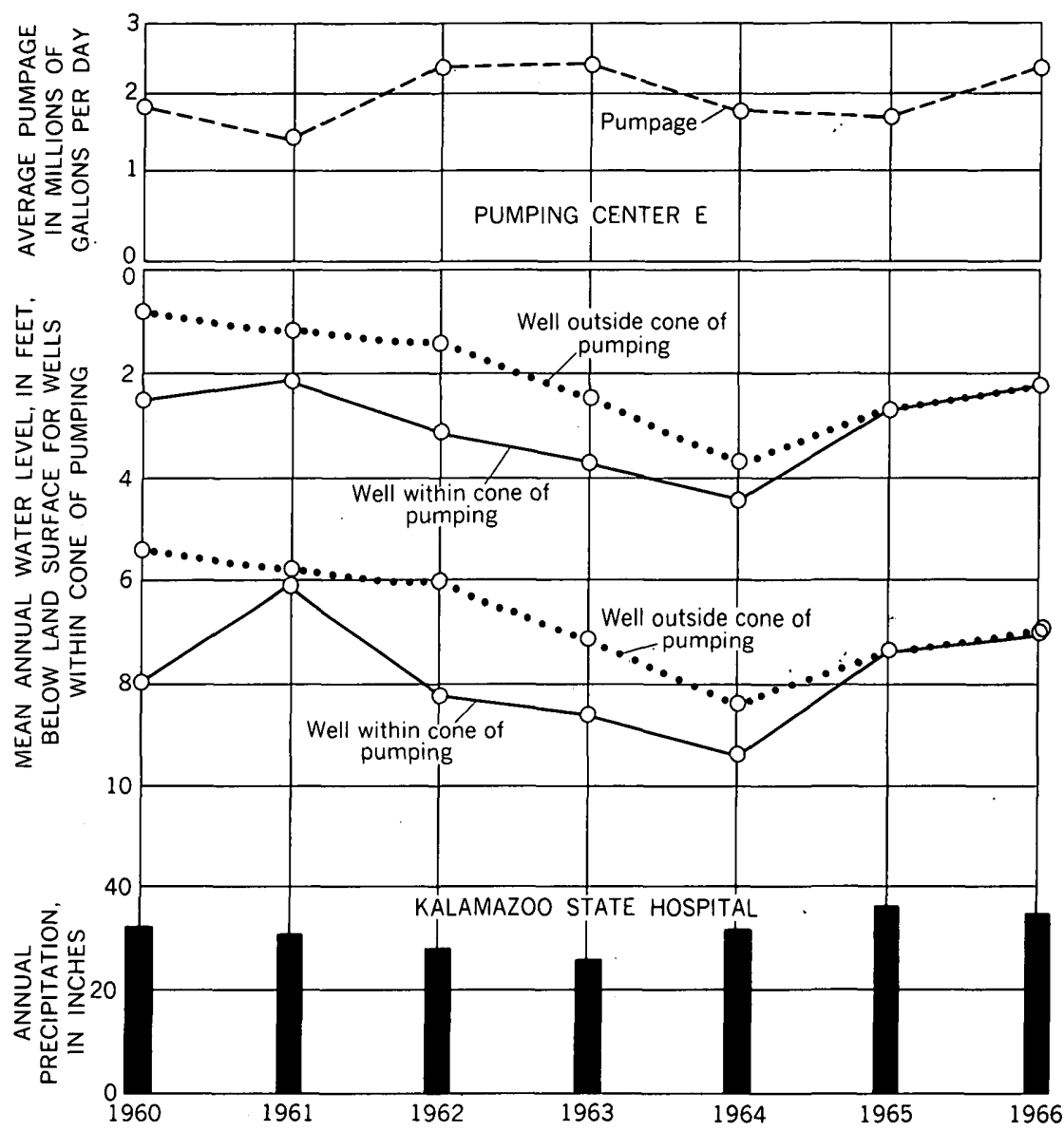

Figure 31.-Relationship between water-level fluctuations, pumpage, and precipitation at pumping center $\mathbf{E}$ of the Kalamazoo-Portage ground-water reservoir: $1960-66$.

drawals are affecting drawdowns and for this reason are shown in figure 32. The graphs from the wells outside the cone of depression show the same water-level data and are static-water levels from the same well. The graphs of the wells within the cone of pumping are pumping levels from representative wells at each of the Upjohn Co.'s central, north, and south well fields. The graphs showing static-water levels were matched with the graphs showing pumping levels to indicate minimum change in drawdown. All wells penetrate the lower aquifer. In the central well field, drawdown does not appear to be increased when average withdrawal rates equal about $1 \mathrm{mgd}$. In the north well field, drawdown apparently is not increased appreciably when average withdrawal rates do not exceed about 5.5 


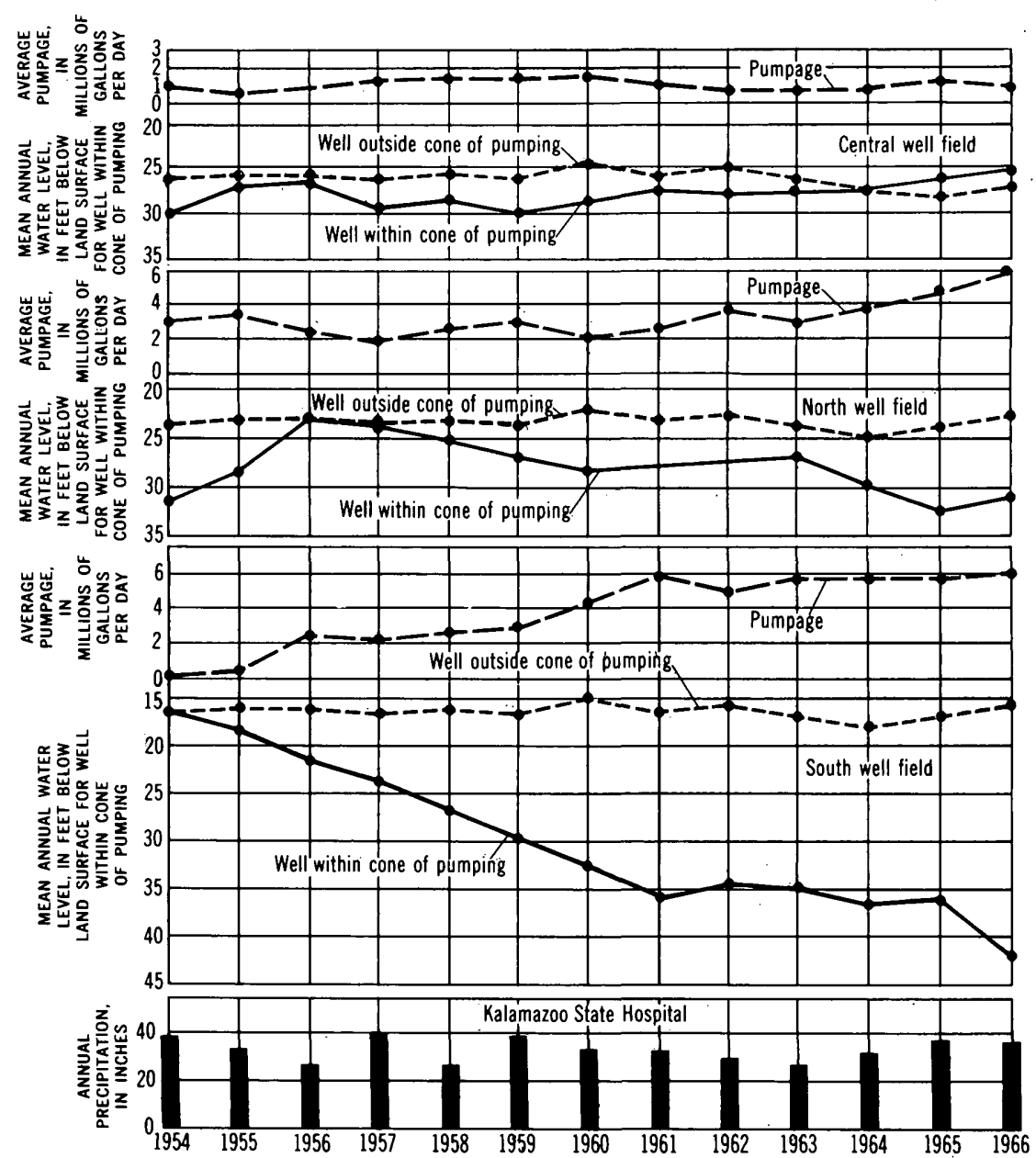

Frgure 32.-Relationship between water-level fluctuations, pumpage, and precipitation at three well fields of the Upjohn Co. for the period 1954-66. The well fields are located in pumping center $F$ of the Kalamazoo-Portage groundwater reservoir.

mgd. In the south well field, drawdown has increased, but withdrawal rates of about $5.5 \mathrm{mgd}$ are approximately balanced by recharge.

From an examination of pumpage from 1960 through 1966 at the five well fields in pumping center $F$ (table 15) and the graphs in figure 32, it appears that, taking into account present conditions of artificial recharge, average withdrawal rates from this pumping center should not be increased to more than about $13 \cdot \mathrm{mgd}$. With no artificial recharge, withdrawal rates would be limited to the recharge from precipitation. At a rate of 9 inches per year on an estimated recharge area of the center of 9 . sq $\mathrm{mi}$, this would amount 
to about $3.5 \mathrm{mgd}$. Therefore, the potential yield at pumping center $\mathbf{F}$ of about $13 \mathrm{mgd}$ includes about $9.5 \mathrm{mgd}$ recirculated by artificial recharge. In order to monitor the changing effects of withdrawals and recharge, the collection of records of water-level fluctuation and pumpage in the vicinity of the Upjohn Co.'s well fields should be continued.

An estimated potential yield for the existing centers in the Kalamazoo-Portage ground-water reservoir is summarized in the following table (data in million gallons per day):

\begin{tabular}{|c|c|c|c|}
\hline Pumping center & $\begin{array}{c}\text { Average dally } \\
\text { pumping in } \\
\text { 1966 }\end{array}$ & $\begin{array}{l}\text { Potentilal } \\
\text { Increase In } \\
\text { pumpage }\end{array}$ & $\begin{array}{c}\text { Practical } \\
\text { limlt of } \\
\text { development }\end{array}$ \\
\hline $\begin{array}{l}\text { A } \\
\text { B } \\
\text { B } \\
\text { C } \\
\text { D } \\
\text { E } \\
\text { F }\end{array}$ & $\begin{array}{r}2.41 \\
.77 \\
.85 \\
1.78 \\
2.38 \\
12.77\end{array}$ & $\begin{array}{r}0.59 \\
.23 \\
.15 \\
1.22 \\
.62 \\
.23\end{array}$ & $\begin{array}{r}\text { 3. } 00 \\
\text { 1. } 00 \\
\text { 1. } 00 \\
\text { 3. } 00 \\
\text { 3. } 00 \\
\text { *13. } 00\end{array}$ \\
\hline Total. & 20. 96 & 3. 04 & $* 24.00$ \\
\hline
\end{tabular}

-Includes about $9.5 \mathrm{mgd}$ withdrawn and recirculated by artificlal recharge.

No great accuracy is claimed for the estimates of potential yield of the pumping centers. However, unless additional provisions are made for artificial recharge, it is believed that the KalamazooPortage ground-water reservoir should not be developed to a greater degree than the average rate of about $24 \mathrm{mgd}$. Additional artificial recharge can be accomplished by diverting storm runoff into "dry" (above the water table) recharge basins in the vicinity of pumping centers $\mathrm{A}, \mathrm{C}$, and $\mathrm{D}$. Induced stream infiltration is already practiced, and some recharge ponds are in operation at centers $\mathrm{D}, \mathrm{E}$, and F. It does not appear desirable to develop additional pumping centers in this ground-water reservoir. The practical level of development can be reached by new well fields and artificial recharge at existing pumping centers.

\section{GHEMICAL QUALITY}

Although large amounts of water have been withdrawn from the Kalamazoo-Portage ground-water reservoir, representative chemical characteristics such as dissolved solids, hardness, sulfate, and chloride are lower than those for the heavily pumped Kalamazoo River reservoir. Induced recharge has decreased the concentration of dissolved solids of some of the water produced from the KalamazooPortage reservoir. Water from most surface sources has low concentrations of the major mineral constituents. The graphs for the 
pumping centers, (pl. 8), indicate that the ground water is very hard and that the iron content is higher then the recommended limits. Although the water in some of the streams and in the groundwater reservoir is somewhat high in dissolved solids, iron, and hardness, the water could easily be treated by common methods to produce water of excellent quality. At pumping center $F$, the city of Portage Site $\mathrm{C}$ well field was abandoned because of the high iron content in the water. At the Station 22 well field of the city of Kalamazoo in pumping center D, water from West Fork Portage Creek indirectly recharges the lower aquifer. Water from the Creek is low in dissolved-solids content (pl. 6) and has not adversely affected the quality of water in the lower aquifer. Water induced into the lower aquifer from an artificial recharge channel and pond connected to West Fork Portage Creek, at the Station 9 well field of the city of Kalamazoo in pumping center $\mathrm{E}$, has caused no significant increase in concentration of major mineral constituents (Reed and others, 1966, table 5).

In 1960, three new wells in the vicinity of the Upjohn Co.'s southeast recharge pond at pumping center $F$ were put into service. In an old well, No. 5, about 1,400 feet from the three new wells, there was a sharp rise in temperature, dissolved solids, hardness, and chloride (fig. 33). Apparently the rise is the result of inducing recharge water of higher temperature and mineral content from the recharge pond into the lower aquifer. The effects of induced recharge can be seen at the south well field in figure 32. From 1961 through 1965, pumpage balanced induced recharge and pumping water levels remained at about 35 feet below land surface. In 1966, the pumping rates of two nearby wells were increased and the pumping water levels declined to about 42 feet.

Pumping centers adjacent to streams crossing the KalamazooPortage reservoir could induce recharge water with high concentrations of dissolved solids into the reservoir. Allen Creek (pl. 6) and the lower reach of Portage Creek near Kalamazoo (fig. 34) are relatively high in dissolved-solids content. Well fields at pumping center $\mathrm{B}$ and $\mathrm{E}$ near Portage Creek and at pumping center $\mathrm{C}$ near Allen Creek have not shown any significant increase in chemical content, probably because they are not heavily pumped (pl. 8).

\section{OTHER GROUND-WATER RESERVOIRS}

In addition to the three large ground-water reservoirs already discussed, there are seven smaller reservoirs and another isolated well field, operated by the city of Kalamazoo as Station 19, from which large supplies of ground water can be withdrawn. Generally 


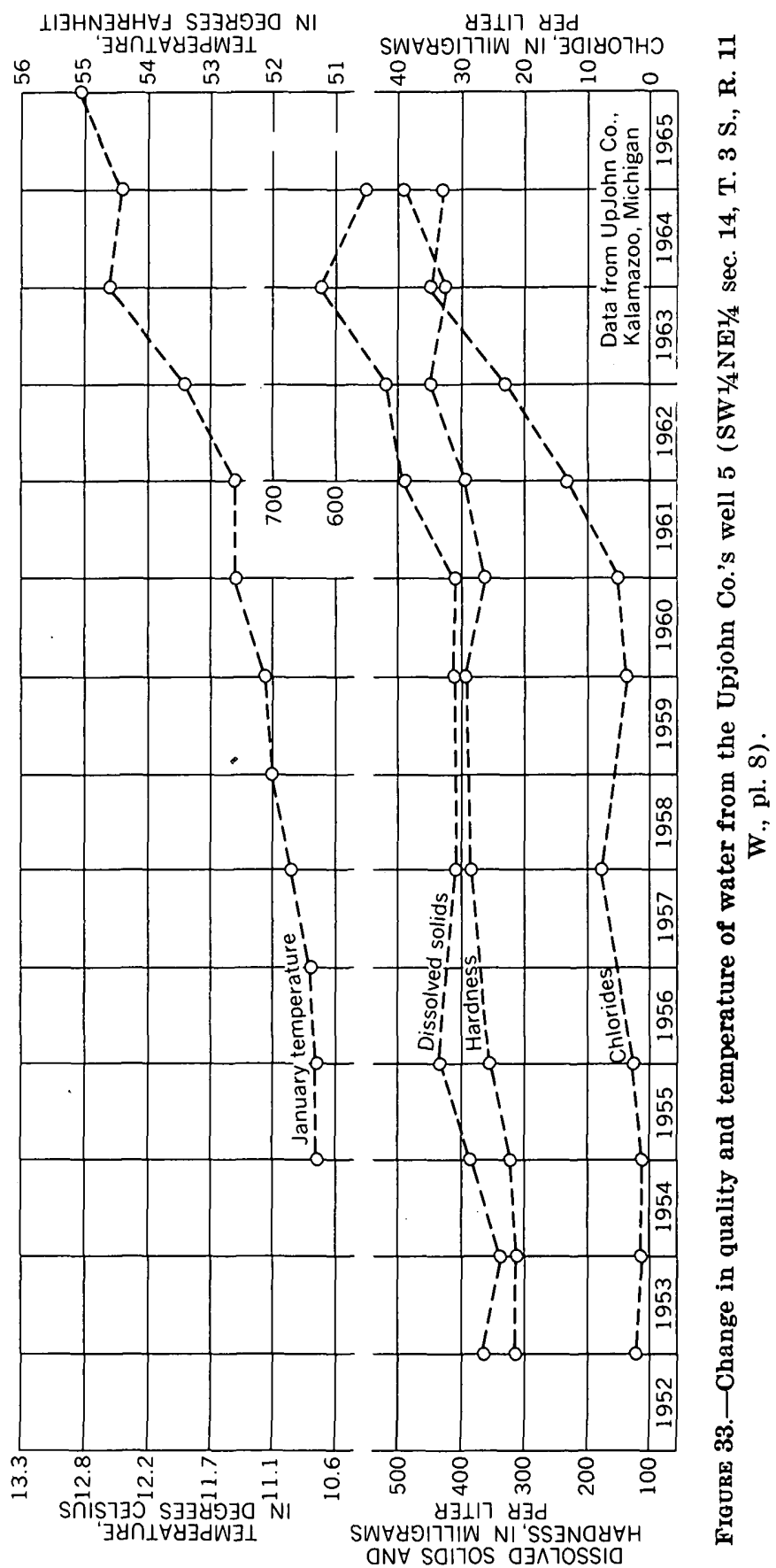




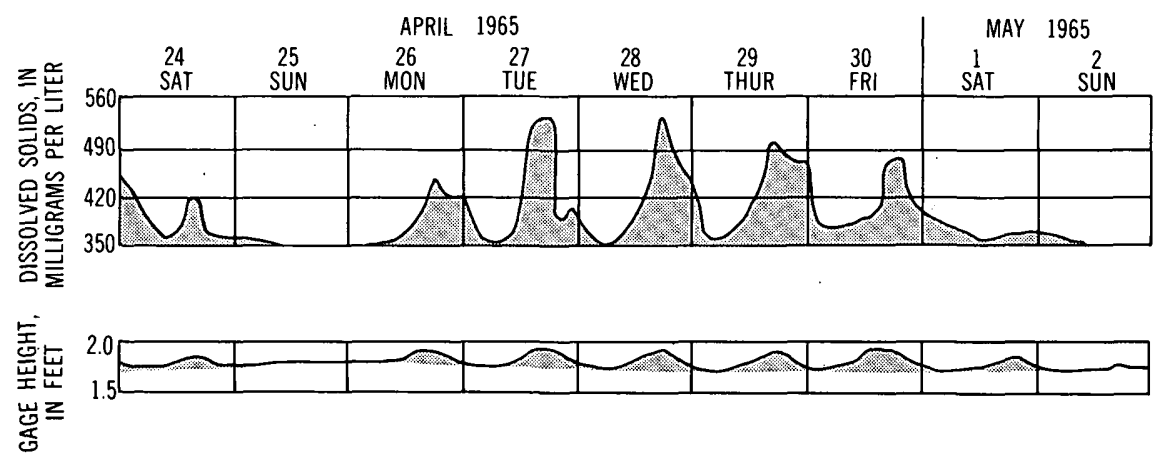

Figure 34.-Dissolved solids and gage height as affected by industrial waste disposal in Portage Creek near Kalamazoo.

named for their geographic location, they are Cooper, Morrow Lake, Augusta-Galesburg, Alamo-Oshtemo, and Sherman Lake in the upper aquifer; and Vicksburg, Texas, and the isolated well field in the lower aquifer. They are characterized by areas of greater-thanaverage saturated thickness and relatively high coefficients of transmissibility. Their locations are shown by rectangles on plates 2, 4, and 8 . The isolated well field is shown on plate 8. Other areas may have either greater saturated thickness or higher transmissibility, but because they do not have both characteristics, they are not considered ground-water reservoirs for the purpose of this report. The physical and hydraulic characteristics and the idealized scheme of development of these ground-water reservoirs are shown by mathematical models (pl. 9) and are discussed in following sections of the report. For each of the reservoirs, the average recharge from precipitation was estimated to be 9 inches per year. The chemical quality of water in the reservoirs is generally good; however, it is hard and locally contains objectionable amounts of iron.

An observation well should be installed in each ground-water reservoir and in the isolated well field to monitor future changes in water-level fluctuations as these reservoirs and the well field are developed.

\section{COOPER GROUND-WATER RESERVOIR}

The Cooper ground-water reservoir underlies a part of Cooper Township that is adjacent to the Kalamazoo River. It joins the Kalamazoo River ground-water reservoir on the south. At present (1968) there are no withdrawals from this upper aquifer reservoir. The mathematical model assumes water-table conditions and a pumping rate of about $6 \mathrm{mgd}$ for 180 days without recharge from eight wells, each pumping at $500 \mathrm{gpm}$. Pumping would lower water levels a maximum of 26 feet at the pumping wells, and the effect would 
be negligible beyond 5,000 feet. Recharge from precipitation on a 30 -sq mi area, including about $25 \mathrm{sq}$ mi outside the reservoir, would be about $13 \mathrm{mgd}$. Stream recharge from Spring Brook is included in the estimate of recharge from precipitation. Based on the infiltration capacity of streams overlying the reservoir (p. 85), recharge of streamflow from the Kalamazoo River and Spring Brook could be at an average rate of about $7 \mathrm{mgd}$. Thus, although the model assumes a pumpng rate of only $6 \mathrm{mgd}$, with stream infiltration the potential yield would be $13 \mathrm{mgd}$. More streamflow than the $7 \mathrm{mgd}$ could be induced into the ground-water reservoir artificially. However, the bottom and sides of the Kalamazoo River appear to be partially sealed, which will limit the amount of recharge from streamflow. Also, water in the river has a high dissolved-solids content so that during dry periods, when streamflow is low, it may not be desirable to infiltrate as much as $7 \mathrm{mgd}$. At such times water from storage in the aquifer or water infiltrated from Spring Brook could be used in place of induced recharge from the Kalamazoo River. Thus, a potential yield of $13 \mathrm{mgd}$ is felt to be a conservative approach to the practical level of development in the Cooper groundwater reservoir. Part of the recharge from precipitation and part of the induced stream recharge would be utilized. Artificial recharge facilities along Spring Brook could utilize flood flows and increase the potential development.

The Cooper ground-water reservoir is undeveloped and, therefore, has not been affected by contaminated water from Kalamazoo River. If the entire reservoir were developed, the concentration of most chemical constituents would be increased in the reservoir because water of poor quality from the Kalamazoo River would infiltrate. However, if the southern part of the reservoir adjacent to Spring Brook were developed, water of good chemical quality from Spring Brook could be induced into the reservoir. Thus, for future practical development, both the chemical composition and the concentration of the contaminating substances should be considered.

\section{MORROW LAKE GROUND-WATER RESERVOIR}

The Morrow Lake ground-water reservoir in the upper aquifer underlies an area of Comstock Township where the Kalamazoo River has been dammed to form Morrow Lake. The model of the Morrow Lake ground-water reservoir is similar to that for the Kalamazoo River and Cooper ground-water reservoirs in that it assumes water-table conditions. The assumed pumping rate is about $3 \mathrm{mgd}$ for 180 days without recharge from five wells pumping at $400 \mathrm{gpm}$ each. Pumping would lower water levels a maximum of 22 feet at 
the pumping wells. Drawdown would be negligible beyond 5,000 feet. Recharge from precipitation on a 24-sq-mi area, including an area of $22 \mathrm{sq} \mathrm{mi}$ outside the reservoir, would be about $10 \mathrm{mgd}$. Based on infiltration capacity (p. 85), an additional $7 \mathrm{mgd}$ of induced infiltration from Morrow Lake could provide for replenishment of withdrawals. Because of silting of the bottom and sides of Morrow Lake; most of the infiltration would occur at times of high lake stage: Development of $10 \mathrm{mgd}$ is believed to be a conservative approach to the potential yield of the reservoir, where large amounts of streamflow from the upstream reach of the Kalamazoo River are available for infiltration. The magnitude of withdrawals contemplated should have little effect on the supply of surface water in Morrow Lake because most of the infiltration to the reservoir would occur at a time when lake levels were at high stages relative to ground-water levels. At the present time there are no apperciable withdrawals from the Morrow Lake ground-water reservoir.

Future development of the Morrow Lake ground-water reservoir will induce water from Morrow Lake. The present chemical quality of the lake water is relatively good; however, with increased use of the Kalamazoo River upstream, the possibility of chemical contamination must be considered. Bacterial contaminents would be largely filtered out when surface water moves into the ground-water reservoir.

AUGUSTA-GALESBURG GROUND-WATER RESERVOIR

The Augusta-Galesburg ground-water reservoir in the upper aquifer underlies the Kalamazoo River valley in a reach extending roughly from Augusta to Galesburg. It joins the Morrow Lake ground-water reservoir to the west and the Sherman Lake groundwater reservoir to the north. The model of the reservoir is similar to that of the Kalamazoo River, Cooper, and Morrow Lake groundwater reservoirs in that it assumes water-table conditions. The model assumes a pumping rate of $400 \mathrm{gpm}$ from 14 wells, or a total rate of $8 \mathrm{mgd}$ for 180 days without recharge. Pumping would result in maximum drawdowns of 32 feet at the supply wells. Drawdown would be negligible beyond 6,000 feet.

In determining the potential recharge of the Augusta-Galesburg ground-water reservoir, it is assumed that recharge available to the Sherman Lake ground-water reservoir is not available to the $\mathrm{Au}$ gusta-Galesburg reservoir. Thus, potential recharge from precipitation on the area is somewhat reduced, and potential induced recharge from Gull Creek is small: Potential induced recharge from Augusta Creek and Kalamazoo River is not affected by development of the Sherman Lake reservoir and is large. It is estimated that recharge 
from precipitation could be at a rate of $14 \mathrm{mgd}$ from a 32 -sq-mi area, including an area of $20 \mathrm{sq} \mathrm{mi}$ outside the reservoir. Assuming that the reaches of Kalamazoo River, Augusta Creek, and Gull Creek overlying the reservoir total about 30,000 feet and that the streaminfiltration rate is 400,000 gpd per 1,000 feet (p. 84), the total recharge from stream infiltration would be about $12 \mathrm{mgd}$. Thus, by utilizing recharge from precipitation and stream infiltration, the total potential yield of the Augusta-Galesburg ground-water reservoir is estimated to be about $26 \mathrm{mgd}$.

Present withdrawals from the Augusta-Galesburg. ground-water reservoir are limited to well fields at the village of Augusta and the city of Galesburg. In 1966 pumpage from the two well fields averaged $0.16 \mathrm{mgd}$. The combined pumpage for the two well fields from 1960 to 1966 shows an increase of $0.03 \mathrm{mgd}$ (table 15). As indicated above, the potential yield of the reservoir is conservatively estimated to be $26 \mathrm{mgd}$ and the reservoir is undeveloped. The small amount of development that has taken place has not adversely affected the chemical quality of the water. Further development probably will induce water from the Kalamazoo River and Gull and Augusta Creeks into the reservoir. The chemical quality of the Kalamazoo River in this reach is generally good; however, a better quality water that is less subject to contamination is found in Gull and Augusta Creeks.

\section{ALAMO-OSHTEMO GROUND-WATER RESERVOIR}

The Alamo-Oshtemo ground-water reservoir in the upper aquifer underlies the southern part of Alamo Township and the northern part of Oshtemo Township. It is assumed that the reservoir is under water-table conditions. Using the model, maximum drawdowns from five wells, pumping at $400 \mathrm{gpm}$ or about $3 \mathrm{mgd}$ for 180 days without recharge, would be 24 feet at each well. Drawdowns would be negligible beyond 6,000 feet. Available drawdown is 70 feet, so that it would be possible to pump at greater pumping rates from a smaller number of wells to get a large drawdown. It was estimated that recharge from precipitation on a 6 -sq $\mathrm{mi}$ area, including $1 \mathrm{sq} \mathrm{mi}$ outside the reservoir, could support a withdrawal rate of about $3 \mathrm{mgd}$. As there are no streams from which recharge could be induced, withdrawals are limited to the recharge from precipitation and the assumed withdrawal rate of $3 \mathrm{mgd}$ is the practical limit of development. If wells are pumped at rates greater than about $3 \mathrm{mgd}$, water would be removed from storage and the reservoir would be dewatered. At this time (1968) there are no appreciable withdrawals from the Alamo-Oshtemo ground-water reservoir. The quality of 
water in the reservoir should remain relatively good provided the recharge water is also of good quality. The potential yield could be increased somewhat by capturing surface runoff in dry recharge basins.

\section{SHERMAN LAKE GROUND-WATER RESERVOIR}

The Sherman Lake ground-water reservoir in the upper aquifer underlies an area extending from Gull Lake on the north to Augusta on the south. It joins the Augusta-Galesburg ground-water reservoir on the south. With the selected scheme of development from the model under water-table conditions, maximum drawdowns, from six wells pumping at $750 \mathrm{gpm}$ or about $6 \mathrm{mgd}$ for 180 days without recharge, were 36 feet at the pumping wells. Drawdowns are negligible beyond 6,000 feet. It was estimated that recharge from precipitation on a 17-sq-mi area, including an area of $10 \mathrm{sq}$ mi outside the reservoir, could support a withdrawal rate of about $7 \mathrm{mgd}$. Because of the proximity of Gull Lake to the reservoir, it was estimated that withdrawal rates greater than $6 \mathrm{mgd}$ would affect the level of Gull Lake. Thus, recharge of $7 \mathrm{mgd}$ can restore assumed withdrawals; temporary deficiencies, if they existed, could come from storage for short periods. The assumed withdrawal rate of $6 \mathrm{mgd}$ is about the practical limit of development. Development of the reservoir would induce recharge from Gull Creek, but this water has already been included in estimates of recharge from precipitation and thus is not available to recharge the reservoir. Additional benefits in operating future well fields could be obtained by constructing artificial channels and recharge ponds along Gull Creek to induce streamflow into the ground-water reservoir. The advantages would be to reduce drawdowns and to increase recharge of good quality water. The selected scheme of development of $6 \mathrm{mgd}$ is a conservative approach to the potential yield of the reservoir. At present (1968), there are no appreciable withdrawals from the reservoir. As mentioned previously, the problems of disposal of domestic waste in the Gull Lake area should be studied. The waste is a possible source of contamination to the reservoir.

\section{VICKSBURG GROUND-WATER RESERVOIR}

The Vicksburg ground-water reservoir underlies the village of Vicksburg and the surrounding area. It joins the Schoolcraft groundwater reservoir on the west. The upper and lower aquifers are separated by a leaky aquiclude. The average thickness of the upper aquifer is 20 feet; the lower aquifer, 40 feet; and the aquiclude, 40 feet. Average coefficients of transmissibility and storage of the upper aquifer are estimated to be $30,000 \mathrm{gpd}$ per foot and 0.20 , and 
of the lower aquifer, about $40,000 \mathrm{gpd}$ per foot and 0.005 , respectively. Using the idealized scheme of development in the model, six supply wells pumping at $500 \mathrm{gpm}$ or about $4 \mathrm{mgd}$ for 180 days without recharge would have a maximum drawdown of 21 feet at the supply wells. Drawdowns are negligible beyond 6,000 feet. It was estimated that an average annual recharge from precipitation of $4 \mathrm{mgd}$ reaches the upper aquifer from a recharge area of about $10 \mathrm{sq} \mathrm{mi}$, including a 5-sq-mi area outside the reservoir. Reservoir storage of the upper aquifer could provide an estimated withdrawal rate of $4 \mathrm{mgd}$ for 180 days without recharge. Potential leakage through the aquiclude is about $4 \mathrm{mgd}$. Thus, the assumed pumping rate of $4 \mathrm{mgd}$ from the lower aquifer is the practical limit of development from natural recharge. Based on the seepage run of July 27 and 28, 1964-a time when the streamflow was very lowit is estimated that about $3 \mathrm{cfs}(2 \mathrm{mgd})$ of potential stream recharge is available to the reservoir from Gourdneck Creek (pl. 4). Utilizing total recharge of $6 \mathrm{mgd}$ from the above sources would have small effect on lake stage at Sunset and Barton Lakes and streamflow in Portage Creek. It should be noted that, below Sunset Lake, Gourdneck Creek becomes Portage Creek. The degree of effect would depend upon the amount of water recirculated back to the ground-water reservoir or to Portage Creek.

At present (1968) the Vicksburg ground-water reservoir is relatively undeveloped. Pumpage from well fields of the village of Vicksburg and the Lee Paper Co. has increased from 1.7 to $2.0 \mathrm{mgd}$ in the period 1960 through 1966 (table 15). Much of the water withdrawn is recirculated back to the reservoir or to Portage Creek. The potential yield of $6 \mathrm{mgd}$ is about triple the current average withdrawal rate of $2.0 \mathrm{mgd}$.

Future development of the reservoir should consider inducing water from Gourdneck Creek and Sunset Lake above the village of Vicksburg. Below Vicksburg, Portage Creek and Barton Lake are degraded by industrial and municipal disposal and are generally, because of quality considerations, not as well suited for induced recharge

\section{TEXAS GROUND-WATER RESERVOIR}

The Texas ground-water reservoir underlies the central part of Texas Township and joins the Kalamazoo-Portage ground-water reservoir on the east. It consists of an upper and lower aquifer separated by a leaky aquiclude. The upper aquifer has an average thickness of 80 feet; the lower aquifer, 50 feet; and the aquiclude, 100 feet. Average coefficients of transmissibility and storage of the upper aquifer are estimated to be $60,000 \mathrm{gpd}$ per foot and 0.20 , and 
of the lower aquifer, about $100,000 \mathrm{gpd}$ per foot and 0.005 , respectively. Using the idealized scheme of development in the model, five supply wells pumping at $400 \mathrm{gpm}$ or $3 \mathrm{mgd}$ for 180 days without recharge from the lower aquifer would have a maximum drawdown of 29 feet at the supply wells. Drawdowns are negligible beyond 20,000 feet. Available drawdown is estimated to be 180 feet, so that it would be possible to withdraw water with a smaller number of wells at a higher rate of pumping than in the model. It was estimated that an average annual recharge from precipitation of $3 \mathrm{mgd}$ reaches the upper aquifer from a recharge area of $7 \mathrm{sq} \mathrm{mi}$, including a 2-sq-mi area outside the reservoir. Reservoir storage of the upper aquifer could provide an estimated withdrawal rate of $3 \mathrm{mgd}$ for 180 days without recharge. Potential leakage through the aquiclude of about $3 \mathrm{mgd}$ is adequate to support the assumed pumpage. Thus the assumed pumping rate of $3 \mathrm{mgd}$ is the practical limit of development from recharge. Induced recharge is available from West Fork Portage Creek only to maintain higher water levels in the upper aquifer and reduce pumping costs. It is a part of the total recharge and cannot be used to supplement the practical yield. Using the above scheme of development, there would be some effect on lake levels at Eagle, Bass, and Crooked Lakes and on low flow from West Fork Portage Creek. The degree of effect would depend upon the amount of water recirculated back to the lakes or creek.

Water from the Texas ground-water reservoir has been pumped into Crooked and Eagle Lakes and seeps back into the ground-water reservoir. In 1966, an average of $2.52 \mathrm{mgd}$ was pumped into the two lakes (table 15). The effects of this pumping on lake levels is shown in figure 21. Because withdrawals are intermittent and the water is recirculated, withdrawals have had little permanent effect on storage in the ground-water reservoir. Future development in the reservoir should consider inducing surface water of good quality from West Fork Portage Creek.

\section{ISOLATED WELL FIELD}

Another well field of the city of Kalamazoo, Station 19, pumps water from the lower aquifer in a northwest extension of the Kalamazoo-Portage ground-water reservoir (pl. 8). There is no upper aquifer. Based on available geologic and hydrologic data in the vicinity of the well field, the average saturated thickness of the lower aquifer is 40 feet and the average coefficient of transmissibility is $20,000 \mathrm{gpd}$ per foot (pls. 1, 2). None of the wells withdraw large amounts of water. At one well, the water level under static conditions was reported to be about 2 feet below land surface and the drawdown was 36 feet at a pumping rate of $400 \mathrm{gpm}$. Average 
annual pumpage from 1962 through 1966 ranged from $0.19 \mathrm{mgd}$ in 1962 to a maximum of $0.71 \mathrm{mgd}$ in 1964 (table 15). The practical limit of development is probably small and about equal to the withdrawal rate of about $0.71 \mathrm{mgd}$ recorded in 1964, a drought year.

\section{SUMMARY OF POTENTIAL DEVELOPMENT}

The practical potential development of the ground-water reservoirs in Kalamazoo County is summarized in the following table (data in million gallons per day):

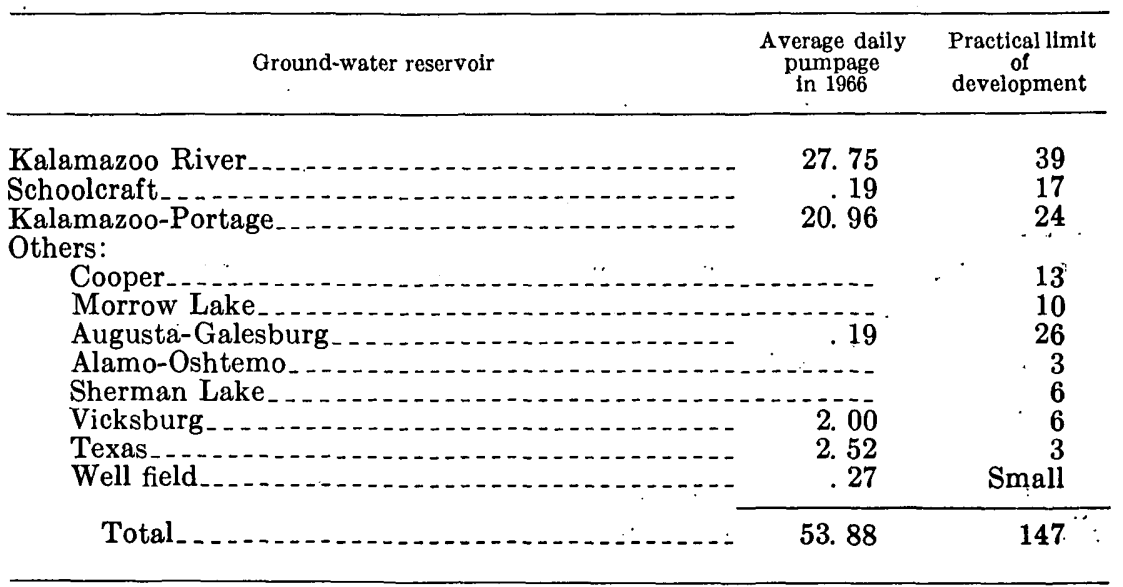

The practical limit of development of the ground-water reservoirs is considerably larger than present pumpage. The table also shows the reservoirs in which the greatest development can take place. The potential development is called practical because the rate indicated will have a minimum effect on surface-water supplies and minimum interference between pumping centers and ground-water reservoirs. Estimates of potential development are conservative and substantially larger quantities are available, particularly if induced recharge of surface waters is fully utilized and other types of artificial recharge are practiced.

Large-scale development of the ground-water reservoirs will produce a great deal of new data. Any large-scale development should be carefully observed and the data critically analyzed. It will almost certainly be desirable to adjust the pattern of development to take advantage of the new data and make optimum use of the groundwater reservoirs.

The evaluation of the ground-water reservoirs can also serve as a basis to permit the application of the electric analog model or digital computer to the analysis of current and future water-resource problems of the area. 


\section{NONRESERVOIR AREAS}

Areas outside of the designated ground-water reservoirs are referred to as nonreservoir areas. Large parts of the nonreservoir areas are underlain by aquifers of sufficient thickness, transmissibility, and areal extent to be shown on plates 1 and 2. The remainder of the nonreservoir areas consist of aquicludes or aquifers that are too thin or of too limited areal extent to be shown. The productivity of wells in the nonreservoir areas varies considerably. Where the aquifers are of sufficient thickness to be mappable, wells commonly will yield moderate supplies, generally enough for small industries. In the areas where aquifers are not shown, generally only small supplies, enough for domestic needs, can be obtained. In a few localities, however, it is possible that large supplies may be obtained even in the areas where aquifers are not shown to be present on plates 1 and 2.

Development of the nonreservoir areas has been small. A few industries have wells, and rural residents and farm operators are supplied from individual wells, as are the residents of some small communities in the area.

Most nonreservoir areas of the county are capable of yielding water of good quality for domestic, stock, and light industrial uses. The quality of this water is similar to that produced from the major ground-water reservoirs. It is a calcium bicarbonate type of water that is very hard and often contains objectionable quantities of iron. However, water-conditioning equipment that can remove iron and hardness at moderately low cost is readily available. Figure 35, which illustrates the range of concentration of selected ions based on 280 analyses, indicates that about 90 percent of domestic supplies have very hard water (generally more than $180 \mathrm{mg} / \mathrm{l}$ ). The median chloride concentration is $10.2 \mathrm{mg} / \mathrm{l}$, and the range is from 1 to 60 $\mathrm{mg} / \mathrm{l}$. The median value of dissolved solids is $365 \mathrm{mg} / \mathrm{l}$, and the range is from 240 to $620 \mathrm{mg} / 1$. Analyses of municipal supplies which are unaffected by induced recharge indicate that sulfate has an average value of 29 and ranges from 2 to $54 \mathrm{mg} / \mathrm{l}$.

Chloride and sulfate cannot be economically removed from domestic water sources, but their concentrations are relatively low and do not create a problem. Hardness can be reduced easily and economically in individual domestic supplies by ion exchange methods. The use of modern detergents reduces the problem of water hardness. Some people consider the very hard water of the county to be satisfactory for household use without treatment.

U.S. Public Health Service (1962) recommends that maximum iron content should not be more than $0.3 \mathrm{mg} / \mathrm{l}$. This is not a toxic 

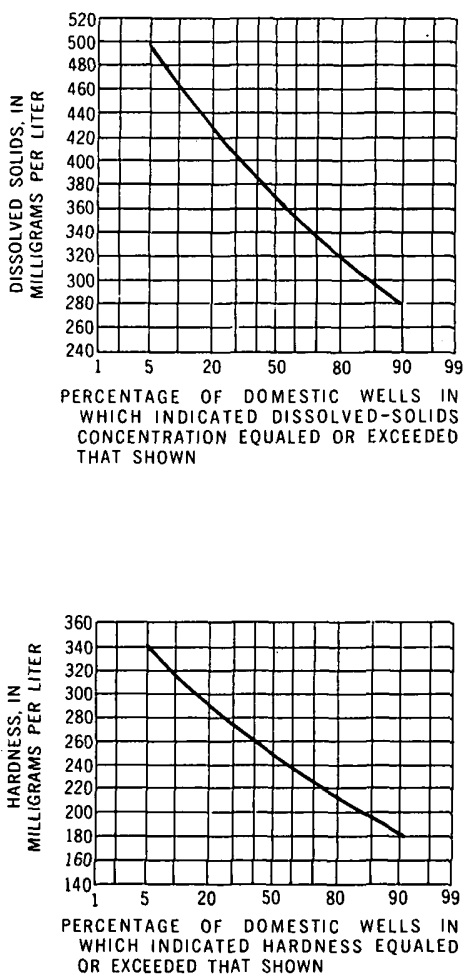
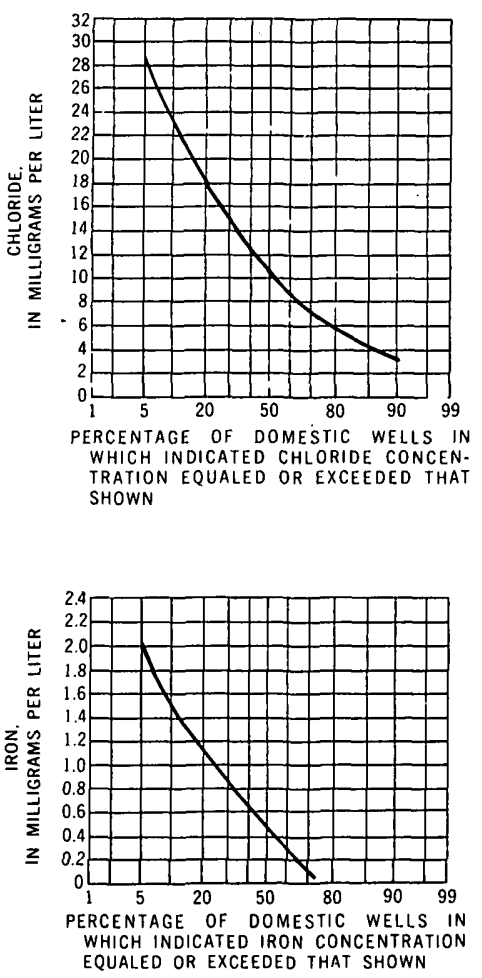

Froure 35.-Chemical quality of water in about 280 domestic wells.

limitation but one above which staining of porcelain fixtures and laundered articles can occur. The median iron concentration in eight analyses was $0.4 \mathrm{mg} / \mathrm{l}$ and ranged from 0 to $4.2 \mathrm{mg} / \mathrm{l}$ (fig. 35 ).

For the individual domestic water supply, water treatment is the only practical answer for objectionable iron concentration. Selection of the proper type of commercial treatment method depends upon the use of the water and the original iron content. In general, the methods fall into categories of either stabilization or ion exchange. Water-conditioning companies should be consulted for specific situations.

A thin bed of sandstone, which is present only in the northeastern part of Kalamazoo County, yields water of chemical quality similar to that obtained in the overlying unconsolidated deposits. A bed of shale underlying this sandstone contains saline water in neighboring Branch County (Giroux and others, 1966). Several wells finished in the upper part of the shale in the southeastern part of Kalamazoo County yield water which shows no significant difference in chemical quality from that obtained from wells tapping the unconsolidated 
aquifers. The chemical quality of the water in the shale in other parts of Kalamazoo County probably is inferior to that in the unconsolidated aquifers.

\section{GROUND-WATER WITHDRAWALS}

Kalamazoo County, where the ground-water withdrawal in 1966 was about 20 billion gallons (about $55 \mathrm{mgd}$ ), has the largest concentration of ground-water pumpage in Michigan and one of the largest in the Midwest. The population and industrial production of the county are certain to increase during the next few decades, and water demands will increase concurrently. The present distribution and amount of withdrawal are important considerations in an evaluation of the ground-water potential development of the county.

Ground-water withdrawals have increased by about $10 \mathrm{mgd}$ in the period from 1960 through 1966 . The average ground-water pumpage from the pumping centers and the ground-water reservoirs for this period is listed in table 15. From this table it is apparent that the distribution of withdrawal is changing. Average rates of pumping from the Kalamazoo River ground-water reservoir are decreasing; those from the Kalamazoo-Portage reservoir are increasing. Figure 36 illustrates the 1966 rates of withdrawal and available supply for the three principal ground-water reservoirs and a summary of the minor reservoirs. As indicated, about one-third of the potential supply is being withdrawn.

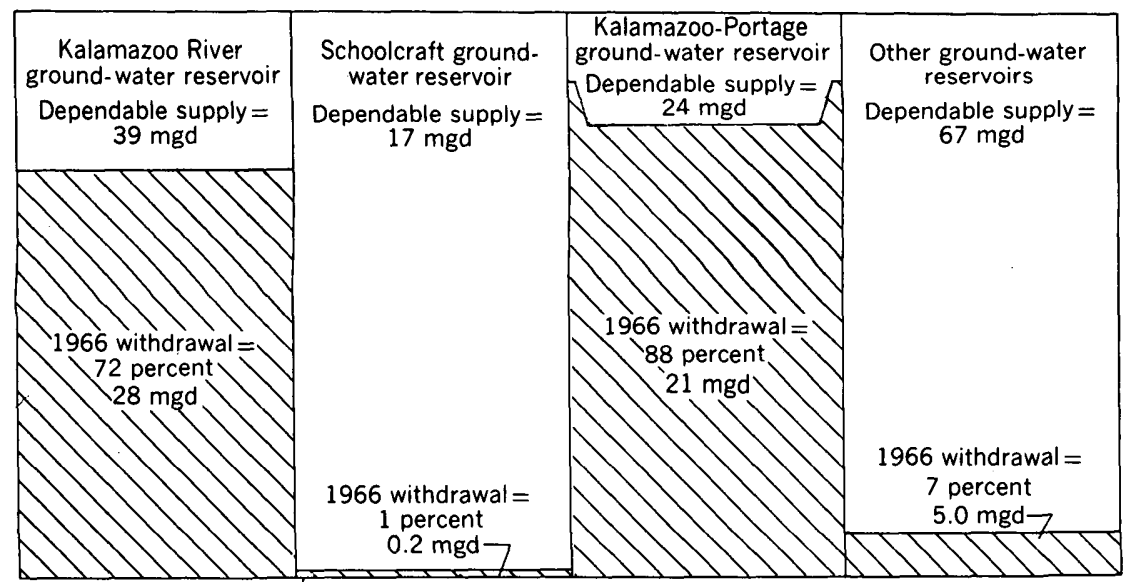

FTGure 36.-Comparison of ground-water withdrawal in 1966 with dependable supply. 
TABLE 15.-Average ground-water pumpage in million gallons per day from groundwater reservoirs and pumping centers, 1960-66

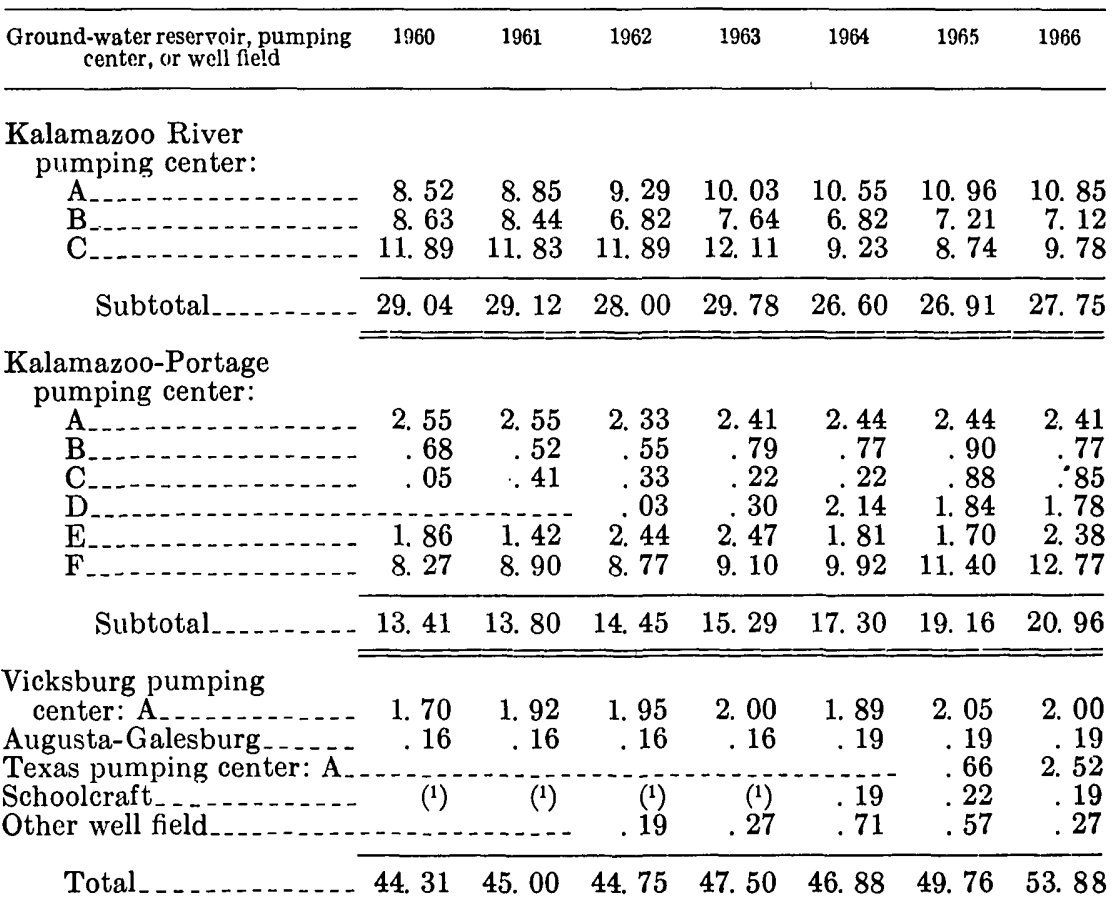

${ }^{1}$ Average pumpage less than $0.02 \mathrm{mg}$.

\section{LEGAL CONSIDERATIONS}

There are many acts prescribing water laws for the State of Michigan. These acts have recently been compiled in a publication prepared by the Joint Committee on Water Resource Planning of the Legislative Service Bureau (1966). The acts and statutes are too numerous to cover in this report, but a few of the more significant ones are mentioned below.

Under Act 245, Public Acts of 1929, as amended by Act 117, Public Acts of 1949; Act 165, Public Acts of 1963; and Act 405, Public Acts of 1965, the Michigan Water Resources Commission has the responsibility for protecting and conserving the water resources of the State and for control over the pollution of the waters of the State and the Great Lakes, with the power to make rules and regulations governing the same. The commission acts as the State agency to cooperate and negotiate with other governments and agencies in matters concerning the water resources of the State. 
Any person or organization requiring new or additional use of the waters of the State for waste-disposal purposes is required to file a written statement with the commission. The statement must include the amount of water required, its source, the point of discharge, and the physical and chemical characteristics of the wastes to be discharged.

Act 184, Public Acts of 1963, requires approval by the Michigan Department of Conservation for erection of dams in streams or rivers. No person shall construct or permit construction of any dam on land owned by him in any stream or river impounding more than 5 acre-feet without first obtaining a permit approving the plans for such construction.

Act 20, Public Acts of 1964, cited as the "surplus water act of 1964," regulates the impoundment and utilization of surplus water and prescribes the powers and duties of the Water Resources Commission and the several boards of supervisors. Any board or group of boards or local units may request the commission to undertake a survey of the water in a river basin located or partially located in a county or counties to determine whether there is surplus water available and if so, how it may be impounded, used, and conserved. Studies, surveys, and reports made by competent authorities may be used by the commission in making such determinations.

Act 146, Public Acts of 1961 (as amended by Acts 25 and 203, Public Acts of 1962), prescribes the duties and powers of the Department of Conservation and the boards of supervisors to determine and maintain the normal level of the waters in inland lakes, authorizes the building and maintenance of dams and embankments to accomplish such purposes, and prescribes procedures for financing and administering the facilities needed to accomplish these purposes.

Act 345, Public Acts of 1966, cited as the "inland lake improvement act of 1966," prescribes the powers and duties of the legislative bodies of local units of government and the Department of Conservation in providing for the improvement of certain inland lakes. It authorizes the dredging and removal of undesirable materials from lakes and prescribes the ways and means of financing such projects.

Act 291, Public Acts of 1965, cited as the "inland lakes and streams act" protects the riparian rights and the public trust in navigable inland lakes and streams.

Act 294, Public Acts of 1965, protects the public health, regulates the drilling of water wells and the installation of well pumps, registers and regulates water-well drillers and well-pump installers, and requires drillers to provide drilling records to the Department of 
Conservation, and prescribes the duties and powers of the State Health Commissioner.

Act 253, Public Acts of 1964, enables local units of government to cooperate in planning and carrying out a coordinated water management program in the watershed which they share.

Act 288, Public Acts of 1967, regulates the subdivision of land, requires that land be suitable for building sites and public improvements and that there be adequate drainage thereof. This act also establishes procedures relative to plots and controls residential building development within flood plains.

In general, the legal right to use surface water in Michigan is governed by the riparian doctrine. This doctrine states that a riparian owner (one who has property abutting a lake or stream) is entitled to have water undiminished in quantity and unimpaired in quality as the water passes his property.

In regard to ground water, the common law entitles a property owner to all water under his property. Under strict interpretation of the common law, a property owner could withdraw as much water from the ground as he desired without regard to the effects of such withdrawals on his neighbors. The courts, however, have applied a "reasonable use" restriction to the use of ground water. Reasonable use is subject to many interpretations, and in some cases may restrict withdrawals that have an adverse effect on others.

\section{SUMMARY AND CONCLUSIONS}

The availability and development of water in Kalamazoo County is summarized in table 16 and figures 18 and 36. The total dependable supply is estimated to be $303 \mathrm{mgd}$ from surface water and 147 mgd from ground water. The supplies from the two sources overlap somewhat, because the development of the ground-water supply will reduce the surface-water supply-either by reducing ground-water discharge to the streams or by diversion of water from the stream by induced infiltration.

One approach to an estimate of the overall dependable supply would be to assume that all ground-water development would be subtracted from the surface-water supply. The dependable supply then would be the $303 \mathrm{mgd}$. A more liberal estimate of dependable supply would assume that water could be stored in surface and ground-water reservoirs to utilize more than the 7-day low flow (dependable supply) indicated in figure 18. For example, storage of surface runoff in the Kalamazoo River during the winter and early spring could double the dependable supply of the river. The amount of additional surface storage that could be developed on 


\section{WATER AVAILABILITY, KALAMAZOo COUNTY, MICHIGAN}

the Kalamazoo River, however, is small, so that utilization of surface runoff would require storage in ground-water reservoirs. The practical limits of this storage would be the amount of reduction in streamflow that would be tolerated. The total available supply (average discharge) of all streams in the county is about $680 \mathrm{mgd}$, much of which could be utilized by storage in ground-water reservoirs. In general, the longer surface water is held in the county, the greater is the potential for recharge to ground-water reservoirs.

In 1966, the withdrawal of surface water in the county was estimated to be about $58 \mathrm{mgd}$ (fig. 18). The major impact of increased withdrawal will be changes in water quality. By decreasing streamflow, less water is available for dilution of wastes. Provision for treatment of wastes, would make it possible to increase the present withdrawal of surface water.

Another important consideration in evaluation of surface-water potential is that, if the dependable supply of $303 \mathrm{mgd}$ were con-

TABLE 16.-Summary of availability and

$\begin{array}{lccc}\text { Source of water } & \text { Availability } & \begin{array}{c}\text { Present development } \\ (\text { (1966) }\end{array} & \begin{array}{c}\text { Potential } \\ \text { development }\end{array} \\ \end{array}$

Surface

Kalamazoo River at D Ave Average flow, $910 \mathrm{cfs}$; (drainage area, $1,250 \mathrm{sq}$ $\mathrm{mi})$.
Average flow, $37 \mathrm{cfs}$ low flow, $20 \mathrm{cfs}$.

Augusta (drainage area, $38.9 \mathrm{sq} \mathrm{mi)}$

Gull Creek (drainage area, Average flow, $23 \mathrm{cfs}$; $38.1 \mathrm{sq} \mathrm{mi}$ ). low flow, $5.1 \mathrm{cfs}$.

Portage Creek near Portage Average flow, $23 \mathrm{cfs}$; (drainage area, 18.6 sq low flow, $17 \mathrm{cfs}$. $\mathrm{mi})$.

Gourdneck Creek-Portage Creek (drainage area, $57.7 \mathrm{sq} \mathrm{mi}$ )

Average flow, $56 \mathrm{css}$ low flow, $26 \mathrm{cfs}$.

Portage River (drainage area, $68.2 \mathrm{sq} \mathrm{mi}$ ).
Average flow, $51 \mathrm{cfs}$ low flow, $17 \mathrm{css}$.
Flow regulated by power plant above Comstock. About 33 mgd currently withdrawn by industry and returned.

None

Lack of storage sites in the county limits development. Dependable supply limited to base flow of river.

High base flow can be augmented by storage. Present flow adequate to meet small industrial needs.

Lake level control dam With storage, up to at Cull Lake. Small about $15 \mathrm{cfs}$ may be impoundments for realized.

esthetic purposes.

Streamflow fully utilized by industry below Bryant Pond. No development above Portage.

Water diverted to West Lake from headwaters of Gourdneck Creek. Sunset Lake reservoir used to augment flow for industrial use at Vicksburg.

None.

High baseflow can be augmented by storage. Development of reser voir above Portage could have multiple use in water management.

High baseflow can be augmented by additional storage. Present flow adequate to meet current industrial demands.

Storage sites not favorable although additional storage in Indian Lake possible with lake-level control. Natural flow sufficient to meet demands of small industry. 
sumed, streams would be totally dry during low-flow periods. However, most uses of water are predominantly nonconsumptive, and about 90 percent of the water withdrawn is returned to the streams. A large increase in withdrawal of water for irrigation would greatly increase the consumptive use.

The practical limit of development or dependable supply of the major ground-water reservoirs of the county was estimated to be about $147 \mathrm{mgd}$. The estimate of $147 \mathrm{mgd}$ assumes that only about $50 \mathrm{mgd}$ would be obtained by induced infiltration of surface water. In 1966 , about $54 \mathrm{mgd}$ was being withdrawn. If pumpage increases to $70 \mathrm{mgd}$ by 1975 , there would still be a reserve of $.77 \mathrm{mgd}$. If pumpage increased to the practical limit of $147 \mathrm{mgd}$, it should not be assumed that no further development of ground water could be accomplished. The ultimate limit of ground-water development will depend on the amount of reduction in streamflow that will be tolerated. Theoretically, ground-water development could be managed

developmental factors of the water resources

\begin{tabular}{|c|c|c|c|}
\hline Quality of water & Adequacy of data & $\begin{array}{l}\text { Additional data } \\
\text { needed }\end{array}$ & $\begin{array}{l}\text { Principal problems } \\
\text { of supply and } \\
\text { development }\end{array}$ \\
\hline \multicolumn{4}{|l|}{ water } \\
\hline $\begin{array}{l}\text { Water is degraded by in- } \\
\text { dustrial-municipal dis- } \\
\text { posal below City of } \\
\text { Kalamazoo. New treat- } \\
\text { ment facilities should } \\
\text { improve quality. }\end{array}$ & $\begin{array}{l}\text { No long-term records. } \\
\text { Correlation with } \\
\text { long-term station at } \\
\text { Comstock used to } \\
\text { estimate supply. }\end{array}$ & $\begin{array}{l}\text { Occasional base-flow } \\
\text { measurements, tem- } \\
\text { peratures, and specific } \\
\text { conductance of } \\
\text { streamflow. }\end{array}$ & $\begin{array}{l}\text { Possible industrial sedi- } \\
\text { ment problems below } \\
\text { Kalamazoo. Reduction } \\
\text { of streamflow resulting } \\
\text { from inflitration into } \\
\text { ground-water } \\
\text { reservoirs. }\end{array}$ \\
\hline Good quality................ & $\begin{array}{l}\text { No long-term records. } \\
\text { Correlation used to } \\
\text { estimate supply. }\end{array}$ & $\begin{array}{l}\text { Continue record for } \\
\text { water management. } \\
\text { Detailed reservoir } \\
\text { survey. }\end{array}$ & $\begin{array}{l}\text { Possible conflict with } \\
\text { recreational (fishing) } \\
\text { use. }\end{array}$ \\
\hline $\begin{array}{l}\text { Good quality, lower in } \\
\text { mineralization than most } \\
\text { other streams in the } \\
\text { county. }\end{array}$ & .... do do........... & survey. & $\begin{array}{l}\text { New uses may conflict } \\
\text { with present recrea- } \\
\text { tional use. }\end{array}$ \\
\hline $\begin{array}{l}\text { Good quality above Por- } \\
\text { tage, slight degradation } \\
\text { below Portage and badly } \\
\text { degraded below Bryant } \\
\text { Pond. Large ground- } \\
\text { water contribution keeps } \\
\text { summer water tempera- } \\
\text { ture low. }\end{array}$ & ....do............. & $\begin{array}{l}\text { Continue record at } \\
\text { downstream station } \\
1063 \text { for water- } \\
\text { management needs. } \\
\text { Temperature and } \\
\text { specific conductance } \\
\text { of streamflow. }\end{array}$ & $\begin{array}{l}\text { New uses would con- } \\
\text { flict with present use. }\end{array}$ \\
\hline $\begin{array}{l}\text { Water of good quality } \\
\text { above Vicksburg, de- } \\
\text { graded immediately } \\
\text { below by industrial- } \\
\text { municipal waste dis- } \\
\text { posal. Quality is re- } \\
\text { covered to normal values } \\
\text { below Barton Iake. }\end{array}$ & ....do do & $\begin{array}{l}\text { Detailed reservoir sur- } \\
\text { vey, Continue records } \\
\text { on Gourdneck Creek } \\
\text { for water-manage- } \\
\text { ment needs. }\end{array}$ & $\begin{array}{l}\text { New uses would con- } \\
\text { flict with present use } \\
\text { and with future } \\
\text { ground-water devel- } \\
\text { opment. }\end{array}$ \\
\hline $\begin{array}{l}\text { Generally of good quality } \\
\text { but high in nutrients. }\end{array}$ & $\begin{array}{l}\text { Adequate for prelimi- } \\
\text { nary planning. }\end{array}$ & $\begin{array}{l}\text { Continue record for } \\
\text { water-management } \\
\text { noeds. }\end{array}$ & $\begin{array}{l}\text { Annual fluctuation of } \\
\text { Indian Lake levels } \\
\text { are largest in county. } \\
\text { (See table 12.) Addi- } \\
\text { tional storage in lake } \\
\text { would require regu- } \\
\text { latory control to } \\
\text { prevent flooding. }\end{array}$ \\
\hline
\end{tabular}


TABLE 16.-Summary of availability and

\begin{tabular}{|c|c|c|c|}
\hline Source of water & Availability & $\begin{array}{c}\text { Present development } \\
(1966)\end{array}$ & $\begin{array}{l}\text { Potential } \\
\text { development }\end{array}$ \\
\hline
\end{tabular}

Surface

West Fork Portage Creek (drainage area, $18.7 \mathrm{sq}$ $\mathrm{mi})$.

Flowerfield Creek

(drainage area, $42.6 \mathrm{sq}$ mi).

Spring Brook (drainage area, $31.1 \mathrm{sq} \mathrm{mi}$ ).

Little Portage Creek (drainage area, $27.0 \mathrm{sq}$ mi).

Average flow, $22 \mathrm{cfs}$; low flow, $5.0 \mathrm{cfs}$.

Comstock Creek (drainage area, $18.3 \mathrm{sq} \mathrm{mi}$ )

Average flow, $6.5 \mathrm{cfs}$; low flow, $3.3 \mathrm{cfs}$.

Rupert Lake Outlet

Average flow, $9.5 \mathrm{cfs}$ (drainage area, $5.27 \mathrm{sq} \mathrm{mi}$ ). low flow, $5.3 \mathrm{cfs}$.

Inland lakes:

$$
\text { Gull Lake . ........... Surface area, about }
$$

Sugarloaf Lake. Surface area, about 148 acres.

Morrow Lake........... Surface area, about 1,000 acres.

Others

About 350 other lakes and ponds ranging in size from less than an acre to several hundred.
Water used to recharge Storage would increase ground-water aquifers low flow but more by City of Kalamazoo. water would be lost by seepage to Portage Creek.

No present development. Mills formerly in operation are abandoned.

No present development.

Storage sites avallable.

Area can be considered for recreational use.

Large ground-water contribution to stream in lower reaches. With storage, modest amounts of water available for supply.

Dredged channel; aids in development of adjacent farmland and reduces bank overflow.

A maximum of about cfs is currently used by industry and returned to stream.

No present development.

Lack of storage sites limits development. Dependable supply limited to base flow of stream.

With storage, dependable flow would approach average.

Control dam at outlet. Heavy recreational use.

Control dam on outlet.

Flow is regulated by industry in Vicks. burg. Some water diverted to WestAustin Lakes.

Reservoir used to supply cooling water. ..... do

\section{Limited by present} development.

With additional storage, water would be availlable to help maintain adjacent lake levels.

Limited by present development.

Control dams at outlets Mostly for recreational of Austin, Long, and use. Modest amounts sunset I,akes. available for standby supplies.

Ground

Kalamazoo River ground- Average recharge rate water reservoir.

from precipitation, 1 mgd; from stream

infiltration, $22 \mathrm{mgd}$.

Storage, $39 \mathrm{mgd}$ for 180 days.
'Three pumping centers Rate about $39 \mathrm{mgd}$. with 19 well fields. Controlled by infilAverage daily tration from pumpage $28 \mathrm{mgd}$. Kalamazoo River.
Schoolcraft ground-water reservoir.

\author{
Average recharge rate \\ from precipitation, 18 \\ mgd. Storage rate, \\ upper aquifer, $17 \mathrm{mgd}$ \\ for 180 days; lower \\ aquifer, 13 mgd. \\ Leakage rate from \\ upper to lower \\ aquifer, $18 \mathrm{mgd}$.
}

Two pumping centers each with one well field. Average daily pumpage $0.2 \mathrm{mgd}$.

Rate about 17 mgd. Controlled by recharge from precipitation, saturated thickness, and transmissibility. Could be increased by artificial recharge. 
SUMMARY AND CONCLUSIONS

developmental factors of the water resources-Continued

\begin{tabular}{|c|c|c|c|}
\hline Quality of water & Adequacy of data & $\begin{array}{c}\text { Additional data } \\
\text { needed }\end{array}$ & $\begin{array}{l}\text { Principal problems } \\
\text { of supply and } \\
\text { development }\end{array}$ \\
\hline \multicolumn{4}{|l|}{ water-Continued } \\
\hline $\begin{array}{l}\text { Excellent quality, low in } \\
\text { dissolved solids. Low } \\
\text { ground-water contribution } \\
\text { results in higher summer } \\
\text { water temperature. }\end{array}$ & do & ......do & $\begin{array}{l}\text { New uses would con- } \\
\text { filict with present use. }\end{array}$ \\
\hline $\begin{array}{l}\text { Generally of good quality, } \\
\text { suitable for most uses. }\end{array}$ & $\begin{array}{l}\text { No long-term records. } \\
\text { Correlation used to } \\
\text { estimate supply. }\end{array}$ & .... do & Unknown. \\
\hline $\begin{array}{l}\text { Quality good, suitable for } \\
\text { most uses. }\end{array}$ & do...................... & $\ldots$........... & $\begin{array}{l}\text { Existing railroad } \\
\text { adjacent to stream } \\
\text { may prevent } \\
\text { development. }\end{array}$ \\
\hline $\begin{array}{l}\text { Suitable for most uses; } \\
\text { however, nutrients are } \\
\text { high. }\end{array}$ & $\begin{array}{l}\text { No long-term records. } \\
\text { Correlation used to } \\
\text { estimate supply. }\end{array}$ & None............... & $\begin{array}{l}\text { Dependable supply } \\
\text { limited to base fiow } \\
\text { of steam. }\end{array}$ \\
\hline $\begin{array}{l}\text { Good. Water suitable for } \\
\text { most uses. }\end{array}$ & ....................... & $\begin{array}{l}\text { Detailed reservoir } \\
\text { survey. }\end{array}$ & $\begin{array}{l}\text { New uses would conflict } \\
\text { with present use. }\end{array}$ \\
\hline do & . do do & ....do do & Unknown. \\
\hline .... do & Adequate............. & $\begin{array}{l}\text { Continue record for } \\
\text { water-management } \\
\text { needs. }\end{array}$ & $\begin{array}{l}\text { New uses would conflict } \\
\text { with present use. }\end{array}$ \\
\hline ....do. & Short-term records.... & $\begin{array}{l}\text { Detailed reservoir } \\
\text { survey. }\end{array}$ & Do. \\
\hline do & $\begin{array}{l}\text { Adequate; long-term } \\
\text { records collected at } \\
\text { Comstock. }\end{array}$ & None & None. \\
\hline nondo & $\begin{array}{l}\text { Only small sampling } \\
\text { available. }\end{array}$ & $\begin{array}{l}\text { Continue stage records } \\
\text { on West-Austin, } \\
\text { Long, Sherman, and } \\
\text { Gourdneck Lakes. }\end{array}$ & $\begin{array}{l}\text { New uses would conflict } \\
\text { with recreational use. }\end{array}$ \\
\hline
\end{tabular}

water

Degraded by infiltration of water from the Kalamazoo River and seepage from stockpiles of industrial minerals. Suitable for many industrial uses but less desirable for municlpal supplies.

Good; suitable for most uses. Iron may be objectionable and water is hard.

Good.

\section{Study of potential} recharge from stream. Observation wells to mcnitor effects of withdrawal and changes in quality.

Sparse but useful for rough estimates
Further definition of physical and hydraulic characteristics. Observation wells to monitor effects of withdrawals and change in quality.
Sealing of the sides and bottom of lakes and streams impedes recharge. Well-field sites should be determined that would balance withdrawals, infiltration, and storage. Increased withdrawals would affect water levels and chemical quality.

Well-field sites should be determined that would balance withdrawals with surface storage. Pumping centers should be located in areas with the best physical and hydraulic characteristics. New developments would conflict with surface-water use. 
TABLE 16.-Summary of availability and

\begin{tabular}{|c|c|c|c|}
\hline Source of water & Availability & Present development & $\begin{array}{l}\text { Potential } \\
\text { development }\end{array}$ \\
\hline & & & Ground \\
\hline $\begin{array}{l}\text { Kalamazoo Portage } \\
\text { ground-water reservoir. }\end{array}$ & $\begin{array}{l}\text { Average recharge rate } \\
\text { from precipitation, } 27 \\
\text { mgd. Storage, upper } \\
\text { aquifer, } 24 \mathrm{mgd} \text { for } \\
180 \text { days; lower } \\
\text { aquifer, } 14 \mathrm{mgd} \text {. } \\
\text { Leakage rate from } \\
\text { upper to lower } \\
\text { aquifer, } 27 \mathrm{mgd} \text {. }\end{array}$ & $\begin{array}{l}\text { Six pumping centers } \\
\text { with } 25 \text { well fields. } \\
\text { Average daily } \\
\text { pumpage } 21 \mathrm{mgd} .\end{array}$ & $\begin{array}{l}\text { Rate about } 24 \mathrm{mgd} \\
\text { controlled by present } \\
\text { development, satu- } \\
\text { rated thickness, and } \\
\text { transmissibility. } \\
\text { Cculd be increased } \\
\text { by artificial recharge. }\end{array}$ \\
\hline $\begin{array}{l}\text { Cooper ground-water } \\
\text { reservoir. }\end{array}$ & $\begin{array}{l}\text { A verage recharge rate } \\
\text { from precipitation, } 13 \\
\text { mgd; from stream } \\
\text { infiltration, } 7 \text { mgd. }\end{array}$ & $\begin{array}{l}\text { No pumping centers } \\
\text { or well fields. }\end{array}$ & $\begin{array}{l}\text { Rate about } 12 \text { mgd. } \\
\text { Controlled by in- } \\
\text { filtration from Kala- } \\
\text { mazoo River and } \\
\text { Spring Brook. }\end{array}$ \\
\hline $\begin{array}{l}\text { Morrow Lake ground-water } \\
\text { reservoir. }\end{array}$ & $\begin{array}{l}\text { A verage recharge rate } \\
\text { from precipitation, } 10 \\
\text { mgd; from infiltra- } \\
\text { tion, } 7 \text { mgd. }\end{array}$ & do & $\begin{array}{l}\text { Rate about } 10 \mathrm{mgd} \text {. } \\
\text { Controlled by in- } \\
\text { filtration from Morrow } \\
\text { Lake. }\end{array}$ \\
\hline $\begin{array}{l}\text { Augusta-Galesburg ground- } \\
\text { water reservoir. }\end{array}$ & $\begin{array}{l}\text { A verage recharge rate } \\
\text { from precipitation, } 14 \\
\text { mgd; from river in- } \\
\text { filtration, } 12 \mathrm{mgd} \text {. }\end{array}$ & $\begin{array}{l}\text { Two pumping centers } \\
\text { each with one well } \\
\text { field. A verage daily } \\
\text { pumpage } 0.2 \mathrm{mgd} \text {. }\end{array}$ & $\begin{array}{l}\text { Rate about } 26 \text { mgd. } \\
\text { Controlled by in- } \\
\text { filtration from Kala- } \\
\text { mazoo River, Gull } \\
\text { Creek, and Augusta } \\
\text { Creok. }\end{array}$ \\
\hline $\begin{array}{l}\text { Alamo-Oshtemo ground- } \\
\text { water reservoir. }\end{array}$ & $\begin{array}{l}\text { A verage recharge rate } \\
\text { from precipitation, } 3 \\
\text { mgd. }\end{array}$ & $\begin{array}{l}\text { No pumping centers or } \\
\text { well fields. }\end{array}$ & $\begin{array}{l}\text { Rate about } 3 \text { mgd. } \\
\text { Controlled by rate of } \\
\text { recharge from } \\
\text { precipitation. }\end{array}$ \\
\hline $\begin{array}{l}\text { Sherman Lake ground- } \\
\text { water reservoir. }\end{array}$ & $\begin{array}{l}\text { A verage recharge rate } \\
\text { from precipitation, } 7 \\
\text { mgd. }\end{array}$ & ....do do & $\begin{array}{l}\text { Rate about } 6 \text { mgd. } \\
\text { Controlled by near- } \\
\text { by Gull Lake. }\end{array}$ \\
\hline $\begin{array}{l}\text { Vicksburg ground-water } \\
\text { reservoir. }\end{array}$ & $\begin{array}{l}\text { A verage recharge rate } \\
\text { from precipitation, } 4 \\
\text { mgd. Storage, upper } \\
\text { aquifer, } 4 \text { mgd for } 180 \\
\text { days; leakage rate } \\
\text { from upper to lower } \\
\text { aquifer; } 4 \text { mgd. }\end{array}$ & $\begin{array}{l}\text { One pumping center } \\
\text { with two well fields. } \\
\text { Average daily pump- } \\
\text { age } 2 \text { mgd. }\end{array}$ & $\begin{array}{l}\text { Rate about } 6 \mathrm{mgd} \text {. Con- } \\
\text { trolled by rate of } \\
\text { recharge from pre- } \\
\text { cipitation and by in- } \\
\text { filtration from Gourd- } \\
\text { neck Creek and } \\
\text { Sunset Lake. }\end{array}$ \\
\hline $\begin{array}{l}\text { Texas ground-water } \\
\text { reservoir. }\end{array}$ & $\begin{array}{l}\text { A verage recharge rate } \\
\text { from precipitition, } 3 \\
\text { mgd. Storage, upper } \\
\text { aquifer, } 3 \text { mgd for } 180 \\
\text { days; leakage rate } \\
\text { from upper to lower } \\
\text { aquifer, } 3 \text { mgd. }\end{array}$ & $\begin{array}{l}\text { One pumping center } \\
\text { with two well fields. } \\
\text { Average daily pump- } \\
\text { age } 2.5 \text { mgd. Woter } \\
\text { is recirculated. }\end{array}$ & $\begin{array}{l}\text { Rate about } 3 \text { mgd. } \\
\text { Controlled by level of } \\
\text { lakes used for recrea- } \\
\text { tion. Could maintain } \\
\text { water level by infl- } \\
\text { tration from West } \\
\text { Fork Portage Creek. }\end{array}$ \\
\hline $\begin{array}{l}\text { Isolated well field (north- } \\
\text { west extension of Kala- } \\
\text { mazoo Portage ground- } \\
\text { water reservoir.) }\end{array}$ & Not determined......... & $\begin{array}{l}\text { One well field. Average } \\
\text { daily pumpage } 0.3 \\
\text { mgd. }\end{array}$ & $\begin{array}{l}\text { Rate small. Controlled } \\
\text { by physical and hy- } \\
\text { draulic characteristics } \\
\text { of lower aquifer. No } \\
\text { upper aquifer. }\end{array}$ \\
\hline
\end{tabular}

so that little or no streamflow would leave the county. Practically, diversion of surface runoff during winter and early spring to recharge aquifers could increase the potential ground-water development to as much as $300 \mathrm{mgd}$-about twice as much as estimated in the reservoir analyses. Greater use of artificial ponds and basins to 
developmental factors of the water resources-Continued

\begin{tabular}{|c|c|c|c|}
\hline Quality of water & Adequacy of data & $\begin{array}{c}\text { Additional data } \\
\text { needed }\end{array}$ & $\begin{array}{l}\text { Principal problems } \\
\text { of supply and } \\
\text { development }\end{array}$ \\
\hline \multicolumn{4}{|l|}{ Water-Continued, } \\
\hline $\begin{array}{l}\text { Good throughout most of } \\
\text { area but artifictal } \\
\text { recharge of poor quality } \\
\text { water has degraded a } \\
\text { small area. }\end{array}$ & Excellent............. & $\begin{array}{l}\text { Observation wells to } \\
\text { monitor effects of } \\
\text { withdrawal and } \\
\text { changes in quality. }\end{array}$ & $\begin{array}{l}\text { Increased withdrawals } \\
\text { would affect water } \\
\text { levels and chemical } \\
\text { quality. Further inves- } \\
\text { tigation of stream } \\
\text { infiltration and } \\
\text { artificial recharge is } \\
\text { needed. }\end{array}$ \\
\hline $\begin{array}{l}\text { Presently good. Quality } \\
\text { may deteriorate if poor } \\
\text { quality water is } \\
\text { infiltrated. }\end{array}$ & Inadequate.....- & $\begin{array}{l}\text { Definition of physical } \\
\text { and hydraulic char- } \\
\text { acteristics of reservior. } \\
\text { Observation wells to } \\
\text { monitor effects of } \\
\text { withdrawal and } \\
\text { quality. }\end{array}$ & $\begin{array}{l}\text { Sealing of the sides and } \\
\text { bottom of streams } \\
\text { impedes recharge. } \\
\text { Well-field sites should } \\
\text { be determined that } \\
\text { would balance with- } \\
\text { dra "als, inflitration, } \\
\text { and storage. }\end{array}$ \\
\hline $\begin{array}{l}\text { Good. Suitable for most } \\
\text { uses. Iron may be ob- } \\
\text { jectionable and water } \\
\text { is hard. }\end{array}$ & ......do............. & $\begin{array}{l}\text { Further definition of } \\
\text { physical and } \\
\text { hydraulic character- } \\
\text { istics and potential } \\
\text { recharge from } \\
\text { streams. Observation } \\
\text { wells to monitor ef- } \\
\text { fects of withdrawal } \\
\text { and change in } \\
\text { quality. }\end{array}$ & Do. \\
\hline ....do. & Insdequate.... & $\begin{array}{l}\text { Definition of physical } \\
\text { hydraulic character- } \\
\text { istics. Observation } \\
\text { wells to monitor of- } \\
\text { fects of withdrawal } \\
\text { and quality. }\end{array}$ & $\begin{array}{l}\text { Pumping centers should } \\
\text { be located in areas } \\
\text { with the best physical } \\
\text { and hydraulic char- } \\
\text { acteristics. Yield } \\
\text { could be incresed } \\
\text { by artiflcial recharge. }\end{array}$ \\
\hline 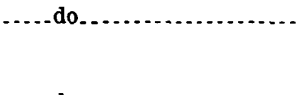 & (n) do & do & $\begin{array}{l}\text { Investigation of stream } \\
\text { infiltration and } \\
\text { artificial recharge } \\
\text { is needed. }\end{array}$ \\
\hline -....do.... & Fair . - & $\begin{array}{l}\text { Further definition of } \\
\text { physical and hydraulic } \\
\text { characteristics. Ob- } \\
\text { servation wells to } \\
\text { monitor effects of } \\
\text { withdrawal and } \\
\text { change in quality. }\end{array}$ & $\begin{array}{l}\text { Sealing of sides and } \\
\text { bottom of streams and } \\
\text { lakes impedes re- } \\
\text { charge. Well-field sites } \\
\text { should be determined } \\
\text { that would balance } \\
\text { withdrawals, infiltra- } \\
\text { tion, and storage. }\end{array}$ \\
\hline - do & Good............. & $\begin{array}{l}\text { Observation wells to } \\
\text { monitor effects of } \\
\text { withdrawal and } \\
\text { change in quality. }\end{array}$ & $\begin{array}{l}\text { The best rate of with. } \\
\text { drawal should be } \\
\text { determined that } \\
\text { would balance } \\
\text { storage. }\end{array}$ \\
\hline
\end{tabular}


The chemical quality of water in the streams of Kalamazoo County is generally good; however, the Kalamazoo River downstream from Kalamazoo is affected by industrial wastes. Other significant streams affected are the lower reach of Portage Creek at Kalamazoo, Gourdneck-Portage Creek downstream from Vicksburg, and ${ }^{\circ}$ Allen Creek near Kalamazoo. Ground water is of good chemical quality, although it is hard and locally has objectionable arnounts of iron. To maintain the quality of surface and ground water, management must be alert to the changes that may accompany future development. Inducing poor quality water into aquifers can eliminate them as sources of usable water, and in some instances may reduce the transmissibility of the aquifers. Accidental release of toxic materials that percolate to ground-water reservoirs can destroy the reservoir for a long timeperhaps for all time. Water management should consider preserving the quality as well as the quantity of the water resources.

\section{SELECTED REFERENCES}

American Public Health Association, American Water Works Association, and Water Pollution Control Federation, 1965, Standard methods for the examination of water, sewage and industrial wastes [12th ed.] : New York, Am. Public Health Assoc. Inc., 176 p.

Bentall, Ray, compiler, 1963a, Methods of determining permeability, transmissibility, and drawdown: U.S. Geol. Survey Water-Supply Paper 1536-I, p. 243-341.

$1963 \mathrm{~b}$, Methods of collecting and interpreting ground-water data: U.S. Geol. Survey Water-Supply Paper 1544-H, 97 p.

1963c, Shortcuts and special problems in aquifer tests: U.S. Geol. Survey Water-Supply Paper 1545-C, 117 p.

Bolton, N. S., 1963, Analysis of data from non-equilibrium pumping tests allowing for delayed yield from storage : Inst. Civil Engineers Proc., v. 26, no. 6693, p. 469-482.

Comly, H. J., 1945, Cyanosis in infants caused by nitrates in well water: Am. Med. Assoc. Jour., v. 129, p. 112-116.

Csallany, S. C., 1966, Graphical method for determination of coefficient of transmissibility : Jour. Am. Water Works Assoc., v. 58, no. 5, p. 628-634.

Deutsch, Morris, Vanlier, K. E., and Giroux, P. R., 1960, Ground-water hydrology and glacial geology of the Kalamazoo area, Michigan: Michigan Geol. Survey Prog. Rept. 23, 122 p., 21 figs.

Ferris, J. G., Knowles, D. B., Brown, R. H., and Stallman, R. W., 1962, Theory of aquifer tests: U.S. Geol. Survey Water-Supply Paper 1536-E, p. 69-174.

Giroux, P. R., Stoimenoff, L. E., Nowlin, J. O., and Skinner, E. L., 1966, Water resources of Branch County, Michigan: Michigan Geol. Survey Water Inv. 6, $158 \mathrm{p}$.

Graf, D. L., Friedman, I., and Meents, W. F., 1965, The origin of saline formation waters-[Part] 2, Isotopic fractionation by shale micropore systems: Illinois Geol. Survey Circ. 393, 32 p.

Hantush, M. S., and Jacob, C. E., 1954, Plane potential flow of ground water with linear leakage : Am. Geophys. Union Trans., v. 35, no. 6, p. 917-936. 
Heath, R. C., 1964, "Lazy" thermometers and their use in measuring groundwater temperatures in Short papers in geology and hydrology: U.S. Geol. Survey Prof. Paper 475-D, p. D216-D218.

Humphreys, C. R., and Green, R. F., 1962, Michigan lake inventory : Michigan State Univ., Dept. Resource Devel. Bull. 39, p. 39A-39C.

Jacob, C. E., 1944, Notes on determining permeability by pumping tests under water-table conditions : U.S. Geol. Survey open-file rept.

Jenkins, C. T., 1963, Graphical multiple-regression analysis of aquifer tests in Short papers in geology and hydrology: U.S. Geol. Survey Prof. Paper 475-C, p. C198-C201.

Joint Committee on Water Resources Planning 1966, Michigan laws relating to water : Legislative Service Bur., Lanısing, Mich., $415 \mathrm{p}$.

Kohler, M. A., Nordenson, T. J., and Baker, D. R., 1959, Evaporation maps for the United States: U.S. Weather Bureau Tech. Paper 37, 13 p., 5 pl.

Maier, F. J., 1950, Fluoridation of public water supplies: Am. Water Works Assoc. Jour., v. 42, pt. 1, p. 1120-1132.

Michigan Department of Health, 1961, Data on public water supplies in Michigan : Michigan Dept. Health Eng. Bull. 4, 57 p.

Michigan Water Resources Commission, 1968, Water quality standards for Michigan Intrastate Waters : Lansing, Mich., Mich. Water Resources Comm., $39 \mathrm{p}$.

Olmsted, F. H., and Hely, A. G., 1962, Relation between ground water and surface water in Brandywine Creek Basin, Pennsylvania: U.S. Geol. Survey Prof. Paper 417-A, $21 \mathrm{p}$.

Rainwater, F. H., and Thatcher, L. L., 1960, Methods for collection and analysis of water samples : U.S. Geol. Survey Water-Supply Paper 1454, $301 \mathrm{p}$.

Rasmussen, W. C., and Andreasen, G. E., 1959, Hydrologic budget of the Beaverdam Creek basin, Maryland: U.S. Geol. Survey Water-Supply Paper 1472, $106 \mathrm{p}$.

Reed, J. E., Deutsch, Morris, and Wiitala, S. W., 1966, Induced recharge of an artesian glacial-drift aquifer at Kalamazoo, Michigan: U.S. Geol. Survey Water-Supply Piaper 1594-D, 62 p.

Schicht, R. J., and Walton, W. C., 1961, Hydrologic budgets for three small watersheds in Illinois : Illinois State Water Survey Rept. Inv. 40, 40 p.

Swenson, H. A., and Baldwin, H. L., 1965, A primer on water quality: Washington, U.S. Govt. Printing Office, 27 p.

Theis, C. V., 1941. The effect of a well on the flow of a nearby stream: Am. Geophys. Union Trans., v. 22, pt. 3, p. 734-738.

U.S. Public Health Service, 1962, Drinking water standards : U.S. Public Health Service Pub. 956, $61 \mathrm{p}$.

U.S. Salinity Laboratory Staff, 1954, Diagnosis and improvement of saline and alkali soils: U.S. Dept. Agriculture, Agriculture Handb. 60, 160 p.

Veihmeyer, F. J., 1964, Evapotranspiration, Section II in V. T. Chow, ed., Handbook of Applied Hydrology: New York. McGraw-Hill Book Co., 38 p.

Walton, W. C., 1962, Selected analytical methods for well and aquifer evaluation : Illinois State Water Survey Bull. 49, 81 p.

Weeks, E. P., 1964, Field methods for determining vertical permeability and aquifer'anisotropy : U.S. Geol. Survey Prof. Paper 501-D, p. D193-D198.

Wiitala, S. W., 1965, Magnitude and frequency of floods in the United States, Part 4, St. Lawrence River Basin: U.S. Geol. Survey Water-Supply Paper, $1677,357 \mathrm{p}$. 



\section{INDEX}

[Italic page numbers indicate major references]

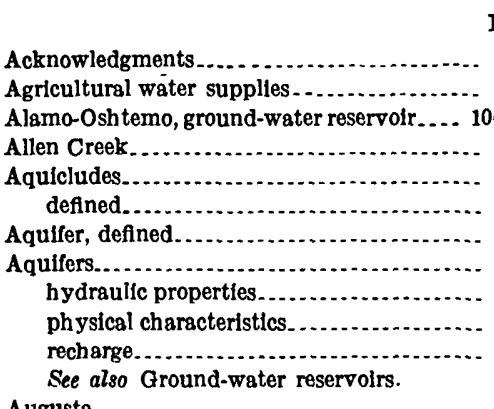

Avgusta. ................................ 106

Augusta Creek...................... 6, 20,44,45,70

Augusta Creek near Augusta................ 33

Augusta-Galesburg, ground-water reservoir. 104, 106

Austin Lake.

$2,7,12,65$ chemical quallty

Austin Lake complex water budget

Barton Lake

chemical quality

Basal aquiclude.

Bass Lake.

Bear Creek................................. 19

Bedrock, consolidated rocks.................. 7

Blbliography .............................. 124

Brandywine Creek basin, Pennsylvania ...... 13

Chemical analyses.

Chemical quality $31,46,70,87,101,107,112$

Climatic records

Coefficient of transmissibility, defined.

Comstock Creek near Kalamazoo.

Confining layer.

Consolldated bedrock.

Cooper ground-water reservolr

Crooked Lake............................ 3.63 chemical quality...................... $\quad 75$

Damsites, potential

Darcy's equation

70
$13,60,89$

Deposits, unconsolidated.

Dewatering equation $\quad 79$

Diversion canal.............................. 59

Domestic water supplles.................... 26

Draft-storage frequency analyses.............. 41

Drought.................................... 2,67

Eagle Lake................................. 3,63 chemical quality.
Fvaporation

Evapotranspiratio

Field conditions, comparison with model conditions

Flood-frequency curves....................... 44

Floods...................................... 44

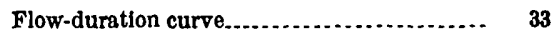

Flowerfield Creek .......................... 6,20

Frequency-mass curves...................... 40

Gaging, partlal-record stations................ 37

Galesburg................................ 106

Glacial materials, transmissibllity............ 18

Gourdneck Creek................... 6, 18, 44, 49, 70

Gourdneck Lake ............................ 7,65 chemical quality......................... 73

Ground water............................... 28

augmentation ............................ 63

budgets. ................................. 12

chemical analyses...................... 21

divides.................................. 18

Idealized reservoirs. ................... 81

movement............................... 18,78

natural discharge......................... 18

pumpage............................. 115

sultability for municipal use.............. 28

temperature variat!on with depth........ 21

withdrawals............................. 114

Ground-water reservolr, Alamo-Oshtemo..... 107

Augusta Galesburg................... 106

Cooper.............................. 104

Kalamazoo-Portage

Kalamazoo River........................... 80

Morrow Lake.......................... 105

Schoolcraft.......... 87

Sherman Lake. ......................... 108

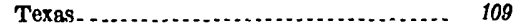

Vicksbung................................ 108

Ground-water reservoirs..................

defined..................................... 11

development.......................... 85

mathematical model...................... $\quad 79$

potential development, summary........ 111

potential yleld......................... $\quad 77$

yield, infiltration...................... 78

Ground-water system..................... 8

Gull Creek.................................. 6, 106

Gull Lake.................................... 7

chemical quality........................ 75 


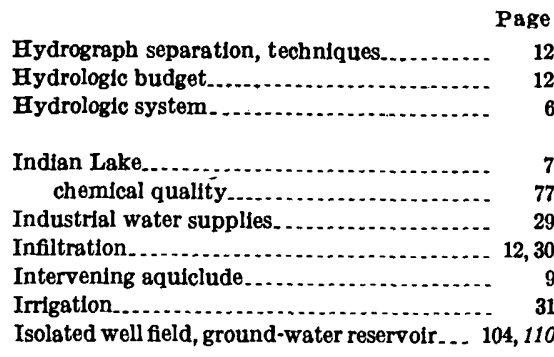

Kalamazoo moraine.

Kalamazoo-Portage ground-water reservoir...

Kalamazoo River.

Kalamazoo River at Comstock. . . . . . . . . . 12, 32

Kalamaz00 River basin........................ 12

Kalamazoo State Hospital, climatic records .. $\quad 5,29$

Lake, Austin.

Barton.

Crooked

Eagle.

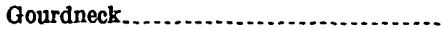

Gull.

Indian

Long.

Sherman.

Sugarloaf.

Lakes....

heating effects...................... 30

levels............... 3

Legal considerations.............

Lithologic sections........................... 9

Little Portage Creek......................... 6,19

Little Portage Creek near Fulton............. 33

Long Lake. . . . . . . . . . . . . . . . .

chemical quality....................... 73

Low-flow Index............................... 34, 41

Lower squifer. . ...................... 65, 104, 110

Manmade storage reservoirs.................. 66

Marshes. . . . . . . .

Mathematical models, ground-water reservoirs.

Morrow Lake.

ground-water reservolr................ 104, 105

Multiple regression analysis.

Nonreservolr areas.

Objectives. See Purpose and scope of study.

Permeability, field coefflcient defined.

Pit Iakes.

Portage Creek.

Portage Creek, Kalamazoo basin.

Portage Creek near Kalamazoo..............

Portage Creek near Portage........ . 20, 34, 44, 49, 71

Portage River ............................ 6, 19, 20

Portage River near Vicksburg . . .............. 12, 33

Potential development, ground-water reservolrs, summary.

Precipitation.

Public water supplies.
Pumplng centers, development............. . . 85,92

Purpose and scope of study ................ 3,77

Quallty..................................... 21 chemical............ 31, 46, 71, 87, 90, 101, 107, 112 chemical analyses..................... 21, 50 relation to use ............................ 26

Recharge................ 12, 77,93

artlficial ................ 97

Kalamazoo River...................... 81

Kalamazoo River basin................ 15

St. Joseph River basin. ................. 13

Recurrence Interval, low flow................

Reservoir sites, potential.................. 70

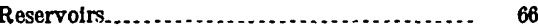

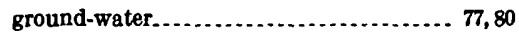

Ideallzed............................. 88,92

Schoolcraft. ....................... 87

RIver water............................. 28

cooling use........................... 30

Rupert Lake. . . . . . . . .

outlet.................................... 44

St. Joseph RIver basin . . ................... 6,12

Baturated thickness, aquifers............... 9

upper aquifer....................... 19

Schoolcraft ground-water reservolr............ 87

future development...................... $\quad 89$

water quality............................ 91

Seepage $\ldots \ldots \ldots \ldots \ldots . .18,33,87$

Seepage run. . .............................. 18

Semiconfining layer..................... 9

Seven Mile Creek............................... 70

Sherman Lake, chemical quality. ........... $\quad 76$

ground-water reservolr.............. 104, 106, 108

Snowfall...................................... 6

Specific capacity, defined.................. 9

Spring Brook.............................. 6,20

Storage analysis........................... 40

Storage coefficient, defined................... 11

Storage requirements...................... 40,41

Streamflow, augumentation................ 54 augumentation, ground water to Crooked and Eagle Lakes.

Sugarloaf Lake to West-Austin Lakes. base-flow measurements................... characteristics......................... 36 data.

duration curves.......................... 33 low-flow frequency.................... 34 storage supplement................... 40

withdrawals.............................. 48

Streams.

Subsurface inflow...........................

Subsurface outflow ....................... 69

Subsurface underflow, computation.......... 13

Sugarloaf Lake. ........................ 65

chemical quality..................... 73

Summary and conclusions................. 117

Surface water, chemical analyses............. 28

Surface-water system... . . . . . . . . . . . . . . . 6 


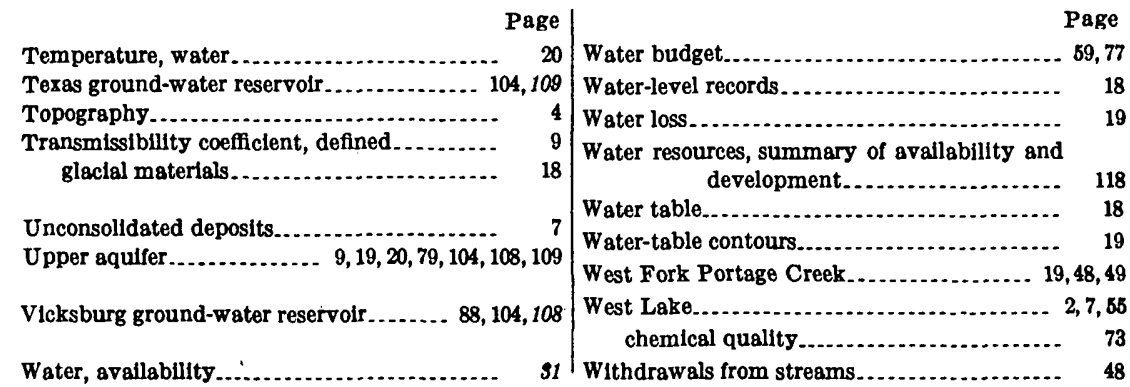

